\title{
DESIGN AND COST PERFORMANCE OF WDM PONs FOR MULTI-WAVELENGTH USERS
}

\author{
Dissertation \\ zur Erlangung des akademischen Grades \\ Doktoringenieur (Dr.-Ing.)
}

vorgelegt der Fakultät für Elektrotechnik und Informationstechnik der Technischen Universität Ilmenau

von Dipl.-Ing. (FH) Jürgen Schussmann geboren am 01.09.1970 in Klagenfurt

vorgelegt am 28. April 2008

Gutachter: 1. Univ.-Prof. Dr. rer. nat. habil. Jochen Seitz

2. Dr.-Ing. Werner Horn

3. FH-Prof. Dr. Dipl.-Ing. Thomas Schirl

Verteidigung am 15. September 2008

Verf.-Nr.: EI 


\section{Table of Contents}

Preface (1)

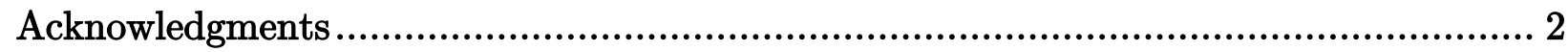

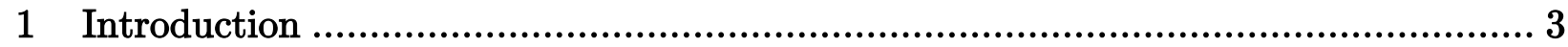

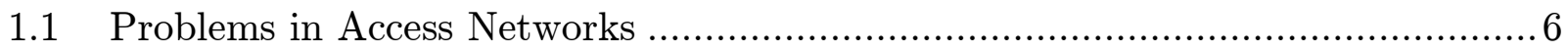

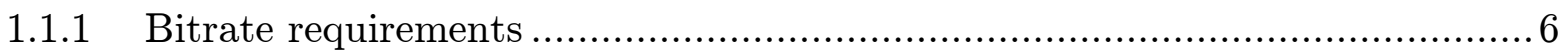

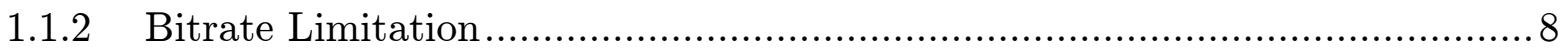

1.1.3 Current Technologies in Access Networks................................................. 11

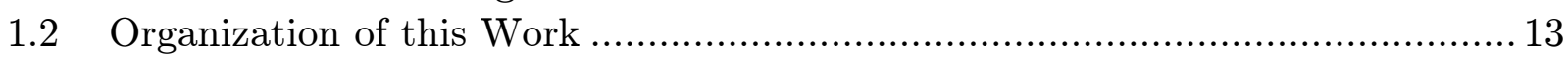

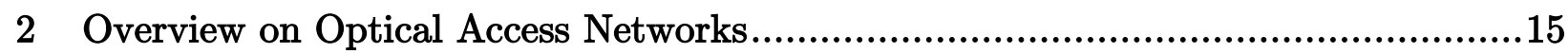

2.1 Classification of Optical Access Networks................................................... 15

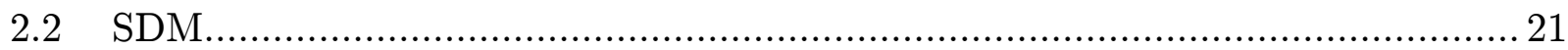

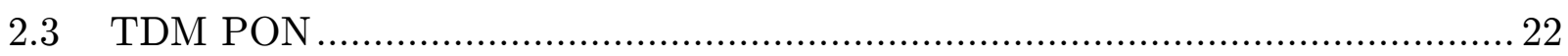

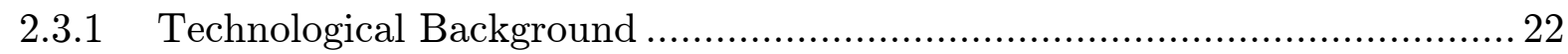

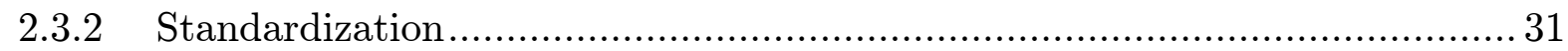

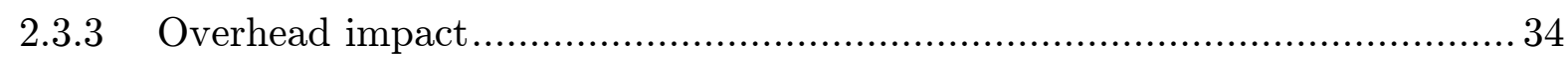

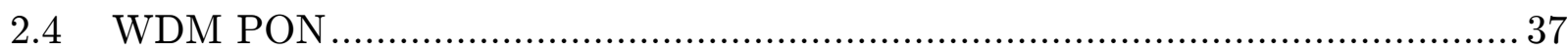

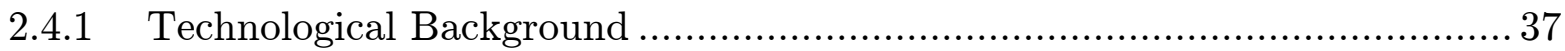

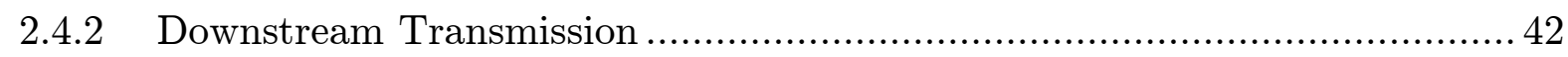

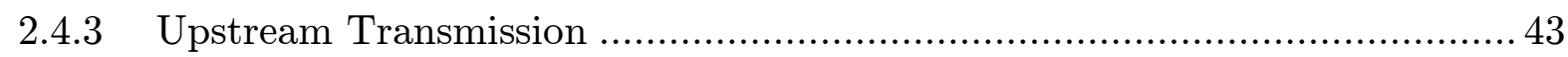

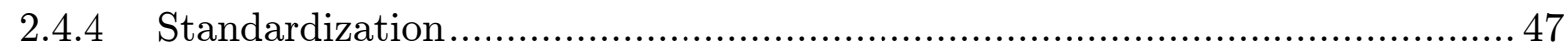

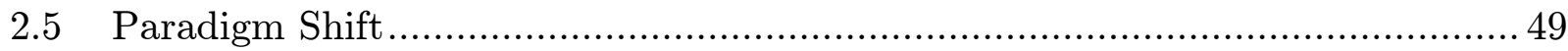

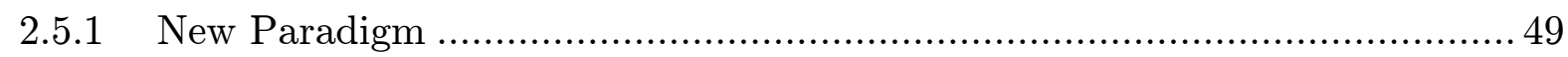

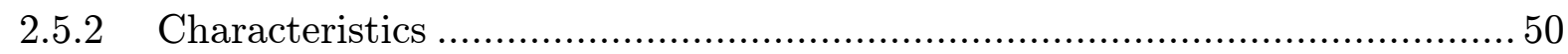

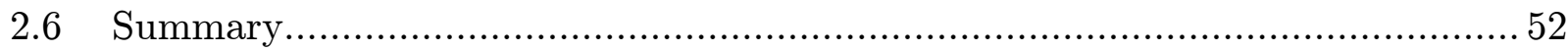

$3 \quad$ Single-Stage Networks.....................................................................................54

$3.1 \quad$ Wavelength reuse and space demultiplexing ...................................................5 54

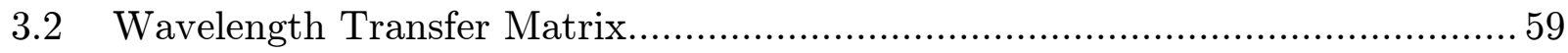

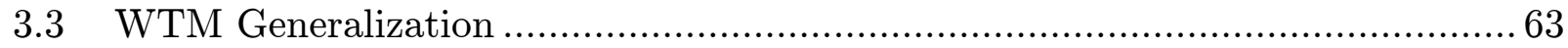

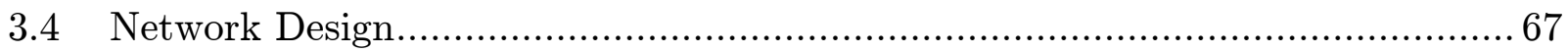

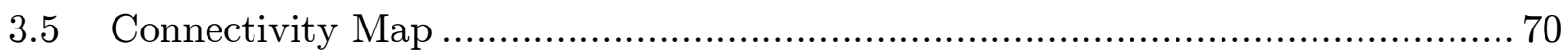

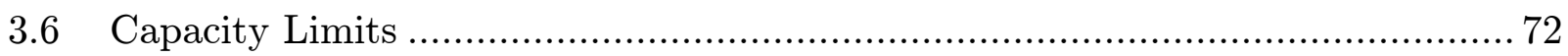

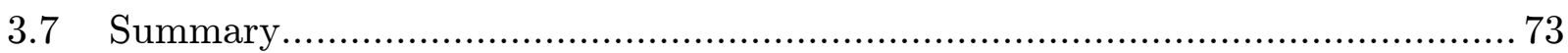

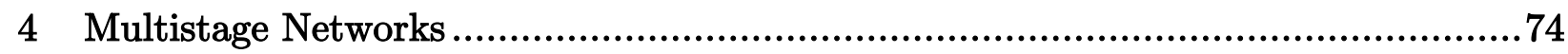

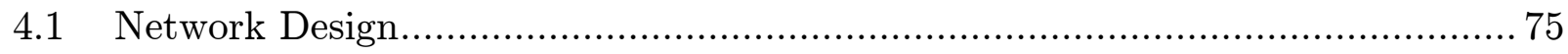

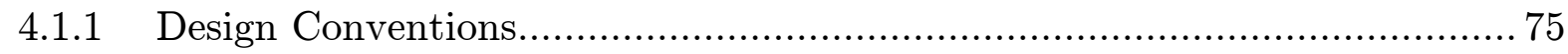

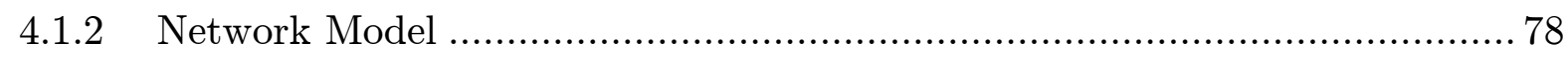


4.1.3 Constraints of the Design Conventions .................................................. 78

4.1.4 Common Design Parameters .......................................................... 79

4.1 .5 IC WDM PON ........................................................................... 81

4.1 .6 DC WDM PON ......................................................................... 85

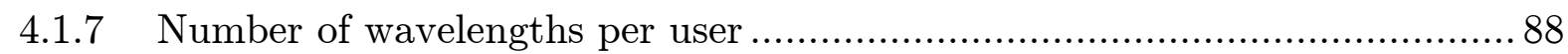

4.1 .8 IC versus DC WDM PONs ............................................................ 88

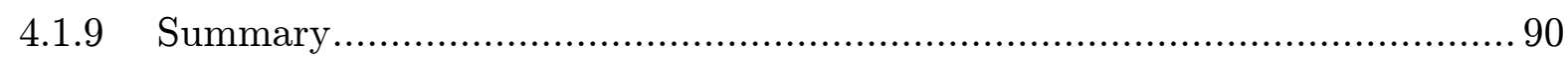

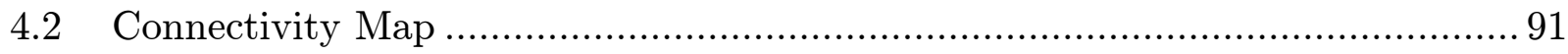

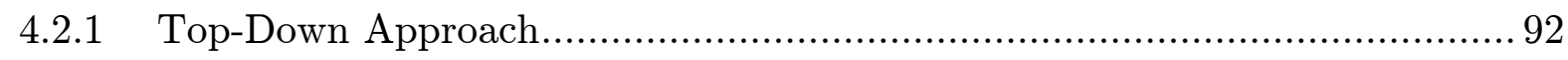

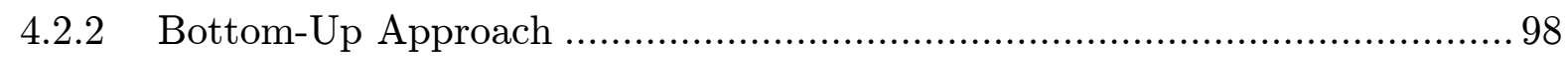

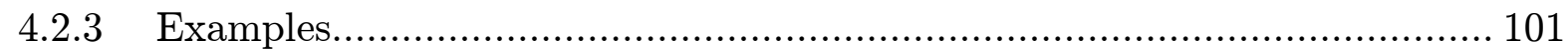

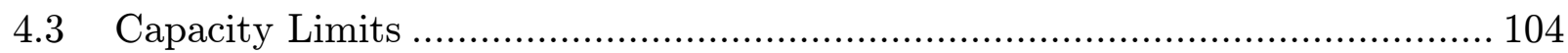

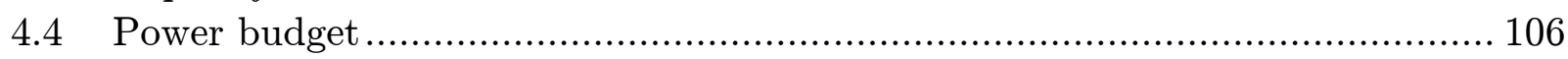

5 Techno- Economic Evaluations ..................................................... 108

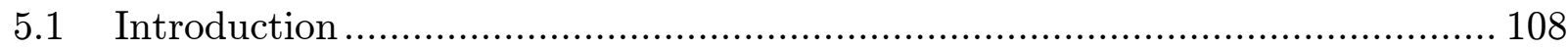

5.2 Generation of Network Architectures.................................................. 109

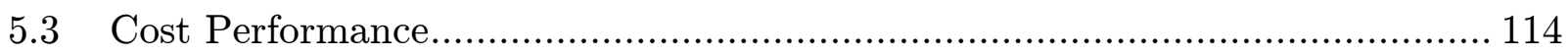

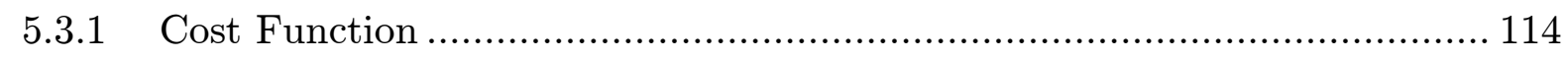

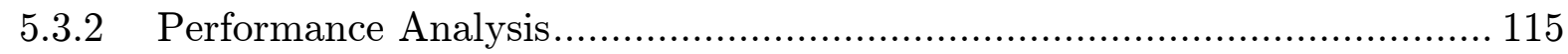

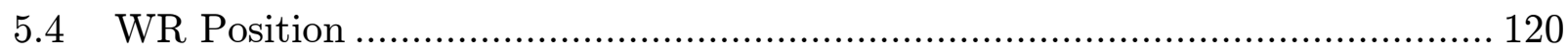

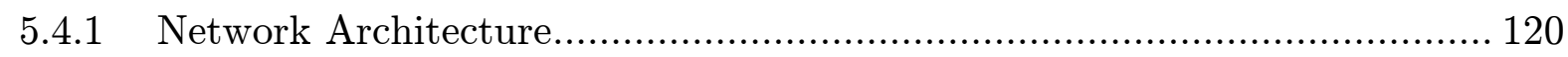

5.4 .2 Performance Analysis...................................................................... 123

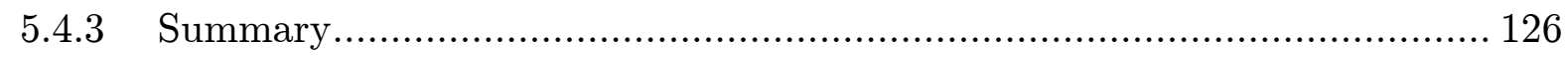

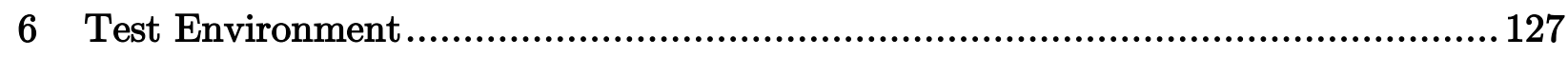

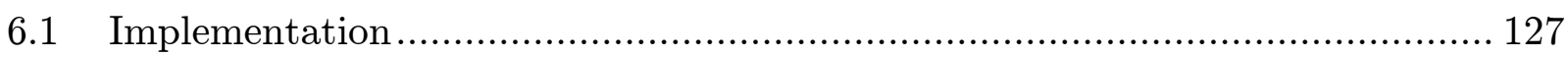

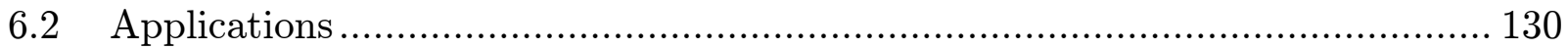

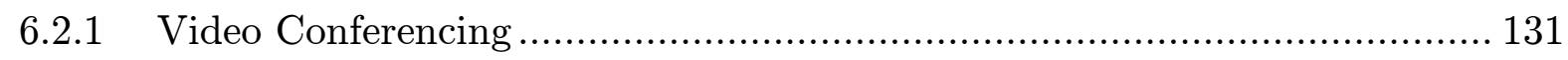

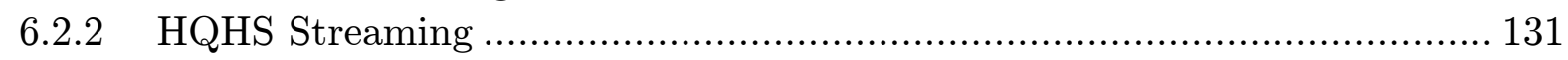

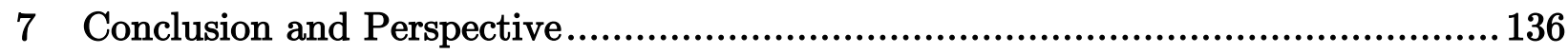

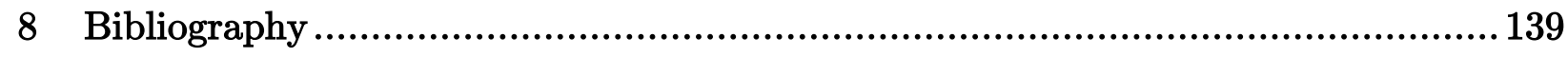

9 Appendix A List of Abbreviations .................................................... 155

10 Appendix B List of used Symbols .................................................... 161

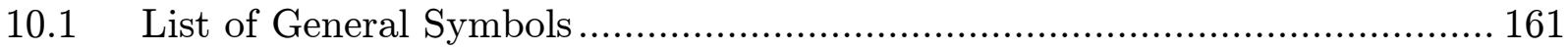

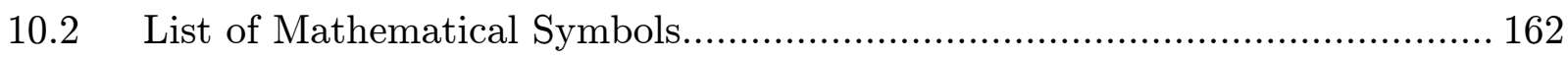

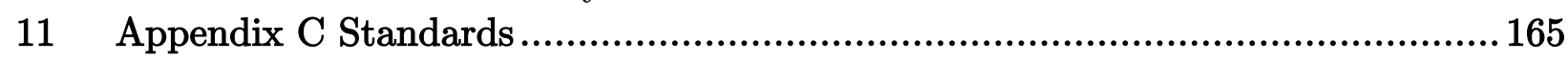

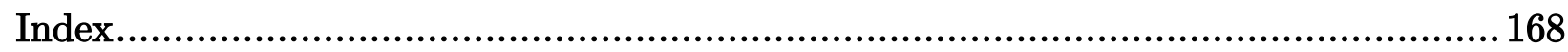




\section{Preface}

Due to the incomparable popularity of the World Wide Web, the already enormous and still rocketing bandwidth demand may only be satisfied by optical networks, particularly by using the Wavelength Division Multiplexing (WDM) technology. To obtain WDM signals with high bit rates, multiple channels operating at a lower transmission speed are supported on a single optical fiber. This technology is currently applied exclusively in core and distributed networks.

The subject of this thesis will be the analysis of WDM-based passive optical networks (PON) in the access domain. The paradigm shift, one wavelength per service, leads to the introduction of new aspects in the design of future WDM PON access networks.

New cutting-edge architectures offer each service its own wavelength across the whole access network. Only architectures which guarantee a multi-provider solution are analyzed to provide substantial value enhancement for the customer.

This work deals with single and multistage WDM PONs. New methods are introduced to design and calculate these future optical access networks which fulfill the established paradigm shift. Required design parameters are identified and new methods are introduced to calculate all necessary parameters to design such networks. These new design conventions can be used to fully calculate each WDM PON relating to the functional requirements.

In techno-economic evaluations, new network architectures with the highest potential concerning economic considerations are identified. Thereby, technological feasibility is a basic condition. The purpose is to find out which combinations of design parameters are significant and in particular minimize costs. A tool was developed to fully calculate the costs and a connectivity map of new architectures. New down-to-earth architectures need to be analyzed and evaluated.

The area-wide deployment of WDM PONs introduces more simply structured systems that are cheaper in the short or medium term. Thereby, an introduction to this new technology will speed up market introduction as well as market acceptance. This will help WDM PON access networks become reality. 


\section{Acknowledgments}

This work is the result of my Ph.D. studies performed as Ph.D. student at the Institute of Communication Networks of the Faculty of Electrical Engineering and Information Technology at the Technical University in Ilmenau. The whole work was performed at the Institute of Telematics/Network Engineering at the Carinthia University of Applied Sciences in Austria.

I owe special thanks to my adviser, Univ.-Prof. Dr. rer.nat. Jochen Seitz, for his continuous encouragement, guidance and motivation in making this dissertation possible. Thanks especially go to Dr. Werner Horn, who read through my work and supported me with her expertise.

I am highly grateful for the exciting and profitable discussions and the countless refreshing sports activities initiated by Prof. (FH) Dipl.-Ing. Dr. rer.nat. Thomas Schirl. His ideas and tremendous support have a major influence on this thesis. He spent a lot of time helping me.

I also want to thank all the other colleagues at the Institute of Telematics/Network Engineering at the Carinthia University of Applied Sciences that have fed this work with valuable support, comments and exciting discussions. Thanks a lot to Univ.-Doz. Dipl.-Ing. Dr. techn. Herbert Stögner for the fascinating discussions which opened up my horizon. Many thanks to Sabine Hofstätter and Marvin Hoffland for proofreading this work and for the stimulating discussions.

Last but not least, I am obliged to my whole family for their infinite patience and the implicit faith in my capabilities; my gratitude is boundless and cannot be expressed in words. A lot of thanks to my parents, Ernst and Inge, and my sister Sabine. Without their support, encouragement and help I would not have been able to complete this thesis. I'm especially grateful to my girlfriend Gabriele for helping me keep my life in a proper perspective and balance. Gabriele, thank you for your unyielding devotion, dedication and love. I am proud that I can mention my wonderful son Marcel. This work is devoted to my grandmother Maria.

Last, I want to encourage those people who are considering a Ph.D. It means a lot of work but it is an experience well worth it. 


\section{Introduction}

$\mathrm{I}$

$\mathrm{n}$ principle, the rapid growth and ever increasing bandwidth due to the Internet explosion justifies the need for high-speed access networks. In particular, wavelength division multiplexing (WDM) technology [Mukherjee97, Ramaswami02, Banerjee05, Lin06] is commonly viewed as the preferred choice in building such future high-speed access networks. In WDM networks, multiple wavelength channels operating at electronic transmission rates are supported in parallel to fully utilize the enormous potential bandwidth of a single optical fiber. Optical networks relying on optical fiber transmission are characterized by extremely low bit error rates (BER) on the order of $10^{-15}$. The potential bandwidth of a single optical fiber lies in the Tbit/s range within the low-loss transmission window around $1300 \mathrm{~nm}$ and $1550 \mathrm{~nm}$. In consequence, the transmission speed limitation is caused by the electronics at the end nodes.

Photonic (photon and electronics) networks are also known as optical networks or alloptical networks. Within these networks the information remains in the optical domain during its transfer from the source node to the destination node, thereby ensuring full transparency to data and modulation formats. Therefore, the conversation between the optical and the electrical domain, commonly known as the electronic bottleneck, is avoided. In principle, photonic networks can be realized by using optical circuit switching or optical packet switching. In optical circuit switching networks, a wavelength or lightpath is dedicated to a certain connection for the duration of the communication whereas in optical packet switching networks, the data transmission is performed on a packet-by-packet basis.

Although the resource efficiency is relatively low in optical circuit switching networks, it is a relatively simple and cost effective solution without quality of service (QoS) problems within a wavelength or lightpath. Due to these reasons, we only consider optical circuit switched networks throughout this work.

In order to fully exploit the tremendous transmission bandwidth of an optical fiber, different multiplexing techniques such as WDM, optical time division multiplexing (OTDM) [Ramaswami02] and optical code division multiplexing (OCDM) [Kitayama98] may be applied in optical networks. WDM divides the enormous potential bandwidth of a single optical fiber into different wavelength channels (these days supporting several hundred wavelengths). Each channel operates in parallel at transmission rates processable by electronics. In OTDM, ultra short pulses (in the lower ps-range) are used to generate extremely high data rates, while OCDM relies on the interference of distinct optical codes. WDM networks are relative easy to implement compared to the other schemes, since all components generally need to operate only at electronic speed. 
Therefore, lots of WDM components and systems are commercially available and deployed today [Schussmann08b]. As a result, we solely refer to WDM networks throughout this work.

Nowadays, the WDM technique continues its success story in wide area networks (WAN) and is rapidly emerging as viable and cost effective solution for metropolitan area networks (MAN). Thus, the need for the development of novel high-speed architectures in access networks intensively arises. This work focuses on optical access networks based on the WDM technique.

Another motivation for high-speed access networks have been the ever rising bitrate requirements far a long time. Several high bitrate access solutions are technically feasible. However, their commercial launch depends on many parameters like the complex socio-economic process of application pull, the low cost production capabilities, competition, the rollout of infrastructure and a further technology push. The access bitrate, have led to an exponential increase (Figure 1.1). When we go from the early voiceband modems (V.xx) and integrated services data networks (ISDN) [Kanbach99] to digital subscriber lines (DSL), an extrapolation shows that a bandwidth between $0.1 \mathrm{Gbit} / \mathrm{s}$ and $10 \mathrm{Gbit} / \mathrm{s}$ per user is expected to become generally available after 2010 [Söderlund04].

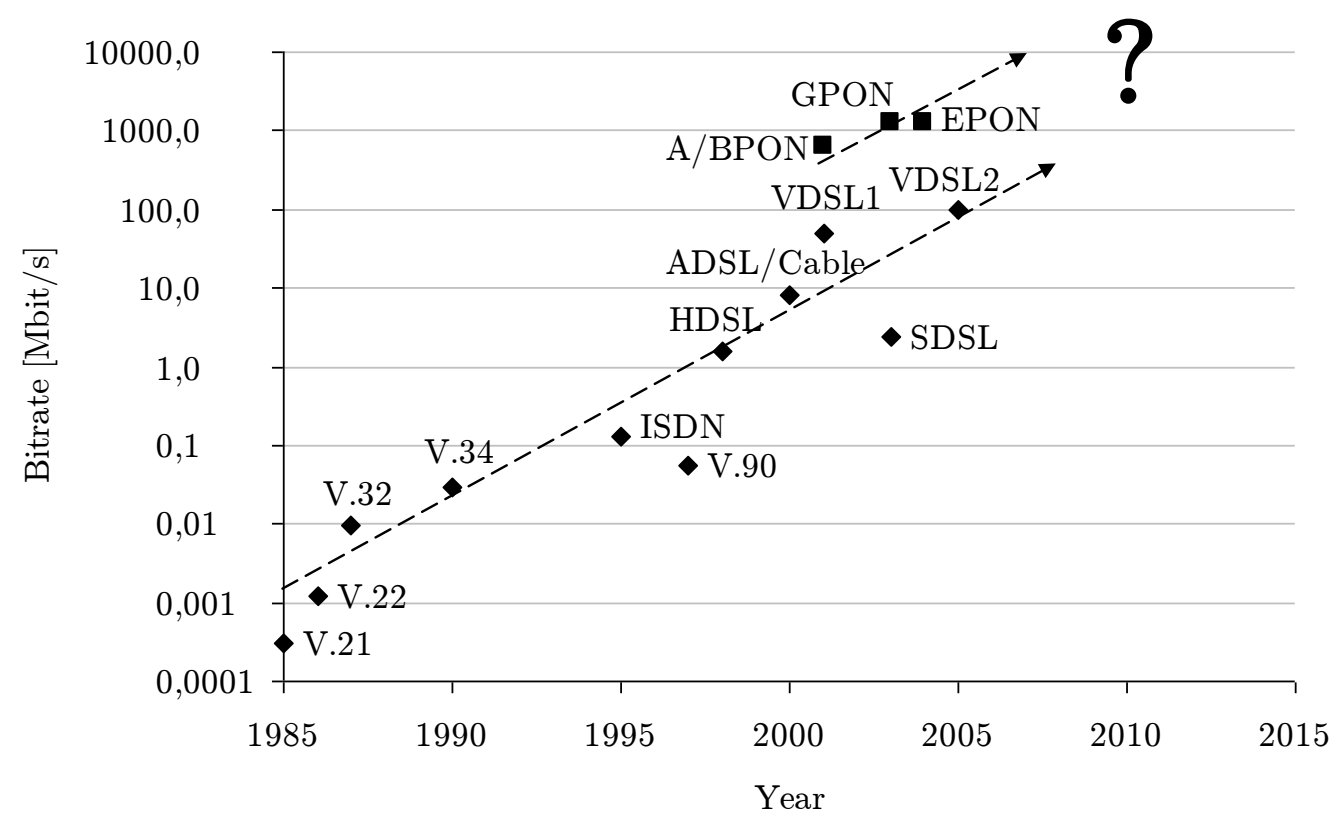

Figure 1.1: Comparison of the evolution of commercially available bitrates in wired access networks (copper and fiber based) for residential users. It must be noticed that the deployed bitrates can be differ significantly in different countries. The specified bitrates are maximum bitrates and depend on many other factors which (can) degrade the specified bitrate significantly. For example, the achievable bitrates in a digital subscriber line (DSL) network depend basically on the distance; in a passive optical network (PON) the bitrate must be shared between all users.

Therefore, further penetration of fiber closer to the customer (from the central office to the street cabinet and further to the home) is inevitable. Fiber access deployment depends on several factors. Each region in the world has its own cultures, economies and unique political situation that influence how, when and why broadband access will be deployed [Whitman04, Hecht04, Lin06]. The following catchwords may also be taken 
into consideration: public policy and regulation, competition, architectures, applications, culture and economic activities [Wolf00, Green03, Hecht04, Whitman04]. Particularly in the case of exhaustive optical access networks, the cost of laying optical cables area-wide is a big challenge. A lot of studies have been done in the past to find cost-optimized cable routes and to find cost-optimized cable laying methods [Bachhiesl04].

Today, a key issue in modern telecommunication networks is the efficient convergence between the internet protocol (IP) and photonic networking. The purpose is to allow for a scalable and cost effective network infrastructure desirable in supporting different Quality-of-Service (QoS) classes [Zheng01]. Especially in WAN and MAN a trend can be seen to minimize the layering complexity and overhead typically produced by Synchronous Optical Network / Synchronous Digital Hierarchy (SONET/SDH) and Asynchronous Transfer Mode (ATM) [Gillespie01, Dixit03]. The intention is to be able to accommodate the Internet packet-based network traffic directly in the optical layer, i.e., WDM layer. The complexity and equipment costs related to electronic multi-layer solutions may be significantly reduced in all-optical WDM networks. The evolution of the layering architecture in terms of telecommunication networks is illustrated in Figure 1.2. It can be seen that the desired solution for future telecommunication networks is an IP-over-WDM architecture [Liu02, Serrat03].

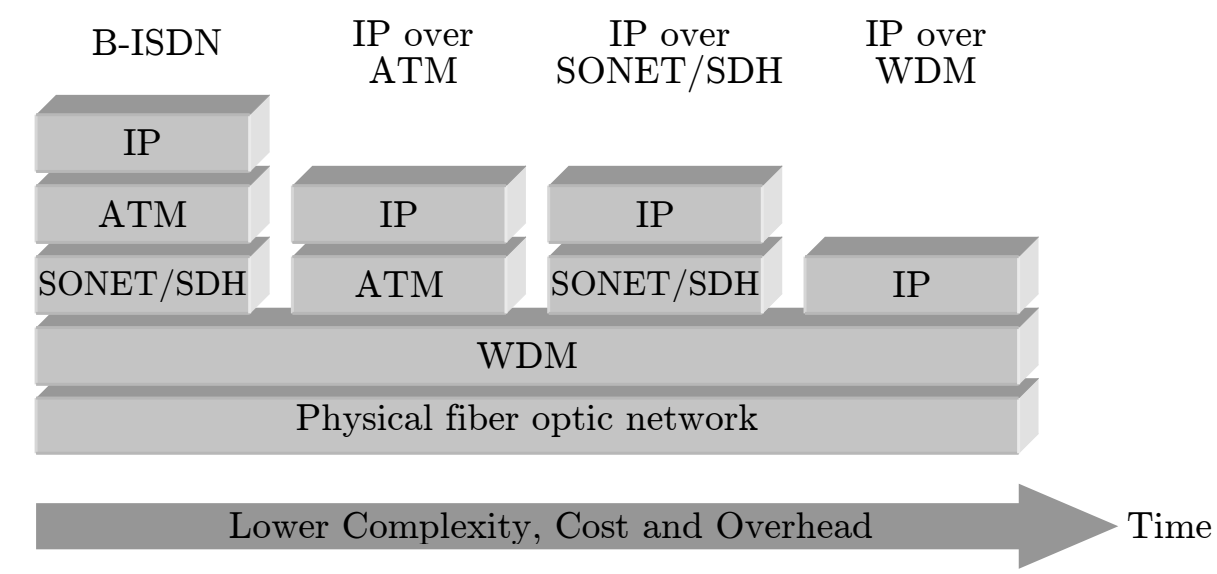

Figure 1.2: Evolution of layering architectures in telecommunication networks. The goal is to reduce the number of needed layers and thus reducing the costs and complexity significantly.

In many countries there is a clear demarcation between the network operators who provide physical connectivity to transport data and the service providers (SP) who deliver the content and the services [Lin06]. To prevent network monopoly by the network operator and to enable free market competition between service providers, it is essential to separate the physical connectivity and the transported services (also known as open access network). Different SPs may be delivering services to the same or a different set of users. In principle, WDM networks offer the separation between the network operator and the SP. The next section provides a general survey on the problems in access networks. 


\title{
1.1 Problems in Access Networks
}

Today, the major challenge is the bitrate bottleneck in access networks due to the ever increasing bitrate requirement per user. In this section, considerations about the bitrate requirements and bitrate limitations were made to give an idea about future requirements. Due to the bitrate limitations, this section answers the following question: is it useful to ever increase the bitrate in networks to reduce the transmission time? Finally, a short overview of state-of-the-art access network technologies deployed in most countries is given.

\subsubsection{Bitrate requirements}

\begin{abstract}
"These days when I watch television here at home, I have my choice of four channels that I can get with reasonable clearness and audibility. Even with only four channels at their disposal, however, the television moguls can supply me with a tremendous quantity of rubbish. Imagine what the keen minds of our entertainment industry could do if they realized they had a hundred million channels (the number of standard television channels that he calculated could fit in the visible spectrum) into which they could funnel new and undreamed-of varieties of trash. Maybe we should stop right now!" [Asimov62].
\end{abstract}

In the past, many projections of future bitrate requirements have been made [Ims98, Coffman01]. I refrained from such forecasts, as they have all proven to be too optimistic. However, it is clear that bitrates will, in fact, continue to grow faster than world population grows. Due to the dropping prices for Internet access (and computers) and as the Internet begins to serve more data-intensive applications, it is also clear that the bitrate requirements per user will grow. Furthermore, the unshakeable demand for bitrates caused by the growth of high bitrate applications and broadband systems leads to higher bitrates requirements per user (Figure 1.3).

\begin{tabular}{llc}
\hline & Application & Bitrate $[\mathrm{Mbit} / \mathrm{s}]$ \\
\hline Video conference (uncompressed) & $4 \mathrm{k}(4096 \times 2048$ pixel $)$ & 6000 \\
Video conference (uncompressed) & $1600 \times 1200$ pixel, 30fps & 1200 \\
Video conference (JPEG 2000) & $4 \mathrm{k}(4096 \times 2048$ pixel) & 400 \\
Storage Area Network & SAN Service & 100 \\
LAN to LAN Connectivity & VPN IP (L3) - Transp. LAN (L2) & 100 \\
HDTV (1080i, compressed) & $1920 \times 1080$ pixel, 30fps & 28 \\
HDTV (720p, compressed) & $1280 \times 720$ pixel, 60fps & 28 \\
Video (uncompressed) & $320 \times 240$ pixel, 15fps & 10 \\
PAL (compressed) & $720 \times 576$ pixel, 25fps & 4 \\
Internet gaming & & 2 \\
CD quality audio & & 1.2 \\
Broadband web surfing & & 0.3 \\
High-quality audio sessions & & 0.125 \\
Voice & POTS / ISDN & 0.064 \\
\hline
\end{tabular}

Figure 1.3: Typical bitrate requirements of different current and future applications [Lund01, Miller02, Simcoe02, Biraghi02, Weldon03, Hecht05, UCSD05]. 
In Figure 1.3, different uncompressed and compressed video services are listed. It must be mentioned that digital compression degrades the signal quality significantly. Therefore, video production and archival storage use lower compression ratios than video transmission (Table 1.1).

\begin{tabular}{lc}
\hline Task & Compression Ratio \\
\hline Video production & $4: 1$ \\
Archival storage & $25: 1$ \\
Transmission & $75: 1$ \\
\hline
\end{tabular}

Table 1.1: Compression levels for high definition television (HDTV) [Hecht05].

In a deployment, not only one service but a service mixture is used and leads to a further increase of the bitrate requirements. [Lund01] points out that a future application mixture leads to a bitrate requirement of approximately $40 \mathrm{Mbit} / \mathrm{s}$ per user. In a residental area with 3,500 supported users, the total bitrate required is roughly $140 \mathrm{Gbit} / \mathrm{s}$. [Lund01] also shows that a $2 \mathrm{~m}$ node rack can service these 3,500 users. Further information about the cable plant design can be found in [Mahlke01].

Another challenge in optical access networks is video transmission. [Goff02] pointed out that many possibilities exist to transmit videos over optical networks. In passive optical networks (PON), there are two preferred methods for video transmission. The first one is the shared transportation within the digital data bit stream and the second one is the transmission over a separate wavelength which is called video overlay [Son03, Zheng03]. The worldwide first development of super high definition (SHD) image technology with 4 times higher resolution than HDTV with $4 \mathrm{k} \times 2 \mathrm{k}=8$ Mega pixels per frame could be shown [Aoyama04]. In a demonstration, the world's first real-time international transmission of super-high definition (SHD) 4k digital video was demonstrated [UCSD05]. The 4k transmission linked the University of California in San Diego to Keio University in Tokyo via $15,000 \mathrm{~km}$ of gigabit Internet Protocol optical-fiber network. Uncompressed streaming of $4 \mathrm{k}$ video requires data rates greater than $6 \mathrm{Gbps}$. In many places the signal must be carried over $1 \mathrm{Gbps}$ circuits. Therefore, a prototype of a joint photographic experts group (JPEG) 2000 codec was designed to compress and decompress the $4 \mathrm{k}$ digital video in real-time to 200 - 400Mbps.

Both examples emphasize the need for high-speed optical access networks. In this work, the considered optical networks can be used to transport video signals within both methods and are also able to transmit video in SHD quality. Therefore, detailed video transmission possibilities are not a topic in this work.

The development of a killer application, for which many customers would be prepared to pay a premium price, could generate a compelling commercial argument for deploying fiber-to-the-home (FTTH) more quickly [Wolf00, Mayhew02]. A possible killer application could be the video transmission in an unforeseen quality.

Symmetry in terms of downstream and upstream bitrates is an important parameter in access networks. The symmetry is defined as the ratio between downstream and upstream bitrates. It was reported that the current symmetry by means of current traffic is approximately 1.4 and is further tending downwards in the future [Reed03]. New and emerging applications such as video conferencing or data file repositories 
require symmetric bandwidth in both directions. In the future, the access network must therefore be capable of transporting symmetrical traffic. Our solutions presented in chapter 3 and 4 are highly symmetric.

In the next subsection, the already existing bitrate limitation and its impact on the transmission time is discussed.

\subsubsection{Bitrate Limitation}

The big question is: does it make sense to ever increase the bitrate in telecommunication networks to reduce the transmission time?

The transmission time depends on two parameters. The first one is the bitrate, which is limited by the transmission channel [Shannon48]. It can be easily calculated by the relation

$$
\text { transmission time }=\frac{\text { file size }[\mathrm{bit}]}{\text { bitrate }[\mathrm{bit} / \mathrm{s}]}
$$

The second parameter is the propagation speed in the medium used. It depends on the used material and can be calculated by the well known equation

$$
\mathrm{v}=\frac{1}{\sqrt{\mu \varepsilon}}
$$

and from this it follows that the transmission time is

$$
\text { transmission time }=\frac{\mathrm{d}[\mathrm{m}]}{\mathrm{v}[\mathrm{m} / \mathrm{s}]} .
$$

For our considerations, we assume a propagation speed of $2 / 3$ of the speed of light, which is valid for the most common transmission mediums.

It must be mentioned that in the real world the transmission time depends on many other parameters like the line coding, protocols, delay times due to the packet switching and so on. These additional parameters enhance the transmission time significantly. Thus, our consideration is reliable, and the published data represents theoretical limits. This means that the transmission time cannot fall below these theoretical limits.

Figure 1.4 gives us an example of how to transfer a 10kByte file over distances between $1 \mathrm{~km}$ and 40,000km. At very low bitrates, the transmission time depends only on the bitrate of the transmission channel, as shown in equation (1.1). If we increase the bitrate, the transmission time drops and approximates the region of the propagation speed. If we further increase the bitrate, at a specific point the transmission time depends only on the limited propagation speed (equation (1.3)). In dependence of the distance and the bitrate, the transmission time depends only on the speed of propagation and not on the bitrate. 


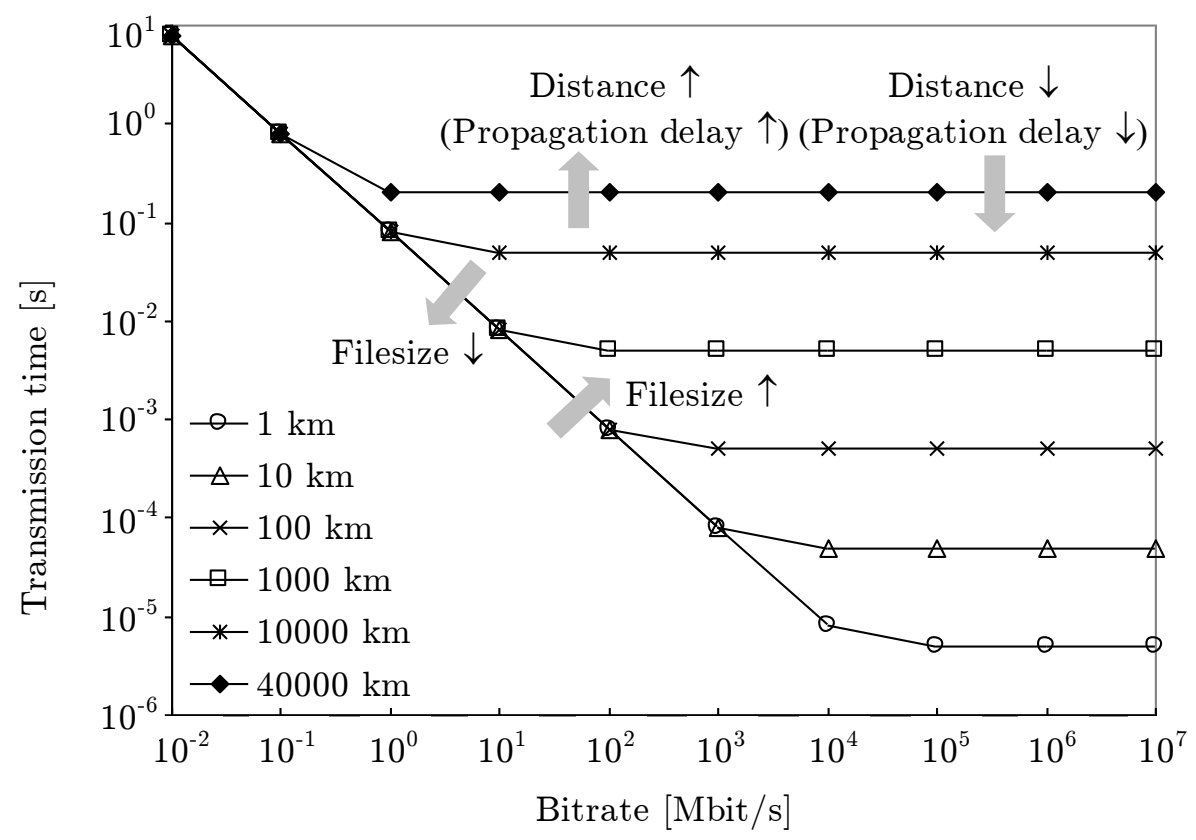

Figure 1.4: Transmission time to transmit and acknowledge a 10kByte file over distances between 1 and $40,000 \mathrm{~km}$. The transmission time declines linearly to a specific point. At this point, the transmission time is constant because it depends only on the velocity of propagation.

Over a distance of $10,000 \mathrm{~km}$, for example, the transmission time goes linearly down with the bitrate, because the transmission time depends linearly only on the bitrate. At approximately $2 \mathrm{Mbps}$, the transmission time remains unchanged due to the constant transmission time that depends only on the speed of propagation.

The transition to the horizontal line depends on the distance and the amount of data which will be transferred. As we can see from Figure 1.4, the horizontal lines show us that the transmission time is limited by the speed of propagation and varies in dependence of the distance (and therefore of the propagation delay). The slanting line depends only on the file size. For larger file sizes, the slanting line moves parallel to greater transmission times and vice versa.

As a consequence, we can see an interesting property: for smaller file sizes, the transmission time depends on the speed of propagation for lower bitrates. That implies that only for larger file sizes is it reasonable to increase the bitrate to reduce the transmission time. 
Figure 1.4 shows the transmission time in dependence on the bitrate and different distances. This diagram can be generalized and is shown in Figure 1.5 for distances between $1 \mathrm{~km}$ and $40,000 \mathrm{~km}$ and for file sizes between 1Byte and 100GByte. With this diagram, we are able to determine the theoretical limitation of the transmission time.

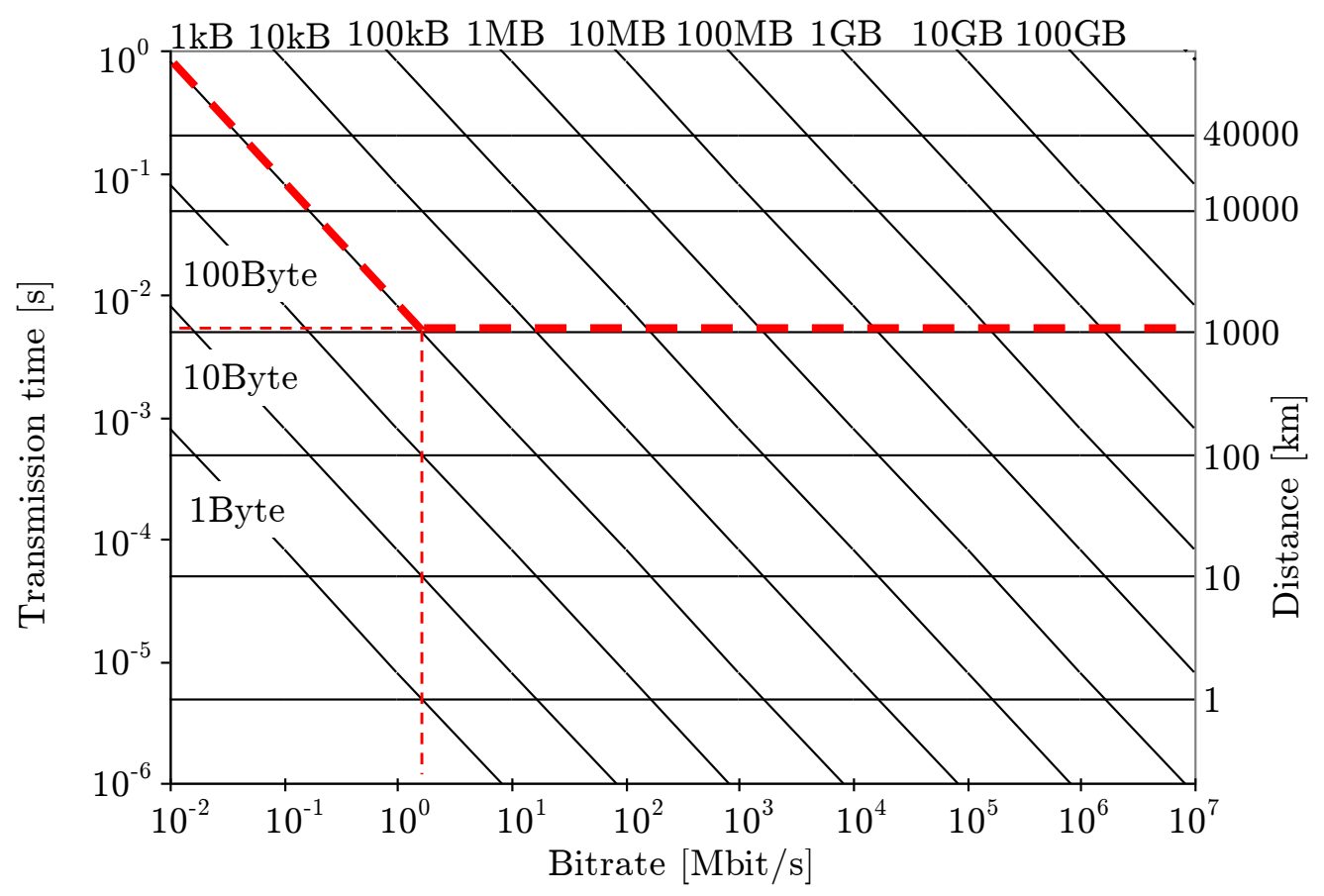

Figure 1.5: Generalized diagram to determine the transmission time in dependance on the bitrate, file size and distance. An example of how to transfer a file with a size of $1 \mathrm{kByte}$ over a distance of $1,000 \mathrm{~km}$ is indicated. In this example, it is obvious that an increase of the bitrate beyond approximately $2 \mathrm{Mbit} / \mathrm{s}$ can not reduce the transmission time. Therefore, the transmission time can not go below $4 \mathrm{~ms}$.

An example in Figure 1.5 is given for a file size of $1 \mathrm{kByte}$ over a distance of $1,000 \mathrm{~km}$. We can see that for bitrates of greater than $2 \mathrm{Mbit} / \mathrm{s}$, the transmission time is constant and cannot underrun the $4 \mathrm{~ms}$ limitation. For this example, it would be pointless to increase the bitrate over $2 \mathrm{Mbit} / \mathrm{s}$. As mentioned before, it must be noticed that the transmission time depends on many other factors and is larger in the real world.

This subsection identified the influencing factors and shows the implications on the transmission time. It could be shown that the transmission time depends on the file size, the distance between the communication nodes and the bitrate of the communication channel (additional parameters like protocols, delay times due to the packet switching, ... are unaccounted). The importance of increasing the bitrates mainly due to the ever increasing data volume has been shown. 


\subsubsection{Current Technologies in Access Networks}

This subsection gives a short introduction to state-of-the-art wired access technologies. The broad field of wireless access technologies is not a topic in this work. The most widely deployed wired "broadband" solutions today are digital subscriber line (DSL) and hybrid fiber coaxial (HFC) networks. Power-line communications (PLC) is always a topic in the access network community but is underpart relating to DSL or PLC networks.

\section{Digital Subscriber Line (DSL)}

The basic premise of the DSL technology is to divide the frequency spectrum into two regions. The lower $4 \mathrm{kHz}$ is used by the plain old telephone service (POTS) equipment, while the frequencies above $4 \mathrm{kHz}$ are being allocated for higher-speed digital communications. Many different types of DSL connections are introduced [Golden08].

The first deployed digital subscriber line, named integrated services data network (ISDN), is capable of transporting digital data with a data rate of $144 \mathrm{kbit} / \mathrm{s}$ [Kanbach99].

The high data rate digital subscriber line (HDSL) is made compatible with a T1 or E1 symmetrical rate of $1.544 \mathrm{Mbit} / \mathrm{s}$ or $2.048 \mathrm{Mbit} / \mathrm{s}$ respectively, for $3 \mathrm{~km}$ or shorter loops.

The symmetric digital subscriber line (SDSL) can have only a symmetric line rate. The rates can be as high as $2.304 \mathrm{Mbit} / \mathrm{s}$ on a 2,4km loop [Chen99, Golden08].

The asymmetric digital subscriber line (ADSL) is the most widely deployed DSL technology [ITU-T G.992.1, ITU-T G.992.2]. In the downstream direction, the line rate could not exceed $1.5 \mathrm{Mbit} / \mathrm{s}$ on a $4,500 \mathrm{~m}$ loop. On shorter loops, the rate can be as high as $6 \mathrm{Mbit} / \mathrm{s}$. In the upstream direction, the line rate can be reach a maximum of $750 \mathrm{kbit} / \mathrm{s}$. Newer standards like ADSL2 specify line rates of up to $12 \mathrm{Mbit} / \mathrm{s}$ for distances less than $1,500 \mathrm{~m}$ and ADSL2+ which defines line rates of up to $24 \mathrm{Mbit} / \mathrm{s}$ on a 1,000m loop [ITU-T G.992.3, ITU-T G.992.4, ITU-T G.992.5]. The actual line rate is chosen by the ADSL modem based on the line condition.

Last but not least, the very high data rate digital subscriber line (VDSL) achieves much higher speed than HDSL or ADSL and can be symmetric or asymmetric. The line rate could range from $13 \mathrm{Mbit} / \mathrm{s}$ for $1,300 \mathrm{~m}$ loops to $52 \mathrm{Mbit} / \mathrm{s}$ for over $300 \mathrm{~m}$ loops [DSLForum01].

\section{Hybrid Fiber Coaxial (HFC)}

Mainly two standards are present in hybrid fiber coaxial (HFC) networks [Frohberg01]. From the USA the data over cable service interface specification (DOCSIS) with a television (TV) channel bandwidth of $6 \mathrm{MHz}$ and transparent bidirectional transmission (EuroDOCSIS is the European pendant with a channel bandwidth of $8 \mathrm{MHz}$ ). DOCSIS provides a downstream line rate between $30.3 \mathrm{Mbit} / \mathrm{s}$ and $42.9 \mathrm{Mbit} / \mathrm{s}$ and upstream line rates between $320 \mathrm{kbit} / \mathrm{s}$ and $10.240 \mathrm{Mbit} / \mathrm{s}$.

From Europe, the DVB-RCC standard (digital video broadcasting return channel for cable) with a channel bandwidth of $8 \mathrm{MHz}$ allows for a downstream line rate between $1.544 \mathrm{Mbit} / \mathrm{s}$ and $55.6 \mathrm{Mbit} / \mathrm{s}$ and an upstream line rate between $256 \mathrm{kbit} / \mathrm{s}$ and 12.352Mbit/s as specified in ETSI-Standard ETS 300800. 
DVB-RCC specify frequencies between $70 \mathrm{MHz}$ and $862 \mathrm{MHz}$ for downstream and frequencies between $5-65 \mathrm{MHz}$ for the upstream direction. For the downstream direction DOCSIS use frequencies between $50 \mathrm{MHz}$ and $860 \mathrm{MHz}$ and frequencies between $5 \mathrm{MHz}$ and $42 \mathrm{MHz}$ for the upstream signals.

\section{Power-line Communication (PLC)}

The key asset of Power line communications (PLC) is "no new wires" [Ferreira95]. Power grid networks usually form a bus or tree topology in which all users must share the available bandwidth. Due to their open and insecure bus structure, power-line networks presents a number of security challenges [Dostert01].

Related to standards and regulatory issues, there are many activities in Europe and in the United States [Pavlidou03]. These standards regularize frequencies up to $525 \mathrm{kHz}$ in Europe and up to $30 \mathrm{MHz}$ in the United States. One of the major issues is the radiation emission of power line networks.

Outside of the international standardization body, non-profit corporations like [Homeplug] create open specifications for high-speed home power line communications. In the HomePlug Standard 1.0, line rates of up to 14Mbit/s and with the HomePlug AV standard line rates of over $100 \mathrm{Mbit} / \mathrm{s}$ are possible.

Current activities in the European Union like the open PLC European research alliance (OPERA) promises line rates of up to 200Mbit/s [OPERA].

The theoretical maximum data rate can be estimated with the well-known Shannon's theorem

$$
\mathrm{C}_{\mathrm{c}}=\mathrm{B} \cdot \log _{2}\left(1+\frac{\mathrm{S}}{\mathrm{N}}\right)
$$

whereas $\mathrm{C}_{c}$ represents the maximum data rate in bits/s for a theoretically error-free transmission. $\mathrm{B}$ is the available bandwidth and $\mathrm{S} / \mathrm{N}$ is the signal-to-noise ratio at the receiver's input. Channel estimation shows that a theoretical data rate between $14 \mathrm{Mbit} / \mathrm{s}$ and $250 \mathrm{Mbit} / \mathrm{s}$ are achievable with link lengths of maximum 300m [Dostert01 p.269-273].

Power line networks have a niche market in remote areas served by power cables [Pavlidou03]. If such line rates works for some time, the biggest question remains unanswered: is this technology competitive in quality, speed and price with services already offered by digital subscriber lines or cable modems?

\section{Discussion}

Over the near term, DSL, PLC and HFC networks offer viable bitrates for conventional applications. Residential customers can choose between these wired technologies. All these technologies are limited in their range and their performance. As traffic requirements increase, new technologies in access networks are required [Lin06]. In the near future, broadband access networks will be required with data rates over $1 \mathrm{Gbit} / \mathrm{s}$ for each customer [Söderlund04]. Due to this reason, the widespread deployment of fiber-tothe-home (FTTH) technology is speeding up.

In summary, the existing "broadband" solutions are unable to provide enough bandwidth for emerging sevices such as video conferencing, video-on-demand (VoD), or interactive gaming [Wolf00, Lin06]. Future access networks must be able to transport all services over a single line of communication. 


\subsection{Organization of this Work}

This work is organized in six chapters (Figure 1.6) and is based on the knowledge of cognitive learning methods [Schussmann07b]. Besides this motivation part, a comprehensive overview of state-of-the-art optical access networks is given in chapter 2 . Here, our consideration is focused on single-hop networks based on passive star and tree topology. The functionality and the limitations of time division multiplexed passive optical networks (TDM PON) are shown. This leads to the next generation passive optical networks which are based on wavelength division multiplexing. Beside the technological background of basic components, problems and possible state-of-the-art solutions for down and upstream transmission, important standards are discussed.

In section 2.5, we present the paradigm shift and its characteristics in future optical access networks. The basic statement of the paradigm shift is to provide each user and each service with at least one wavelength.

In chapter 3, basic properties of single-stage networks based on an arrayed waveguide grating multiplexer (AWGM) are presented and analyzed via wavelength transfer matrices (WTM). Next, the WTM has been generalized to characterize single-stage networks with coarseness factors greater than one. Thereafter, network design considerations allow for the design of a single-stage network based on the paradigm shift. Finally, the connectivity map shows us the correct functionality of the network design methods. Insuperable limitations of single-stage networks will lead to the need for multistage WDM PON in the next chapter.

In chapter 4, design considerations of multistage networks which fulfill the paradigm shift in section 2.5 are proposed. First, we present the considered methods for modelling multistage networks. Next, all relevant design parameters are identified and then classified in design dependent and independent parameters to give us an idea of such networks. Detailed examinations of design dependent parameters give us a better understanding of multistage networks. Further on, two approaches for calculating the connectivity map are presented. In the first one, all wavelengths are predetermined in the central office (CO) and are calculated through the whole network up to the users.

The second method allows to predetermine desired wavelengths on the user's side, and then the wavelengths are calculated stage by stage in the direction of the CO. Furthermore, a power budget calculation for multistage WDM PONs allows us to determine the correct functionality and the maximum expansion in terms of power budget limitations.

In chapter 5, all realizable architectures which fulfill the design conventions in subsection 4.1 .1 can be generated by a developed design tool. The goal was to identify those architectures which minimize the costs. Different cost functions which take into account the wavelength router (WR), cable and installation costs were defined. Another issue in a deployment is the position of the WR in a multistage WDM PON. Therefore, the cost impact of the WR position was analyzed by the method of virtual movement of the WRs to better understand this influencing parameter. 
In chapter 6, the implementation of a future-proof multi-stage WDM PON network is shown. The current expansion of the WDM PON allows for the transmission of 10 wavelengths in both directions in a three-stage architecture. This network acts as a platform and supports the implementation and demonstration of future (killer) applications. Many different future applications can be demonstrated on this platform. The most impressive application is a real-time video conference in the highest high definition television (HDTV) quality. This application was implemented on standard Windows $\mathrm{XP}^{\circledR}$ personal computers. To demonstrate the unimaginable quality, two THX-sound systems with a Holo-Pro system (transparent freestanding projection screen with an extremely high image resolution) and a large screen were installed. We are now in the position to present the enormous information transmitted in a unique quality.

Chapter 7 provides conclusions as well as an outlook on further research activities. In Appendix A, the list of abbreviations used in this work is itemized, while Appendix B delivers the list of the most commonly used mathematical symbols within this work. In Appendix C, the list of standards related to this work, is itemized.

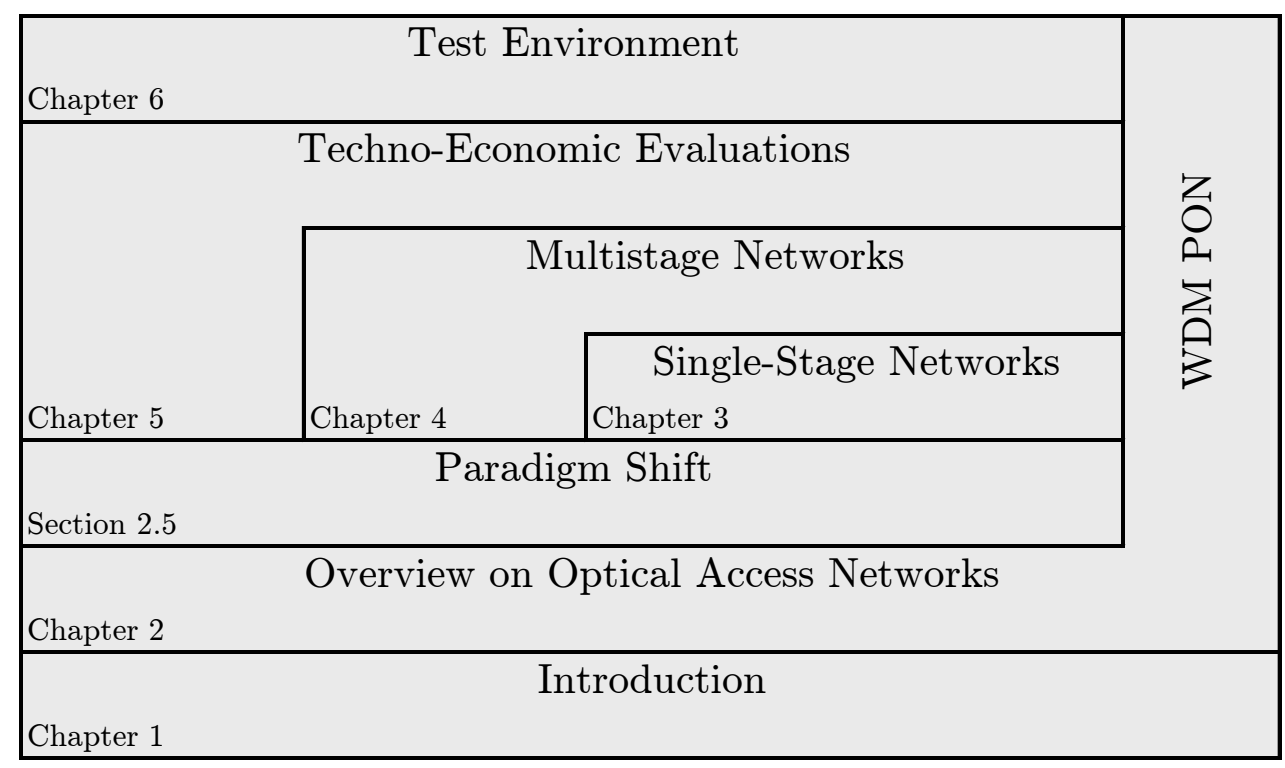

Figure 1.6: Structure of this work divided into six chapters. 


\section{Overview on Optical Access Networks}

his chapter gives an introduction and a technical overview of the required
components and multiplexing techniques to set up state-of-the-art optical access
networks (OAN).

\subsection{Classification of Optical Access Networks}

An access network is defined as the set of equipment and infrastructure needed to perform the connection between the carrier premise and the user's equipment [Prat02]. Access networks are also denoted by the phrase "first mile", "last mile" or "local loop". The phrase "first mile" reflects the importance and high priority of access networks due to the existing bitrate bottleneck.

There are a lot of possibilities to classify optical access networks [Greenfield02, Stern02]. In the next sections, different classifications are accounted for classifying this work. A common taxonomy is the used optical penetration. Figure 2.1 illustrates different optical penetrations from Fiber-to-the-Cabinet (FTTcab) to Fiber-to-the-Home (FTTH).

In each option, the core network is connected to the optical line termination (OLT) via a network node interface (NNI). Today, the NNI is typically a SDH / SONET interface [Andersen97, Greenfield02]. Between the OLT and the optical network unit (ONU), standard single mode fibers $(\mathrm{SMF})$ were normally used to transmit the information optically. Due to different fiber penetration possibilities, the ONU is located at different locations. In the case of FTTcab, the ONU is situated in the cabinet, in FTTC at the curb, and in the case of FTTB, in the building. For these cases, the ONUs are connected to a network terminal (NT) over short copper connections (typically xDSL). In FTTH networks, the fiber penetration is close to the user. Only in this case, optoelectronic conversion within the network is not necessary, which results in lower costs, higher transmission quality and higher bit rates. Users are connected via the user network interface (UNI). Today, the user network interface is implemented as an Ethernet, universal serial bus (USB), wireless local area network (WLAN) or other interfaces. 


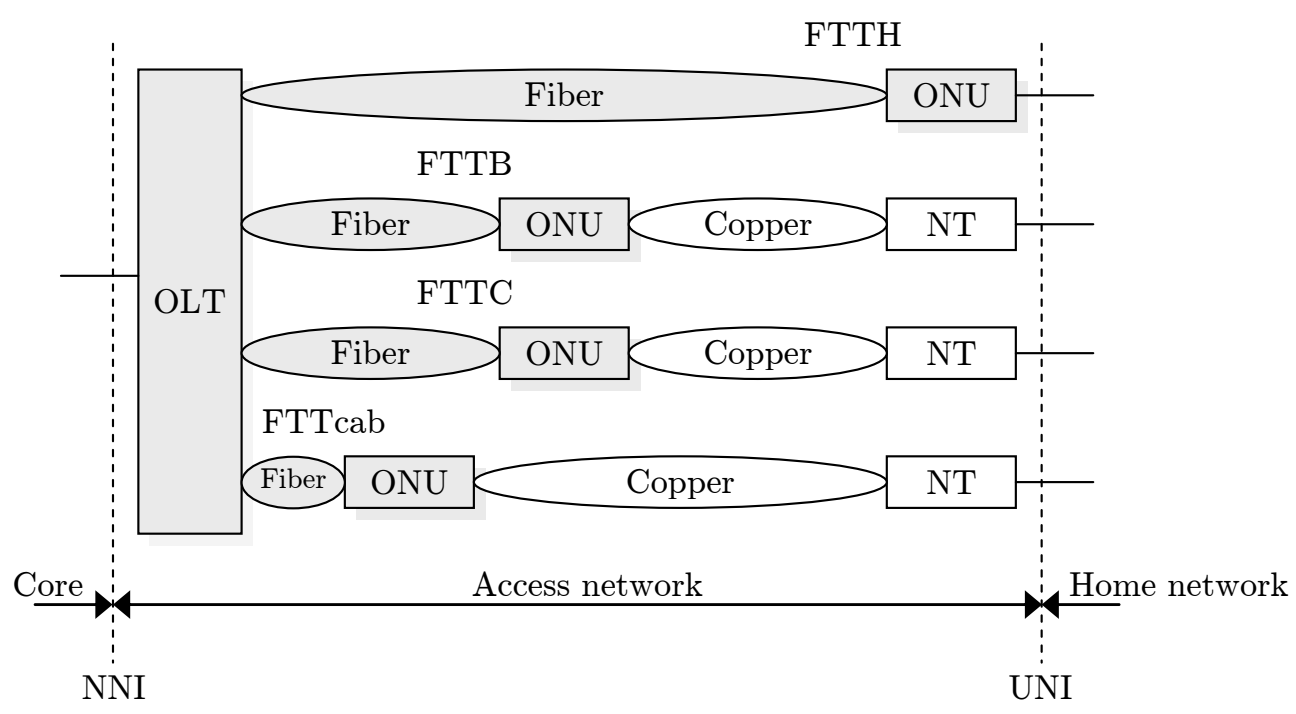

Figure 2.1: Different optical access network architectures in dependence on the optical penetration from FTTcab to FTTH. The access network is connected via a network node interface (NNI) (typically SDH / SONET) to the core network and with a user network interface (UNI) (typically Ethernet, ISDN, ...) to the home network. Only in the case of FTTH, the maximum possible fiber penetration is available. In the other cases, the gap is bridged by copper links between the optical network unit (ONU) and the network termination $(\mathrm{NT})$.

Another frequently used taxonomy is the utilization of active or passive components inside the optical access network. Thus, the optical parts of access networks fall into one of the following categories [Voges02, pp. 943-955]:

- active architectures

- passive architectures.

The main advantage in active architectures is the dedicated link between the ONU and the OLT. The outcome of this fact is the usage of cheaper components in the ONU compared with passive architectures. In active architectures there are several issues to be taken into account: lack of transparency for different bit rates and modulation techniques, heavy environmental conditions for the equipment, additional costs for maintaining the active devices and the required power supply.

A European example for an active architecture is the Optisches Anschlussleitungssystem (OPAL) of the Deutsche Telekom, which is an active optical network mainly deployed in the newly formed German states [Pospischil95, Frohberg01]. It provides user line rates from $64 \mathrm{kbit} / \mathrm{s}$ up to $2.048 \mathrm{Mbit} / \mathrm{s}$. In the old West German states, the "Integriertes System zur Bereitstellung der Netzinfrastruktur auf optischer Basis" (ISIS) was introduced and the architecture is nearly the same as in the OPAL network [Pospischil95].

In networks with passive architectures, the passivity arises from the design contrast with metro networks in which switching, amplification, and regeneration of the optical signal is carried out en-route from a source to a destination [Ramamurthy01]. Therefore, passive architectures typically do not require active optical components, namely switches, amplifiers, and so on, which are the key to the upward-driven prices seen in generic optical networking. In networks with passive architectures, all ONUs must deal 
with the total bandwidth of the optical channel which leads to increased costs for the ONUs. A passive architecture is completely transparent for bit rates and modulation techniques and only ONUs need the new capacity features. Due to the extreme cost sensitivity of access networks, the objective is to avoid active components. Moreover, future proof solutions require only passive components. On the basis of the avantages of passive architectures and the higher costs of active architectures, this work focuses on access networks with passive components.

Furthermore, optical access networks can be classified into

- single-hop networks

- multi-hop networks.

In a single-hop network, information is sent directly from the source node to the destination node without being routed through intermediate nodes. In a multi-hop network, the data transmitted from a source node to a destination node may be routed through the electronics at intermediate nodes in the network, i.e., the transmitted packets leave the optical domain for a short period of time in order to be routed [Stern02].

In other words, single-hop networks obtain optical transparency, optical self-routing and all-optical communication between nodes. Additionally, in contrast to multi-hop communications, the node processing requirements are kept relatively slow. That is because nodes must only process data packets destined to themselves compared to multi-hop networks in which a node must contingently store and forward data packets to intermediate nodes.

In the case of optical access networks, there is a great number of ONUs which leads to a high cost sensitivity for that devices. In multi-hop networks, the node (ONU) processing requirements stated before leads to higher cost. As a result of the cost sensitivity, this work focuses on single-hop networks.

In addition, WDM networks can also be classified into

- broadcast-and-select networks

- wavelength-switching networks

- wavelength-routing networks.

In a broadcast-and-select network, the network is completely passive and does not include any internal wavelength sensitivity. Therefore, only the end nodes are responsible for selecting the suitable wavelength. In a wavelength-Switching network, wavelength switching is performed by wavelength conversion [Mukherjee97, p. 341 et seq., Mohammad03, p. 433 et seq.] or dynamically by switching from one path to another. In a wavelength-routing network, a specific wavelength determines the route between the source and the destination node. In both, wavelength-switching and wavelength-routing networks, wavelength-selective devices are needed within the network. Due to the heavy cost conditions of wavelength conversion and wavelength switching, this work focuses on broadcast-and-select networks (section 2.3) and wavelength-routing networks (as of section 2.4). 
The fundamental purpose of any communication network is to provide connectivity between pairs of nodes. That implies two kinds of communication network architectures. In networks based on a broadcast architecture, one sender sends the signal to the network which sends the received signal to all other nodes. Thus, no routing function is provided by the network. Most local area networks (LAN) follow the same principles as the Ethernet. Optical networks based on a passive star coupler (PSC) are broadcast architectures and are discussed in section 2.3.

The other type of network architecture selectively transmits the signals received from one node over a part of the network. The distributed routing function is performed by the network nodes (wavelength-routing). Today, most wide area networks are based on this category and are usually interconnected by point-to-point links. Optical networks which are based on static wavelength routers fit into this category of networks and are the main parts of this thesis.

Optical access networks may also be classified by the used topology. In general, networks are commonly based on

- $\quad$ star

- bus and

- $\quad$ ring

topologies [Stern02, Gumaste04]. Ring topologies are mainly used in metropolitan area networks (MAN) and wide area networks (WAN) due to their excellent protection and restoration possibilities, which are not required in access networks. In practice, two different topologies are used for broadcast architectures, namely the star and the bus topology as shown in Figure $2.2 \mathrm{a}$ ) and b). Both topologies use passive star couplers (PSC) to split the optical signal which results in copies of the information. Star couplers with multiple input and output ports, as shown in Figure 2.2, can be manufactured by a single integrated device or by interconnecting several $2 \times 2$ (3-dB) couplers (see subsection 2.3.1). As we can see in Figure 2.2, the topologies differ in the number and manner in which the couplers are used. A bus network with $\mathrm{n}$ nodes uses a number of 2(n-1) 3-dB couplers. A star network with $\mathrm{n}$ nodes uses a single $\mathrm{n} \times \mathrm{n}$ star coupler which can be manufactured with a number of $\frac{\mathrm{n}}{2} \log _{2} \mathrm{n} 3$ - $\mathrm{dB}$ couplers, whereas $\mathrm{n}$ must be a power of 2. The main drawback of the bus topology is the number of $3-\mathrm{dB}$ couplers that a single signal must pass through. In the worst case, a number of 2(n-1) PSCs must be passed, whereas in the star topology it always uses a number of $\log _{2} n$ couplers. This plays a significant role in determining the link budget performance of these topologies (see subsection 2.3.1).

To alleviate the link budget performance problem, optical amplifiers were introduced. An amplification of more than $20 \mathrm{~dB}$ was achieved in a spectral range of $1551 \mathrm{~nm}$ to $1611 \mathrm{~nm}$ by using a 4-Channel coarse wavelength division multiplexing (CWDM) amplifier [Hayashi04]. This amplifier allows to extend the distance between the OLT and the ONUs significantly but it is highly cost-intensive. Because of the cost sensitivity in access networks, optical amplifiers are not an issue today. 
a)

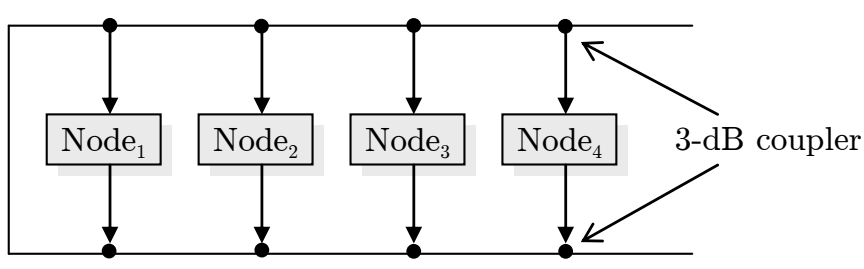

b)

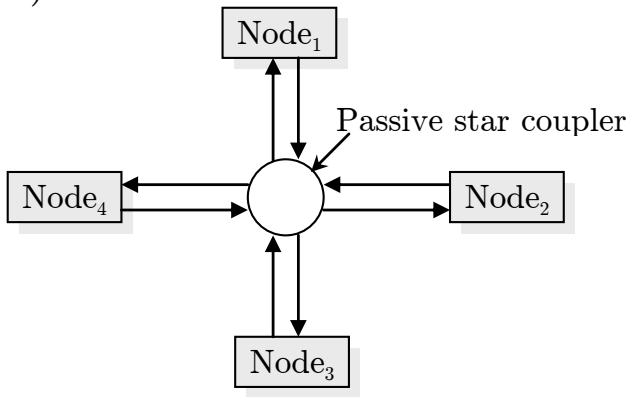

Figure 2.2: For broadcast-and-select optical access networks, two topologies are practicable: a) the bus and b) the star topology. By means of the power budget performance, the star topology is the preferred embodiment topology today (see subsection 2.3.1).

Traditional and present wired (copper) access networks like xDSL can typically be viewed as a single channel network. In most cases, the optical fiber medium is simply used as a replacement for copper cables to enable the system to operate at higher distances and bit rates. Today's access networks are mainly used to transport one service. Commonly, they work with transmission rates of less than $10 \mathrm{Mbit} / \mathrm{s}$. The objective is a network which is called open access network. An open access network is a network established by a network (or infrastructure) operator to enable delivery of advanced services. Multiple service providers must be able to deliver multiple services to users across the network for a fee. Basically, there are several multiplexing techniques to realize multi provider / multi service optical access networks, namely

- $\quad$ SDM (Space Division Multiplexing)

- TDM (Time Division Multiplexing)

- WDM (Wavelength Division Multiplexing)

- CDMA (Code Division Multiple Access)

- PPS (Photonic Packet Switching)

○ OTDM (Optical Time Division Multiplexing)

- Burst Switching.

Logical channels can be obtained by partitioning space, time, wavelength, code or any combination of there component. Although some first system design considerations exist, Photonic Packet Switching (PPS) networks are mainly discussed in local/metro networks and can still be viewed as premature technologies compared to the other listed techniques.

In PPS networks, the packet-switching function is performed optically. The goal of PPS networks is the same as with electronic packet-switched networks, but at much higher speed (switching traffic in the order of ten to hundreds of Tbit/s at line rates of 80Gbit/s and beyond). PPS networks can be classified into Optical Time Division Multiplexing (OTDM) and burst switching networks. OTDM networks have the same functionality as electronic TDM networks. The difference is that the multiplexing and demultiplexing operations are performed optically at higher speeds (typical aggregate bitrates are on the order of 100Gbit/s) [Ramaswami02]. In burst switching networks, the source node transmits a header (typically at a lower speed and out-of-band) followed 
by a packet burst. An interjacent node receives the packet header and activates its switch to connect the following packet burst stream to the suitable output port. If the output port is not available at the moment, the packet burst can either be buffered or dropped.

The main difference between OTDM and burst switching networks is the packet length. In burst switching networks, the packet length can be fairly long, compared with OTDM [Ramaswami02].

Code Division Multiple Access (CDMA) is another possible method for multiplexing and has been successfully introduced in the Universal Mobile Telecommunications System (UMTS). In this technique, a unique code sequence (Chips) assigned to each channel is used to encode the low-speed data, which is then multiplexed in a single fiber. The frequency spectrum of the code sequence is much larger than the signal bandwidth which results in a huge network capacity. In fiber optic networks, dispersion influences the CDMA operation leading to moderate bitrates in the area of a few Mbit/s [Ahn02]. Another constraint is the requirement to generate ultra-short pulses synchronized to the desired channel [Dutta04, p. 277]. As a result, CDMA is not a topic of this work.

In the next sections, promising multiplexing techniques are discussed in detail. The goal is to assess the network capacity limits and the pros and cons. 


\subsection{SDM}

In an optical access network, the Space Division Multiplexing (SDM) technique leads to Point-to-Point links between the OLT and the ONU. The most important point-topoint technology is Ethernet as the link layer protocol [Mickelsson04]. It is a futureproof technology because the fully symmetrical high bandwidth of the fiber is available for future upgrades. For long distance transmission, a high power budget is available. An important drawback is the lack of broadcast services [Mickelsson04].

Space division multiplexing enables a simple provider independent pay-as-you-grow strategy (multi service solution). Due to the lack of passive components, relaxed specifications for the optical components enable low cost solutions [Mickelsson04].

One perceived shortcoming of point-to-point links is the high fiber count and the resulting problems created when terminating the fibers in a central office. [Mickelsson03] shows that this problem can be solved by using ribbon technology in combination with fiber optic array modules.

SDM was not standardized before the IEEE802.3 group initiate the Ethernet in the First Mile [EFM] task force in September 2001. Since mid 2004, standards exist for xDSL, Ethernet passive optical network (EPON) (see subsection 2.3.2) and Point-toPoint fiber. Furthermore, it also specifies operation, administration and maintenance (OAM) functionality for the link layer. EFM addressed the gaps that exist in the Ethernet family when applied to broadband access applications.

Point-to-Point solutions have an enormous drawback; the amount of needed fibers and therefore the enormous trenching cost and the number of needed transceivers. Unbundling, which is an important property to deploy a new access network technology, is possible with the drawback that we need two fibers for each service or provider.

Today, other technologies like Ethernet in combination with SDM are not really present.

In the next section, the technological background needed to build up TDM PONs are explained. State-of-the-art technologies and up-to-date standards are discussed. 


\subsection{TDM PON}

In this section, the required passive components for building time division multiplexed passive optical networks (TDM PON) and the capacity limits of such networks are discussed. An introduction of state-of-the-art standardizations gives us an overview of the current state. The goal of each network provider is to account for each carried bit. Due to the protocol overhead, bits that cannot be accounted must be also transfered. As a consequence, the accounted bits (revenue bits) should be maximized or in other words, the overhead should be reduced. Thus, the comparison of the impact of overhead bits for different layer 2 network technologies is discussed.

\subsubsection{Technological Background}

The basic component to build-up a TDM PON is the passive star coupler. The term coupler covers all devices that split light out or combine light into a fiber [Mestdagh95, Que02, Agrawal02]. A splitter is a coupler that divides the optical signal from one fiber to a minimum of two fibers. The most common splitter is a $1 \mathrm{x} 2$ splitter (see Figure 2.3(a)). The splitting ratio $\alpha$ is the fraction of input power that goes to each output and can be manufactured with any ratio [Kashima95, Goure02, p. 75 et seq., Lin04]. Splitters are the opposite of combiners, and when turned around, a splitter can be used as a combiner (see Figure 2.3(b)). In general, a $2 \times 2$ coupler can be made up of a $2 \times 1$ combiner followed by a $1 \times 2$ splitter (see Figure 2.3(c)). The coupler has the effect of broadcasting the signals from two input fibers onto two output fibers.

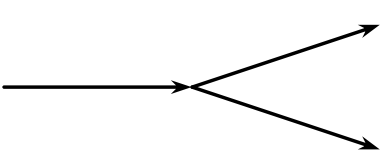

(a) splitter

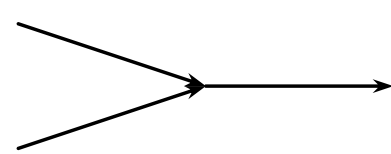

(b) combiner

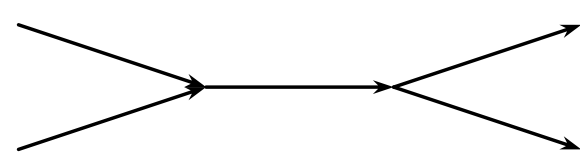

(c) coupler

Figure 2.3: Basic passive optical components: splitter, combiner and coupler.

The central device in a passive-star configuration is the optical star coupler itself. It is also known as passive star coupler (PSC). The most popular way to form a passive-star coupler is to put together many stages of individual $2 \times 2$ fused single-mode fiber couplers (Figure 2.5). Two single-mode optical fibers are fused together with their cores slightly separated (Figure 2.4). When optical power is launched on port 1, then a fraction appears on port 3 and on port 4 . In practical couplers, a small fraction of the power is lost inside the coupler and another small fraction of the launched power is reflected back.

It is essential that the optical power coupled from one fiber to the other can be varied by changing the length of the coupling region where the fields from the two fibers interact [Ghatak98, p. 361 et seq., Bludau98, p. 321 et seq., Chang03, p. 707 et seq.]. The parameters of the coupler can be adjusted such that the power division at the output port is exactly $1 / 2$, given a $3-\mathrm{dB}$ coupler. 


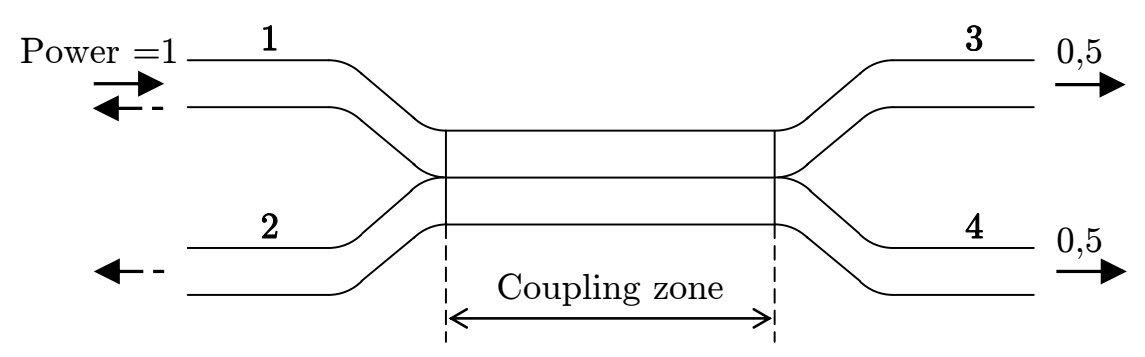

Figure 2.4: A 2x2 fused coupler obtained by melting and pulling two single-mode fibers together over a coupling zone with $3-\mathrm{dB}$ power splitting.

In practice, passive devices always have excess losses resulting from insertion losses, intrinsinc losses in the constitutive coupler medium, and output coupling losses.

The excess loss can be represented by the coupler transmission factor $\kappa(0 \leq \kappa \leq 1)$ which is defined as the fraction of the total input power that appears as total output power ( $\kappa=1$ means no loss and $\kappa=0$ means pure loss). Typical excess losses, expressed in decibels, are $\kappa \approx 0.1$ to $0.5 \mathrm{~dB}$.

In a further step, the 3 - $\mathrm{dB}$ coupler can be connected to form a passive-star coupler [Mestdagh95, Wan00, p. 41 et seq.]. Figure 2.5 depicts a possible realization of a $16 \times 16$ star coupler. To build a $\mathrm{NxN}$ passive star coupler, it can be derived that $\frac{\mathrm{N}}{2} \log _{2} \mathrm{~N}$ couplers have to be employed with $\log _{2} \mathrm{~N}$ stages. The total loss of the star coupler is taken into account by $\kappa^{\log _{2} \mathrm{~N}}$. The output power appearing in the passive star couplers outputs can be written as

$$
\mathrm{P}_{\text {out }}=\mathrm{P}_{\text {in }} \frac{1}{\mathrm{~N}} \kappa^{\log _{2} \mathrm{~N}}
$$

For $\kappa=1$ (no coupler losses), the output power obviously becomes $\mathrm{P}_{\text {out }}=\frac{\mathrm{P}_{\text {in }}}{\mathrm{N}}$. In practice, of course, the output power division is not equal because the output power distribution may vary depending on the signal-carrying input port.

Another important fact resulting from the cascading of $3-\mathrm{dB}$ couplers is that $\mathrm{N}$ is a multiple of 2. This affects the flexibility because when just one additional ONU is connected to the already fully connected $1 \mathrm{xN}$ star network, the $1 \mathrm{xN}$ star coupler must be replaced by a $1 \times 2 \mathrm{~N}$ coupler; thus leaving $\mathrm{N}-1$ ports unused. Alternatively, when $\mathrm{N}$ is not a power of two and $\mathrm{N}$ is large, a star coupler implementation based on an arrayed waveguide multiplexer device is to be preferred [Kaminow02a]. 


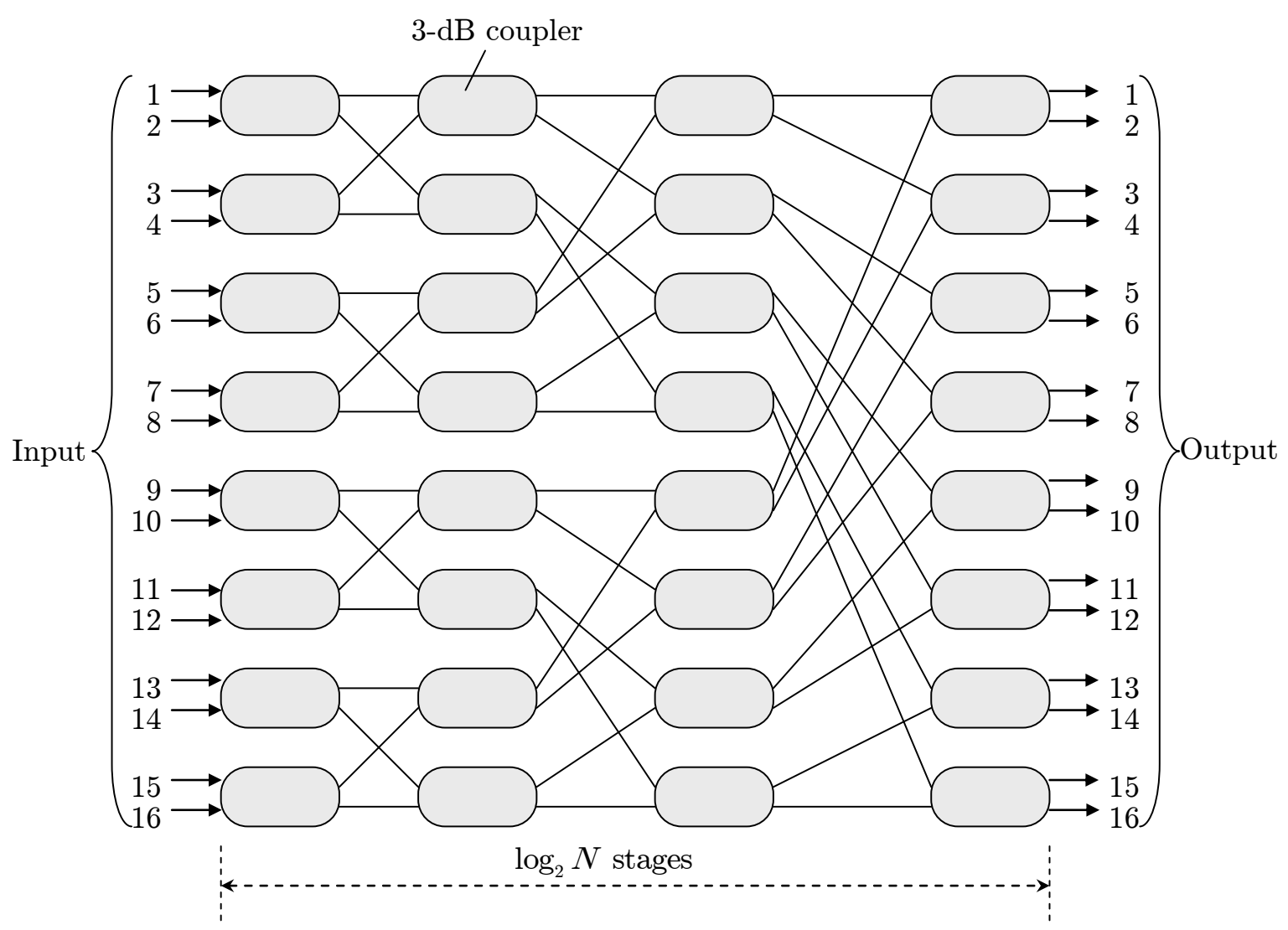

Figure 2.5: Example of a $16 \times 16$ star coupler configuration. The required number of 3 -dB couplers can be easily calculated by $\frac{\mathrm{N}}{2} \log _{2} \mathrm{~N}$. Therefore, 323 -dB couplers are required to build up this $16 \mathrm{x} 16$ star coupler.

In summary, the resulting star coupler made up of fused fiber couplers can be characterized by

- low insertion loss,

- low reflections,

- long term stability,

- low material costs,

- $\quad$ easy manufacturing, and, therefore, commercial availability.

A drawback of the fused fiber coupler is the little potential for integration. In addition, when $\mathrm{N}$ is not a power of two and is large, then a coupler implementation based on an arrayed waveguide multiplexer is preferred (see section 2.4). 


\section{Capacity Limits}

It is helpful to know the different influencing factors that limit the capacity in a network [Bengi02]. We define the capacity limit of a network to be the product of the channel bitrate on each source or wavelength $\mathrm{B}_{0}$ and the number of sources or wavelengths $\mathrm{N}_{\omega}$ where

$$
\mathrm{B}_{\mathrm{N}}=\mathrm{B}_{0} \cdot \mathrm{N}_{\omega}
$$

Basically, there are several limitations to this network capacity of a passive star system; namely

- electronic transmission and reception speed,

- power budget limit,

- optical spectrum access.

The electronic transmission and reception speed of each node is limited. This is performed by current electronics technology. Today, bit rates of up to 40G Git/s can be achieved and are practical. It is not surprising that the network capacity is increasing linearly with the number of wavelengths. Due to the ever-increasing transmission speeds in electronics technology, the considered channel bit rates in Figure 2.6, are 100Mbit/s, $155 \mathrm{Mbit} / \mathrm{s}, 622 \mathrm{Mbit} / \mathrm{s}, 1 \mathrm{Gbit} / \mathrm{s}, 2.5 \mathrm{Gbit} / \mathrm{s}$ and $10 \mathrm{Gbit} / \mathrm{s}$. Clearly, there is nothing very fundamental about this limit as they are limited only by the ever-increasing speeds of electronic technology.

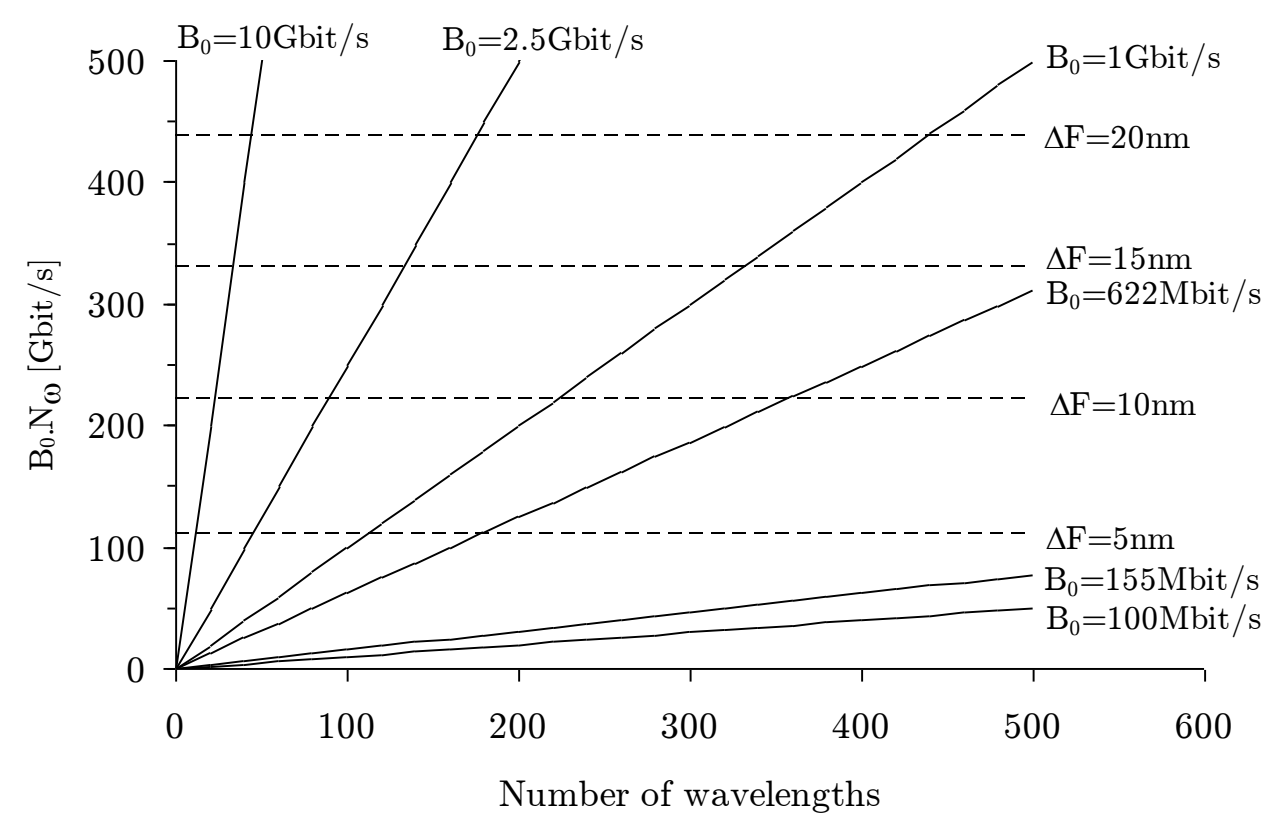

Figure 2.6: Network capacity limits of a WDM passive star network due to electronic transmission and reception speed (straight lines) and optical spectrum access (dashed lines). $\mathrm{B}_{0}$ : channel bit rate; $\mathrm{N}_{\omega}$ : number of wavelengths; network capacity: $\mathrm{B}_{\mathrm{N}}=\mathrm{B}_{0} \cdot \mathrm{N}_{\omega} ; \Delta \mathrm{F}$ : accessible frequency spectrum.

The second limiting factor is the power budget limit. This is imposed by the maximum available source power and the minimum reachable receiver sensitivity [Agrawal02, 
DeCusatis06]. Note that the optical power budget is defined as the difference between the transmitted power (in $\mathrm{dB}$ ) and the receiver sensitivity (in $\mathrm{dB}$ ).

We use the following symbols:

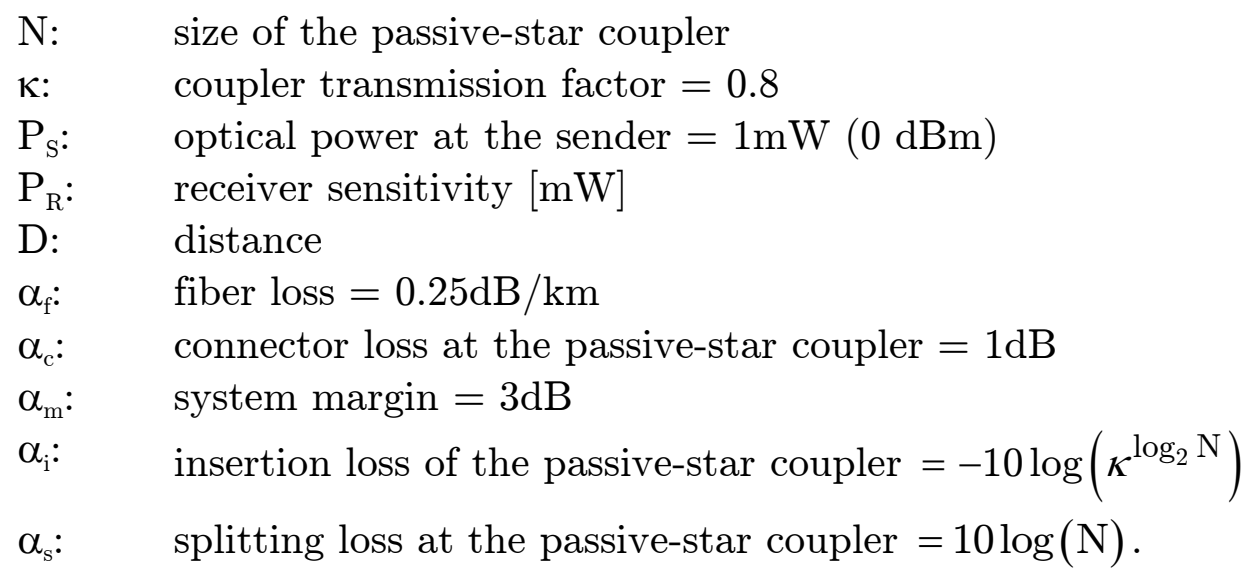

The optical power budget of the passive star coupler network in $\mathrm{dB}$ can be calculated by the following formula:

$$
\mathrm{D} \cdot \alpha_{\mathrm{f}}+\alpha_{\mathrm{i}}+\alpha_{\mathrm{c}}+\alpha_{\mathrm{s}}+\alpha_{\mathrm{m}}=10 \log \left(\frac{\mathrm{P}_{\mathrm{s}}}{\mathrm{P}_{\mathrm{r}}}\right)
$$

The maximum achievable distance between the OLT and the ONU can be calculated by converting equation (2.3) to the following relationship:

$$
\mathrm{D}_{\max }=\frac{1}{\alpha_{\mathrm{f}}}\left[10 \log \left(\frac{\mathrm{P}_{\mathrm{s}}}{\mathrm{P}_{\mathrm{r}}}\right)-\alpha_{\mathrm{i}}-\alpha_{\mathrm{c}}-\alpha_{\mathrm{s}}-\alpha_{\mathrm{m}}\right]
$$

The resulted plot for receiver sensitivities between $-15 \mathrm{dBm}$ and $-30 \mathrm{dBm}$ can be seen in Figure 2.7. The maximum distance between the network nodes mainly depends on the transmitted output power $\mathrm{P}_{S}$, the receiver sensitivity $\mathrm{P}_{\mathrm{R}}$ and the size of the passive-star coupler $\left(\alpha_{i}\right.$ depends on the size $\mathrm{N}$ and $\kappa$ of the passive-star coupler according to the symbol definition above). 


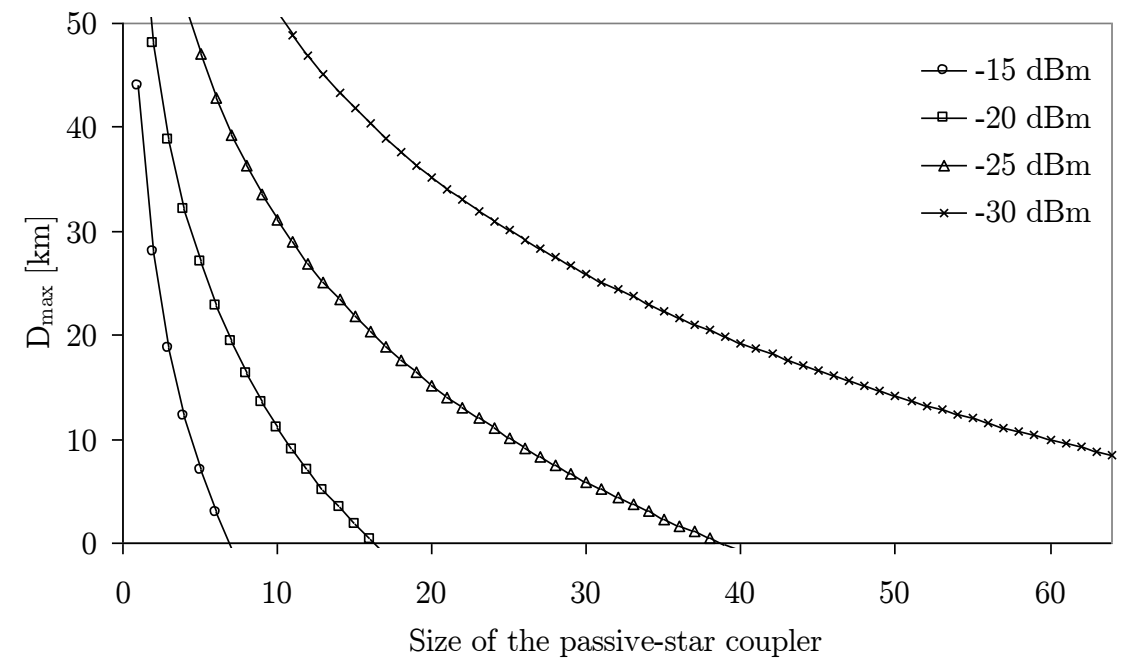

Figure 2.7: Maximum achievable distance between the OLT and the ONU with respect to the size of the passive-star coupler with receiver sensitivities between $-15 \mathrm{dBm}$ and $-30 \mathrm{dBm} . \mathrm{P}_{\mathrm{S}}=1 \mathrm{~mW}, \alpha_{\mathrm{f}}=0.25 \mathrm{~dB} / \mathrm{km}$, $\alpha_{c}=1 \mathrm{~dB}, \alpha_{\mathrm{m}}=3 \mathrm{~dB}$ and $\kappa=0.8$.

The power at each output of the passive star coupler is given by

$$
\mathrm{P}_{\mathrm{R}}=\frac{\mathrm{P}_{\mathrm{S}} \cdot \kappa^{\log _{2} \mathrm{~N}}}{\mathrm{~N}} .
$$

When the network is operated at the maximum bit rate, then the transmitted power $\mathrm{P}_{\mathrm{S}}$ will be equal to the receiver sensitivity

$$
\mathrm{P}_{\mathrm{R}}=\mathrm{hfn}_{\mathrm{p}} \mathrm{B}_{0}
$$

where $\mathrm{h}$ is the Planck's constant, $\mathrm{f}$ is the optical frequency, $\mathrm{n}_{\mathrm{p}}$ is the receiver sensitivity in photons per bit and $B_{0}$ is the channel bit rate. Insert equation (2.6) in (2.5) and convert it to the maximum network capacity derive to

$$
\mathrm{B}_{0} \cdot \mathrm{N}=\frac{\mathrm{P}_{\mathrm{S}} \cdot \kappa^{\log _{2} \mathrm{~N}}}{\mathrm{hfn}_{\mathrm{p}}}
$$

If we calculate the maximum network capacity with the assumptions of $\mathrm{P}_{\mathrm{S}}=1 \mathrm{~mW}$, $\mathrm{n}_{\mathrm{p}}=100$ photons/bit at a wavelength of $1500 \mathrm{~nm}$, this leads to a maximum network capacity in the region of $10 \mathrm{Tbit} / \mathrm{s}$. This value is valid for ideal receivers, where the performance is limited only by the constant $n_{p}$. In more realistic receivers, electronic noise and other factors further restrict the maximum network capacity which is very difficult to calculate. Therefore, [Brackett90] plotted receiver sensitivities for different direct and coherent detection receivers. The resulting curves are in the Tbit/s region and therefore out of the scope in access networks. 
The third limiting factor is the optical spectrum access. Commonly, the entire low-loss region of the optical fiber ranges from approximately $1200 \mathrm{~nm}$ to $1600 \mathrm{~nm}$. This region can be technically used for optical communication. In the case of wavelength division multiplexing, the limited channel spacing (Figure 2.8) is another additional limitation on the network capacity.

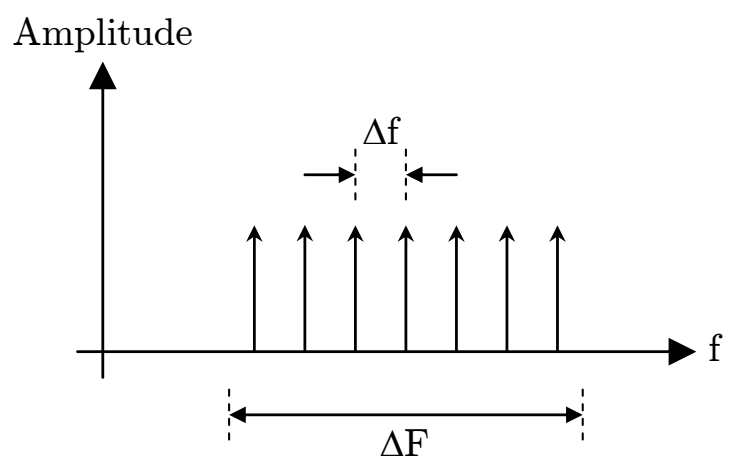

Figure 2.8: Accessible optical spectrum $\Delta \mathrm{F}$ of the optical fiber and channel spacing $\Delta \mathrm{f}$.

In practice, channel spacings are standardized in dense WDM (DWDM) systems and coarse WDM (CWDM) systems (see appendix in chapter 11). Typical channel spacings in DWDM systems are $200 \mathrm{GHz}, 100 \mathrm{GHz}$, and $50 \mathrm{GHz}$ corresponding to $1.6 \mathrm{~nm}, 0.8 \mathrm{~nm}$ and $0.4 \mathrm{~nm}$ respectively, with respect to the $1500 \mathrm{~nm}$ transmission window. In the case of CWDM systems, channel spacings of $20 \mathrm{~nm}$ are typical.

The channel spacing $\Delta \mathrm{f}$ is equal to $\mathrm{k}(\mathrm{k} \in \mathrm{N})$ times the channel bit rate $\mathrm{B}_{0}$

$$
\Delta \mathrm{f}=\mathrm{k} \cdot \mathrm{B}_{0}
$$

It has been shown that a minimum value for $\mathrm{k}$ of 6 is required to minimize the channel crosstalk [Brackett90]. Generally, the total accessible bandwidth $\Delta \mathrm{F}$ is given by

$$
\Delta \mathrm{F}=\mathrm{N}_{\omega} \cdot \Delta \mathrm{f}
$$

where $\mathrm{N}_{\omega}$ is the number of optical channels. Summing equation (2.8) and (2.9) leads to

$$
\mathrm{B}_{0} \cdot \mathrm{N}_{\omega}=\Delta \mathrm{F} / \mathrm{k}, \mathrm{k} \in \mathbb{N}
$$

In equation (2.10), the maximum network capacity $\mathrm{B}_{0} \cdot \mathrm{N}_{\omega}$ is limited by the accessible optical spectrum $\Delta \mathrm{F}$. In Figure 2.6, maximum network capacity limited by the spectrum access is depicted for $\mathrm{k}=6$ and $\Delta \mathrm{F}$ between $5 \mathrm{~nm}$ and $20 \mathrm{~nm}$.

Note, we must differentiate between the bandwidth of such a network and the throughput the network would reach in real operation. The throughput depends on the real traffic, the algorithm which controls the traffic flow, the network architecture and other factors and would therefore be very hard to estimate. Most of this is beyond the scope of this work. 


\section{Bus topology}

To realize an optical bus topology network, the number of 3-dB couplers increases with each additional node with the number of two (Figure 2.9).

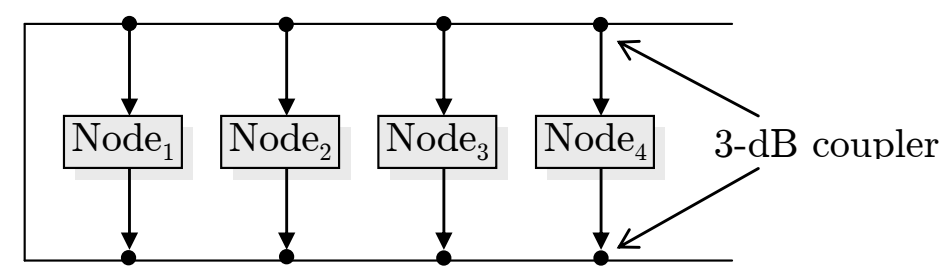

Figure 2.9: Bus topology realized with passive-star couplers.

In the worst case scenario, a single signal must be passed through $2(\mathrm{n}-1) 3$-dB couplers. This plays a significant role in determining the link budget performance of this topology.

The maximum insertion loss appears if all 2(n-1) star couplers are used for a communication procedure and can be calculated by the following equation

$$
\alpha_{\mathrm{i} \max }=(2(\mathrm{n}-1)) 10 \log \left(\kappa^{\log _{2} \mathrm{~N}}\right) .
$$

Similarly, the splitting loss increases from psc to psc. Thus, the maximum splitting loss appears if all 2(n-1) star couplers are used and can be calculated by

$$
\alpha_{\mathrm{s} \max }=(2(\mathrm{n}-1)) 10 \log (\mathrm{N}) .
$$

In almost the same manner as in equation (2.4), we can calculate the maximum achievable distance in the case of a bus topology as follows

$$
\mathrm{D}_{\max }=\frac{1}{\alpha_{\mathrm{f}}}\left[10 \log \left(\frac{\mathrm{P}_{\mathrm{S}}}{\mathrm{P}_{\mathrm{r}}}\right)-\alpha_{\mathrm{imax}}-\alpha_{\mathrm{c}}-\alpha_{\mathrm{s} \max }-\alpha_{\mathrm{m}}\right] .
$$

The calculated maximum distance with the parameters $\kappa=0.8, \mathrm{P}_{\mathrm{S}}=1 \mathrm{~mW}$, $\alpha_{\mathrm{f}}=0.25 \mathrm{~dB} / \mathrm{km}, \alpha_{\mathrm{c}}=1 \mathrm{~dB}, \alpha_{\mathrm{m}}=3 \mathrm{~dB}$ for different receiver sensitivities can be seen in Figure 2.10. The rapid decline of the maximum accessible distance with respect to the number of nodes shows us the small number of possible nodes. 


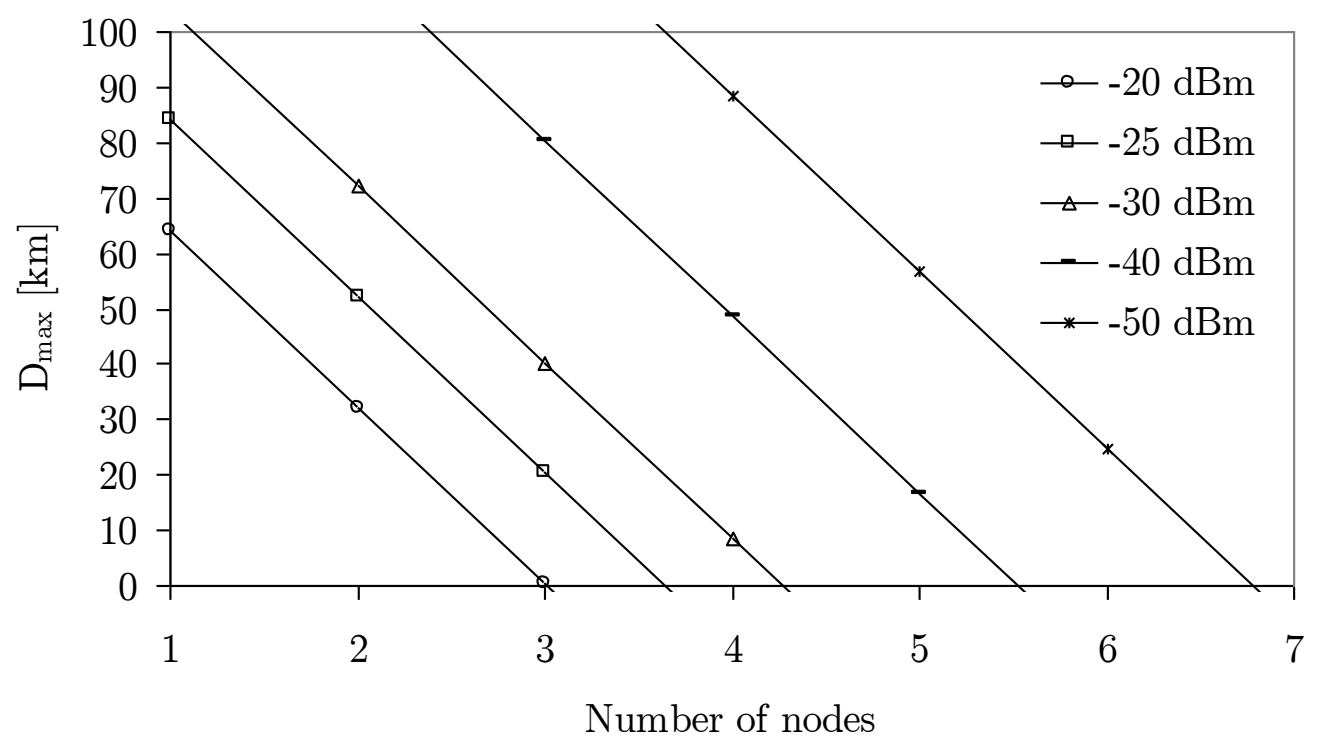

Figure 2.10: Maximum achievable distance between the OLT and the ONU with respect to the number of nodes for different receiver sensitivities. Maximum achievable distance of an optical network with bus topology with respect to the number of nodes. Due to the power budget limit, only a small number of nodes can be driven.

As a consequence, due to the poor power budget, the bus topology is not a good choice to set-up optical access networks. 


\subsubsection{Standardization}

This subsection shortly describes three important TDM PON standards. Due to the highly comprehensive standards, only the main characteristics are stated.

One of the first papers describing PONs was published by British Telecom researchers in 1988 [Stern88]. Down to the present day, several alternative architectures for TDM PON-based access networks have been standardized [Prat02]. The main difference today is the choice of the bearer protocol [Sivalingam05, p. 51 et seq.]. Currently, three different standardized specifications exist:

- ATM-based PON (APON) or broadband PON (BPON)

- Generic frame based PON (GPON)

- Ethernet based PON (EPON)

The following paragraphs give a brief overview of these standardized architectures.

\section{A/BPON}

ATM PON was formed by the Full Service Access Network (FSAN) initiative [FSAN] which is an incorporation of major network operators and many equipment vendors. The goal was to specify a specification that uses ATM as its layer-2 protocol [Killat96, Dixit03, p. 216 et seq.]. Later, the name APON was replaced by broadband PON (BPON) which symbolizes the support of Ethernet services, video services and virtual private line services [Gillespie01, Green06]. Since 1997, the ITU-T was published a series of A/B-PON-related recommendations (see chapter 11 for a list of related standards) [Nakanishi02]. APONs are mainly used in North America [Kettler00, Lin06].

Figure 2.11 shows a typical APON network. In the downstream direction, the transmission takes place with a continuous ATM data-stream at a bitrate of $155 \mathrm{Mbit} / \mathrm{s}$ or $622 \mathrm{Mbit} / \mathrm{s}$. In the upstream direction, the transmission takes place with bursts of ATM cells at a bitrate of $155 \mathrm{Mbit} / \mathrm{s}$.

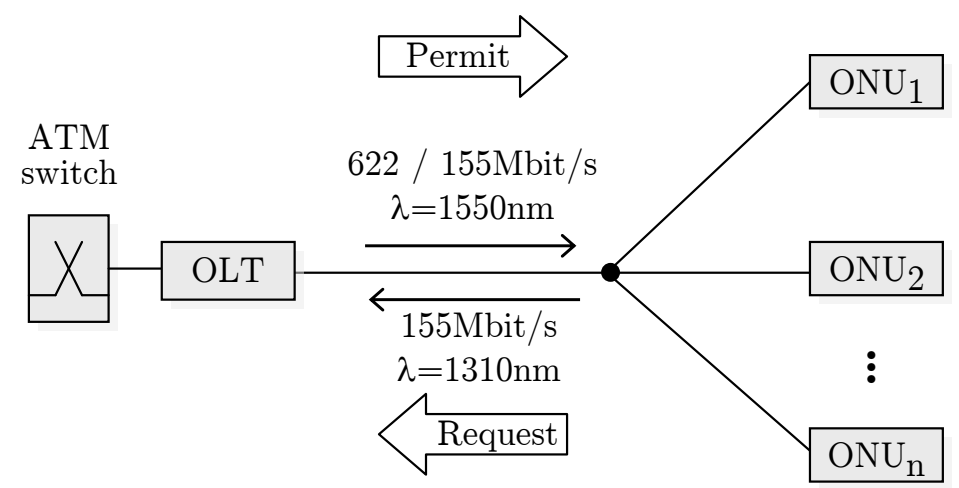

Figure 2.11: Structure of a typical APON network according to ITU-T G.983. In the upstream direction, a request-permit mechanism as an APON medium access control (MAC) protocol is used to avoid collisions. 
Due to the power budget limits, distances are limited to $10 \mathrm{~km}$ and $20 \mathrm{~km}$ with a splitting ratio of 64 and 32 respectively as a standard. For a full-duplex transmission, two distinct wavelengths must be used $(1550 \mathrm{~nm}$ for the downstream and $1310 \mathrm{~nm}$ for the upstream direction).

In the downstream direction, bursts of 56 cells contain two physical layer operation and maintenance (PLOAM) cells (Figure 2.12); one PLOAM cell at the beginning and one PLOAM cell in the middle of the burst. Each PLOAM cell contains upstream permission for each 53 upstream cells per burst and, furthermore, data for operation, administration, maintenance and provisioning (OAMP).

An upstream transmission consists of 53 ATM cells with a 3-byte header. The 3-byte header is used as a guard time between upstream slots and to resynchronize the phase locked loop (PLL) at the OLT receiver. Between the 53 upstream slots, a time slot called multi-burst slot (MBS) is used to transport the ONUs bandwidth-requests.

APON uses a request-permit procedure to prevent collisions in the upstream direction and to maximize the utilization of the medium (Figure 2.11) [Moon01]. For each ATM cell which will be sent in the upstream direction, a bandwidth-request must be sent to the OLT. The OLT then gives the permission to the basis of the currently unused bandwidth and the considered connection.

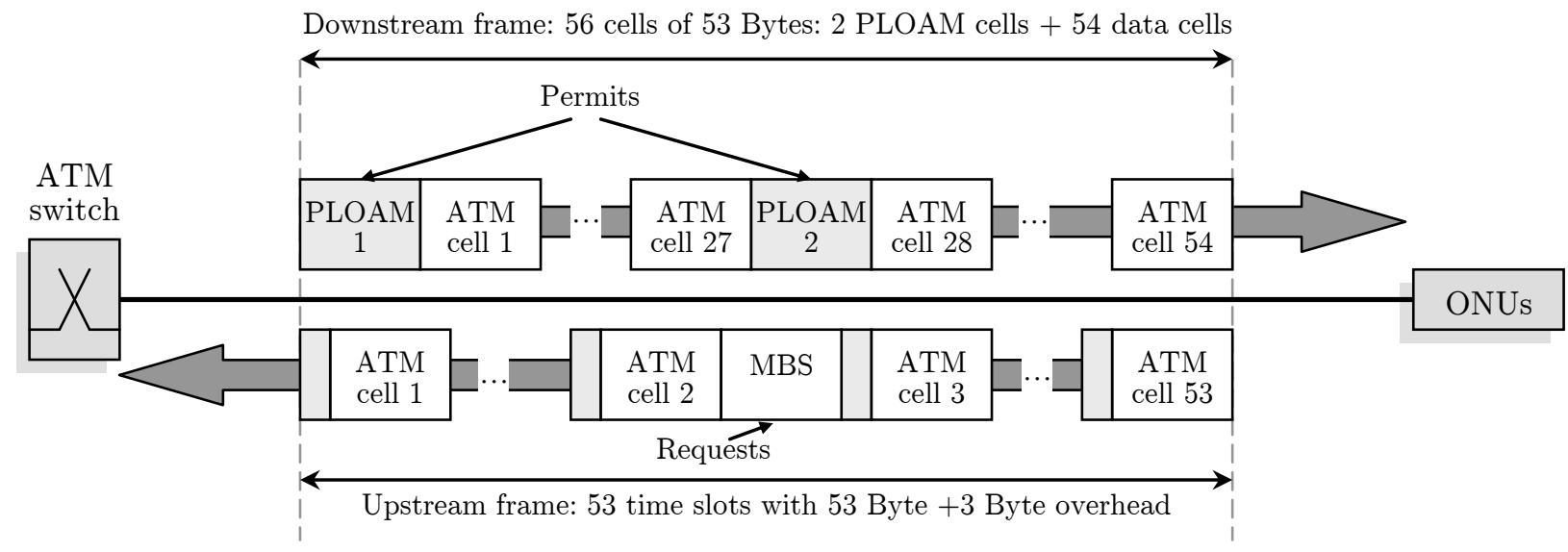

Figure 2.12: Format of the APON downstream and upstream frame according to ITU-T G.983.

\section{GPON}

Because of the ever growing traffic volume, the FSAN group [FSAN] strove to standardize a new PON (Generalized PON) network with bitrates higher than 1Gbit/s and improved efficiency for data traffic [Green06]. To overcome the inefficiency in the ATM based PON, the generic framing procedure (GFP) [ITU-T G.7041] can be used, while allowing a combination of ATM cells and variable-size frames. GPON is standardized in the ITU-T standard G.984.1 [ITU-T G984.1], G.984.2 [ITU-T G984.2] and G.984.3 [ITU-T G984.3].

In summary, GPON includes full service support like voice (POTS, ISDN), Ethernet (10/100 BaseT), ATM, leased lines and more. In principle, GPON supports various bitrate options. Downstream direction bitrates of 1.2 and $2.4 \mathrm{Gbit} / \mathrm{s}$ and upstream direction bitrates of $155 \mathrm{Mbit} / \mathrm{s}, 622 \mathrm{Mbit} / \mathrm{s}, 1.2 \mathrm{Gbit} / \mathrm{s}$ and $2.4 \mathrm{Gbit} / \mathrm{s}$ are standardized.

On the basis of the power budget limit and dispersion limitations, the distance between the OLT and the ONU is standardized and cannot exceed the distance $10 \mathrm{~km}$ or $20 \mathrm{~km}$ 
(in dependence of the bitrate used). The transmission convergence (TC) layer permits split ratios up to $1: 128$.

The GPON standard defines different security mechanisms which can be used for automatic or manual switching. The primary goal of the security mechanism is to make the optical distribution network (ODN) secure. Automatic switching is triggered by the loss of signal or loss of frame to go below a predefined bit-error-rate (BER). Manual switching is triggered by administrative events (e.g. rerouting over a further fiber).

The biggest advantage of the GPON is its multi-service support, especially a large amount of TDM services which can be supported efficiently. Thus, for Fiber-to-theOffice (FTTO), GPON is the preferred PON technology [Lin06].

\section{EPON}

Ethernet PON (EPON) (also termed as Ethernet in the first mile (EFM)) is a PONbased network that carries data traffic encapsulated in Ethernet frames [Dixit03, p. 229 et seq., Kramer03, Kramer05, Green06]. In most countries, EPON is the dominant PON technology for homes and small offices [Lin06]. In principle, two modes of operation are standardized. In the first one, it can be deployed over a shared medium using the carrier-sense multiple access with collision detection (CSMA/CD) protocol. In the second one, stations can be connected via full duplex point-to-point links. It uses a standard $8 \mathrm{~b} / 10 \mathrm{~b}$ line coding and operates at standard Ethernet speed.

In the downstream direction, Ethernet frames transmitted by the OLT pass through a 1:N passive star coupler and therefore reach each ONU. $\mathrm{N}$ is standardized between 4 and 64 . In the downstream direction, packets are broadcast by the OLT and extracted by their destination $\mathrm{ONU}$ based on the media-access control (MAC) address (Figure $2.13)$.

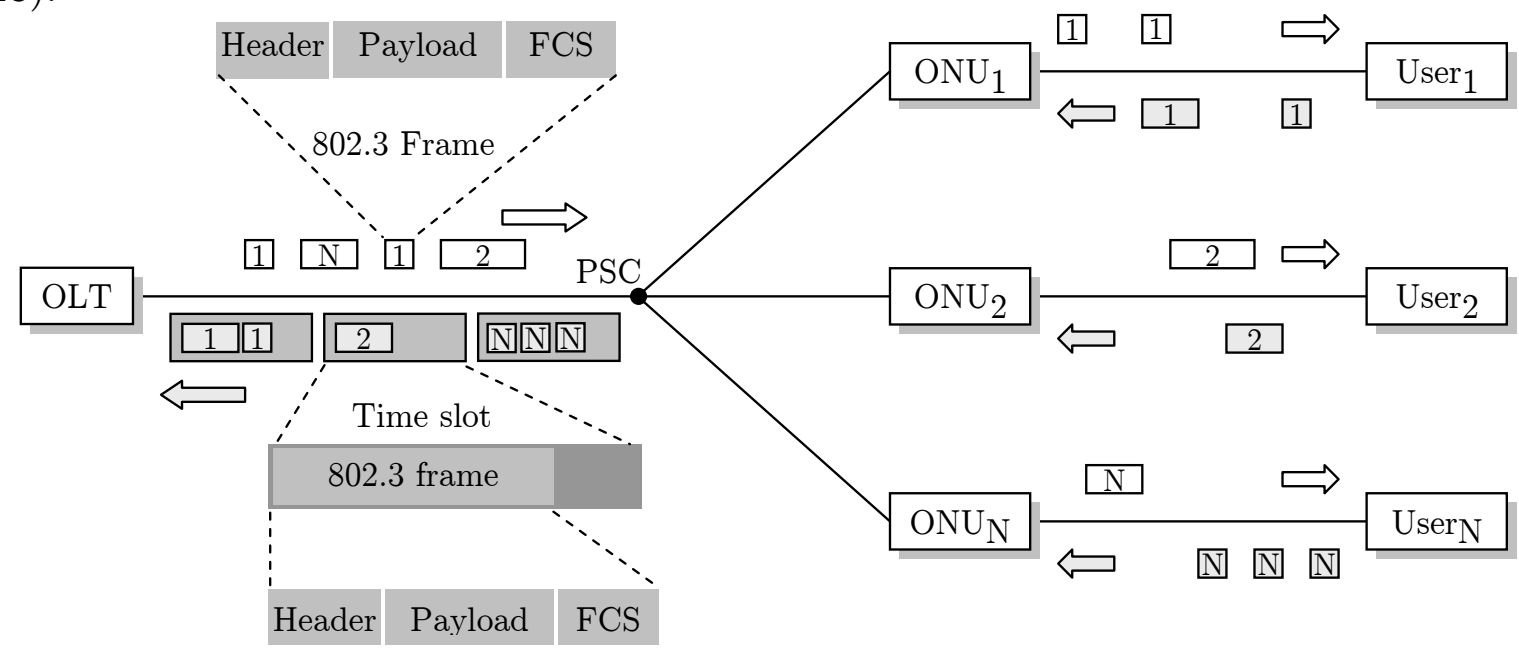

Figure 2.13: Downstream and upstream traffic in an EPON network. In the upstream direction, TDM is needed to prevent collisions.

In the upstream direction, due to the directional properties of a passive star coupler, data frames from any ONU will reach only the OLT, and not other ONUs. If data frames from different ONUs are transmitted simultaneously, they may still collide. Thus, in the upstream direction, the ONUs needs to employ some arbitration mechanism to avoid data collisions and share the fiber-channel capacity fairly. Figure 2.13 illustrates an upstream non-contention-based time-shared scheme. All ONUs are 
synchronized to a common time reference and each $\mathrm{ONU}$ is allocated to a time slot. An ONU should buffer frames received from a user until its timeslot arrives.

The possible time slot allocation schemes could range from a statistic allocation TDMA (time division multiple access) to a dynamically adapting scheme based on an instantaneous queue size in every ONU (statistical multiplexing scheme). More allocation schemes are possible, including schemes utilizing notions of traffic priority, quality of service (QoS), service-level-agreements (SLAs), oversubscription, and so on [Kramer01].

\subsubsection{Overhead impact}

One significant factor for network providers is the efficiency of the network technology. The efficiency is a performance parameter and is influenced by the overhead bytes. It is important for network providers to account for a maximum of transferred bits. These clearable bits are called revenue bits. The undesirable overhead bytes degradate the revenue bits. The efficiency is given by

$$
\eta_{\mathrm{var}}=\frac{\mathrm{X}}{\mathrm{X}+\mathrm{OH}}
$$

whereas $\mathrm{X}$ is the payload and $\mathrm{OH}$ the overhead, both in bytes or bits. It is commonly known that ATM has a constant cell-size of 53 bytes [Siegmund97, Haßlinger99]. Therefore, the number of transmitted cells depends on the cell-size and can be calculated by

$$
\mathrm{N}=\left|\frac{\mathrm{X}}{\mathrm{L}}\right|
$$

at which L is the constant ATM cell-size of 53 bytes. Substituting equation (2.15) in (2.14) leads to

$$
\eta_{\text {const }}=\frac{\mathrm{X}}{\left|\frac{\mathrm{X}}{\mathrm{L}}\right| \cdot \mathrm{L}+\mathrm{OH}}
$$

and represents the efficiency in dependence on the number of transmitted cells. In summary we can say equation (2.14) represents the efficiency for protocols with variable payload size, and equation (2.16) can be used for protocols with fixed payload sizes.

Due to the different ATM Adaptation Layer (AAL) in ATM, overhead bytes differ from 5 bytes (AAL 5) and 9 bytes (AAL 3/4) [Siegmund97, Haßlinger99, Gillespie01]. As we can see in Figure 2.14, the best possible efficiency for ATM is $90.6 \%$ with AAL 5 and $83 \%$ with AAL $3 / 4$. It can be seen that carrying variable-length IP packets over ATM leads to higher overhead, even though we know that IP packets are the predominant component of the internet traffic.

Another shortcoming of ATM is the fact that a corrupted or dropped ATM cell will invalidate the entire IP datagram. If this scenario occurs, the remaining ATM cells 
carrying the portions of the same IP datagram will propagate further. In this case, network resources will be wasted unnecessarily [Siegmund97, Haßlinger99].

In the case of Ethernet, encapsulation overhead consists of adding an 8 byte frame preamble, a 14Byte Ethernet header, and a 4Byte frame check sum (FCS) field. In addition, a 12Byte minimum interframe gap (IFG) is used between two adjacent frames. Therefore, the total overhead per single frame is 38Byte and constant (Figure 2.14).

Due to the line coding in Ethernet, the physical bit rate is greater than the nominal one by a factor of 1.25 . Thus, the efficiency is given by

$$
\eta_{\text {phys }}=\frac{\mathrm{X}}{(\mathrm{X}+\mathrm{OH}) \cdot 1.25}
$$

and is illustrated in Figure 2.14 (Ethernet physical). In this case we realize a maximum of the efficiency at $78 \%$.

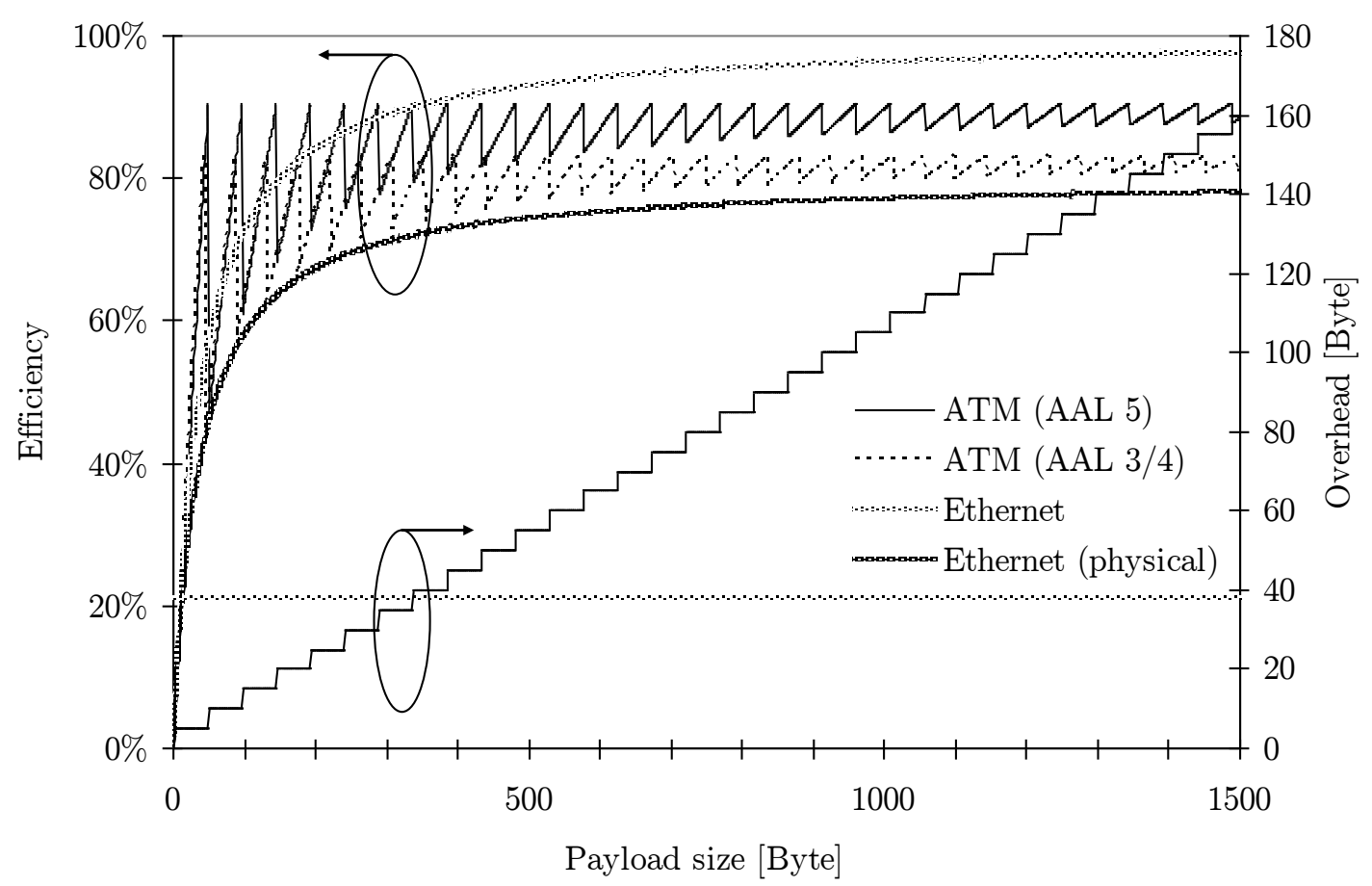

Figure 2.14: Comparison of the efficiency of ATM and Ethernet in dependence of the payload size.

In a real environment, the average value of the encapsulation overhead depends on the distribution of the payload size. [Sala01] reported these datagram size distributions obtained by an access network (Table 2.1).

These distributions have three main spikes in downstream and upstream direction corresponding to 40 byte, 576 byte, and 1500 byte payload size. As we can see in Table 2.1, these three spikes are in summary $80 \%$ of the downstream volume and $72 \%$ of the upstream volume. The remaining $20 \%$ and $28 \%$ of the downstream and upstream directions are respectively distributed over the remaining payload

\begin{tabular}{rrr}
\hline Payload & Downstream & Upstream \\
\hline 40 & $34 \%$ & $46 \%$ \\
576 & $18 \%$ & $5 \%$ \\
1500 & $28 \%$ & $21 \%$ \\
\hline & $80 \%$ & $72 \%$
\end{tabular}

Table 2.1: Payload size distributions obtained by an access network [Sala01]. size and are unaccounted for further calculations. The calculated efficiency in Figure 
2.14 can now be weighted with the distribution in Table 2.1 and can be expressed as follows

$$
\eta=\sum_{\mathrm{n}=1}^{\max \cdot \text { payload }} \eta(\text { payload }) \cdot \mathrm{X}(\text { payload })
$$

where $\eta$ (payload) is the network efficiency of a specific technology, and $\mathrm{X}$ (payload) is the standardized payload distribution. The result is a realistic efficiency of different network technologies and is presented in Figure 2.15.

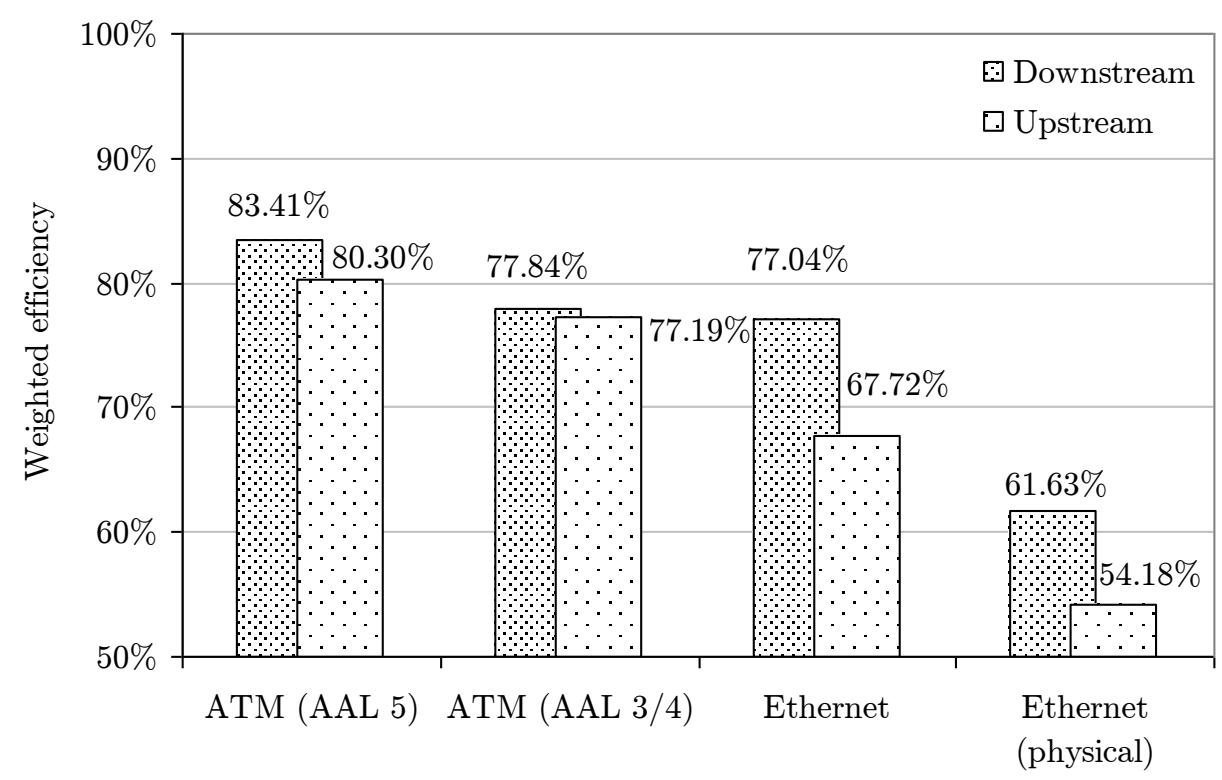

Figure 2.15: Weighted efficiency of different network technologies. The weighting function is the payload distribution obtained by an access network [Sala01].

Due to the payload distribution peak at 40 bytes and the almost optimal ATM cell payload size of 48 byte (AAL 5) and 44 byte (AAL 3/4), the efficiency of ATM is significantly better than the efficiency of Ethernet. It is obvious that the efficiency for ATM is in the range between approximately $83 \%$ and $77 \%$, whereas the real or physical efficiency of Ethernet is roughly in the range between $62 \%$ and $54 \%$. In the literature, many efficiency comparisons of different network technologies are presented [Kramer05]. In most cases these comparisons use the nominal bitrate of Ethernet which leads to a significantly better efficiency for Ethernet.

In the next section, the transition from time division mutliplexed to wavelength division multiplexed optical networks is performed. 


\subsection{WDM PON}

While a TDM PON uses a broadcast structure in which all users receive copies of the same optical signal, it has been realized that wavelength-division multiplexing (WDM) had certain advantages: increased capacity, security, privacy, upgradability [Iannone98, Krishna00, Gumaste02]. Due to the collision-free nature of a WDM PON, it offers the nfold bandwidth compared with TDM PONs implemented with a passive star coupler. This fact implies the evolution of FTTH solutions illustrated in Figure 2.16. It should be indicated that B-ISDN and xDSL are not pure FTTH solutions. But it illustrates the ever increasing fiber penetration and the fact that fibers are moving closer to the user.

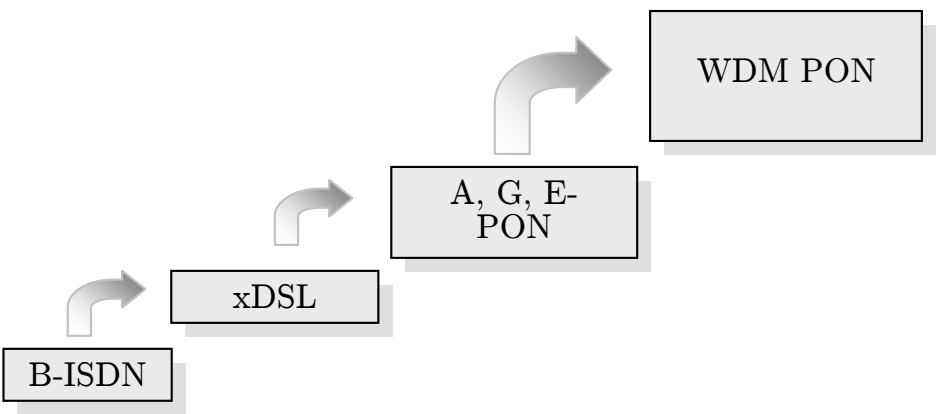

Figure 2.16: Evolution of FTTH solutions.

In the following subsections, the physical functionality of the components required to build a WDM PON is described. The upstream and downstream transmissions in a WDM PON differ from a TDM PON and are therefore parts of this section. Standardizations that affect WDM PONs form the last section.

\subsubsection{Technological Background}

The passive-star coupler (described in subsection 2.3.1) represents a broadcast-andselect component in which each optical input signal is equally distributed to all output ports. This reality leads to optical power splitting losses and can be avoided by using wavelength router (WR). In principle, there are several possible implementations of WR depending on the physical concept [Mestdagh95, Wan00, Agrawal02, Keiser03]. The used optical filtering technique is crucial for the realization of a WR which leads to the classification of different WR realizations

- Arrayed Waveguide Grating (AWG)

- Grating based (GB)

- Thin-Film Filter (TFF)

- Fiber Bragg Grating (FBG)

- Mach-Zehnder Interferometer (MZI)

- Fabry-Perot Interferometer (FPI).

The preferred embodiment of the wavelength router is the arrayed-waveguide-grating multiplexer (AWGM), also known as wavelength grating router (WGR or simply WR) or an optical phased array (PHASAR). Detailed information of the other listed 
embodiments of the WR can be found in the literature [Kashima95, Othonos99, Chang03, p. 935 et seq., Palais04, Lin04, Goodman05, Pedrotti07]. Each wavelength can be simultaneously used on all input ports without channel collision, thus enabling wavelength reuse within the network [Barry93, Glance94, Smit96, Milorad04, Lin04, Sivalingam05].

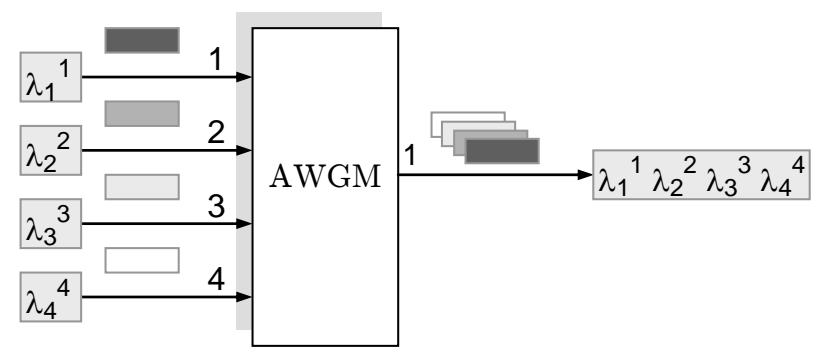

(a) Multiplexer

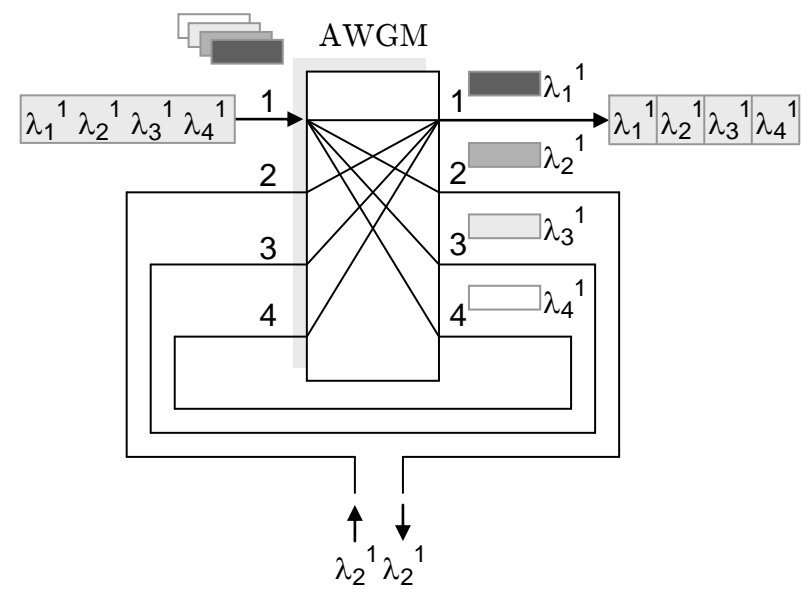

(c) Add-drop multiplexer

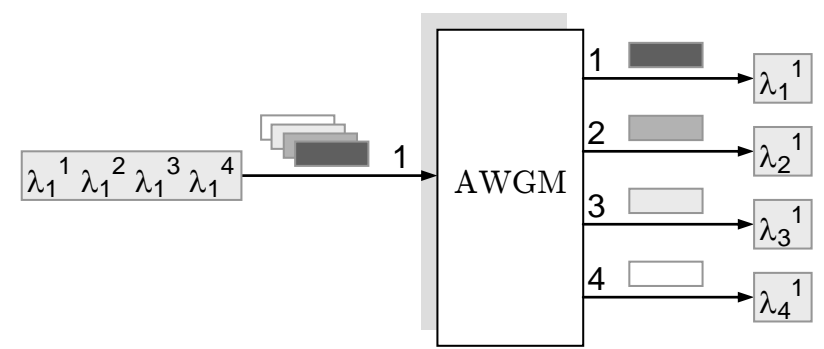

(b) Demultiplexer

Figure 2.17: Schematic illustration of the functionality of an AWGM device. Potential applications are the wavelength multiplexer (a), demultiplexer (b), the add-drop multiplexer (c) and the static wavelength router $(\mathrm{d})$. In the case of the static wavelength router, 4 wavelengths per input port were cyclically routed to the 4 output ports without collisions. Therefore, wavelength reuse is feasible.

Several applications can be imagined with such an AWGM device. The AWGM is mostly used as a wavelength mutliplexer (Figure 2.17 (a)) and a wavelength demultiplexer (Figure 2.17 (b)) [Takahashi95]. Both applications allow for setting up WDM networks. If we join the wavelength multiplexer and demultiplexer, we create a new component called the static wavelength router (see Figure $2.17(\mathrm{~d})$ ). The fourth application is add-drop multiplexing which is essential to set up optical WDM rings [Takahashi95].

As shown in Figure 2.17 (d), all available wavelengths at input port 1 are routed to port numbered 1, 2, 3 and 4 without having collisions with other wavelengths of the other input ports 2,3 , and 4 . The wavelengths of the other input ports 2,3 , and 4 are routed similarly in a cyclic way.

Such a device is a passive router since its use does not involve an active element requiring electrical power. Note that AWGMs are also used for realizing optical WDM 
components such as WDM multiplexers/demultiplexers, optical add/drop multiplexers (OADM), optical cross connects (OXC), and other important optical wavelengthselective devices. Convenient properties of the AWGM is the support of a high number of WDM channels (e.g. 80 x 80 AWGMs are currently commercially available [Photeon05]) and its favor channel selection characteristics.

Compared to a passive-star coupler, in which a given wavelength may only be used on a single input port, the AWGM with $\mathrm{M}$ input and $\mathrm{M}$ output ports is capable of routing a maximum of $\mathrm{M}^{2}$ connections, as opposed to a maximum of $\mathrm{M}$ connections in the passivestar coupler. Because the AWGM is an integrated device, it can be fabricated at low cost. The disadvantage of the AWGM is the fixed routing matrix which cannot be dynamically reconfigured. Despite its static nature, a AGWM router has many applications in WDM networks as stated before.

A closer examination of the use of an AWGM device with M input and M output ports in a WDM network, where each port carries $\mathrm{n}$ different wavelengths, allows us to show the functionality. Due to the physical functionality of this waveguide routing device described above, the $\mathrm{n}$ wavelengths arriving at an input port are routed to different output ports corresponding to a fixed routing matrix. Figure 2.17(c) presents the principle of operation for a 4 x 4 AWGM router.

As a result of the non-blocking property of the AWGM router, optical signals on the same wavelength are routed from different input ports to different output ports without interfering within the device. The wavelengths are routed as virtual channels $\lambda_{i}^{k}$, where the subscripted index $\mathrm{i}$ denotes the physical wavelength and the superscripted index $\mathrm{k}$ represents the input port number.

For a better understanding of the important AWGM device, the physical functionality is explained below.

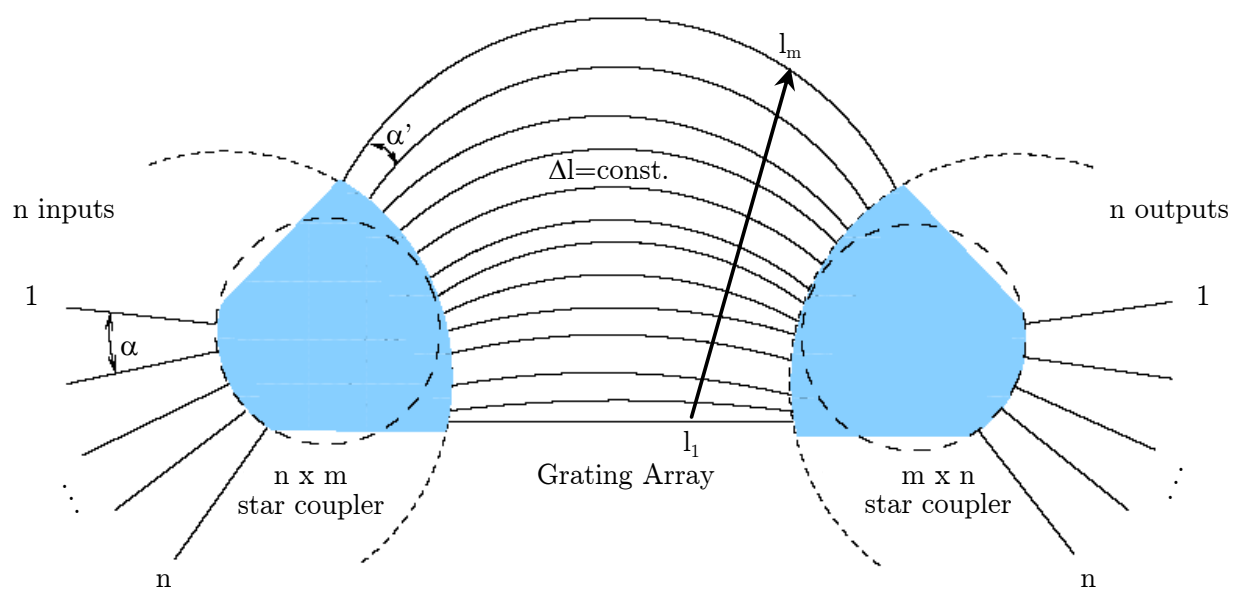

Figure 2.18: A schematic structure of an AWGM device.

As shown in Figure 2.18, the AWGM is made up of two regular passive-star couplers which are interconnected by a grating array [Kaminow02a, p. 420 et seq., Goodman05, p. 420 et seq.]. The number of inputs and outputs of the AWGM are denoted by $\mathrm{n}$ and the number of arrayed waveguides are $\mathrm{m}$. Thus, the passive-star couplers at the input 
and the output is $\mathrm{n} \times \mathrm{m}$ and $\mathrm{m} \times \mathrm{n}$ in size, respectively. The grating array consists of $\mathrm{m}$ waveguides, with the lengths $l_{1}, l_{2}, \ldots, l_{m}$ where $l_{1}<l_{2}<\ldots<l_{m}$. The length difference between the consecutive arrayed waveguides is constant over all waveguides and denoted by $\Delta \mathrm{l}$.

Now we will determine which wavelength will be transmitted from a given input port to a given output port. The first coupler splits the incoming signal into $\mathrm{m}$ parts. The relative phases of these parts are appointed by the different distances traveled in the coupler from the input waveguides to the arrayed waveguides. The differences in the distances traveled between input waveguide $\mathrm{i}$ and arrayed waveguide $\mathrm{j}$ are denoted by $\mathrm{d}_{\mathrm{in}}{ }^{\mathrm{ij}}$. The length difference between arrayed waveguide $\mathrm{j}$ has a path larger than arrayed waveguide $\mathrm{j}-1$, denoted by $\Delta \mathrm{l}$. The signal travels through the grating array, experiencing a different phase shift in each waveguide depending on the length of the waveguides and the wavelength of the signal. The constant difference in the lengths of the waveguides creates a phase difference in adjacent waveguides of [Smit96][Ramaswami02]

$$
\Delta \Phi=\beta \cdot \Delta \mathrm{l}
$$

whereas $\beta$ is the propagation constant in the waveguide and is defined as

$$
\beta=\frac{2 \pi \mathrm{n}_{2}}{\lambda}
$$

$\mathrm{n}_{2}$ is the refractive index of the waveguide and $\lambda$ is the wavelength of the light.

Likewisely, denote the differences in the distances between arrayed waveguide $\mathrm{j}$ and output waveguide $\mathrm{k}$ by $\mathrm{d}_{\text {out }}{ }^{\mathrm{jk}}$. The relative phases of the signals from input $\mathrm{i}$ to output $\mathrm{k}$ carrying the $\mathrm{m}$ different paths between them are given by [Ramaswami02]

$$
\phi^{\mathrm{ijk}}=\frac{2 \pi}{\lambda}\left(\mathrm{n}_{1} \mathrm{~d}_{\mathrm{in}}^{\mathrm{ij}}+\mathrm{n}_{2} \mathrm{k} \Delta \mathrm{l}+\mathrm{n}_{1} \mathrm{~d}_{\text {out }}^{\mathrm{jk}}\right) \quad \mathrm{k}=1,2, \ldots, \mathrm{m}
$$

whereas $\mathrm{n}_{1}$ is the refractive index in the input and output couplers and $\mathrm{n}_{2}$ is the refractive index in the arrayed waveguides. Those wavelengths from any input port $\mathrm{i}$ for which $\phi_{\mathrm{ijk}}$ differs from a multiple of $2 \pi$ will add in phase at output $\mathrm{k}$ and we will call this constructive interference.

If the differences in the distances from the input and output couplers are designed such that

$$
\mathrm{d}_{\mathrm{in}}^{\mathrm{ij}}=\mathrm{d}_{\mathrm{in}}^{\mathrm{i}}+\mathrm{k} \delta_{\mathrm{in}}^{\mathrm{i}}
$$

and

$$
\mathrm{d}_{\text {out }}^{\mathrm{jk}}=\mathrm{d}_{\text {out }}^{\mathrm{k}}+\mathrm{k} \delta_{\text {out }}^{\mathrm{k}}
$$

then (2.21) can be rewritten as 


$$
\phi^{\mathrm{ijk}}=\frac{2 \pi}{\lambda}\left(\mathrm{n}_{1} \mathrm{~d}_{\text {in }}^{\mathrm{i}}+\mathrm{n}_{1} \mathrm{~d}_{\text {out }}^{\mathrm{j}}\right)+\frac{2 \pi}{\lambda} \mathrm{k}\left(\mathrm{n}_{1} \delta_{\text {in }}^{\mathrm{i}}+\mathrm{n}_{2} \Delta \mathrm{l}+\mathrm{n}_{1} \delta_{\text {out }}^{\mathrm{j}}\right) \quad \mathrm{k}=1,2, \ldots, \mathrm{m} .
$$

The symbol $\delta$ represents the Kronecker delta and is defined as follows

$$
\delta_{\mathrm{ij}}=\left\{\begin{array}{l}
1 \text { if } \mathrm{i}=\mathrm{j} \\
0 \text { if } \mathrm{i} \neq \mathrm{j}
\end{array} .\right.
$$

Those wavelengths $\lambda$ that attend at input port $i$ and that satisfy $\mathrm{n}_{1} \delta_{\text {in }}^{\mathrm{i}}+\mathrm{n}_{2} \Delta \mathrm{l}+\mathrm{n}_{1} \delta_{\text {out }}^{\mathrm{j}}=\mathrm{p} \lambda$ for some integer $\mathrm{p}$ add in phase at output port $\mathrm{k}$. In this case, the transmission power from a particular input port $\mathrm{i}$ to a particular output port $\mathrm{k}$ is maximized when $\Phi^{\mathrm{ijk}}$ is a multiple of $2 \pi$. Such a construction is possible with a Rowland circle construction (Figure 2.19).

Two signals of the same wavelength coming from two different input ports will not interfere with each other in the grating array because there is an additional phase difference created by the distance between the two input ports in the first star coupler. The two optical signals will be combined in the grating but will be separated out again in the second star coupler and directed to different outputs.

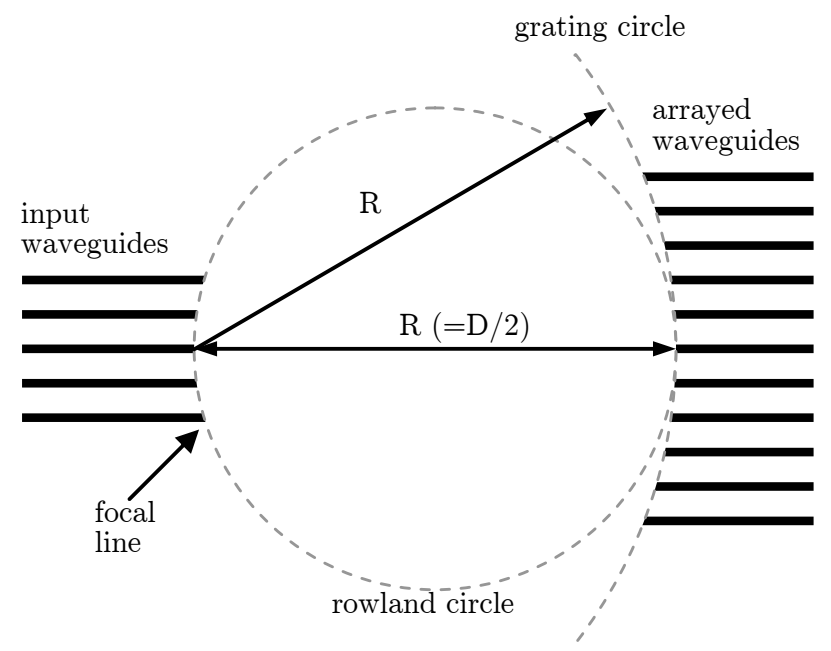

Figure 2.19: Principle operation of an AWGM device. The input waveguides are located on the arc of a circle whose diameter is equal to $\mathrm{R}$. This circle is called the Rowland circle. The arrayed waveguides are located on the arc of a circle with the radius $\mathrm{R}$, called the grating circle. The center of the grating circle is at the end of the central input waveguide. The vertical space between the arrayed waveguides is chosen to be constant [Ramaswami02].

When we use the AWGM as a demultiplexer, all wavelengths are present at the same input $i$. If the wavelengths $\lambda_{1}, \lambda_{2}, \ldots, \lambda_{n}$ in the WDM system satisfy $\mathrm{n}_{1} \delta_{\text {in }}^{\mathrm{i}}+\mathrm{n}_{2} \Delta \mathrm{l}+\mathrm{n}_{1} \delta_{\text {out }}^{\mathrm{j}}=\mathrm{p} \lambda_{\mathrm{k}}$ for some integer $\mathrm{p}$, these wavelengths are demultiplexed by the AWGM. If another wavelength $\lambda_{\mathrm{k}}{ }^{\prime}$ at the same input port $\mathrm{i}$ satisfies $\mathrm{n}_{1} \delta_{\text {in }}^{\mathrm{i}}+\mathrm{n}_{2} \Delta \mathrm{l}+\mathrm{n}_{1} \delta_{\text {out }}^{\mathrm{j}}=(\mathrm{p}+1) \lambda_{\mathrm{k}}^{\prime}$, then both $\lambda_{\mathrm{k}}$ and $\lambda_{\mathrm{k}}{ }^{\prime}$ are demultiplexed to output $\mathrm{k}$. The AWGM multiplexer/demultiplexer has a periodic frequency response and all the wavelengths must lie within one free spectral range (FSR). The FSR and its usage is explained in detail in section 3.1 .

Please note that the name AWGM and wavelength router (WR) are likewise used in this work. 


\subsubsection{Downstream Transmission}

Downstream transmission in a WDM PON requires a multi-wavelength source at the OLT and a wavelength demultiplexer which acts as a branching device [Kaminow02b, p. 485 et seq., Dutta04, p. 221 et seq.]. There are many approaches to build a multiwavelength source. It can be made from 16 discrete distributed feedback (DFB) lasers or with an integrated multiwavelength source [Iannone96, Tachikawa96, Agrawal02]. In the case of an integrated multiwavelength source, we need $U \cdot U_{\omega}=I_{1} \cdot n_{\omega}$ (=number of wavelengths in the whole WDM PON) independent electrooptical modulators to encode the various downstream channels (Figure 2.20). We also require a splitter, $\mathrm{I}_{1}$ times WDM demultiplexer and $\mathrm{I}_{1}$ times WDM multiplexer. This solution allows for sharing the costs of the multiwavelength laser and splitter by a factor of $U \cdot U_{\omega}$ (or $\mathrm{I}_{1} \cdot \mathrm{n}_{\omega}$ ) which is equal to the number of wavelengths in the whole WDM PON. The cost of the WDM demultiplexer and WDM multiplexer are shared by a factor of $\mathrm{I}_{1}$.

Central Office

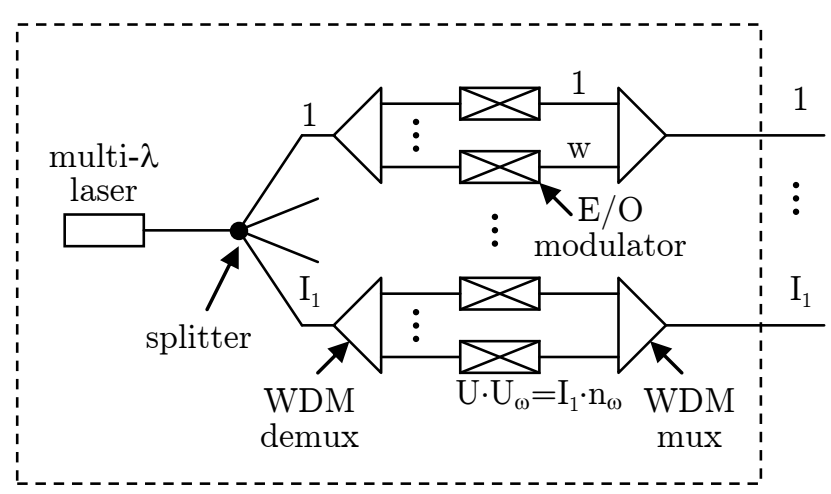

Figure 2.20: A cost-effective central office solution with the usage of a multiwavelength source.

Another significant advantage is the usage of the same optical source which acts as a carrier for each channel. It is clear that the control of the wavelength stability is more easily compared to other solutions which use $\mathrm{U} \cdot \mathrm{U}_{\omega}$ independently lasers.

As a result of the facts mentioned above, the number of used outputs at the $\mathrm{CO}\left(\mathrm{I}_{1}\right)$ should be maximized to minimize the cost.

If we operate a WDM PON under real conditions, temperature changes influence AWGMs in a way that has major implications for operation. The passbands drift with temperature at a rate of about $0.0075 \mathrm{~nm} /{ }^{\circ} \mathrm{C}$ [Wagner89]. If the AWGM is outdoors in a WDM PON network, the temperature can range from $-40^{\circ} \mathrm{C}$ to $+85^{\circ} \mathrm{C}$. The passbands are shifted by about $1 \mathrm{~nm}$. When we use a DWDM system, the channel spacing is $100 \mathrm{GHz}$ or $200 \mathrm{GHz}$ which corresponds to $0.8 \mathrm{~nm}$ or $1.6 \mathrm{~nm}$ in the third wavelength window [Kartalopoulos00]. Thus, the channel spacing of the AWGM will be in the same region as the temperature-induced shift. With these channel spacings, it will be necessary to monitor and track the wavelengths or to utilize wavelength routers with much-reduced temperature dependence.

An alternative approach to wavelength tracking is to use the set-and-forget strategy. Broaden channel spacings are required so that the wavelengths from the multiwavelength source remain within the passbands of the router throughout the entire 
temperature range. This simplifies the system by eliminating the need for wavelength tracking.

\subsubsection{Upstream Transmission}

In the upstream direction (from the ONU to the OLT), the AWGM acts as an optical multiplexer. Therefore, the OLT has a WDM receiver consisting of an optical demultiplexer followed by a receiver array.

A special issue is the optical source in the ONU. Service providers would strongly prefer all ONUs to be identical so that any ONU can be connected to any port of the router (colorless or wavelength independent ONU). It helps to avoid installation difficulties, maintenance and inventory. However, the predominant factor is that the ONU must be inexpensive. The optical source must be capable of producing a wavelength that matches the optical passband of the corresponding AWGM. It must be able to operate within the entire temperature range $\left(-40^{\circ} \mathrm{C}\right.$ to $85^{\circ} \mathrm{C}$ if the $\mathrm{ONU}$ is outdoor $)$ and must be able to operate with sufficient power (see power budget considerations in section 4.4). As with the OLT source, monitoring and tracking, the ONU source must be feasible or able to use the set-and-forget strategy as stated before. Here again the advantage of having AWGM with temperature-independent passbands is clear.

Several possible methods solve the problem and are explained in detail in the following paragraphs [Kaminow02b, p. 482 et seq.].

\section{Tunable Source}

The idea of this concept is the use of a tunable source in each ONU (see Figure 2.21) [Feldman98]. If we assume a WDM PON with a 16 port AWGM and a channel spacing of $400 \mathrm{GHz}(3.2 \mathrm{~nm})$, the total required tuning range is over $50 \mathrm{~nm}$ in the third optical window. Several solutions exist such as widely tunable lasers or arrays of single frequency lasers [Agrawal02]. These solutions are currently in the experimental stage and would be even more costly than DFB lasers. If these sources become practical, linear crosstalk at the demultiplexer in the WDM receiver will be an issue [Feldman98]. The linear crosstalk problem can be controlled, but there will be costs involved [Monroy02].

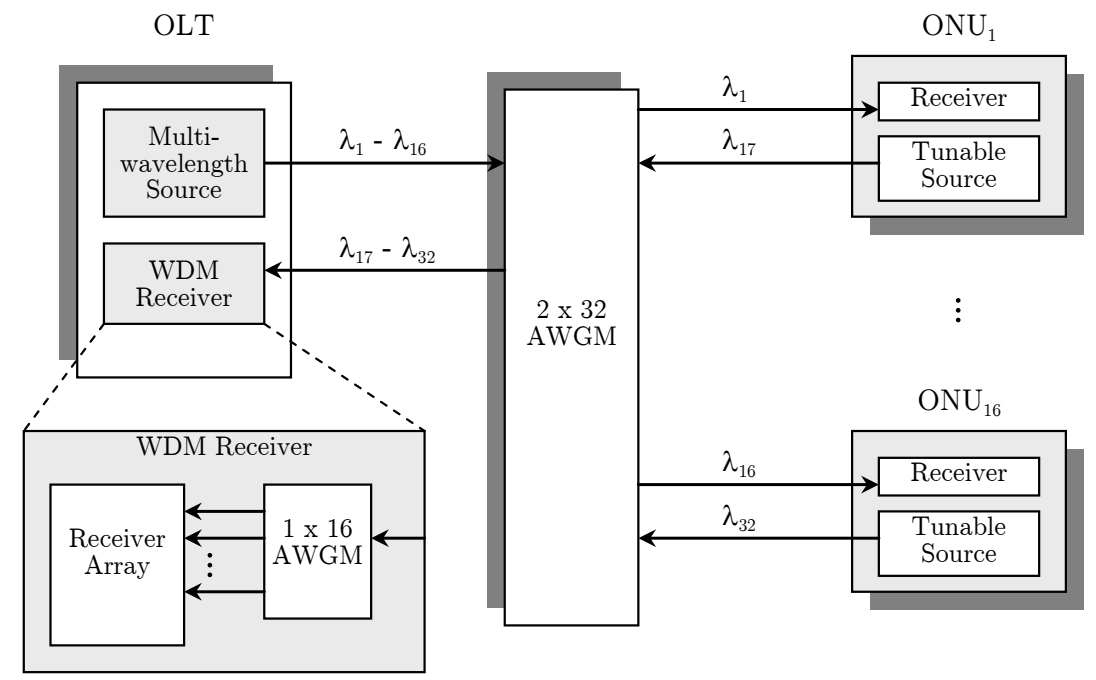

Figure 2.21: Schematic of a WDM PON with tunable source. The downstream wavelengths $\lambda_{1}-\lambda_{16}$ differ from the upstream wavelengths $\lambda_{17}-\lambda_{32}$. 


\section{Optical Loopback}

The idea of optical loopback is to avoid all the light sources in all ONUs. This option sends the downstream light through a $1 \times 2$ coupler and a portion of it through a modulator at the ONU and then loops this modulated light signal back to the OLT to avoid the transmitter at the ONU [Frigo94, Dutta04, p. 221 et seq., Suzuki04]. The architecture, shown in Figure 2.22, is also called RITENET (Remote Interrogation of Terminal Network) in the literature.

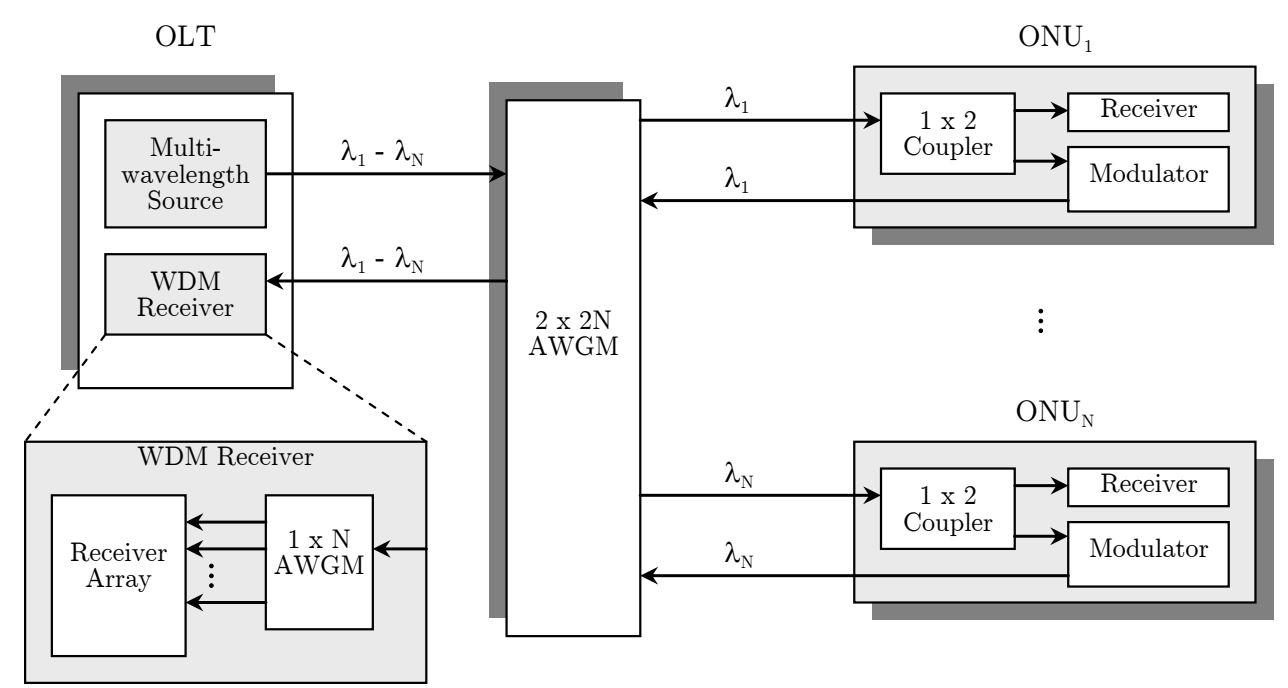

Figure 2.22: WDM PON using optical loopback. A portion of the optical downstream signal from the OLT is modulated at the ONU and sent back in upstream direction. At the OLT, a WDM receiver is used to demultiplex and detect the received optical signal from each ONU.

The optical output power of the OLT must be large enough to handle the upstream as well as the downstream transmission. The disparities in the received signals will be increased by the fact that the light must make a round-trip through the outside plant. The one-way loss in a power-splitting PON (PSPON) can vary by as much as $15 \mathrm{~dB}$ [ITU-T G.983.1, p. 14]. In an optical loopback network the total loss increases by a factor of 2 (complete path in forward and backward direction) to $30 \mathrm{~dB}$, if the losses are completely correlated. However, the correlation should not be complete because some of the loss variation results from differences in insertion losses, passive components, splice losses, and so on.

Therefore, we need means for calculating outside plant losses. The ITU recommendation [ITU-T G.982, p. 20] has provided a method for treating loss budgets statistically. In the approach, all components such as fiber, splices, splitters and connectors are assigned mean and standard deviation values. The total loss is within a range that can be calculated directly as follows

$$
\alpha_{\mathrm{t} \max }=\sum_{\mathrm{i}} \mathrm{n}_{\mathrm{i}}^{\max } \cdot \mathrm{L}_{\mathrm{i}}+\sqrt[3]{\sum_{\mathrm{i}} \mathrm{n}_{\mathrm{i}}^{\max } \cdot \sigma_{\mathrm{i}}^{2}}
$$

and 


$$
\alpha_{\text {tmin }}=\sum_{\mathrm{i}} \mathrm{n}_{\mathrm{i}}^{\min } \cdot \mathrm{L}_{\mathrm{i}}-\sqrt[3]{\sum_{\mathrm{i}} \mathrm{n}_{\mathrm{i}}^{\min } \cdot \sigma_{\mathrm{i}}^{2}}
$$

where $n_{i}$ is the number of components of type $i$ or for fiber it is the number of $k m . L_{i}$ is the mean loss of component $\mathrm{i}$ or for fiber the mean loss per $\mathrm{km}$ and $\sigma_{\mathrm{i}}$ is the standard deviation loss of component i. The standardization body ETSI has provided the same method for handling loss budgets statistically [ETSI97]. In the ETSI approach, the numbers of splices and connectors are also specified as the mean and standard deviation values for common passive optical components.

For example, a 1:32 splitter has a mean and standard deviation loss of $17 \mathrm{~dB}$ and $0.9 \mathrm{~dB}$ respectively. The round-trip fiber path can be up to $40 \mathrm{~km}$ long (see also section 4.4 ), and we assume the minimum to be $0.2 \mathrm{~km}$.

In the ETSI approach, L and $\sigma$ for the AWGM is not specified. After reviewing some specifications for commercially available products, we find $\mathrm{L}=2.75 \mathrm{~dB}$ and $\sigma=0.67 \mathrm{~dB}$. We find a round-trip loss of an optical loopback system with the parameters stated before of

$$
9.5 \mathrm{~dB} \leq \alpha_{\text {round-trip }} \leq 32.2 \mathrm{~dB}
$$

which leads to a maximum differential path loss of $22.7 \mathrm{~dB}$. Approximately $3 \mathrm{~dB}$ loss variation must be added due to output power variation of the OLT source and any loss variations. This leads to an extremely total differential path loss of about $26 \mathrm{~dB}$.

Furthermore, a transmitted frame is divided between an upstream and a downstream portion. An equal division of the frame, for example, doubles the line rate in both directions. The higher line rate leads to lower receiver sensitivities, further hurting the power budget [Agrawal02].

Optical loopbacks uses the same optical wavelength for both upstream and downstream direction. Optical loopbacks must be separated on two different fibers to avoid unacceptable interference. Doubling the fiber count leads to increased costs of the fiber plant, maintenance and complicates installation. The number of ports on the AWGM must also be doubled $(2 \times 2 \mathrm{~N})$.

An important advantage of the optical loopback is the availability of the symmetrical bitrate.

\section{Spectral Slicing}

Another upstream choice is spectral slicing [Zirngibl95, Feldman97, Feldman98, Jung01, Oksanen02, Dutta04, p. 221 et seq.]. The architecture, shown in Figure 2.23, is also called LARNet (Local Access Router Network) architecture in the literature. At the ONU, a broad-spectrum source such as an inexpensive edge-emitting light emitting diode (LED) is used. The edge-emitting LED emits a broad spectrum of wavelength, centered around a single wavelength. When the LED is connected into one input port of the AWGM, the emitted light will match the optical passbands of the AWGM and the spectrum is sliced by the AWGM in the upstream direction. Depending on the input port, the wavelength which is sliced is different. At the output port of the AWGM, we observe many wavelength components, each corresponding to a broad input source from 
an ONU. At the OLT, a broadband receiver which can detect any spectral component of the broad-spectrum source is used. The OLT is thus able to receive from each ONU, but there is only one receiver. Therefore, the upstream channel must be shared by the ONUs using a scheme like TDMA or dynamic bandwidth allocation [Kramer02, Kramer05].

Spectral slicing is attractive because edge-emitting LEDs are simple sources and lowpriced compared with tunable lasers. Commercially available LEDs, which can couple about $-10 \mathrm{dBm}$ into a single mode fiber are available. Unfortunately, spectral slicing of a broad-spectrum source of an AWGM leads to very high power loss, which leads to a reduced OLT to ONU distance.

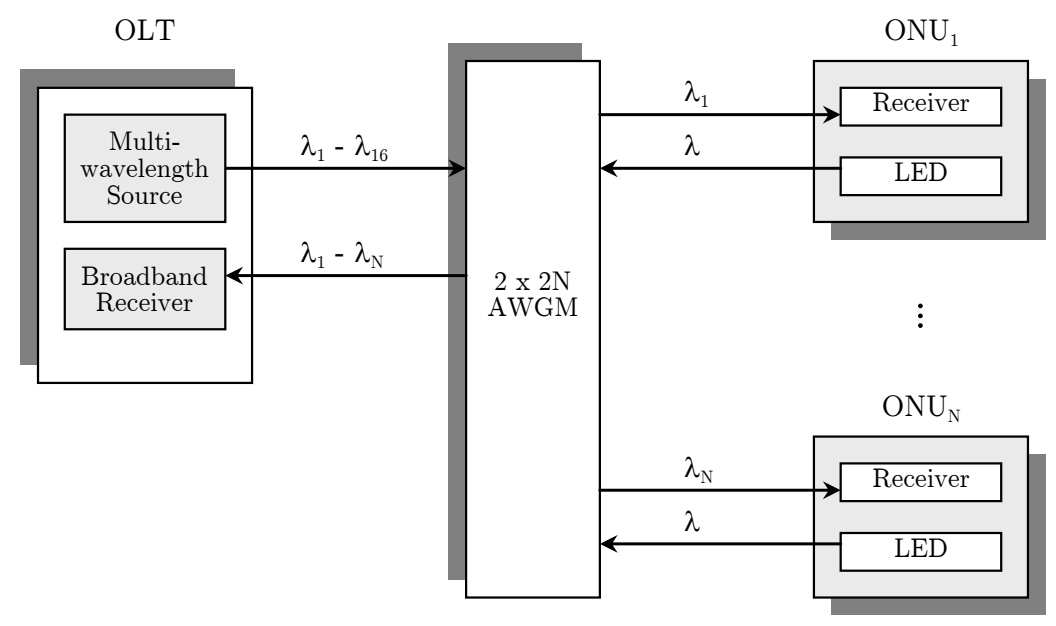

Figure 2.23: Schematic of a WDM PON with spectral slicing. Each ONU sends with a broad-spectrum source, whose spectrum is sliced by the AWGM in the upstream direction. At the OLT, a broadband receiver is required.

The primary objective of this work is to analyze, simulate and introduce new network architectures. In the last two subsections, different technical possibilities for the optical upstream and downstream channels are discussed. Each of the upstream and downstream channel transmission possibility can be used in the introduced architectures in chapter 3,4 and 5 .

Generally, a fundamental subject in telecommunication networks is international standards. The following subsection provides a short overview of the influencing standards, related to a WDM PON. 


\subsubsection{Standardization}

An important requirement for a successful breakthrough is a significant investment of the research community in combined efforts to achieve standardized solutions [Vetter04]. Unlike the well standardized TDM PONs (EPON, BPON, GPON) of today, there are no standards for single- and multistage WDM PONs; this situation is the key issue of this work (chapter $3,4,5,6)$.

Implemented WDM PON testbeds often use similar standards. These WDM PON testbeds use the well standardized spectral grids for dense WDM (DWDM) applications (see appendix 11) standardized in the ITU-T G.694.1 series. Therein the nominal central frequency is independent of the channel spacings standardized at $193.1 \mathrm{THz}$ $(1552.52 \mathrm{~nm})$. The size of the wavelength domain is not restricted by the ITU-T G.694.1 standard but is in practice limited by the components used (e.g., traditional fiber with its $\mathrm{OH}$ water peak at the $1400 \mathrm{~nm}$ wavelength region). Typical channel spacings in DWDM systems are $200 \mathrm{GHz}, 100 \mathrm{GHz}$, and $50 \mathrm{GHz}$ corresponding to $1.6 \mathrm{~nm}, 0.8 \mathrm{~nm}$ and $0.4 \mathrm{~nm}$ respectively, with respect to the $1500 \mathrm{~nm}$ transmission window (Figure 2.24). Due to the small channel spacings, thousands of different optical channels can be used but lasers and passive filters are very costly and temperature sensitive [Kartalopoulos00, Tanis02, Dutta04, p. 191 et seq.].

Thus, in access networks there is another standard which allows cost-effective applications through a combination of uncooled lasers, relaxed laser wavelength selection tolerances and wide pass-band filters. This standard is known as coarse WDM (CWDM) and is standardized in ITU-T G.694.2 (see appendix 11). In the case of CWDM systems, channel spacings of $20 \mathrm{~nm}$ are standardized. As a consequence, only 18 different wavelengths can simultaneously be used in one CWDM based network in the wavelength domain between $1270 \mathrm{~nm}$ and $1610 \mathrm{~nm}$ (Figure 2.24). State-of-the-art research demonstrates extremely compact DFB laser modules for DWDM and CWDM in a wide temperature range [Atsushi04].

In Wide-WDM (WWDM) networks, the wavelength spacing is over $250 \mathrm{~nm}$ in the $1300 \mathrm{~nm}$ and $1500 \mathrm{~nm}$ regions. This is the most cost effective solution of all WDM schemes. By virtue of the maximum number of two optical channels (one for upstream and one for downstream), WWDM is not a topic in this work. 


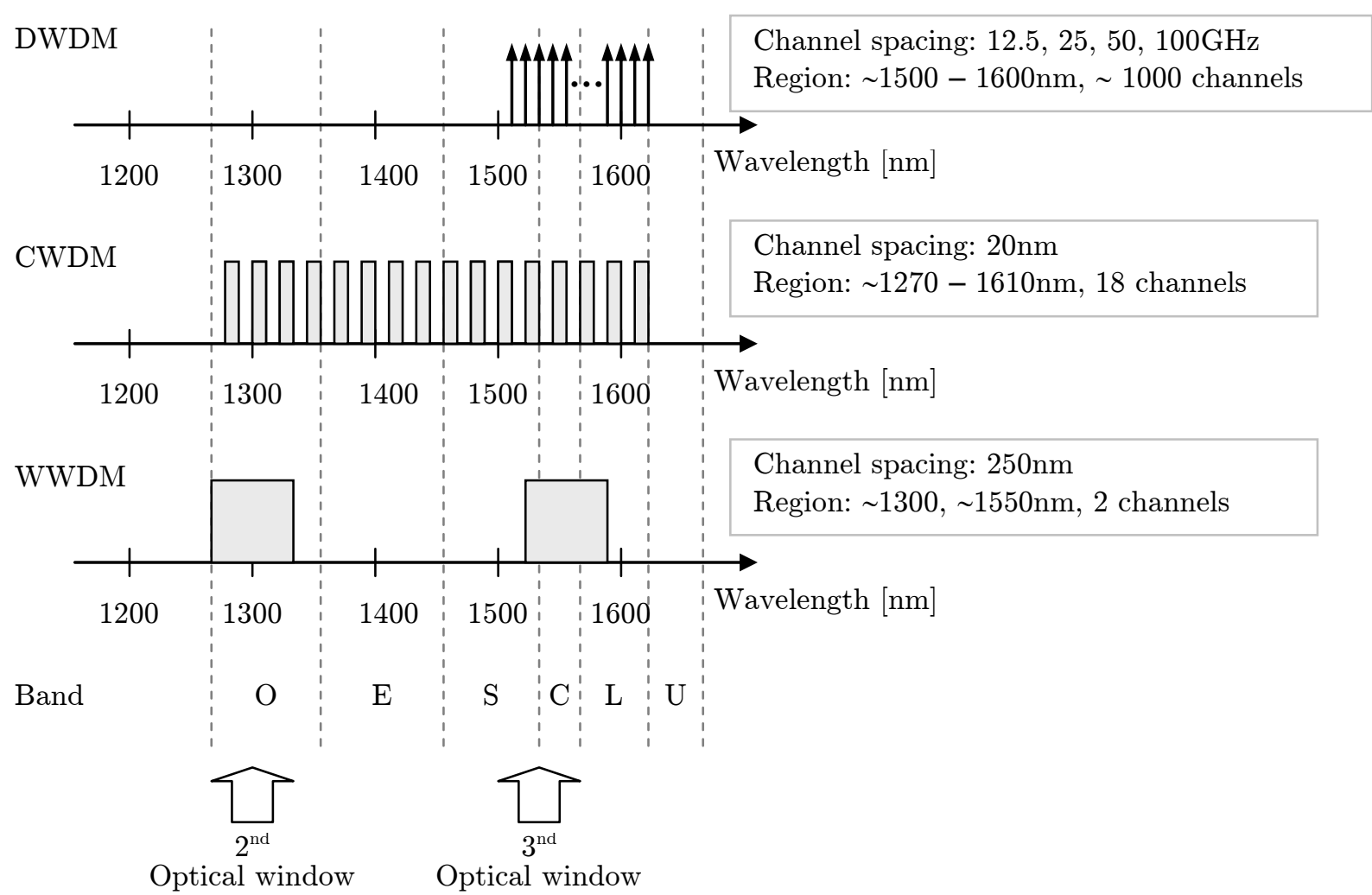

Figure 2.24: Comparison of different standardized WDM schemes and its correspondent wavelength grids. In their G.6xx recommendations the ITU defined these wavelength partitions. Wavelengths regions are denoted by O (Original), E (Extended), S (Short Wavelength), C (Conventional), L (Long), U (Ultralong).

Own standards for management and control interface specifications do not exist for WDM PONs. Preferred protocols like Gigabit Ethernet, ATM and so on, are not standardized. In many cases, protection features could be an issue [Wang04]. Basic approaches which could be adopted and implemented in WDM PONs, can be found in ITU-T G.983.6.

In this work we are using a generalized model to describe required wavelengths (see section 3.1). Therefore, it is not necessary to determine wavelengths which correspond with the ITU wavelength grid. However, later on in subsection 4.1.1 we will introduce a parameter "number of wavelengths in a fiber" $\left(\mathrm{n}_{\omega}\right)$. This parameter determines the needed channel spacing and therefore the potential ITU wavelength grid. 


\subsection{Paradigm Shift}

The paradigm shift, introduced in this chapter should separate this work into previous and future mindsets. From that time, the paradigm shift is used continuously in this work. At the end of this section, the characteristics with its assets and drawbacks will be discussed.

\subsubsection{New Paradigm}

In the past, for each service, a specialized telecommunication network was built. An example is the voice network, which was originally designed to transport only voice services. Each telecommunication network was optimized for a respective service. Thus, the utilization of the network with other services was undesirable. For the first time, with ISDN (Integrated Services Digital Network) [Kanbach99], the paradigm "one service - one network" is discarded.

The objective of the new paradigm is to transport all services in the past and future over a single telecommunication network. Today, we know that ISDN, xDSL and other up to date wired network technologies are due to the already enormous and still rocketing bandwidth demand overtaken by the internet.

In the near future, broadband access networks will be required with data rates over $1 \mathrm{Gbit} / \mathrm{s}$ for each customer. Due to this aspect the widespread deployment of fiber-tothe-home (FTTH) technology is speeding up [Lin06]. Currently, time-division multiple access passive optical networks (TDMA-PONs) are deployed. However, TDMA-PONs, like ATM PON (APON), Broadband PON (BPON), Generalized PON (GPON) and Ethernet PON (EPON) cannot keep up with the requirements for the broadcasting of lots of HDTV channels and the unicasting of several triple-play services (voice, data and video) [Söderlund04].

In contrast, wavelength-division-multiplexed PONs (WDM PONs) will be able to provide these required high data rates for each user with, however, the limitation of higher costs compared with TDM-PONs.

In contrast with cutting-edge scientific papers [Maier00, Bock04, Banerjee05, An04] is the focus to provide each user with at least one wavelength. This paradigm can be formulated as follows

$$
\# \text { user } \leq \# \text { service } \leq \# \lambda
$$

so that each user and each service obtain at least one wavelength (see Figure 2.25). A user receives the status of a user when he receives at least one service and, therefore, he receives at least one wavelength. In the case of telecommunications, the term "service" is a notation to characterize a telecommunication network that is able to carry information. Information is able to eliminate indefiniteness or rather it is able to 
eliminate uncertainty with disclosure, communication or with knowledge over objects and phenomena.

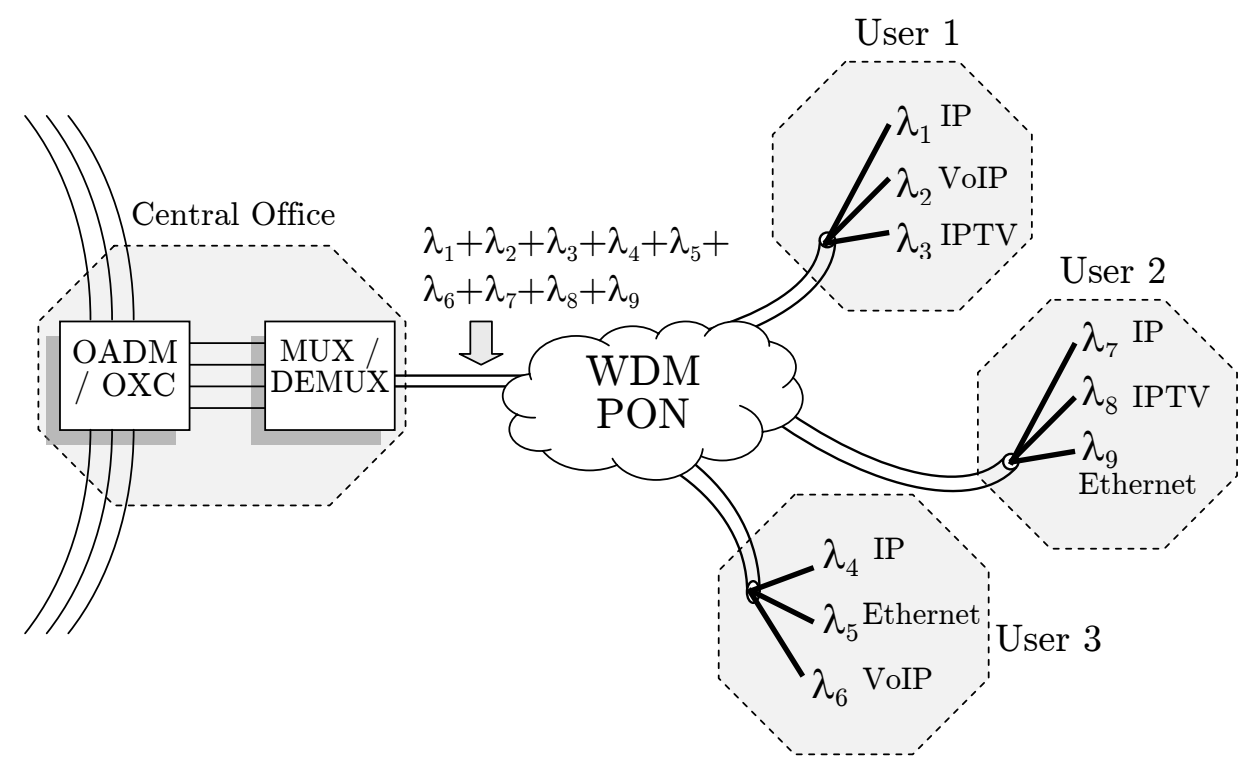

Figure 2.25: The paradigm shift: at least one wavelength for each user. Each wavelength transports at least one service.

\subsubsection{Characteristics}

This work focuses on access networks. In this case, another motivation is present to deploy the paradigm in (3.1):

and

$$
\text { independent multi provider solution }
$$

independent multi service solution.

Multiple providers must be able to transport their services over the same network to their customers. Due to the ability to transport more than one wavelength and therefore more than one service to each user, the unbundling of services is possible and regularly practicable. As we already recognized, the possibility of unbundling is an important property. Due to the unbundling it is possible to distinguish between a network operator and many internet service providers (ISP). A separation between the network operator and ISPs leads to sharing the cost of the physical network over all ISPs.

In many research labs, WDM PON access networks are considered as the nextgeneration optical access. There are several obvious reasons for this:

(1) Higher bandwidth: each dedicated wavelength for each ONU can have its own maximum downstream and upstream bitrate (no sharing as in TDM PONs). Each ONU can have more than one wavelength which leads to a higher and scalable bitrate for each individual ONU. 
(2) No sharing:

(3) Flexible bitrate:

(5) Security:

(6) Collisions:

(7) Simplicity:

(8) Performance:

(9) Fairness (ONU):

(10) Fairness (service):

(11) Upgrade (channel):
(4) Transparency:

each dedicated wavelength connects the OLT and the ONU and transports one (or more) service(s) without sharing with other ONUs.

bitrate on each dedicated wavelength channel can be different.

the format on each wavelength channel can be different.

no signal sharing with other ONUs leads to a higher security.

in the upstream direction there is no need for ranging or collision avoidance.

no ranging and collision avoidance leads to a simpler OLT and ONU design.

no ranging and collision avoidance leads to a higher performance.

no channel sharing with other ONUs leads to fairness between ONUs.

thanks to the paradigm shift in (3.1), it is possible to transport each service on a different optical channel. Each optical channel is independent from each other which leads to fairness between different services.

each dedicated optical channel can be upgraded in terms of a higher bitrate.

(12) Upgrade (wavelength): thanks to the paradigm shift, the number of wavelengths for each ONU can be upgraded.

(13) Separate: operator and an internet service provider (ISP).

However, there still are some drawbacks of WDM PON networks. WDM components such as multiplexers/demultiplexers, wavelength routers, multiwavelength sources are more expensive compared with TDM PON components. Yet the cost for WDM components is rapidly decreasing.

Due to the cost sensitivity in access networks it is important to introduce new architectures that are able to share components and costs. Therefore, it is important to identify WDM PON architectures, which minimize costs. These architectures are identified in a techno-economic evaluation in chapter 5, based on a developed design tool. The design tool automatically generates only architectures that are technologically feasible and therefore have a practical application. 
A topic in a WDM PON is the assignment of wavelengths to ONUs or to services [McGarry06]. This layer two task is part of the medium access control (MAC) protocol and does not affect our current work scope. We focus on practical WDM PON architectures which allow the transportation of many different wavelengths collision free from the OLT to each ONU.

The next section gives an overview of state-of-the-art optical access networks which leads to the requirements of future optical access networks.

\subsection{Summary}

The motivation of this work is to initiate the paradigm shift introduced in section 2.5. This means each user and each service obtains at least one wavelength. From another point of view, this work represents a generalization of the special case: one wavelength per user for all services.

The landscape of telecommunications, especially in the field of access networks, is now undergoing a major change towards FTTH. This major change is accepted worldwide and is driven from cutting-edge countries like Sweden, Korea, California, Japan, and projects like the Utah Telecommunications Open Infrastructure Agency (UTOPIA) in the USA or the Multi-Service Access Everywhere (MUSE) in the Netherlands and many others [Maeda04, Lin06].

TDM-PONs have limited bitrates per user. They are complicated to upgrade, and all ONUs must work at the aggregate bit-rate. Privacy is an important issue due to the broadcast of the downstream information. Network integrity is a field of study because one ONU can corrupt the entire upstream transmission.

A delicate operational problem is how TDM PONs will be upgraded in the future in terms of higher bitrates per user. Novel fiber infrastructure technologies like Blown Fibre enable significant cost savings in the central office (40\%), distribution network and in the customer drop [Faulkner04].

The growing demands for more and higher quality services lead to WDM PONs which are capable of providing higher bit rates than TDM PONs are able to [Bradley99, Salvador99, Kyeong02].

In Korea, KT (former Korea Telecom) has demonstrated technological capability and economical feasibility to commercialize WDM-PONs [Lin06, p. 104]. As part of their strategy, KT will foster WDM-PONs in Kwang-ju on a large scale for the first time in the world. As far as we can see from the examples, there will be a worldwide need for WDM PONs in the future [Sivalingam05, p. 61 et seq.].

WDM can be exploited in access networks to enhance capacity. However, the costs of WDM components are shared among fewer users and leads to higher costs. A challenge would be to investigate how to migrate from a TDM-PON to a WDM-PON in a costeffective, scalable and flexible way. What architecture is the best related to costs, flexibility, upgradeability and is future proof? What are the parameters that influence 
the deployment of such a technology? In chapter 5, a techno-economic analysis allows a better realization of the influencing parameters.

A separate issue is how to provide open access for multiple ISPs to share the same fiber infrastructure when the local legislation requires it. With WDM PON networks and the introduced paradigm shift in the next chapter it is possible to allow a multi ISP solution over the same fiber infrastructure.

An overview of different TDM PON technologies compared to WDM PON is given in Table 2.2. In the next chapter, the paradigm shift and its pros and cons are discussed.

\begin{tabular}{|c|c|c|c|c|}
\hline & $\mathrm{A} / \mathrm{BPON}$ & GPON & EPON (EFM) & WDM PON \\
\hline Standard & ITU G.983 & ITU G.984 & IEEE 802.3ah & None \\
\hline Approved & $1998 / 2001$ & 2003 & 2004 & None \\
\hline Max. datarate & $622 / 155 \mathrm{Mbit} / \mathrm{s}$ & 1.24 or $2.488 \mathrm{Gbit} / \mathrm{s}$ & $1.25 \mathrm{Gbit} / \mathrm{s}$ & $>1 \mathrm{Tbit} / \mathrm{s}$ \\
\hline Packet/cell size & 53 Bytes & $53-1518$ Bytes & $64-1518$ Bytes & Protocol dependent \\
\hline Max. PON splits & $\leq 32$ & $\leq 64$ & $\leq 16$ & 100 's \\
\hline Reach & $10 / 20 \mathrm{~km}$ & $20 \mathrm{~km}$ & $10 \mathrm{~km}$ & also $>20 \mathrm{~km}$ \\
\hline Layer 2 Traffic & ATM & Generic & Ethernet & not specified \\
\hline Voice & TDM & TDM & VoIP or TDM & VoIP or TDM \\
\hline Video & RF overlay & $\mathrm{RF}$ or IP & RF overlay & RF or IP \\
\hline Strengths & $\begin{array}{l}\text { - } \mathrm{QoS} \\
\text { - Mature standards } \\
\text { - SDH integration }\end{array}$ & $\begin{array}{l}\text { - Efficiency } \\
\text { - Flexibility }\end{array}$ & $\begin{array}{l}\text { - Low cost chipsets } \\
\text { - Easy integration }\end{array}$ & $\begin{array}{l}\text { - Future proof } \\
\text { - Flexibility } \\
\text { - Extensibility }\end{array}$ \\
\hline Bandwidth efficiency & $\begin{array}{l}<75 \% \\
28 \mathrm{~dB}\end{array}$ & $\begin{array}{l}>90 \% \\
28 \mathrm{~dB}\end{array}$ & $\begin{array}{l}<75 \% \\
24 \mathrm{~dB}\end{array}$ & Protocol dependent \\
\hline $\begin{array}{l}\text { Network } \\
\text { synchronisation }\end{array}$ & Specified & Specified & Left to vendor & not specified \\
\hline Security & Specified & Specified & Left to vendor & not specified \\
\hline Attendees & $\begin{array}{l}\text { - Service Provider } \\
\text { focused }\end{array}$ & $\begin{array}{l}\text { - Service Provider } \\
\text { focused }\end{array}$ & $\begin{array}{l}\text { - Enterprise and } \\
\text { consumer market } \\
\text { focused }\end{array}$ & $\begin{array}{l}\text { - Service Provider } \\
\text { - Enterprise } \\
\text { - consumer market } \\
\text { focused }\end{array}$ \\
\hline Estimated cost & Low & Medium & Lowest & High \\
\hline
\end{tabular}

Table 2.2: Overview of different TDM PON technologies compared to WDM PON. 


\section{Single-Stage Networks}

$\mathrm{T}$

he goal of this chapter is to define rules to calculate single-stage WDM PON based access networks. These rules can be used as a basis for a multi-stage WDM PON, for techno-economic calculations and to dimension such future WDM PON based access networks. The behavior of optical networks is analog in nature [Hecht02, Milorad04, Born05]; however, this work considers the behavior as digital to analyze functions.

The main difference compared with state-of-the-art academic works (e.g. [Maier00, Bock04, Banerjee05]) is the focus on providing each ONU with more than one wavelength (see section 2.5). When the network provides the transportation of more than one wavelength per ONU, a lot of advantages can be identified (see subsection 2.5.2). It enables a pay-as-you-grow strategy by starting with one wavelength per ONU. In dependence of the requirements, wavelengths can be added for individual ONUs. Most significantly this work represents a generalization of the special case: one wavelength per ONU. Another essential difference is the usage of theoretically any number of stages compared with other publications such as [Parker98] (see chapter 4).

\subsection{Wavelength reuse and space demultiplexing}

In order to be in a position to study and define the connectivity properties of the WR, it is useful to consider optical channels routed by a WR as belonging to a discrete bidimensional domain: space and wavelength. It must be noted that the WR is a passive device and is able to perform space permutations while it does not modify the wavelength of the signals. The input and output ports of the WR are numbered by a progressive integer index.

The overall transfer function of the WR is represented in (4.13). The WR behaves like a periodical pass-band filter. Its transfer function peaks repeats at fixed wavelength intervals called free-spectral-range (FSR). Designing the WR we make use of the following equation [Glance94, Smit96]

$$
\mathrm{FSR}=\mathrm{M} \cdot \Delta \lambda \quad \mathrm{M} \in \mathbb{N} .
$$

In the real world, the FSR has a more complicated expression. The FSR and the transfer function are not constant and vary over the $\lambda$-axis. The following discussion is based on an idealized WR model. The wavelength domain for the channels routed by the WR is a discrete set of wavelengths, numbered by an index $\mathrm{k}$ that can assume all 
the integer values from 1 to (theoretically) infinity (Figure 3.1). Within optical networks, the bandwidth is divided into frequency bands numbered by positive integers $\mathrm{k}$. Wavelengths are spaced by a wavelength interval $\Delta \lambda$, whereas $\lambda_{0}$ correlates with $\mathrm{k}=1$ and represents the lowest useable wavelength. Every wavelength used depends on the wavelength number $\mathrm{k}$ and the wavelength interval $\Delta \lambda$ and can be expressed by

$$
\lambda(\mathrm{k})=\lambda_{0}+(\mathrm{k}-1) \Delta \lambda \quad \forall \mathrm{k} \in[1, \infty]
$$

The base wavelength $\lambda_{0}$ is the lowest wavelength that appears at port 1 for all WRs. Thus, the wavelength domain defined for one single WR can be extended to the entire WDM PON. That will lead to the same routing characteristic for all WR.

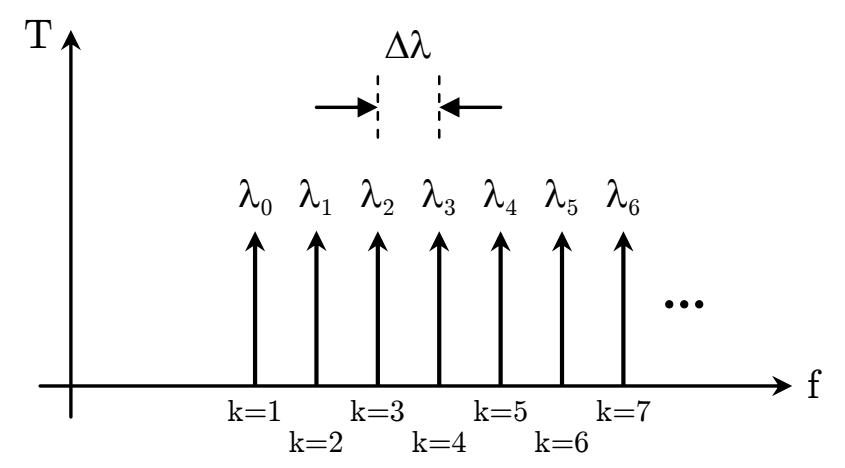

Figure 3.1: Discrete wavelength domain in a WDM PON.

In Figure 3.2, an example of the transfer function of a WR is depicted. It illustrates the transfer function from all the inputs to a certain output port. The wavelengths that match the transfer function from an input $\mathrm{i}$ to an output o with the same index $\mathrm{i}$ is called the base wavelength $\lambda_{0}$ (Figure 3.2). The wavelength channels that are routed by the WR in the wavelength domain can now be defined as a discrete set of wavelengths. This set of wavelengths is numbered by the index $\mathrm{k}$ from 1 (base wavelength) to theoretically infinity.

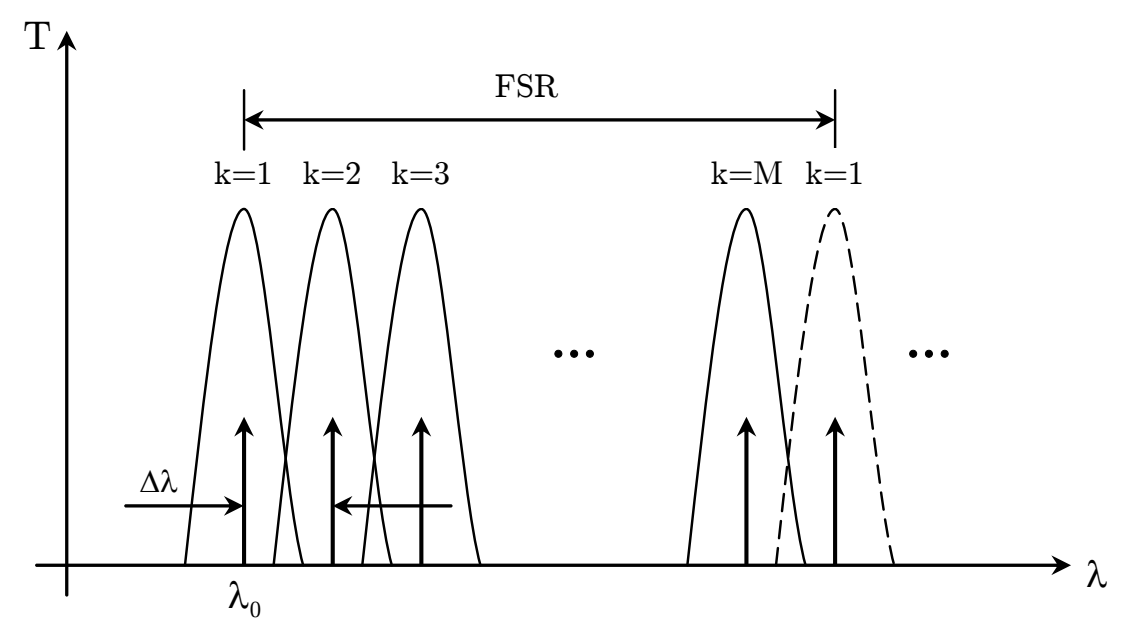

Figure 3.2: Transfer function from all the inputs to a certain output port of a WR with coarseness $\mathrm{c}=1$ and base wavelength $\lambda_{0}$. For simplicity, only one FSR is shown. 
In this example, only one discrete wavelength is routed to the same output port. WR can also be constructed so that more than one wavelength can be routed to the same output port (Figure 3.3). We introduce a second parameter to characterize a WR which is called coarseness $\mathrm{c}$ and represents the number of adjacent wavelength channels belonging to the wavelength comb routable on the same output port.

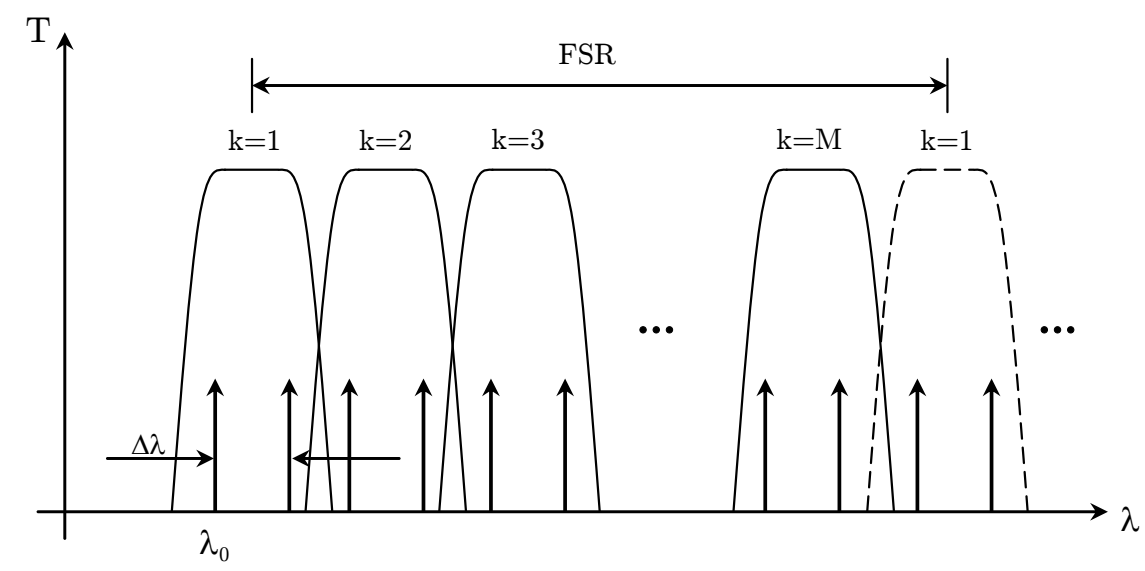

Figure 3.3: Transfer function from all the inputs to output port 1 of a WR with coarseness $\mathrm{c}=2$ and base wavlength $\lambda_{0}$.

In summary, a WR can be specified by the size $M$ and the coarseness c. The WR routing function is given by [Barry93]:

$$
\mathrm{o}=1+\left(\mathrm{i}-1+\left\lfloor\frac{\mathrm{k}-1}{\mathrm{c}}\right\rfloor\right) \bmod \mathrm{M}
$$

where $o, i \in[1, M]$ represents the output and input port and $k \in[1, \infty]$ represents the discrete wavelength number (Figure 3.1). In equation (4.3) the important modulo-M division is a consequence of the periodical behavior of the WR and is named "cyclicrouting". In the frequency domain, the cycling-routing property appears as the FSR.

In conclusion, a WR can be characterized by the size $\mathrm{M}$ and the coarseness c. Figure 3.4 (a) shows us an example of a WR with $\mathrm{M}=4, \mathrm{c}=1$ and with three wavelengths on input port one and four. The wavelengths $\lambda_{1}{ }^{1}, \lambda_{2}{ }^{1}$ and $\lambda_{3}{ }^{1}$ on the input port 1 are routed to the output ports 1, 2 and 3 respectively. The same wavelengths $\lambda_{1}^{4}, \lambda_{2}^{4}$ and $\lambda_{3}^{4}$ on the input port 4 are routed to the output ports 4,1 and 2 . As we can see, the wavelengths can be reused without collision as long as they enter from different input ports. This is another important property of a WR. In Figure 3.4 (b) an example of a WR with $\mathrm{M}=4, \mathrm{c}=2$ and with different numbers of wavelengths are reported. Due to the coarseness factor of $c=2$, two consecutive wavelengths of a certain input port are routed to the same output port. In Figure 3.4, we can see the important cyclic-routing property $\left(\lambda_{2}{ }^{4}, \lambda_{3}{ }^{4}\right.$ in Figure 3.4 (a) and $\lambda_{3}{ }^{4}, \lambda_{4}{ }^{4}$ in Figure $\left.3.4(\mathrm{~b})\right)$. 


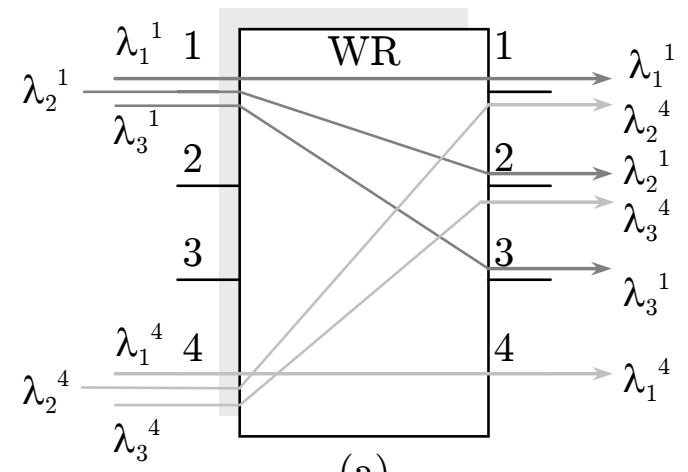

(a)

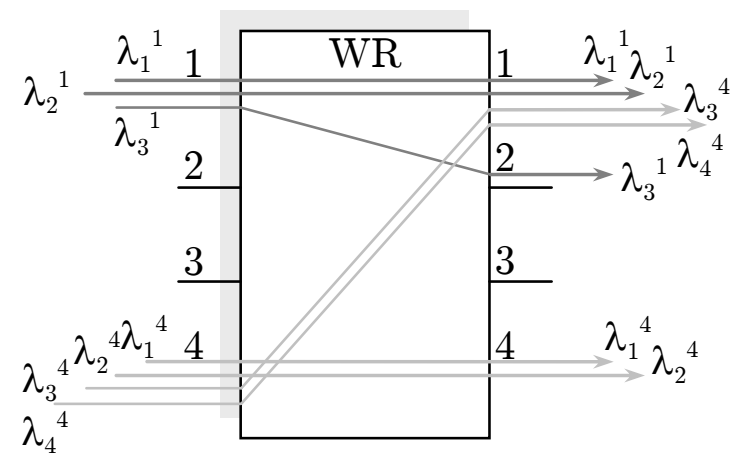

(b)

Figure 3.4: WR routing example with $\mathrm{M}=4$ and coarseness: (a) $\mathrm{c}=1$ and (b) $\mathrm{c}=2$.

In Figure 3.4, the same wavelengths on different input ports can be used simultaneously due to the wavelength reuse property of the WR. This important property is independent from the chosen input pattern.

On the basis of the routing properties of the WR, we are now going to define rules to evaluate the WR parameters in a WDM PON.

In this work, we will consider only WDM PONs in which only some of its inputs but all of the outputs of the WR are used. This means that M must be always greater than I and equal with the number of output ports $\mathrm{O}$. We examine only WR in which the number of inputs is equal to the number of outputs. This implies that the space demultiplexing function in single-stage WDM PONs is performed if the following condition holds:

$$
\mathrm{I}<\mathrm{O} \leq \mathrm{M}
$$

The space demultiplexing function depends on the choice of the input occupancy pattern. Thus, we must define different input patterns for a WR. In the past, only designated input occupancy patterns have been used [Maier00]. Thus, we define a generic input pattern for a WR. The generically terminated input port $\tilde{i}$ in an input pattern can be described as follows:

$$
\tilde{\mathrm{i}}=\left\lfloor\frac{2 \cdot \mathrm{I} \cdot \bmod \left(2^{\mathrm{O}}\right)}{2^{\mathrm{O}}}\right\rfloor \cdot \mathrm{o} \quad \mathrm{I} \in\left[0,2^{\mathrm{M}}-1\right], \quad \mathrm{o} \in[1, \mathrm{M}], \quad \tilde{\mathrm{i}} \in[0, \mathrm{M}]
$$


where I is the terminated input pattern described as a decimal number. The expression in the round-off brackets results in two numbers: one and zero. The result is multiplied by the output port number o so that $\tilde{\mathrm{i}} \in[0, \mathrm{M}]$. The identifier $\tilde{\mathrm{i}}$ depends on the two parameters input occupancy pattern and the number of the used output ports. Substitution (4.5) in (4.3) leads to

$$
\mathrm{o}=1+\left(\left\lfloor\frac{2 \cdot \mathrm{I} \cdot \bmod \left(2^{\mathrm{o}}\right)}{2^{\mathrm{o}}}\right\rfloor \cdot \mathrm{o}-1+\left\lfloor\frac{\mathrm{k}-1}{\mathrm{c}}\right\rfloor\right) \bmod \mathrm{M} .
$$

The commonly known inverse function of $a=b \bmod c$ is $b=c \cdot q+a$ where $q \in[0, \infty]$. By utilizing this correlation in equation (4.6), we obtain

$$
\mathrm{Mq}+\mathrm{o}-1=\left\lfloor\frac{2 \cdot \mathrm{I} \cdot \bmod \left(2^{\mathrm{o}}\right)}{2^{\mathrm{o}}}\right\rfloor \cdot \mathrm{o}-1+\left\lfloor\frac{\mathrm{k}-1}{\mathrm{c}}\right\rfloor+\mathrm{z}
$$

Each channel inside a consecutive wavelength comb is indexed by the number $\mathrm{z}$ in (4.7). Multiplying equation (4.7) with $\mathrm{c}$ and transformation to $\mathrm{k}$ leads to

$$
\mathrm{k}=\mathrm{Mqc}+(\mathrm{o}-1) \mathrm{c}-\left(\left\lfloor\frac{2 \cdot \mathrm{I} \cdot \bmod \left(2^{\mathrm{o}}\right)}{2^{\mathrm{o}}}\right\rfloor \cdot \mathrm{o}-1\right) \mathrm{c}+1+\mathrm{z} .
$$

The result of equation (4.5) leads to figures between zero and M. The parameter i in equation (4.3) is defined between one and M. Thus, equation (4.8) must be adjusted for the case $\tilde{\mathrm{i}}=0$. This can be done by multiplying (4.8) with an expression so that the result of (4.8) is zero for the case $\tilde{i}=0$. The expression in the round-off brackets in (4.5) fulfills our requirements, and $\mathrm{k}$ is then determined by

$$
\mathrm{k}=\left(\mathrm{Mqc}+(\mathrm{o}-1) \mathrm{c}-\left(\left\lfloor\frac{2 \cdot \mathrm{I} \cdot \bmod \left(2^{\mathrm{o}}\right)}{2^{\mathrm{o}}}\right\rfloor \cdot \mathrm{o}-1\right) \mathrm{c}+1+\mathrm{z}\right) \cdot\left\lfloor\frac{2 \cdot \mathrm{I} \cdot \bmod \left(2^{\mathrm{o}}\right)}{2^{\mathrm{o}}}\right\rfloor .
$$

With the equation (4.9) we can evaluate the set of all wavelengths that, being inserted into the WR from any of its used input port occupancy patterns, reach a given output port o. It depends only on the coarseness $\mathrm{c}$, the size $\mathrm{M}$ and the input occupancy pattern I of the WR. In the next section a possible method to describe the behavior of a WR based on wavelength transfer matrices is described. 


\subsection{Wavelength Transfer Matrix}

Single-stage WDM PONs use one single WR and therefore consist of only one stage (Figure 3.5). Single-stage WDM PONs are simple to understand and are easier to calculate than multistage architectures. Single-stage networks require only single wavelength sources. Additionally, they have a more convenient power budget compared to multistage WDM PON networks.

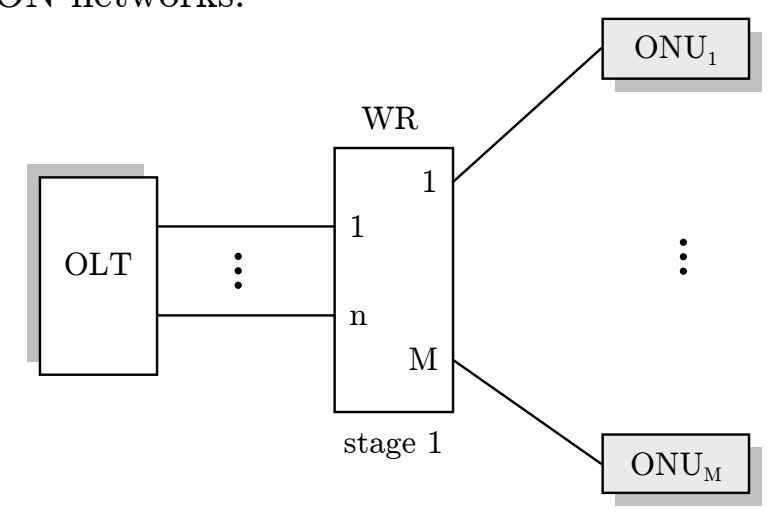

Figure 3.5: Architecture of a single-stage WDM PON.

The preferred embodiment of the wavelength router (WR) is the arrayed-waveguidegrating multiplexer (AWGM). As mentioned in subsection 2.4.1, the WR can be deployed as a multiplexer, demultiplexer and as a static wavelength router.

The WR can be logically seen as a combination of $\mathrm{n}$ demultiplexers und $\mathrm{n}$ multiplexers (see Figure 2.17 in subsection 2.4.1). However, it is essentially an analog grating based element with severe limitations in its size due to crosstalk properties [Monroy02]. Possible applications of it have been described by Y. Tachikawa [Tachikawa96]. One elementary property is that each wavelength on an individual input port of the WR appears only on one output port. Thus, all routing is done by the selection of the input port and the input wavelength.

The output matrix $\mathrm{O}_{\mathrm{M}}$ of the WR is a product of the Wavelength Transfer Matrix $\mathrm{WTM}_{\mathrm{M}, \mathrm{M}}$ and the input matrix $\mathrm{I}_{\mathrm{M}}$, whereby $\mathrm{M}$ is the size of the WR [Barry93, Oguchi96]

$$
\mathrm{O}_{\mathrm{M}}=\mathrm{WTM}_{\mathrm{M}, \mathrm{M}} \cdot \mathrm{I}_{\mathrm{M}} .
$$

The product of the elements of the input matrix $\mathrm{I}_{\mathrm{M}}$ and the WTM is defined as follows

$$
\begin{gathered}
\Lambda^{\mathrm{k}} \cdot \lambda_{\mathrm{i}}^{\mathrm{k}}=\lambda_{\mathrm{i}}^{\mathrm{k}} \\
\Lambda^{\mathrm{l}} \cdot \lambda_{\mathrm{i}}^{\mathrm{k}}=0 \quad(\mathrm{l} \neq \mathrm{k})
\end{gathered}
$$

where $\Lambda^{\mathrm{k}}$ are the elements of the WTM and $\lambda_{\mathrm{i}}^{\mathrm{k}}$ are the elements of the input matrix $\mathrm{I}_{\mathrm{M}}$. Only those elements with the same superscripted index result in elements in the output matrix with the same wavelength. For a WR with a dimension of $\mathrm{M}=4$, the WTM is given by 


$$
\mathrm{WTM}_{4,4}=\left[\begin{array}{cccc}
\Lambda^{1} & \Lambda^{4} & \Lambda^{3} & \Lambda^{2} \\
\Lambda^{2} & \Lambda^{1} & \Lambda^{4} & \Lambda^{3} \\
\Lambda^{3} & \Lambda^{2} & \Lambda^{1} & \Lambda^{4} \\
\Lambda^{4} & \Lambda^{3} & \Lambda^{2} & \Lambda^{1}
\end{array}\right]
$$

The WTM in (4.13) represents a matrix which is called a Latin squared matrix. Specifically, a Latin squared matrix consists of sets of $\mathrm{N}$ symbols arranged in such a way that no orthogonal (row or column) contains the same number twice. In the literature, routing devices with a Latin squared connection matrix are called a Latin router [Barry93]. The number of columns in (4.13) is equivalent to the used wavelengths per input port and the number of rows is like the size of the WR.

The example in (4.14) shows that input wavelengths are indicated in the range from $\lambda_{1}{ }^{1}$ to $\lambda_{4}{ }^{4}$, whereby the superscripted index is the input port number of the WR and the subscripted index is the wavelength number. This leads to the following input matrix $\mathrm{I}_{4,4}$ for a WR with $\mathrm{M}=4$ and 4 wavelengths per input port

$$
\mathrm{I}_{4,4}=\left[\begin{array}{cccc}
\lambda_{1}^{1} & \lambda_{1}^{2} & \lambda_{1}^{3} & \lambda_{1}^{4} \\
\lambda_{2}^{1} & \lambda_{2}^{2} & \lambda_{2}^{3} & \lambda_{2}^{4} \\
\lambda_{3}^{1} & 0 & \lambda_{3}^{3} & \lambda_{3}^{4} \\
\lambda_{4}^{1} & \lambda_{4}^{2} & \lambda_{4}^{3} & \lambda_{4}^{4}
\end{array}\right]
$$

The dimension of the input matrix in (4.14) varies in dependence on the size of the WR (columns) and the number of used wavelengths (rows). The total number of elements in the input matrix I is equal to the number of the entire wavelengths in the whole WDM PON. Wavelengths that are not used on a certain input port are set to zero as we can see in (4.14), whereas $\lambda_{3}{ }^{2}$ is set to zero, for example.

The output matrix can now be calculated by equation (4.10) and by keeping the conditions in (4.11) and (4.12). In this case, the dimension of the resulted matrix is $4 \mathrm{x}$ 4 and is given by

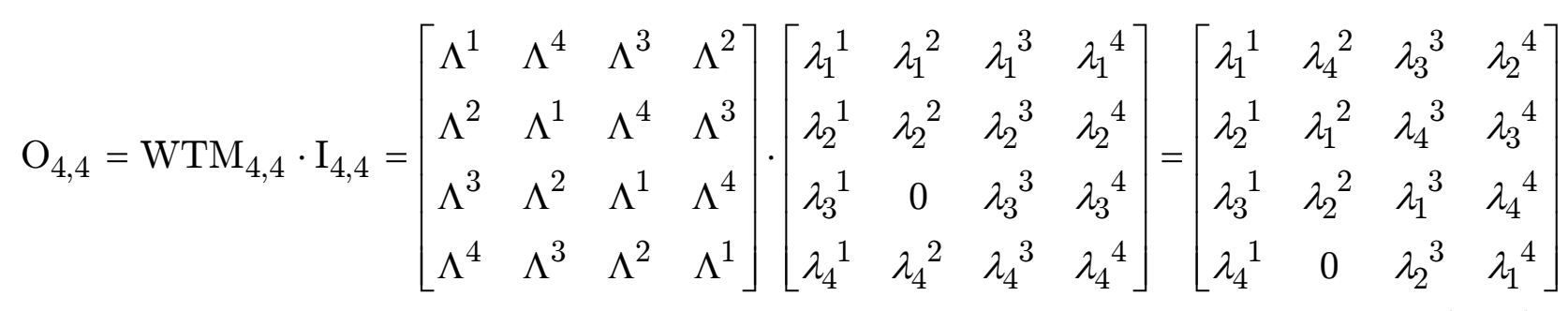

Note that the inputs $\lambda_{1}{ }^{1}$ to $\lambda_{4}{ }^{4}$ of the calculated output matrix are interlaced. We can see in (4.15) that all inputs at one input port appear interlaced at all output ports. For 
example, the inputs $\lambda_{1}{ }^{1}$ to $\lambda_{4}{ }^{1}$ at input port 1 (first column in (4.14)) appears at the output ports 1 to 4 (first column in the output matrix (4.15)). Wavelengths not used appear as zero elements in the output matrix. This is a nice property because the WTM is independent from the number of wavelengths and input ports used.

The equation (4.15) represents an example of a WDM PON network. This network is illustrated in Figure 3.6.

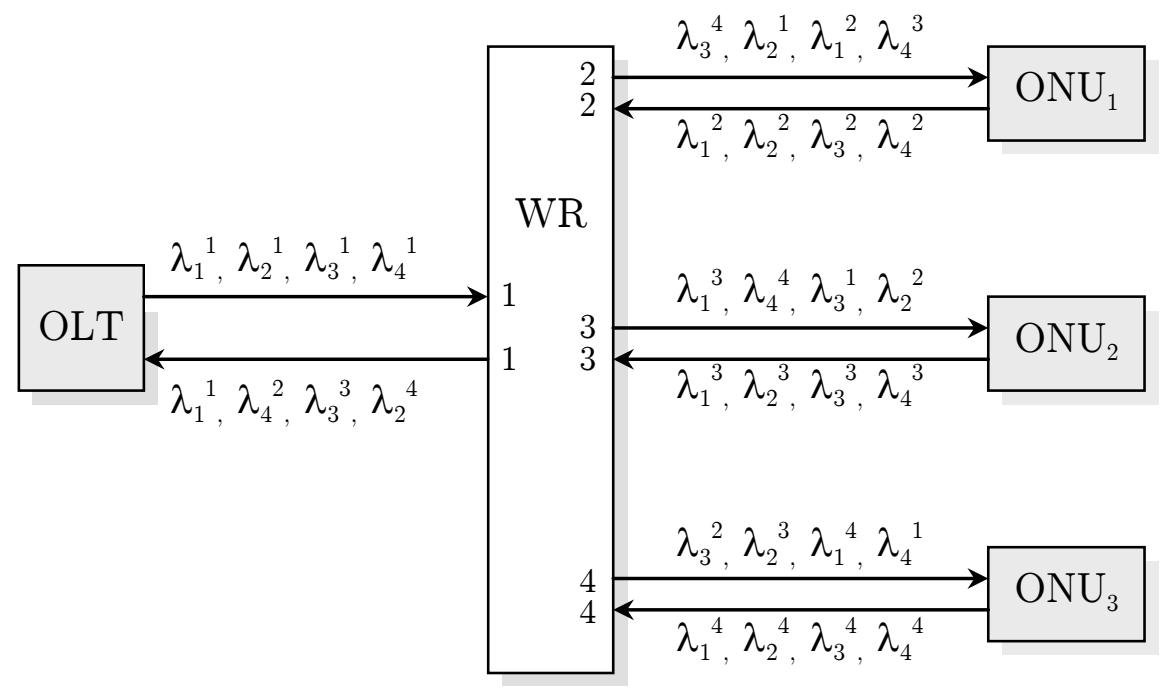

Figure 3.6: Example of a calculated single-stage WDM based PON with a size of the WR of $4(\mathrm{M})$, coarseness of 1 (c), number of wavelength per user of $4\left(\mathrm{U}_{\omega}\right)$, and number of users of $3(\mathrm{U})$.

All the input wavelengths $\lambda_{1}{ }^{1}$ to $\lambda_{4}{ }^{1}$ at the input port 1 (from the OLT) are routed to the output ports 1 to 4 . Therefore, the OLT can communicate with all other ONUs at a specific wavelength. On the other hand, each individual ONU can communicate with the OLT at a specific wavelength. Incidently, ONUs can also communicate with other ONUs at a specific wavelength if required.

Further potentialities can be achieved by optical networks which are equipped with wavelength conversion possibilities [Mukherjee97, p. 341 et seq., Mohammad03, p. 433 et seq.]. Due to the critical cost situation in optical access networks, wavelength conversion is not a topic in this work. Therefore, the discrete wavelength domain is identical over the whole WDM PON, because there is no possibility to lose or generate wavelengths within the network.

The architecture in Figure 3.6 show us a physical star topology. This topology can be considered as a set of virtual connections, represented by the different wavelengths $\lambda_{1}{ }^{1}$ to $\lambda_{4}{ }^{4}$. In Figure 3.7, the same four stations (OLT, $\left.\mathrm{ONU}_{1}-\mathrm{ONU}_{3}\right)$ as in Figure 3.6 are shown.

The different wavelengths form a bidirectional ring structure in Plane 0 and Plane 1 working in opposite direction. In Plane 2, there are rings which do not connect all stations. In this example, there are two rings on Plane 2. One ring connecting the OLT and $\mathrm{ONU}_{2}$, the other ring $\mathrm{ONU}_{1}$ and $\mathrm{ONU}_{3}$. It should be stated that four wavelengths $\left(\lambda_{1}{ }^{1}, \lambda_{3}{ }^{2}, \lambda_{1}{ }^{3}, \lambda_{3}{ }^{4}\right)$ are unutilized and do not contribute to forming logical rings. To avoid the creation of rings which do not connect all stations, [Woesner98] pointed out that the number of wavelengths has to be a prime number. With the number of wavelengths 
being a prime number, the network consists of "number of wavelengths"-1 rings in which all stations are connected to it [Woesner98].

As we can see in Figure 3.7, the number of wavelengths is not a prime number. As a consequence, the number of rings is smaller than the "number of wavelengths"-1.

Another interesting property can be achieved by varying the coarseness factor (see section 3.1) of the WR. If we imagine the coarseness factor $\mathrm{c}$ of the WR in Figure 3.7 is greater than one, the number of possible wavelengths that connect the stations are also greater than one. For example, with a coarseness factor of two, the number of possible rings is doubled. These rings can be used to form bidirectional redundant optical networks.
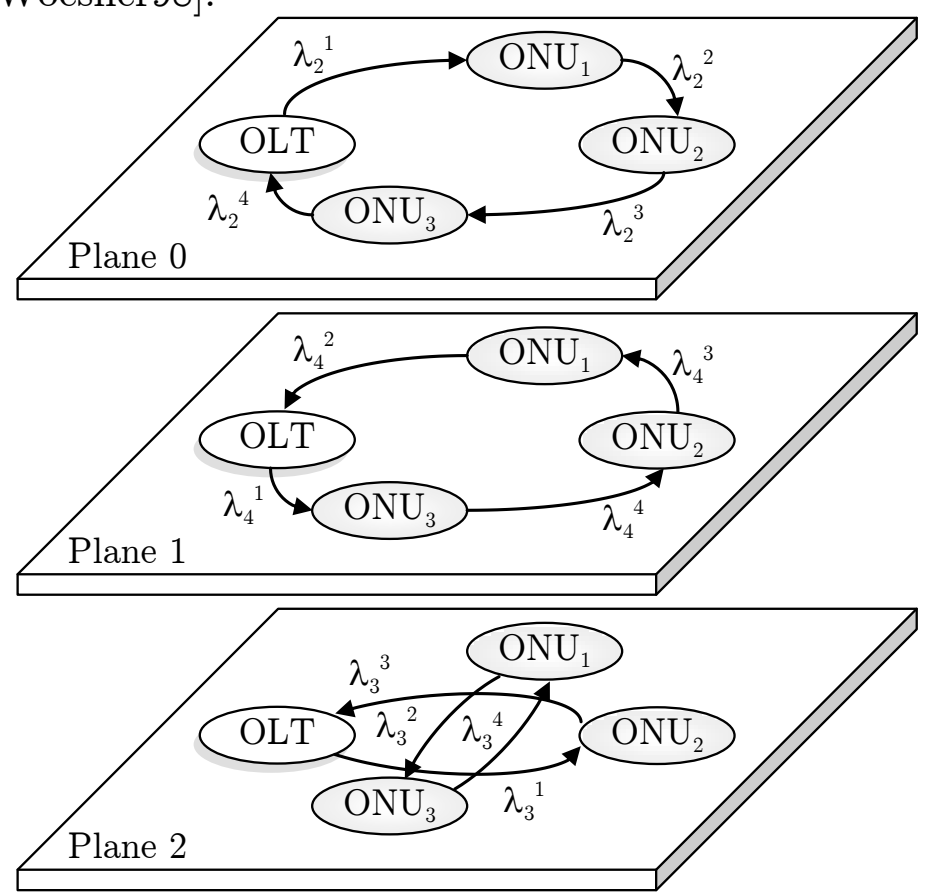

Figure 3.7: Two optical bidirectional rings formed by a

The example in Figure 3.6 physical star topology in Figure 3.6.

demonstrates the usage of another characteristic of the WR: the cyclic routing property (see section 3.1). We imagine the usage of eight consecutive wavelengths per station. Therefore, the number of used wavelengths is $2 \cdot \mathrm{M}$. As a result, the number of wavelengths that connect the stations is doubled. We obtain a similar result as we increase the coarseness factor as mentioned before.

By combining the usage of WR with coarseness factors greater than one and utilizing the cyclic routing property behavior of the WR, we are able to create optical rings with a lot of channels between the stations.

WR based on AWG cannot be produced with a size greater than $80 \times 80$ because the size corresponds with the waveguide curvature that may not lie below a critical value [Photeon05]. Therefore, the number of possible connected stations is restricted to 80 . If we suppose the usage of CWDM with a maximum of 16 wavelengths, the maximum number of wavelengths used is $80 \times 16=1280$. If we want to avoid rings that do not connect all stations, the number of wavelengths used must be a prime number. The greatest prime number which is smaller than 1280 is 1279 . For this reason, we are able to set up 1279 optical rings with a single WR.

It must be pointed out that due to the physical star topology the WR represents a single point of failure. Even though the WR is passive and robust in nature, a failure of the WR could disturb the whole network.

The optical network in Figure 3.7 illustrates a multi-hop network which is another point of view. As mentioned in section 2.1, multihop networks have many disadvantages 
related to single-hop networks in terms of simplicity and transmission delay. Thus, multi-hop networks are not pursued.

\subsection{WTM Generalization}

The calculation of the entire WDM PON with the introduced WTM is only valid if the used coarseness factor is one and the number of FSR used is also one. Therefore, a generalized WTM definition is required. That enables us to calculate WDM PONs with c>1 and \#FSR $>1$. In the following paragraphs, rules to determine the correct WTM and matrix multiplication for $\mathrm{c}>1$ and $\# \mathrm{FSR}>1$ are described and explained in detail.

An incremental approach is used to develop the generalized WTM by beginning with the first case $c>1$ and \#FSR=1 followed by case 2 with $c=1$ and \#FSR $>1$. In the last step, the generalization will be finished by a combination of case one and case two.

\section{Case $1(\mathrm{c}>1, \# \mathrm{FSR}=1)$}

For single-stage WDM PONs with WR with a coarseness factor greater than one, the WTM must be changed. We proceed from the WTM in (4.13) for $c=1$ which is displayed again in (4.16). The number of columns represents the number of wavelengths used for each input port. The number of rows is equivalent to the size of the WR. Thus, the dimension of the WTM is known and independent of the coarseness factor of the WR. From this, the new WTM must have the same dimension.

For coarseness factors greater than one, we copy the first column of the WTM c times to the following columns. We continue until the matrix has the right dimension (number of columns). An example is illustrated in (4.17) for $\mathrm{c}=2$, \#FSR=1 and $\mathrm{M}=4$.

$$
\begin{aligned}
\mathrm{WTM}_{\# \mathrm{FSR}=1, \mathrm{c}=1} & =\left[\begin{array}{cccc}
\Lambda^{1} & \Lambda^{4} & \Lambda^{3} & \Lambda^{2} \\
\Lambda^{2} & \Lambda^{1} & \Lambda^{4} & \Lambda^{3} \\
\Lambda^{3} & \Lambda^{2} & \Lambda^{4} \\
\Lambda^{4} & \Lambda^{3} & \Lambda^{2} & \Lambda^{1}
\end{array}\right] \\
\mathrm{WTM}_{\# \mathrm{FSR}=1, \mathrm{c}=2} & =\left[\begin{array}{cccc}
\Lambda^{1} & \Lambda^{1} & \Lambda^{4} & \Lambda^{4} \\
\Lambda^{2} & \Lambda^{2} & \Lambda^{1} & \Lambda^{1} \\
\Lambda^{3} & \Lambda^{3} & \Lambda^{2} & \Lambda^{2} \\
\Lambda^{4} & \Lambda^{4} & \Lambda^{3} & \Lambda^{3}
\end{array}\right]
\end{aligned}
$$

The sets of $\mathrm{N}$ symbols in the obtained WTM are arranged so that horizontally arranged elements contain the same element c times. Therefore, the obtained matrix in (4.17) represents a matrix which is not a Latin squared matrix. Thus, the matrix multiplication must be modified. We know from the standard matrix multiplication that one row of the WTM is multiplied by one column of the input matrix I, which leads to one element in the output matrix O. Due to the modified WTM matrix and the constraints defined in equation (4.11) and (4.12), it is possible to get c times elements in 
the output matrix (c times due to the coarseness factor greater than one). To solve this problem, we define a modified matrix multiplication. This modified multiplication represents a new operator and is marked with the ${ }^{*}$ symbol. For each multiplication with a row of the WTM and a column of the input matrix I, the exact activities depends on the number of the elements obtained (that is equivalent to $c$ ) in the output matrix and is defined as follows:

\begin{tabular}{|c|c|}
\hline $\begin{array}{l}\text { Number of } \\
\text { obtained elements }\end{array}$ & Activities \\
\hline 0 & None \\
\hline 1 & $\begin{array}{l}\text { Normal matrix multiplication (one row multiplied by one column } \\
\text { leads to one element in the output matrix). }\end{array}$ \\
\hline $\mathrm{n}$ & $\begin{array}{l}\text { The number of obtained elements } \mathrm{n} \text { is equivalent to the coarseness } \\
\text { factor c. All obtained elements are written in the proper row of } \\
\text { the output matrix. Obtained elements were appended to existing } \\
\text { elements. }\end{array}$ \\
\hline
\end{tabular}

The following pseudocode demonstrates the modified matrix multiplication. The multiplication is performed one element at a time.

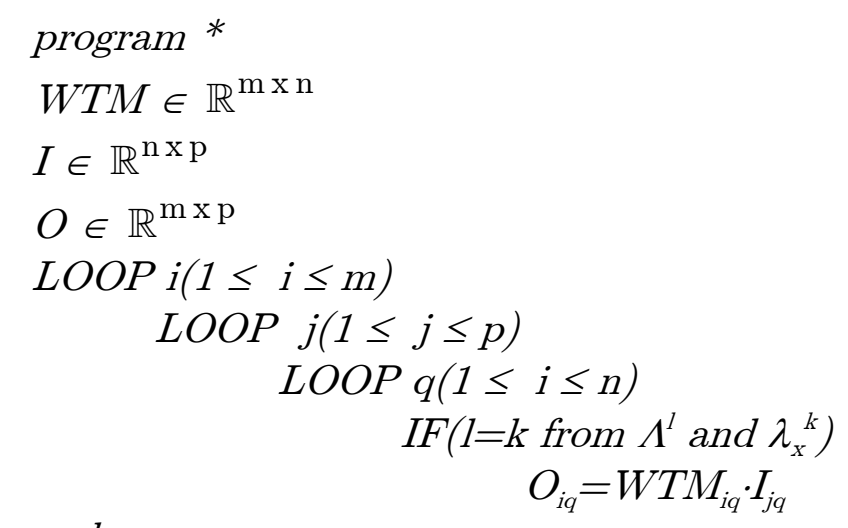

end

The example in (4.18) with an input matrix like (4.14) and a WTM with \#FSR=1 and $\mathrm{c}=2$ shows the functionality of the ${ }^{*}$ operator.

$$
\begin{aligned}
& \mathrm{O}=\mathrm{WTM}_{\# \mathrm{FSR}=1, \mathrm{c}=2} \text { * } \mathrm{I}=
\end{aligned}
$$

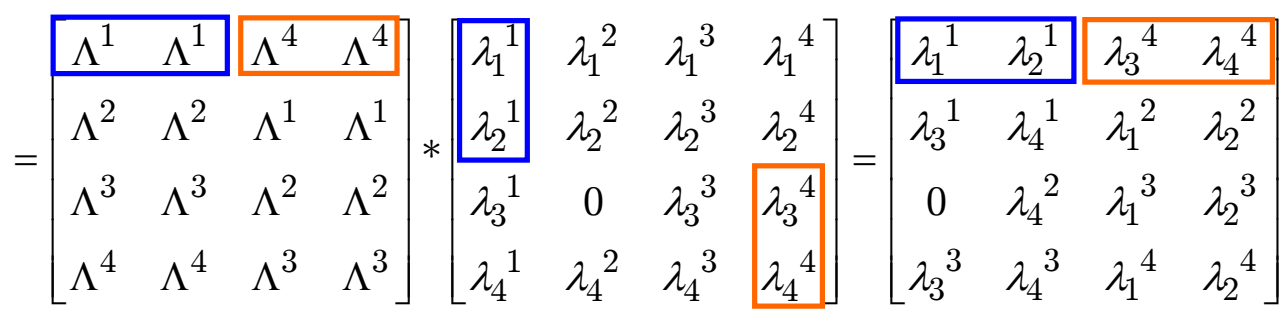

When we consider the first output port of the WR (first row of O), it appears that always two consecutive wavelengths are routed to the same output port because of the coarseness factor of two. 
Thus, we are able to calculate the WTM with coarseness factors greater than one. In the next paragraph we consider the construction of a WTM when the number of used FSR is greater than one and the coraseness factor is exactly one.

\section{Case 2 ( $c=1, \# F S R>1)$}

In this case, the WTM must be changed to fulfill the FSR enhancement. On the basis of the WTM in (4.13), the WTM must be expanded for \#FSR $>1$. The routing property of a WR is independent from the number of FSR used. On the other hand, the number of columns of the WTM is identical with the number of wavelengths used number of rows of I) and represents the number of FSR used when the channel spacing is constant. Therefore, the already known WTM in (4.13) can be extended by copying the first required part of the WTM to the second FSR and so on. An example in which four wavelengths in the first FSR and two wavelengths in the second FSR are used can be seen in (4.19).

$$
\begin{aligned}
& \mathrm{O}=\mathrm{WTM}_{\# \mathrm{FSR}=2, \mathrm{c}=1} * \mathrm{I}=
\end{aligned}
$$

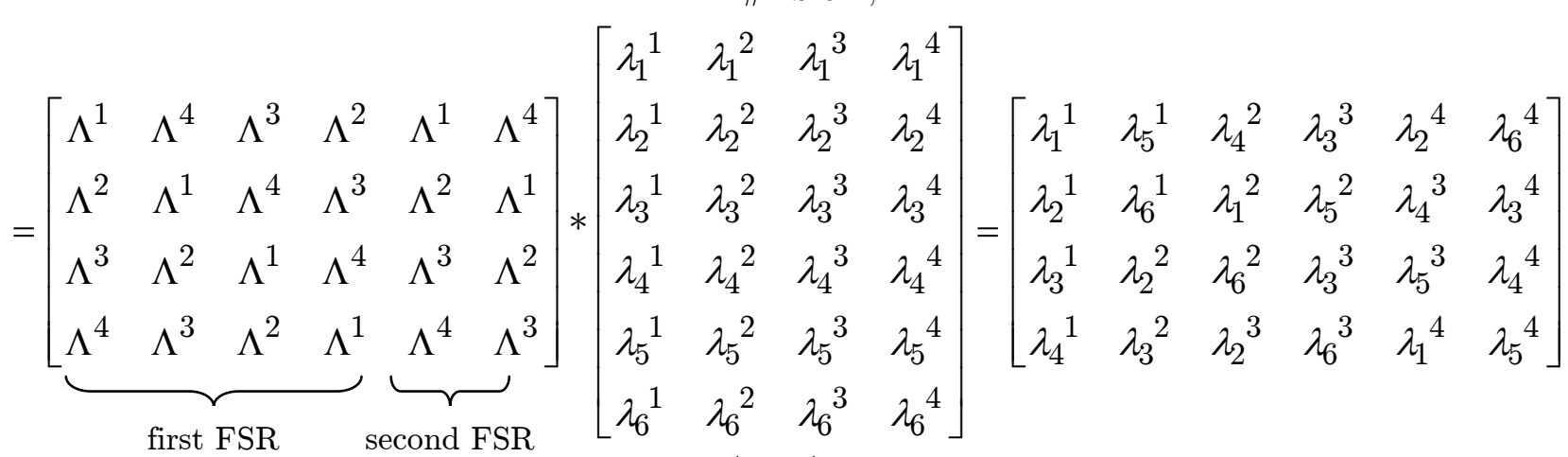

When we consider the first output port of the WR, we can realize the appearance of different wavelengths from the same input port $\left(\right.$ e.g. $\left.\lambda_{1}{ }^{1}, \lambda_{5}{ }^{1}, \ldots\right)$ due to the usage of the second FSR.

With the described methodology, we are able to construct a WTM that uses more than one FSR. This can be reached by copying columns from the first FSR to the second FSR and so on. In the next paragraph, we generalize the construction of the WTM so that we are able to calculate single-stage WDM PONs with any coarseness factors and with any number of FSR used.

\section{Case 3 (c>1 and \#FSR>1)}

The WTM with $\mathrm{c}>1$ and \#FSR $>1$ can be designed by combining both methodologies (case 1 and case 2) as explained before. The procedure to construct such a WTM is illustrated on the basis of the following example with $\mathrm{M}=4, \mathrm{n}_{\omega}=10$ and $\mathrm{c}=2$. Starting from the WTM represented in (4.19) and rewritten in (4.20), it is required to expand this WTM for $\mathrm{c}=2$. The used methodology is the same as in case 1 and can be recognized in (4.21). For simplicity, only two wavelengths are used in the second FSR. 


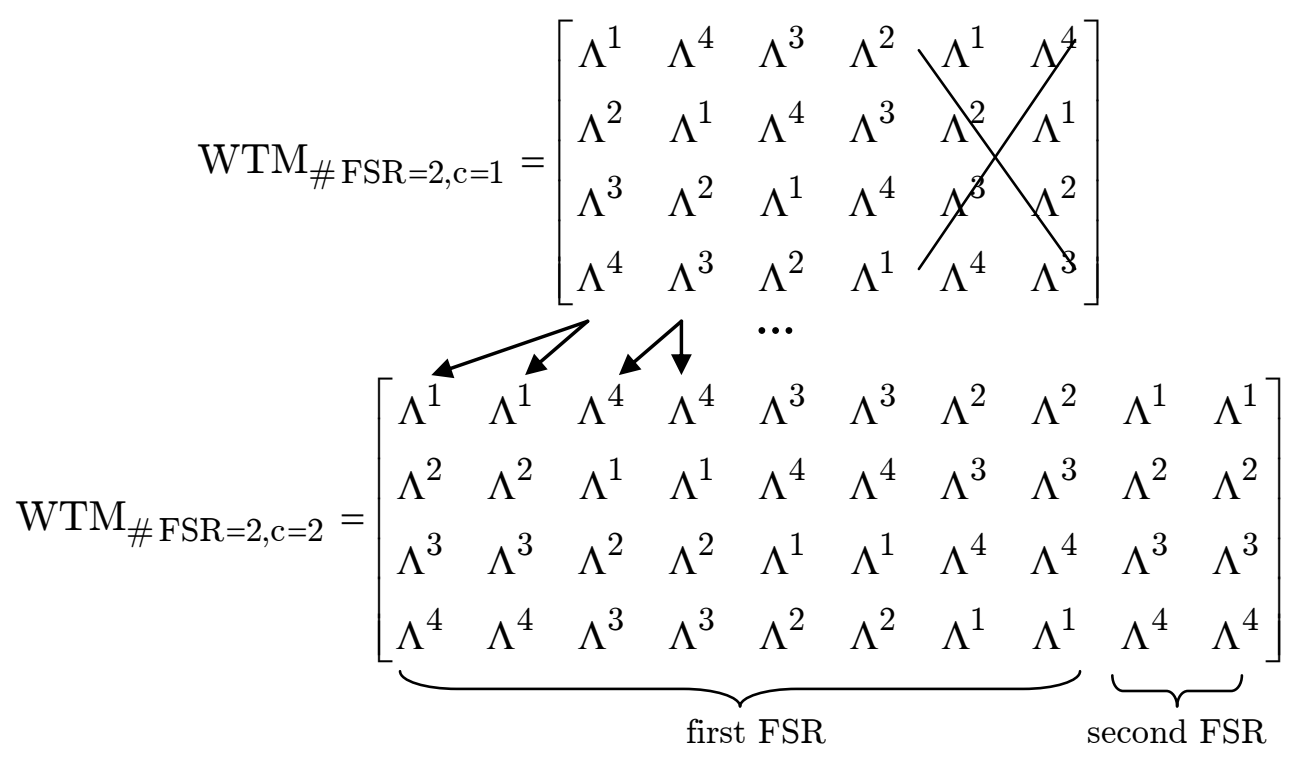

The example in (4.22) demonstrates the correct functionality for a WR with $\mathrm{M}=4, \mathrm{c}=2$, $\# \mathrm{FSR}=2$ and $\mathrm{n}_{\omega}=10$.

$$
\begin{aligned}
& \mathrm{O}=\mathrm{WTM}_{\# \mathrm{FSR}=2, \mathrm{c}=2} * \mathrm{I}= \\
& =\left[\begin{array}{cccccccccc}
\Lambda^{1} & \Lambda^{1} & \Lambda^{4} & \Lambda^{4} & \Lambda^{3} & \Lambda^{3} & \Lambda^{2} & \Lambda^{2} & \Lambda^{1} & \Lambda^{1} \\
\Lambda^{2} & \Lambda^{2} & \Lambda^{1} & \Lambda^{1} & \Lambda^{4} & \Lambda^{4} & \Lambda^{3} & \Lambda^{3} & \Lambda^{2} & \Lambda^{2} \\
\Lambda^{3} & \Lambda^{3} & \Lambda^{2} & \Lambda^{2} & \Lambda^{1} & \Lambda^{1} & \Lambda^{4} & \Lambda^{4} & \Lambda^{3} & \Lambda^{3} \\
\Lambda^{4} & \Lambda^{4} & \Lambda^{3} & \Lambda^{3} & \Lambda^{2} & \Lambda^{2} & \Lambda^{1} & \Lambda^{1} & \Lambda^{4} & \Lambda^{4}
\end{array}\right] *\left[\begin{array}{cccc}
\lambda_{1}{ }^{1} & \lambda_{1}{ }^{2} & \lambda_{1}{ }^{3} & \lambda_{1}{ }^{4} \\
\lambda_{2}{ }^{1} & \lambda_{2}{ }^{2} & \lambda_{2}{ }^{3} & \lambda_{2}{ }^{4} \\
\lambda_{3}{ }^{1} & \lambda_{3}{ }^{2} & \lambda_{3}{ }^{3} & \lambda_{3}{ }^{4} \\
\lambda_{4}{ }^{1} & \lambda_{4}{ }^{2} & \lambda_{4}{ }^{3} & \lambda_{4}{ }^{4} \\
\lambda_{5}{ }^{1} & \lambda_{5}{ }^{2} & \lambda_{5}{ }^{3} & \lambda_{5}{ }^{4} \\
\lambda_{6}{ }^{1} & \lambda_{6}{ }^{2} & \lambda_{6}{ }^{3} & \lambda_{6}{ }^{4} \\
\lambda_{7}{ }^{1} & \lambda_{7}{ }^{2} & \lambda_{7}{ }^{3} & \lambda_{7}{ }^{4} \\
\lambda_{8}{ }^{1} & \lambda_{8}{ }^{2} & \lambda_{8}{ }^{3} & \lambda_{8}{ }^{4} \\
\lambda_{9}{ }^{1} & \lambda_{9}{ }^{2} & \lambda_{9}{ }^{3} & \lambda_{9}{ }^{4} \\
\lambda_{10}{ }^{1} & \lambda_{10}{ }^{2} & \lambda_{10}{ }^{3} & \lambda_{10}{ }^{4}
\end{array}\right]= \\
& =\left[\begin{array}{llllllllll}
\lambda_{1}^{1} & \lambda_{2}{ }^{1} & \lambda_{9}{ }^{1} & \lambda_{10}{ }^{1} & \lambda_{7}^{2} & \lambda_{8}{ }^{2} & \lambda_{5}^{3} & \lambda_{6}^{3} & \lambda_{3}{ }^{4} & \lambda_{4}{ }^{4} \\
\lambda_{3}{ }^{1} & \lambda_{4}{ }^{1} & \lambda_{1}{ }^{2} & \lambda_{2}{ }^{2} & \lambda_{9}^{2} & \lambda_{10}{ }^{2} & \lambda_{7}^{3} & \lambda_{8}^{3} & \lambda_{5}{ }^{4} & \lambda_{6}{ }^{4} \\
\lambda_{5}{ }^{1} & \lambda_{6}{ }^{1} & \lambda_{3}{ }^{2} & \lambda_{4}{ }^{2} & \lambda_{1}^{3} & \lambda_{2}{ }^{3} & \lambda_{9}^{3} & \lambda_{10}{ }^{3} & \lambda_{7}{ }^{4} & \lambda_{8}{ }^{4} \\
\lambda_{7}{ }^{1} & \lambda_{8}{ }^{1} & \lambda_{5}{ }^{2} & \lambda_{6}{ }^{2} & \lambda_{3}{ }^{3} & \lambda_{4}{ }^{3} & \lambda_{1}^{4} & \lambda_{2}{ }^{4} & \lambda_{9}{ }^{4} & \lambda_{10}{ }^{4}
\end{array}\right]
\end{aligned}
$$

In the obtained output matrix, the usage of $c=2$ and the utilization of two FSRs can be seen. For simplicity, we consider only the first input port of the WR $\left(\lambda_{1}{ }^{1}-\lambda_{10}{ }^{1}\right)$. Throughout all used wavelengths, two consecutive wavelengths $\left(\lambda_{1}{ }^{1}\right.$ and $\lambda_{2}{ }^{1}, \ldots, \lambda_{9}{ }^{1}$ and $\left.\lambda_{10}{ }^{1}\right)$ appear at the same ouput port because of the coarseness factor of two. Due to the cyclic routing property of the WR, wavelengths that exceed $\mathrm{M} \cdot \mathrm{c}$ appear on the same output ports as wavelengths within M.c. For instance, $\lambda_{9}{ }^{1}$ and $\lambda_{10}{ }^{1}$ exceed $M \cdot c$ and appear equally with $\lambda_{1}{ }^{1}$ and $\lambda_{2}{ }^{1}$ at the ouput port one. 
On the basis of the generalized WTM and the definition of the new * operator, we are in the desirable position to calculate single stage WDM PONs with arbitrary size, any number of wavelengths and in consideration of the used coarseness factor. The functionality can then be calculated by a simple modified matrix multiplication, a so called * operator. From there, the next step is to determine design rules to design a single-stage WDM PON which is explained in the next section. These design rules acts as a basic for the more complicated design rules for multistage WDM PONs in chapter 4 .

\subsection{Network Design}

The objective of this section is to determine the rules to dimension single stage WDM PON networks which fulfill the paradigm shift in section 2.5. In single stage WDM PON networks, there are three potentials (see equation (4.3) and section 3.3) to comply with the paradigm shift in section 2.5 :

(1) the use of WR with a coarseness factor greater than one,

(2) the utilization of the cyclic routing property and

(3) the simultaneous usage of the possibility (1) and (2).

Starting from equation (4.3) and the inverse function of $\mathrm{a}=\mathrm{b} \bmod \mathrm{c}$ is $\mathrm{b}=\mathrm{c} \cdot \mathrm{q}+\mathrm{a}$ whereby $\mathrm{q} \in[0, \infty]$, we are able to convert equation (4.3) to $\mathrm{k}$

$$
\mathrm{k}=\mathrm{Mqc}+\mathrm{oc}-\mathrm{ic}+1
$$

With (4.23) all the discrete wavelength indices $\mathrm{k}$ can be identified on an output o. It linearly depends on the size $M$, the coarseness $\mathrm{c}$ and the number of the considered input port i. The $\mathrm{q}$ factor results from the cycling routing property and describes all the wavelengths that are routed from a certain input port to a certain output port.

In a single stage WDM PON each output port of the WR is connected to an ONU. Therefore, the number of connected users $U$ is equal to the size of the WR

$$
\mathrm{U}=\mathrm{M}
$$

The FSR can be calculated by means of equation (4.1). Because the variable $\Delta \lambda$ refers to the analog behavior of the WR, we introduce another expression to describe the FSR

$$
\mathrm{FSR}=\mathrm{M} \cdot \mathrm{c} .
$$

In a WDM PON, only a fraction of the whole FSR can be utilized. It is clear that due to the cyclic routing property of the WR, more than one FSR can be used. To express this characteristic, the number of FSR is given by 


$$
\# \mathrm{FSR}=\frac{\mathrm{n}_{\omega}}{\mathrm{FSR}}=\frac{\mathrm{n}_{\omega}}{\mathrm{M} \cdot \mathrm{c}}
$$

where $\mathrm{n}_{\omega}$ is the number of wavelengths per input fiber of the WR. It should be noted that the number of used FSR is the absolute value and can be a decimal number. When the used FSR is, for instance, half the whole FSR, then the variable \#FSR is 0.5 (independent from a possible overlapping of more than one FSR). An example in section 3.5 will demonstrate the correct usage of \#FSR.

Let us consider three examples concerning the three possible capabilities. To simplify matters, in all three examples we assume a WR with $\mathrm{M}=4$. The first one (a) uses the parameters $\mathrm{U}_{\omega}=2, \mathrm{c}=2$ and $\# \mathrm{FSR}=1$. The calculated wavelength indices $\mathrm{k}$ are shown in Table 3.1(a) and the illustration is depicted in Figure 3.8 (a). It is obvious that two consecutive wavelengths are always routed to the same output port. For simplicity, the example uses wavelengths only at the input port 1 . With this example, we can postulate the following design rule for the first possibility (1)

$$
\mathrm{U}_{\omega}=\mathrm{c} \cdot \mathrm{I} \quad \text { with } \# \mathrm{FSR}=1 \text {. }
$$

This means that if we use only one FSR, the number of wavelengths for each user $\mathrm{U}_{\omega}$ can be easily defined by using an equivalent number of $\mathrm{c}$ multiplied by the number of used inputs.

The second example (b) uses a coarseness factor of one but utilizes the cyclic routing property (in this example $\# \mathrm{FSR}=2$ ) which results also in $\mathrm{U}_{\omega}=2$. The calculated wavelength indices $\mathrm{k}$ are shown in Table 3.1(b) and are illustrated in Figure 3.8 (b). The number of wavelengths per user is equivalent as in the first example (a), but with different wavelengths. Subsequently, the following relation can be established

$$
\mathrm{U}_{\omega}=\# \mathrm{FSR} \cdot \mathrm{I} \quad \text { with } \mathrm{c}=1
$$

With equation (4.28), we are able to provide each user $\mathrm{U}_{\omega}$ times wavelengths with the WR property $\mathrm{c}=1$.

In practice, it is complicated to predefine $\mathrm{U}_{\omega}$ by using a WR with a predetermined coarseness factor or by using a certain number of used FSR. It is easier to include a combination of both options to determine $\mathrm{U}_{\omega}$. The third example (c) uses a combination of both possibilities illustrated before. The number of wavelengths routed to the same output port (is equal to the number of wavelengths per user $\mathrm{U}_{\omega}$ ) can be calculated by combining equation (4.27) and (4.28) which leads to the following design rule

$$
\mathrm{U}_{\omega}=\mathrm{c} \cdot \# \mathrm{FSR} \cdot \mathrm{I}
$$

where I represents the number of terminated input ports of the WR. Substituting (4.26) in (4.29) leads to 


$$
\mathrm{U}_{\omega}=\frac{\mathrm{n}_{\omega} \mathrm{I}}{\mathrm{M}}
$$

Equation (4.30) shows that the number of wavelengths per user is independent from the coarseness factor! However, the wavelength indices are dependent on the coarseness factor. Another point of view is that the ratio $\mathrm{I} / \mathrm{M}$ is a decimal number between 0 and 1 and therefoe $\mathrm{U}_{\omega}$ can be maximal $\mathrm{n}_{\omega}$.

The calculated wavelength indices for $\mathrm{U}_{\omega}=3$ are shown in Table 3.1(c) and are illustrated in Figure 3.8 (c). It must be pointed out that the design rules in (4.29) and (4.30) represent an upper limit relating to $\mathrm{U}_{\omega}$ because not all wavelengths within $\mathrm{U}_{\omega}$ must be used. This condition is demonstrated in the third example (c). The calculated $\mathrm{U}_{\omega}$ is 4 wavelengths (upper limit), but only 3 wavelengths are utilized.

\begin{tabular}{cccc}
\hline Output & $(\mathrm{a})$ & $(\mathrm{b})$ & $(\mathrm{c})$ \\
\hline 1 & $1 ; 2$ & $1 ; 5$ & $1 ; 2 ; 9$ \\
2 & $3 ; 4$ & $2 ; 6$ & $3 ; 4 ; 11$ \\
3 & $5 ; 6$ & $3 ; 7$ & $5 ; 6 ; 13$ \\
4 & $7 ; 8$ & $4 ; 8$ & $7 ; 8 ; 15$ \\
\hline
\end{tabular}

Table 3.1: Calculated wavelength indices for a WR with respect to $\mathrm{M}=4 ;(\mathrm{a}) \mathrm{U}_{\omega}=2, \mathrm{c}=2$, \#FSR=1; (b) $\mathrm{U}_{\omega}=2, \mathrm{c}=1, \# \mathrm{FSR}=2 ;$ (c) $\mathrm{U}_{\omega}=3, \mathrm{c}=2, \# \mathrm{FSR}=2$.

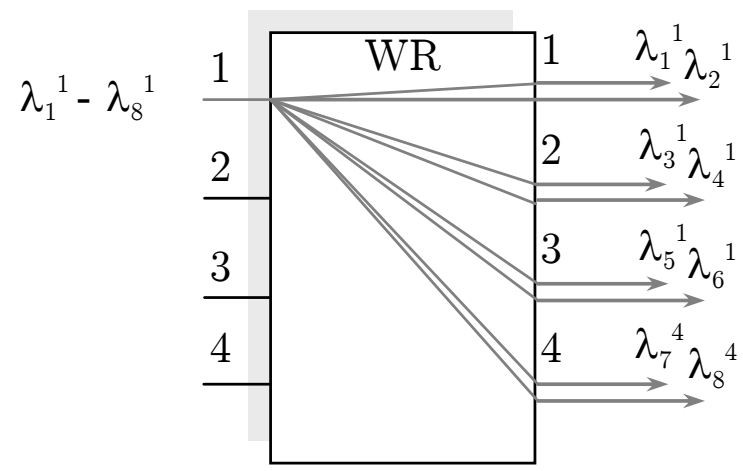

a)

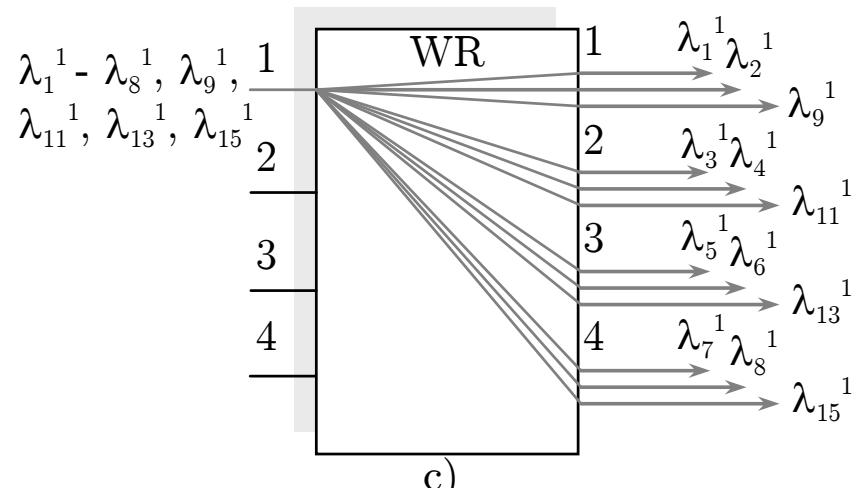

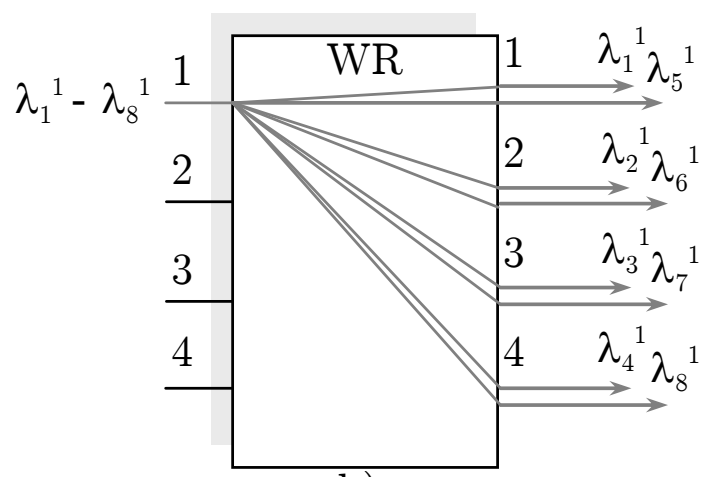

b)

Figure 3.8: WR routing example to comply with the paradigm shift in section 2.5 with respect to $\mathrm{M}=4$; (a) $\mathrm{U}_{\omega}=2, \mathrm{c}=2, \# \mathrm{FSR}=1$; (b) $\mathrm{U}_{\omega}=2, \mathrm{c}=1$, $\# \mathrm{FSR}=2 ;(\mathrm{c}) \mathrm{U}_{\omega}=3, \mathrm{c}=2, \# \mathrm{FSR}=2$.

All three examples show us an initial stage for a solution to fulfill the paradigm shift with single-stage WDM PONs. With the introduced dimensioning rules, we are able to dimension single-stage WDM PONs.

The obtained design rules act as a basis for dimensioning the more complicated multistage WDM PONs described in section 4.1. 


\subsection{Connectivity Map}

The procedure described before allows a WDM PON architecture to be designed and the parameter of each network device to be evaluated. The procedure does not allow us, however, to automatically understand which wavelength in the central office $(\mathrm{CO})$ is connected to which ONU. This connectivity map can be built manually by inspection; but for a large number of wavelengths, it is quite cumbersome.

The design of a single-stage WDM network depends on the size of the WR, the used coarseness factor, the number of used FSR and the used wavelengths. For large network sizes, it is indispensable to know which wavelength reaches which ONU.

The connectivity map can be calculated by the usage of equation (4.23) or (4.9) or with the generalized WTM in (4.21) in combination with the new introduced * operator.

The architecture in Figure 3.9 acts as an example to show the accurate utilization of the dimensioning rules and the capability to fulfill the paradigm shift with different network parameters. We assume $\mathrm{U}_{\omega}=4$ and the number of users $\mathrm{U}=16$. Utilizing equation (4.24) the size of the WR is 16 . This implies the number of required wavelengths in the whole WDM PON $\mathrm{N}_{\omega}$ with $4 \cdot 16=64$ wavelengths. The number of used fibers between the OLT and the WR can be determined as follows: referring to the CWDM technology [ITU-T G.694.2], only 16 different wavelengths can be used $\left(\mathrm{n}_{\omega}=16\right)$. Therefore, the number of required fibers I (=number of input ports used) is the total number of wavelengths divided by a maximum of 16 wavelengths per link which leads to 4 fibers between the OLT and the WR.

The last step is to identify the correct coarseness factor of the WR so that each user gets 4 wavelengths. Observing equation (4.29), the variable I is calculated, but the variables $c$ and \#FSR are not determined. Converting this equation to $\mathrm{U}_{\omega} / \mathrm{I}=\mathrm{c} \cdot \# \mathrm{FSR}$ leads to $1=\mathrm{c} \cdot \# \mathrm{FSR}$. This means that there are many possibilities to obtain $\mathrm{U}_{\omega}=4$.

Refering to the WDM PON in Figure 3.9, three different parameter variations of $1=\mathrm{c} \cdot \#$ FSR will show us the correct functionality. To receive a uniform distribution of the wavelengths at the ONUs, the input occupancy pattern of the WR is uniformly distributed. The first one (Figure 3.10) uses $\mathrm{c}=1$; therefore, \#FSR must be one. We can realize that each ONU receives four different wavelengths which always cames from the four different used input ports of the WR. The second example uses $c=2$ and from there \#FSR must be 0.5. The obtained connectivity map is illustrated in Figure 3.11. Due to $\mathrm{c}=2$, two consecutive wavelengths are always routed to the same ONU and leads to another connectivity pattern. The last example uses $\mathrm{c}=4$; thus, \#FSR=0.25. The resulted connectivity map in Figure 3.12 shows us that four different wavelengths from the same input port of the WR is always routed to the same ONU. 


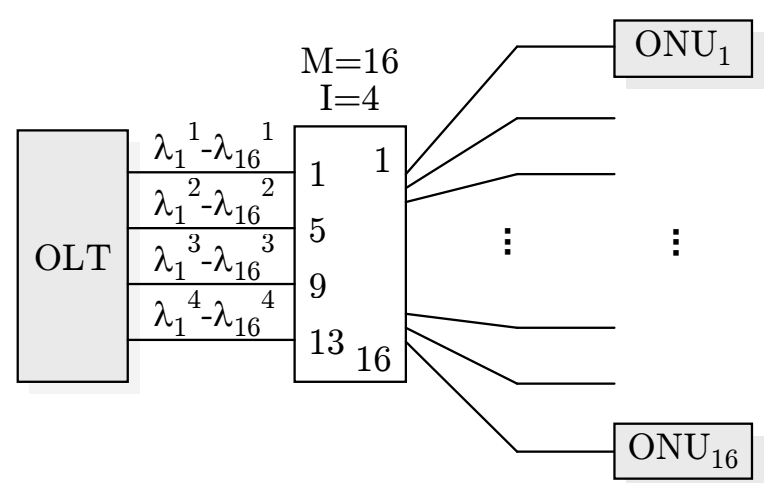

Figure 3.9: Example of a single-stage WDM PON with respect to $\mathrm{U}=16, \mathrm{M}=16, \mathrm{I}=4$ and $\mathrm{U}_{\omega}=4$.

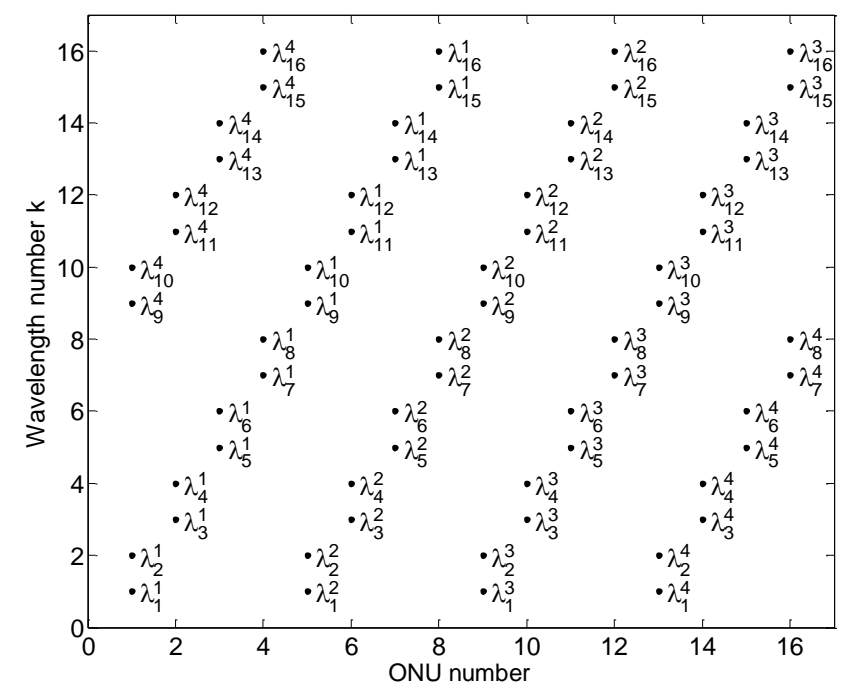

Figure 3.11: Connectivity map of the single-stage architecture from Figure 3.9 with respect to $\mathrm{c}=2$, $\# \mathrm{FSR}=0.5$ and $\mathrm{U}_{\omega}=4$.

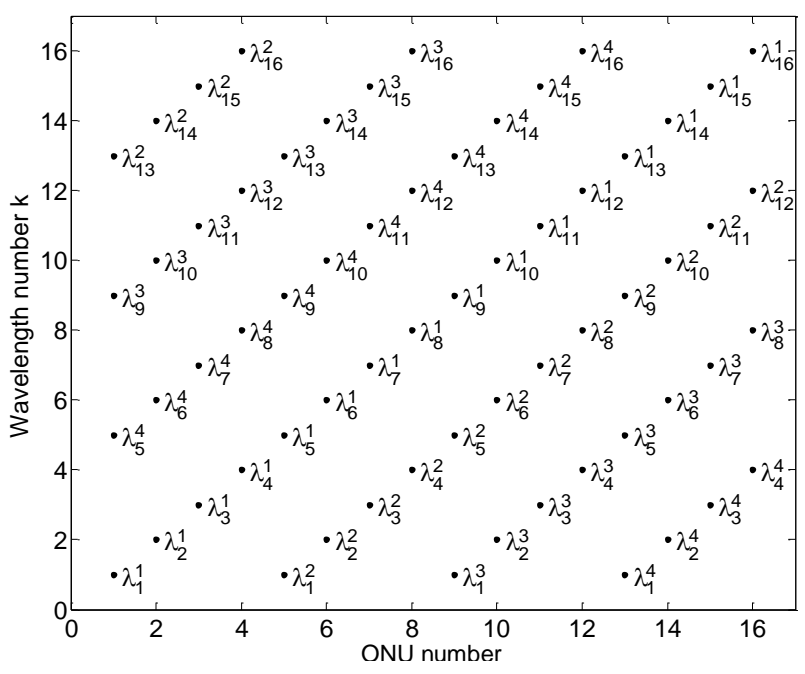

Figure 3.10: Connectivity map of the single-stage architecture from Figure 3.9 with respect to $\mathrm{c}=1$, $\# \mathrm{FSR}=1$ and $\mathrm{U}_{\omega}=4$.

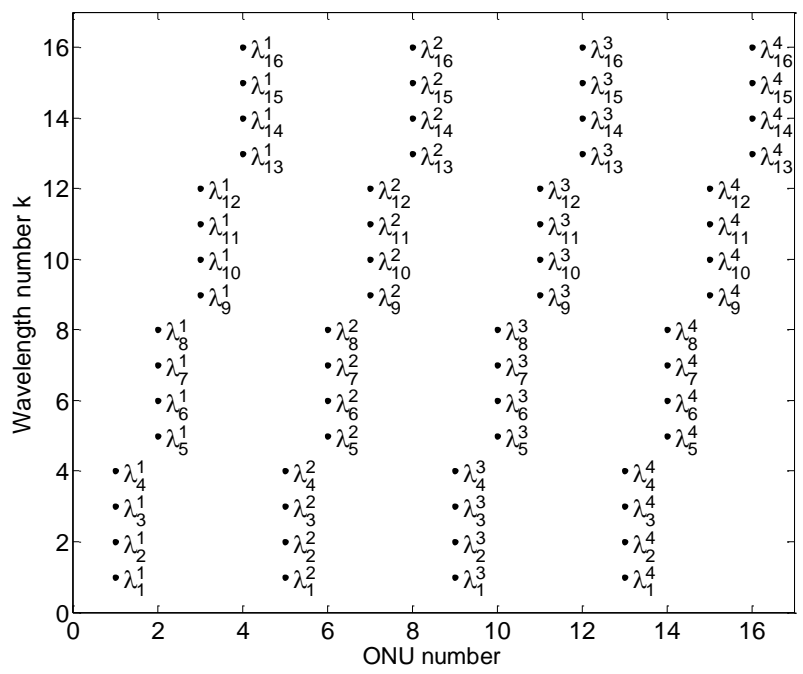

Figure 3.12: Connectivity map of the single-stage architecture from Figure 3.9 with respect to $\mathrm{c}=4$, $\# \mathrm{FSR}=0.25$ and $\mathrm{U}_{\omega}=4$. 


\subsection{Capacity Limits}

The goal of this section is to find the greatest possible network in terms of the capacity limit and the maximum number of users due to technology constraints. We define the capacity limit to be the product of the channel bitrate on each source or wavelength $\mathrm{B}_{0}$ and the number of sources or wavelengths $\mathrm{N}_{\omega}$. The number of wavelengths depends on the accessible optical spectrum $\Delta \mathrm{F}$ and the channel spacing $\Delta \mathrm{f}$ (see Figure 2.8). The capacity limit for a single-stage WDM PON is then given by

$$
\mathrm{B}_{\mathrm{N}}=\mathrm{B}_{0} \cdot \mathrm{N}_{\omega}=\mathrm{B}_{0} \cdot \mathrm{M} \cdot \frac{\Delta \mathrm{F}}{\Delta \mathrm{f}}=\mathrm{B}_{0} \cdot \mathrm{M}^{2} \cdot \mathrm{U}_{\omega}
$$

On the strength of the restricted optical spectrum of the used components, the accessible optical spectrum is limited. The channel spacing depends on the WDM technology. Typical channel spacings are 20nm [ITU-T G.694.2] for CWDM and 1.6nm, $0.8 \mathrm{~nm}, 0.4 \mathrm{~nm}, 0.2 \mathrm{~nm}$ and $0.1 \mathrm{~nm}$ for DWDM technology [ITU-T G.694.1]. The optical spectral range $\Delta \mathrm{F}$ is not limited in both ITU-T standards. If we assume an accessible optical spectrum of $\Delta \mathrm{F}=200 \mathrm{~nm}$ and a channel bitrate of $1 \mathrm{Gbit} / \mathrm{s}$, we achieve the number of wavelengths and the capacity limits can be seen in Table 3.2.

Due to technology restrictions the size of the WR and therefore the maximum number of users is limited. Today a maximum size of 80 inputs and outputs are realistic [Smit96, Photeon05] and therefore a maximum number of users of 80 .

According to the paradigm of at least one wavelength per service and user, the maximum possible number of users and therefore the maximum network structures is given. In other words, the multiplication of the number of users $\mathrm{U}$ with the number of wavelengths per user cannot exceed the number $\frac{\Delta \mathrm{F}}{\Delta \mathrm{f}} \cdot \mathrm{M}$.

\begin{tabular}{crr}
\hline $\begin{array}{c}\Delta \mathrm{f} \\
{[\mathrm{nm}]}\end{array}$ & $\mathrm{N}_{\omega}=\frac{\Delta \mathrm{F}}{\Delta \mathrm{f}} \cdot \mathrm{M}$ & $\begin{array}{c}\mathrm{B}_{\mathrm{N}} \\
{[\mathrm{Gbit} / \mathrm{s}]}\end{array}$ \\
\hline 20 & 800 & 800 \\
1.6 & 10,000 & 10,000 \\
0.8 & 20,000 & 20,000 \\
0.4 & 40,000 & 40,000 \\
0.2 & 80,000 & 80,000 \\
0.1 & 160,000 & 160,000 \\
\hline
\end{tabular}

Table 3.2: Maximum number of possible wavelengths $\mathrm{N}_{\omega}$ and the network capacity limit $B_{N}$ for different channel spacings $\Delta f$ with respect to $\Delta \mathrm{F}=200 \mathrm{~nm}, \mathrm{M}=80$ and $\mathrm{B}_{0}=1 \mathrm{Gbit} / \mathrm{s}$.

Smaller numbers of $\Delta \mathrm{f}$ results in higher capacity limits but leads to higher costs for lasers and WR. 


\subsection{Summary}

The consideration in this chapter is the basis for so-called multistage WDM PONs described in the next chapter. The main advantage of the WR is the property to reuse wavelengths (cyclic routing property) independently from the chosen input pattern. This property is responsible for the extremely high capacity of each single WR (see section $3.6)$.

In this chapter, 2 possibilities are identified to describe the functionality of a WR. The first one (equation (4.3)) enables the separate calculation of the input and output port for each wavelength. The second one is based on a WTM (see section 3.2) to make the calculation of one single WR in one step with only one standard matrix multiplication possible. Originally, the WTM is defined for WR with $\mathrm{c}=1$ and $\# \mathrm{FSR}=1$. A generalization of the WTM in combination with the introduction of a new matrix operator (based on a modified matrix multiplication) was successfully demonstrated step-by-step (section 3.3). This allows the design of a single-stage WDM PON and the adherence of the paradigm shift in section 2.5.

For a large number of wavelengths and/or number of users, it is quite cumbersome to understand which wavelength in the central office is connected to which ONU. Thus, a tool was developed to calculate which wavelength in the central office reaches which ONU. The resulting map was introduced by the phrase connectivity map.

In summary, the main drawback of a single-stage WDM PON is the limited network size in terms of number of users and the restricted expandability. In the next chapter, these limitations are eliminated by a serial connection of WRs. This type of network is called multistage WDM PON. 


\section{Multistage Networks}

multistage WDM PON uses more than one WR in serial. As a consequence, the
number of stages is greater than one compared with single-stage WDM PONs. A
similar concept based on passive-star couplers can be found in [Borella98, p. 275 et seq.]. This concept is called a multilevel optical network (MON).

A multistage WDM PON is not easy to understand and to calculate. The objective is to determine rules to calculate such networks and to build a design tool that helps to better understand the properties of multistage WDM PON networks. Furthermore, it should allow researchers the study and introduction of new architectures.

The single-stage architecture described in chapter 3 suffers from multiple limitations. These include difficulties in scaling the number of ONUs once the network is laid out. Another problem is the limited number of users due to the limitations on the WR size. Furthermore, there is a limited spectral range in the network and therefore a limited number of useable wavelengths. The implementation of the paradigm shift in section 2.5 needs many different wavelengths. Due to the cost sensitivity of access networks, channel spacings of 20nm [ITU-T G.694.2] offer the most advantages for optical access networks to use uncooled DFB Lasers [Tanis02]. Thus, only a handful of wavelengths can be used simultaneously. In this case, we need architectures that allow for the reuse of wavelengths in the same WDM PON network. Therefore, we will examine new architectures to abolish these restrictions.

Further capabilities can be achieved by optical networks equipped with wavelength conversion possibilities [Mukherjee97, p. 431 et seq., Mohammad03, p. 433 et seq.]. At the moment, wavelength conversion is highly cost-intensive. Due to the tight cost situation in access networks, wavelength conversion is not an issue in this work. 


\subsection{Network Design}

The goal of this section is to find rules to dimensional multistage WDM PONs that fulfill the paradigm shift in section 2.5 ; i.e., each user can be connected with the central office by more than one wavelength.

\subsubsection{Design Conventions}

Building multistage WDM PON networks, we need more than one WR. So we need rules on the basis of the routing properties in equation (4.3) to calculate such multistage WDM PONs. The question is how many stages are needed and what WR parameters are required in the various stages of the multistage network.

An access network is defined as the set of equipment and required infrastructure which are necessary to establish a connection between the central office (CO) and the optical network unit (ONU). Due to economical considerations, it is useful to concentrate as many users as possible on an OLT. This implies a tree topology as a useful topology, whereas the OLT is on the root. Thus, we consider only tree topologies in this work.

An essential design parameter is the number of wavelengths per user. Because of the relatively wide gaps between the optical channels, the maximum number of wavelengths is strictly limited (see section 3.1). Therefore, a frequently reuse of wavelengths are unavoidable.

For the description of the network topology, the following symbols similarly to [Maier00] are introduced:

- $\mathrm{n}_{\omega}$

- $\mathrm{U}_{\omega}$

- $\mathrm{N}_{\omega}$

$\bullet U$

- $\mathrm{S}$

- $\overrightarrow{\mathrm{N}}=\left\{\mathrm{N}_{1}, \mathrm{~N}_{2}, \ldots, \mathrm{N}_{\mathrm{S}}\right\}$

- $\overrightarrow{\mathrm{I}}=\left\{\mathrm{I}_{1}, \mathrm{I}_{2}, \ldots, \mathrm{I}_{\mathrm{S}}\right\}$

- $\overrightarrow{\mathrm{c}}=\left\{\mathrm{c}_{1}, \mathrm{c}_{2}, \ldots, \mathrm{c}_{\mathrm{S}}\right\}$

- $\overrightarrow{\mathrm{M}}=\left\{\mathrm{M}_{1}, \mathrm{M}_{2}, \ldots, \mathrm{M}_{\mathrm{S}}\right\}$

- a number of wavelengths in a fiber

number of wavelengths per user

total number of wavelengths in the entire network

number of users connected to the WDM PON

number of stages of the considered WDM PON

vector containing the number of $\mathrm{WR}$ of all $\mathrm{S}$ stages

vector containing the number of input ports used of a single WR of all $\mathrm{S}$ stages. This vector represents also the number of fibers per WR that connect stage $\mathrm{s}$ with stage $\mathrm{s}+1$

vector containing the coarseness factor of the WR of all $S$ stages

vector containing the size of the $\mathrm{WR}$ of all $\mathrm{S}$ stages

interval between terminated input ports of a WR 
Each element within the vectors $\overrightarrow{\mathrm{I}}, \overrightarrow{\mathrm{c}}$ and $\overrightarrow{\mathrm{M}}$ is constant for an entire stage. This assumption has an influence of the network architecture and is well founded as explained in the following. In the first design step, each ONU is assigned to the same number of wavelengths $\mathrm{U}_{\omega}$. As a consequence, the tree topology must be built on an evenly distributed tree. This can be achieved if the vectors $\overrightarrow{\mathrm{I}}, \overrightarrow{\mathrm{c}}$ and $\overrightarrow{\mathrm{M}}$ are constant in each stage. That will lead to simpler network architectures which are better to understand and which reduce the number of possible architectures (section 5.2) significantly.

To allow only regular and practicable topologies, the network structures considered in this work is based on the following design conventions:

(I) Each user could obtain at least one wavelength

$$
\mathrm{U}_{\omega} \geq 1
$$

(II) Due to the considered tree topology, the first stage consists of one WR device

$$
\mathrm{N}_{1}=1
$$

(III) Each output port of a stage $\mathrm{s}$ is either connected to an input port of the following stage $\mathrm{s}+1$ or to an $\mathrm{ONU}$; therefore, the number of connected users are

$$
\mathrm{U}=\mathrm{M}_{\mathrm{S}} \cdot \mathrm{N}_{\mathrm{S}}
$$

Compared with single-stage networks (equation (4.24)), the number of connected users is $\mathrm{N}_{\mathrm{s}}$ times greater.

(IV) The coarseness for the stage $\mathrm{s}$ is

$$
\mathrm{c}_{\mathrm{S}} \geq 1
$$

It would be pointless to use a WR with a coarseness factor of 0 .

(V) We observe only networks with a tree topology. The number of WR is increasing stage by stage, so

$$
\mathrm{N}_{\mathrm{S}-1}<\mathrm{N}_{\mathrm{S}} \quad \mathrm{s} \in[2, \mathrm{~S}]
$$

(VI) To accomplish the design convention (III) and to permit only networks with WR that are connected (at least by one link) to a WR from the previous stage or to the OLT in the first stage, we define

$$
\mathrm{N}_{\mathrm{s}-1} \cdot \mathrm{M}_{\mathrm{s}-1}<\mathrm{N}_{\mathrm{s}} \cdot \mathrm{M}_{\mathrm{s}}
$$


(VII) The size $\mathrm{M}$ and the coarseness factor $\mathrm{c}$ of the WR are constant for all WR in the same stage.

(VIII) For all WR, the number of input ports is equal to the number of output ports.

(IX) All ONUs connected require the same number of wavelengths $\mathrm{U}_{\omega}$.

(X) In an increased coarseness (IC) WDM PON, the coarseness factor of the first $\mathrm{WR}$ is one $\left(c_{1}=1\right)$ to receive coarseness factors in the last stage in the range of technology feasibility.

(XI) Similarly as in design convention (X), the coarseness factor in a decreased coarseness (DC) WDM PON is one in the last stage $\left(c_{S}=1\right)$.

(XII) The coarseness factor in an IC WDM PON increases stage by stage or is equal to the previous stage

$$
\mathrm{c}_{\mathrm{s}+1} \geq \mathrm{c}_{\mathrm{S}}
$$

(XIII) In a DC WDM PON, the coarseness factor decreases stage by stage or is equal to the previous stage

$$
\mathrm{c}_{\mathrm{S}} \geq \mathrm{c}_{\mathrm{s}+1}
$$




\subsubsection{Network Model}

Figure 4.1 represents the architecture to model the multistage WDM PON similarly as in [Maier00]. The WDM PON network connects a number of ONUs to a single OLT, which is typically located in the CO. In our model, the WDM PON is partitioned in stages and interstages as shown in Figure 4.1. The WR are placed at the defined stages where an interstage represents the fibers which connect the device in stage s with the device in stage $\mathrm{s}+1$. The model consists of $\mathrm{S}+1$ stages, which are connected with $\mathrm{S}+1$ interstages. In this work, we assume that all devices in all stages are solely WR. The constant interval between terminated input ports of a WR in stage $\mathrm{s}$ is the relation $\mathrm{M}_{\mathrm{s}} / \mathrm{I}_{\mathrm{s}}$ and is denoted by $\mathrm{a}_{\mathrm{s}}$. Stages and interstages are numbered in ascending order beginning with zero in the case of the first stage and interstage. Both, stages and interstages end with the index S. The number of fibers in an interstage $\mathrm{s}$ is denoted by $I_{s}$. The number of WRs in stage 1 is always 1 and is increasing stage by stage to form a tree topology. The number of ONUs U, which can be connected to the WDM PON, is equal to the number of output ports in the last stage $\mathrm{S}$ and can be calculated by design convention (III).

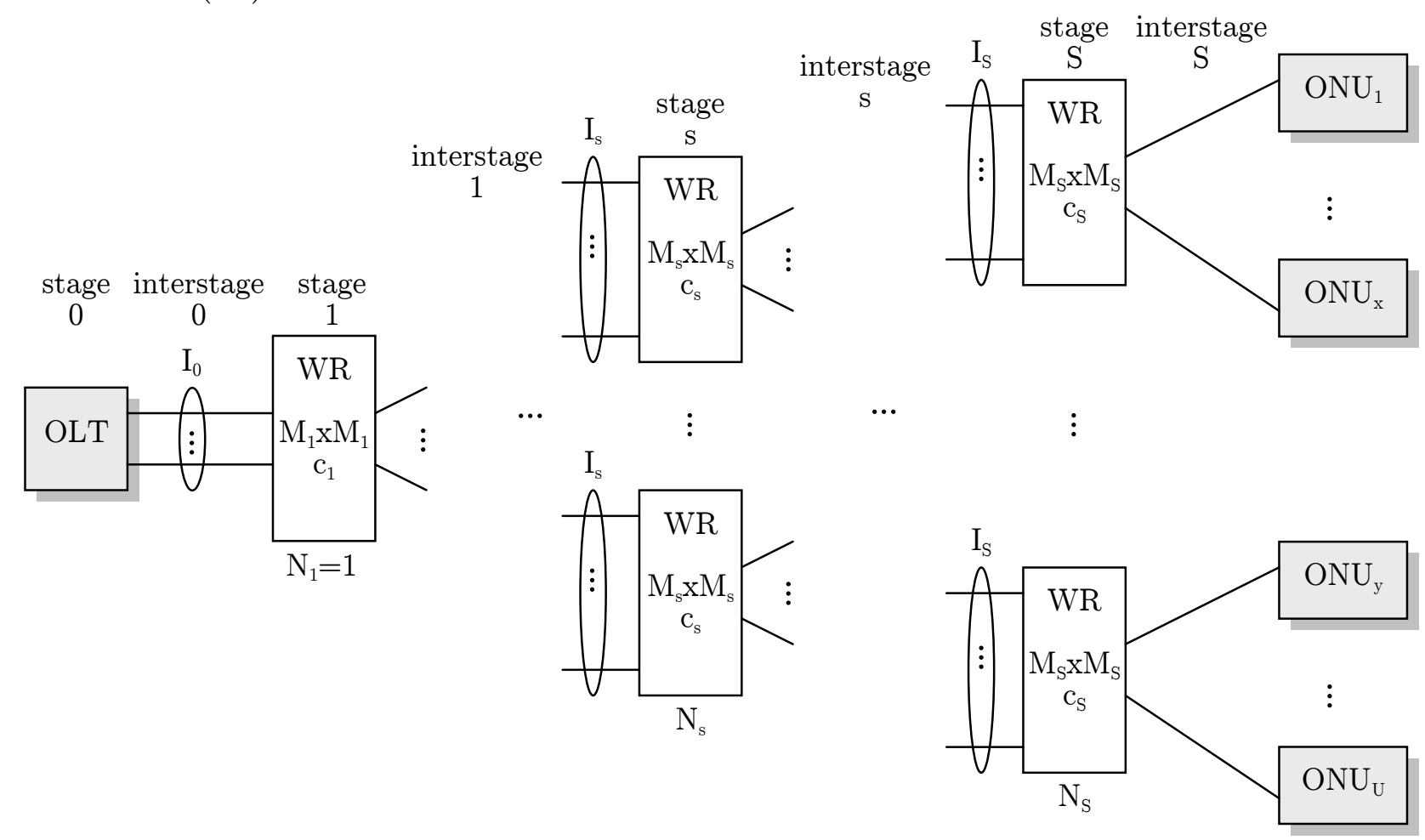

Figure 4.1: Reference architecture of the multistage WDM PON. It illustrates the stages and interstages which are used for designing the network and in the connectivity map in section 4.2.

\subsubsection{Constraints of the Design Conventions}

Additionally, constraints for network parameters must be defined and strictly adhered. The input vector I must always be greater than one. Otherwise, the involved WR is unused and is not conform to the design convention (III). A further limitation is that the input vector cannot exceed the size of the WR. Thus, the constraint is given by 


$$
1 \leq \mathrm{I}_{\mathrm{S}} \leq \mathrm{M}_{\mathrm{S}}
$$

Due to the design convention (V), we consider only networks with a tree topology. The tree topology requires a space demultiplexing in each stage to uniformly distribute all available wavelengths. In order to avoid only an exchange of wavelengths and not a space demultiplexing, the following constraint can be stated

$$
\mathrm{I}_{\mathrm{S}}<\mathrm{M}_{\mathrm{s}}
$$

The network capacity of the whole multistage WDM PON is given by

$$
\mathrm{B}_{\mathrm{N}}=\mathrm{B}_{0} \cdot \mathrm{N}_{\omega}
$$

whereas $B_{0}$ is the bitrate of a single channel and $N_{\omega}$ is the entire number of wavelengths. If our objective is to reduce cost, it is clear that all components should be working at their full capacity. In other words, we must maximize the network capacity. Otherwise, we must keep the conditions (design conventions) as mentioned before. From this it follows that a uniform distribution of the wavelengths on the output ports must be performed. This can be obtained if the size of the WR in stage $\mathrm{s}$ is a multiple of the coarseness factor in stage $\mathrm{s}$. The coarseness factor of the last stage $\mathrm{c}_{\mathrm{S}}$ determines the maximum number of consecutive wavelengths. Therefore, we define the constraint

$$
\mathrm{c}_{\mathrm{S}} \leq \mathrm{U}_{\omega}
$$

whereby the maximum number of consecutive wavelengths per ONU cannot exceed $c_{S}$.

\subsubsection{Common Design Parameters}

In this subsection, all the coarseness independent design parameters will be determined. The presented method is equivalent to a top-down approach which begins at the OLT and then goes down stage by stage.

First of all, the maximum wavelength per fiber link must be calculated. For the time being, one wavelength per ONU is assumed. From there, the following statement can be expressed: at each stage of the network, the number of users $U$ must be equal to the number of wavelengths in the whole network $\mathrm{N}_{\omega}$. At the interstage 0 , all wavelengths $\mathrm{N}_{\omega}$ are grouped in $\mathrm{I}_{0}$ fibers and must be fed all connected ONUs. Thus, the following condition holds [Maier00]

$$
\mathrm{I}_{0}=\frac{\mathrm{U}}{\mathrm{n}_{\omega}}
$$

Due to the design rule (IX), the number of wavelengths per ONU $\mathrm{U}_{\omega}$ (in interstage S) must be appointed. On the basis of the design rule (III), we consider only networks in which all output ports of the stage $\mathrm{S}$ are connected through the interstage $\mathrm{S}$ to all ONUs. Inside the multistage WDM PON, wavelengths cannot disappear and no new wavelength can be generated by reason of the passive nature of the WDM PON. The total number of used wavelengths in the whole WDM PON can be calculated by 


$$
\mathrm{N}_{\omega}=\mathrm{U} \cdot \mathrm{U}_{\omega}
$$

To perform the paradigm shift in section 2.5, we assume the number of wavelengths per ONU $\mathrm{U}_{\omega}$ according to the design rule (I) and (IX). This rule can be obtained by upgrading equation (5.13) to the following relationship

$$
\mathrm{I}_{0}=\frac{\mathrm{N}_{\omega}}{\mathrm{n}_{\omega 0}}=\frac{\mathrm{U} \cdot \mathrm{U}_{\omega}}{\mathrm{n}_{\omega 0}}
$$

in which each user obtains the same number of wavelengths.

The next step is to determine the number of required WRs in stage s. The number of output ports in stage s-1 is $\mathrm{M}_{\mathrm{s}-1} \cdot \mathrm{N}_{\mathrm{s}-1}$. Because of the design conventions (III) and (VI), we must connect all the outputs from the stage s- 1 to the stage $\mathrm{s}$, which leads to the following relation $\mathrm{N}_{\mathrm{s}} \cdot \mathrm{I}_{\mathrm{s}}=\mathrm{M}_{\mathrm{s}-1} \cdot \mathrm{N}_{\mathrm{s}-1}$. Converting it to $\mathrm{N}_{\mathrm{s}}$ leads to

$$
\mathrm{N}_{\mathrm{S}}=\frac{\mathrm{M}_{\mathrm{S}-1} \cdot \mathrm{N}_{\mathrm{S}-1}}{\mathrm{I}_{\mathrm{S}}} \quad \mathrm{s} \in[2, \mathrm{~S}]
$$

The number of required WRs in the second and third stage can be calculated using equation (5.16) and with respect to the design rule (II) using

$$
\begin{gathered}
\mathrm{N}_{2}=\frac{\mathrm{M}_{1} \cdot \mathrm{N}_{1}}{\mathrm{I}_{2}}=\frac{\mathrm{M}_{1}}{\mathrm{I}_{2}} \\
\mathrm{~N}_{3}=\frac{\mathrm{M}_{2} \cdot \mathrm{N}_{2}}{\mathrm{I}_{3}}=\frac{\mathrm{M}_{1} \cdot \mathrm{M}_{2}}{\mathrm{I}_{2} \cdot \mathrm{I}_{3}} .
\end{gathered}
$$

The general relationship for each stage can be obtained by recursion

$$
\mathrm{N}_{\mathrm{S}}=\frac{\prod_{\mathrm{x}=1}^{\mathrm{S}-1} \mathrm{M}_{\mathrm{x}}}{\prod_{\mathrm{x}=2}^{\mathrm{s}} \mathrm{I}_{\mathrm{x}}}=\frac{\mathrm{I}_{1} \prod_{\mathrm{x}=1}^{\mathrm{s}-1} \mathrm{M}_{\mathrm{x}}}{\mathrm{I}_{\mathrm{S}} \prod_{\mathrm{x}=1}^{\mathrm{s}-1} \mathrm{I}_{\mathrm{x}}} \mathrm{s} \in[2, \mathrm{~S}]
$$

Converting equation (5.16) to $\mathrm{I}_{\mathrm{s}}$ leads to the required number of input ports on stage $\mathrm{s}$

$$
\mathrm{I}_{\mathrm{S}}=\frac{\mathrm{M}_{\mathrm{S}-1} \cdot \mathrm{N}_{\mathrm{S}-1}}{\mathrm{~N}_{\mathrm{S}}} \quad \mathrm{s} \in[2, \mathrm{~S}]
$$

With the equations from (5.13) to (5.20), the coarseness independent variables $\overrightarrow{\mathrm{I}}, \mathrm{N}_{\omega}$ and $\overrightarrow{\mathrm{N}}$ can be calculated. In the next two subsections, the design dependent variables for IC and DC WDM PONs are derived. 


\subsubsection{IC WDM PON}

In an IC WDM PON, the coarseness factor increases stage by stage (see design convention XII). The primary goal is to calculate the coarseness factor in each stage so that the space demultiplexing function can be guaranteed.

In this subsection, all the coarseness dependent design parameters for IC WDM PONs will be determined. The presented method is equivalent to a top-down approach which is beginning at the OLT and then goes down stage by stage.

In Figure 4.2, a WR with the size $\mathrm{M}$ and a coarseness factor c are assumed. A comb of contiguous wavelengths is fed to each of the I input terminations. The interval between the input ports is denoted by a. Contiguous wavelengths, which are inside of the interval c, are grouped together and labeled with a $g$. All wavelengths which satisfy (4.23) appear on the output ports which display various periodical behaviors.

On the output $\mathrm{o}=1$, the first group of adjacent channels $\left(\mathrm{g}_{1}{ }^{1}\right)$ is derived from the input $\mathrm{i}=1$ and contains a number of wavelength channels given by the coarseness $\mathrm{c}$ of the WR. The second group of adjacent channels $\left(\mathrm{g}_{3}^{\mathrm{M}-\mathrm{a}}\right)$ on the output $\mathrm{o}=1$ is received from the last terminated input port M-a and is separated by an interval $a \cdot c$, with $a \cdot c=M \cdot c / I$. All wavelength packets repeat after a wavelength interval of Mc by reason of the WR cyclic routing property.

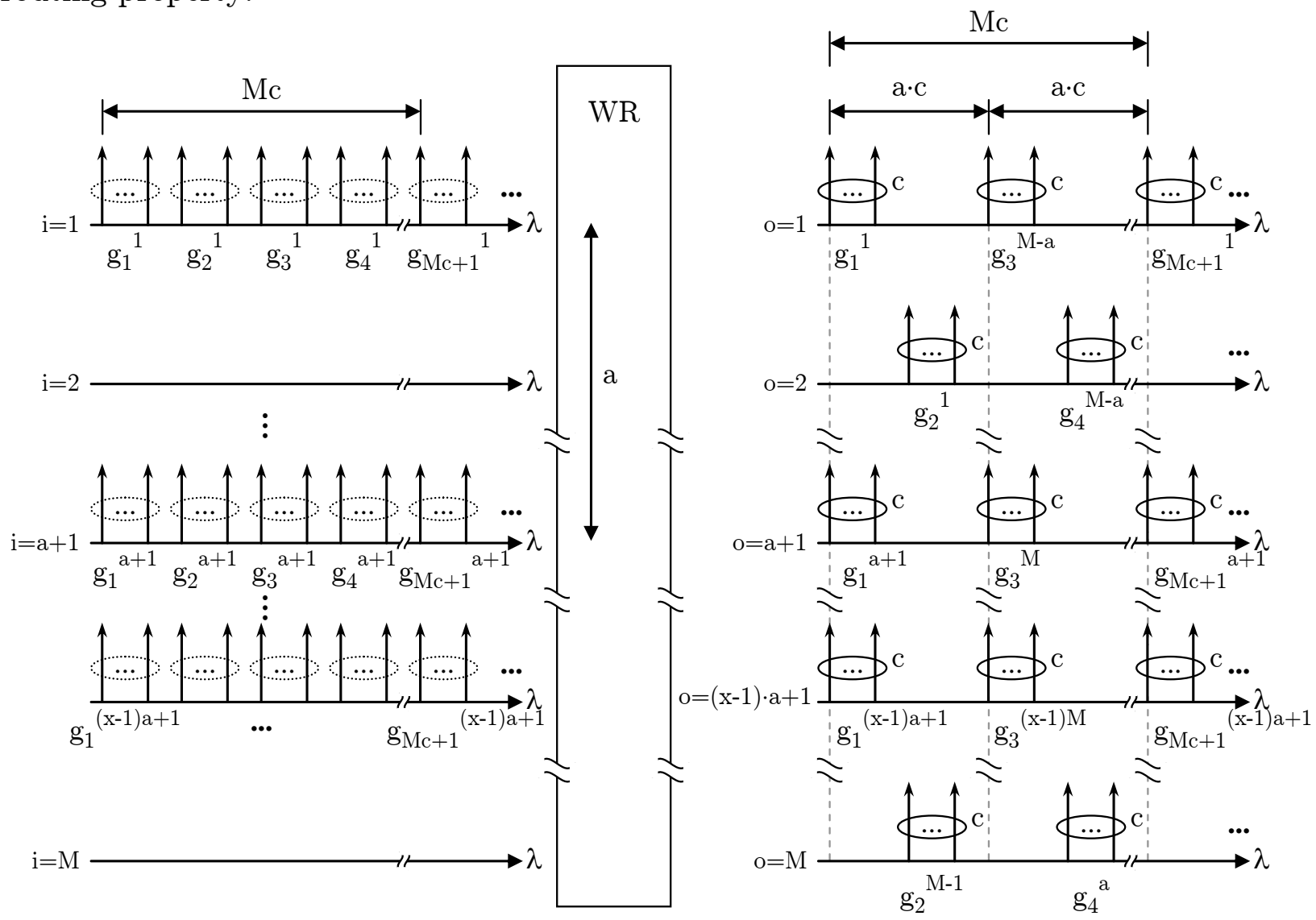

Figure 4.2: Routing example of a WR with the size M, coarseness $\mathrm{c}$ and with the interval of the input port occupancy pattern of a. 
The set of wavelengths that appears on the output port $\mathrm{o}=2$ is the set of wavelengths on the output port $\mathrm{o}=1$ shifted by a wavelength interval $\mathrm{c}$. The set of wavelengths that is routed to the output port $\mathrm{o}=\mathrm{a}+1$ is the same as the one on the output port $\mathrm{o}=1$ but with a small difference: the input ports they come from are shifted by the interval a+1 which corresponds to the interval between input ports used. In general, on the output port, which is a multiple integer of $a+1((x-1) a+1)$, the same set of wavelengths appears on the output port $a+1$ but the wavelengths received from input ports which are greater by the factor $\mathrm{x}-1$.

Two important properties can be recognized. The first one is the repeated presence of channels at the same wavelengths on different output ports. This property allows wavelength reuse and is typical for WR. It is vital that this property is independent from the choice of the input port pattern used.

It is therefore crucial to know that the second property is dependent on the choice of the input port pattern used and is called the space demultiplexing function. Consider the contiguous wavelength which is fed to the first input in Figure 4.2. This set of wavelengths is always split by the WR to different output ports. This important property allows us to build-up a multistage WDM PON with a tree-based topology as defined in the design convention (V).

In a multistage WDM PON we must guarantee that the space demultiplexing function is performed stage by stage. Thus, the inspection of the space demultiplexing function of one single WR is not sufficient. To guarantee the space demultiplexing function of each WR, the coarseness factor must be well chosen.

Let us assume that the WR in Figure 4.2 is placed in stage $\mathrm{s}$ of a WDM PON. The outputs of the WR in stage $\mathrm{s}$ are linked with the inputs of the WR in stage $\mathrm{s}+1$. All WRs in the second stage have the size $M_{s+1}$, coarseness $c_{s+1}$ and the interval between terminated input ports is $\mathrm{a}_{\mathrm{s}+1}$. In Figure 4.3 (a), the input signals in stage $\mathrm{s}+1$ are represented.

The routing performed by the WR is illustrated in Figure 4.3 (b). Let us consider the first input of the $\mathrm{WR}$ in stage $\mathrm{s}+1$. Groups of wavelengths $\left(\mathrm{g}_{1}{ }^{1}, \mathrm{~g}_{3}{ }^{1}, \ldots\right)$ are placed at a wavelength interval $a_{s} \cdot c_{s}$. All wavelengths within the interval $a_{s} \cdot c_{s}$ are already space demultiplexed in stage $\mathrm{s}$ or in previous stages. As a consequence, the coarseness factor of the WR in stage $s+1$ must be chosen equal to the interval $a_{s} \cdot c_{s}$ to ensure the space demultiplexing function in the second WR.

In Figure 4.3, the coarseness factor of the WR in stage $\mathrm{s}+1$ is chosen as follows: $\mathrm{c}_{\mathrm{s}+1}=\mathrm{a}_{\mathrm{s}} \cdot \mathrm{c}_{\mathrm{s}}$. As a result, the wavelength group $\mathrm{g}_{3}{ }^{1}$, for example, is routed to the second output port $\mathrm{O}_{\mathrm{s}+1}=2$. In other words, groups of wavelengths $\left(\mathrm{g}_{1}{ }^{1}, \mathrm{~g}_{\mathrm{Mc}+1}, \ldots\right)$ are placed at a wavelength interval $\mathrm{a}_{\mathrm{s}+1} \cdot \mathrm{c}_{\mathrm{s}+1}$. 


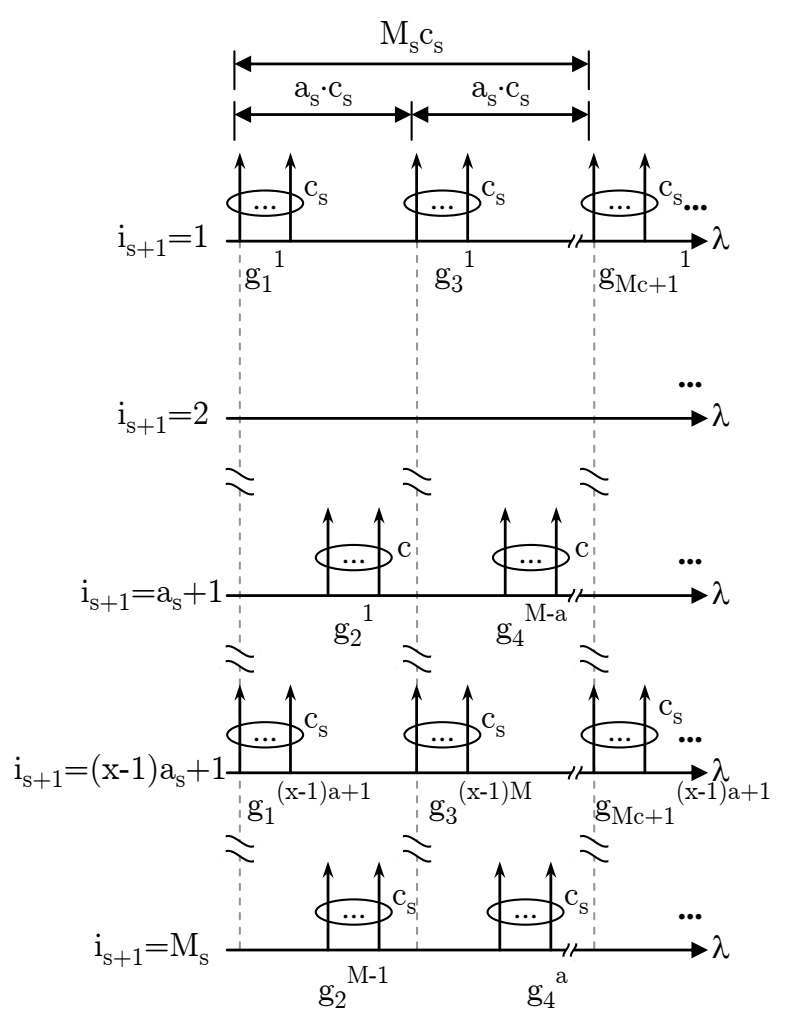

Figure 4.3: Space demultiplexing function performed by two cascaded WRs with the coarseness $c_{s}$ in stage $s$ and $c_{s+1}$ in stage $s+1$ whereby $c_{\mathrm{s}}<\mathrm{c}_{\mathrm{s}+1}$ :

(a) Input channels in stage $s+1$ resulting from the WR in stage s; we assume that the input channels are derived from the output ports of the previous stage, which can be seen in Figure 4.2; the interval between terminated input ports in stage $\mathrm{s}$ is $\mathrm{a}_{\mathrm{s}}$.

(b) Resulting output channels in stage $s+1$. The interval between input ports used is $a_{s+1}$. The coarseness $c_{s+1}$ is chosen equally to $a_{s} \cdot c_{s}$. As a consequence, the space demultiplexing function is performed in the stage $s+1$. For instance, the wavelength group $\mathrm{g}_{3}{ }^{1}$ on the input port $\mathrm{i}_{\mathrm{s}+1}=1$ is routed to the output port $\mathrm{o}_{\mathrm{s}+1}=2$.

(a)

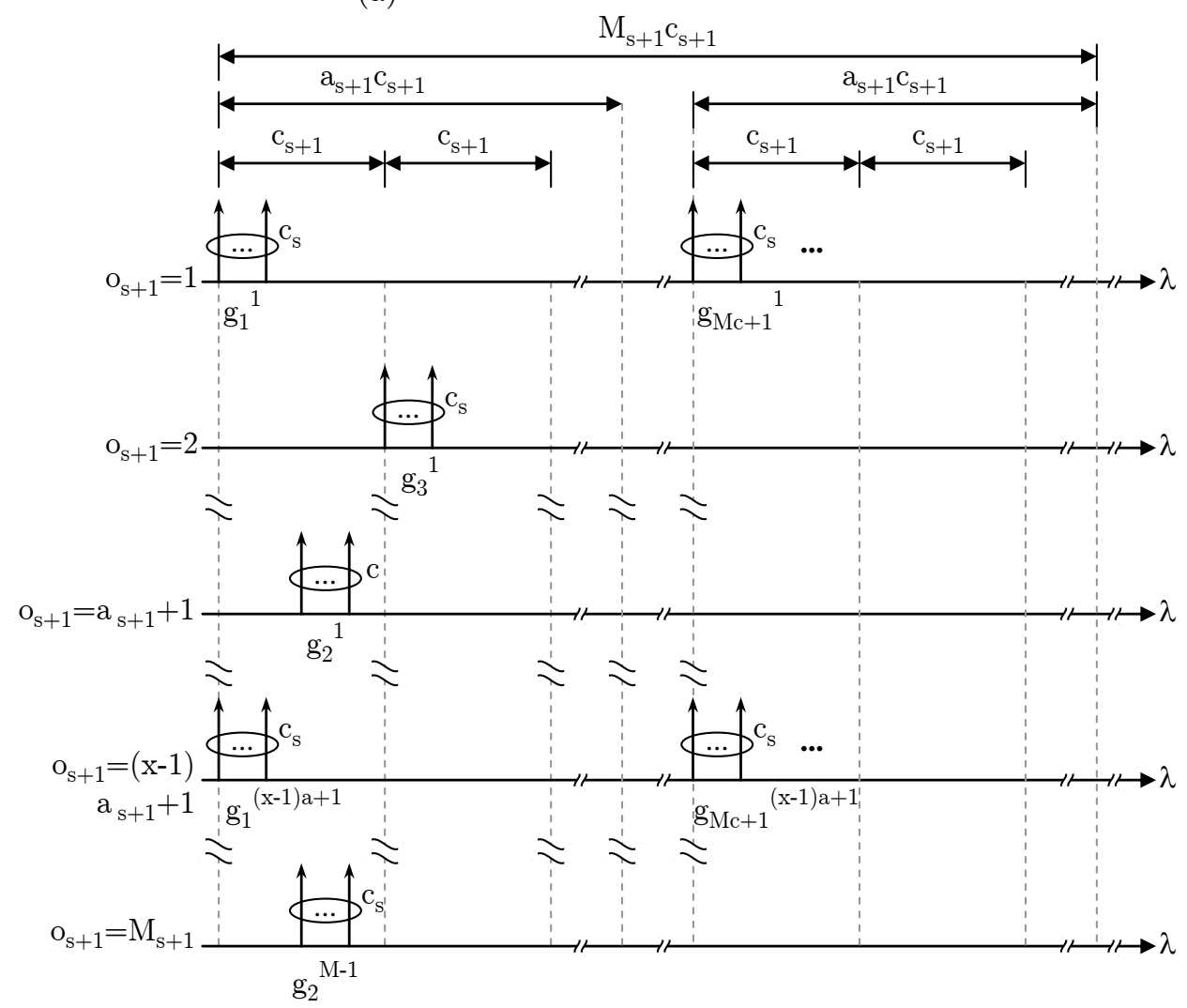

(b)

The interval of the terminated input ports of the WR in stage $s$ is $a_{s}=M_{s} / I_{s}$ and can be substituted by equation $\mathrm{c}_{\mathrm{s}+1}=\mathrm{a}_{\mathrm{s}} \cdot \mathrm{c}_{\mathrm{s}}$, which results in [Maier00]

$$
c_{\mathrm{S}+1}=\frac{\mathrm{M}_{\mathrm{S}} \cdot \mathrm{c}_{\mathrm{S}}}{\mathrm{I}_{\mathrm{S}}}
$$


Equation (5.21) allows us to recursively calculate the coarseness of the WRs in a multistage IC WDM PON. The calculation starts from the first stage and then proceeds downstream stage by stage. A drawback of equation (5.21) is that the calculation of the coarseness for a certain stage is dependent on the previous stage. In the next paragraph we try to find an equation which enables us to calculate the coarseness independently from the coarseness of the other stages.

Due to the design rule (X) and applying the condition of (5.21), the coarseness factor of the second and third stage is given by

$$
\begin{gathered}
\mathrm{c}_{2}=\frac{\mathrm{M}_{1} \cdot \mathrm{c}_{1}}{\mathrm{I}_{1}}=\frac{\mathrm{M}_{1}}{\mathrm{I}_{1}}, \\
\mathrm{c}_{3}=\frac{\mathrm{M}_{2} \cdot \mathrm{c}_{2}}{\mathrm{I}_{2}}=\frac{\mathrm{M}_{1} \cdot \mathrm{M}_{2}}{\mathrm{I}_{1} \cdot \mathrm{I}_{2}} .
\end{gathered}
$$

On the basis of equation (5.22) and (5.23), a generalization can be obtained by recursion and leads to

$$
\mathrm{c}_{\mathrm{S}}=\frac{\prod_{\mathrm{x}=1}^{\mathrm{s}-1} \mathrm{M}_{\mathrm{x}}}{\prod_{\mathrm{x}=1}^{\mathrm{s}-1} \mathrm{I}_{\mathrm{x}}} \mathrm{s} \in[2, \mathrm{~S}]
$$

By substituting equation (5.19) by (5.24), we get

$$
\mathrm{c}_{\mathrm{s}}=\frac{\mathrm{N}_{\mathrm{S}} \cdot \mathrm{I}_{\mathrm{S}}}{\mathrm{I}_{1}} \quad \mathrm{~s} \in[2, \mathrm{~S}] .
$$

The above equation can be substituted by equation (5.20), and we obtain

$$
\mathrm{c}_{\mathrm{S}}=\frac{\mathrm{N}_{\mathrm{S}-1} \cdot \mathrm{M}_{\mathrm{S}-1}}{\mathrm{I}_{1}} \quad \mathrm{~s} \in[2, \mathrm{~S}] .
$$

With the equation above, we are in the desirable position to calculate the coarseness factor independent from the coarseness of other stages.

It must be mentioned that in an IC WDM PON, we begin with a coarseness factor of one $\left(c_{1}=1\right)$ to realize technological feasible coarseness factors in the last stage. 


\subsubsection{WDM PON}

In a DC WDM PON, the coarseness decreases stage by stage. In this subsection, all the coarseness dependent design parameters will be determined. The presented method is equivalent to a bottom-up approach which begins at the ONUs and then goes up stage by stage in direction to the OLT.

Let us consider two cascaded WRs in a multistage DC WDM PON configuration. In Figure 4.4, the input and output ports of both WRs are shown in the discrete wavelength domain. The first $\mathrm{WR}$ is placed at the stage $\mathrm{s}$ and the second one at the stage $\mathrm{s}+1$. We assume that the size of the WR in stage $\mathrm{s}$ is equal to the size of the WR in stage $\mathrm{s}+1$. For simplicity, a wavelength comb $\left(\mathrm{g}_{1}{ }^{1}-\mathrm{g}_{8}{ }^{1}\right)$ can be observed (Figure 4.4 (a)), only at the first input port of the WR in stage s. The WR in stage s has the size $\mathrm{M}_{\mathrm{s}}$, the coarseness $\mathrm{c}_{\mathrm{s}}$ and an interval between terminated input ports of $\mathrm{a}_{\mathrm{s}}$.

The outputs of the WR in stage $\mathrm{s}$ are linked with the inputs of the WR in stage $\mathrm{s}+1$. The WR in the second stage has the size $\mathrm{M}_{\mathrm{s}+1}$, coarseness $\mathrm{c}_{\mathrm{s}+1}$, and the interval between terminated input ports are $\mathrm{a}_{\mathrm{s}+1}$. In Figure 4.4 (b), the input signals in stage $\mathrm{s}+1$ are represented. It must be stated that the interval between terminated input ports of the WR in stage $\mathrm{s}+1$ is assumed with 2 .

On the input $i_{s+1}=1$, the first group of adjacent channels $\left(g_{1}{ }^{1}, g_{2}{ }^{1}\right)$ comes from the input $\mathrm{i}_{\mathrm{s}}=1$ and contains a number of wavelength channels given by the coarseness $\mathrm{c}_{\mathrm{s}}$ of the WR. The second group of adjacent channels $\left(\mathrm{g}_{5}^{\mathrm{M}-\mathrm{a}}, \mathrm{g}_{6}{ }^{\mathrm{M}-\mathrm{a}}\right)$ on the input $\mathrm{i}_{\mathrm{s}+1}=3$ comes from the last terminated input port $M-a$ and is separated by an interval $M_{s} \cdot c_{s} / a_{s}$, which is equal to $I_{s} \cdot c_{s}$. All wavelength packets repeat after a wavelength interval of $M_{s} \cdot c_{s}$ by reason of the WR cyclic routing property.

In Figure 4.4 (c), the output ports of the WR in stage $\mathrm{s}+1$ are shown. Two inevitably necessary requirements can be recognized. The first one is the required smaller coarseness factor $\mathrm{c}_{\mathrm{s}+1}$, compared with $\mathrm{c}_{\mathrm{s}}$, to build a DC WDM PON. It leads to a further partitioning of the set of wavelengths. If we consider the first set of wavelengths that reach the first input port of the $\mathrm{WR}$ in stage $\mathrm{s}+1$ (Figure $4.4(\mathrm{~b})$ ), this set is determined by the coarseness $c_{\mathrm{s}}$. Due to the requirement $\mathrm{c}_{\mathrm{s}+1}<\mathrm{c}_{\mathrm{s}}$, this set of wavelengths is split into the output ports $\mathrm{O}_{\mathrm{s}+1}=1$ and $\mathrm{O}_{\mathrm{s}+1}=2$ (Figure 4.4 (c)). To guarantee the space demultiplexing function, the second requirement is that the FSR in stage $\mathrm{s}+1$ must be equal to $\mathrm{M}_{\mathrm{s}} \cdot \mathrm{c}_{\mathrm{s}} / \mathrm{a}_{\mathrm{s}}$ (Figure $4.4(\mathrm{~b})$ and $(\mathrm{c})$ ).

To guarantee the space demultiplexing function of each WR, the coarseness factor must be well chosen. From the considerations above, we can formulate the requirement to guarantee the space demultiplexing function

$$
\frac{\mathrm{M}_{\mathrm{S}} \cdot \mathrm{c}_{\mathrm{s}}}{\mathrm{a}_{\mathrm{S}}}=\mathrm{M}_{\mathrm{s}+1} \cdot \mathrm{c}_{\mathrm{s}+1}
$$




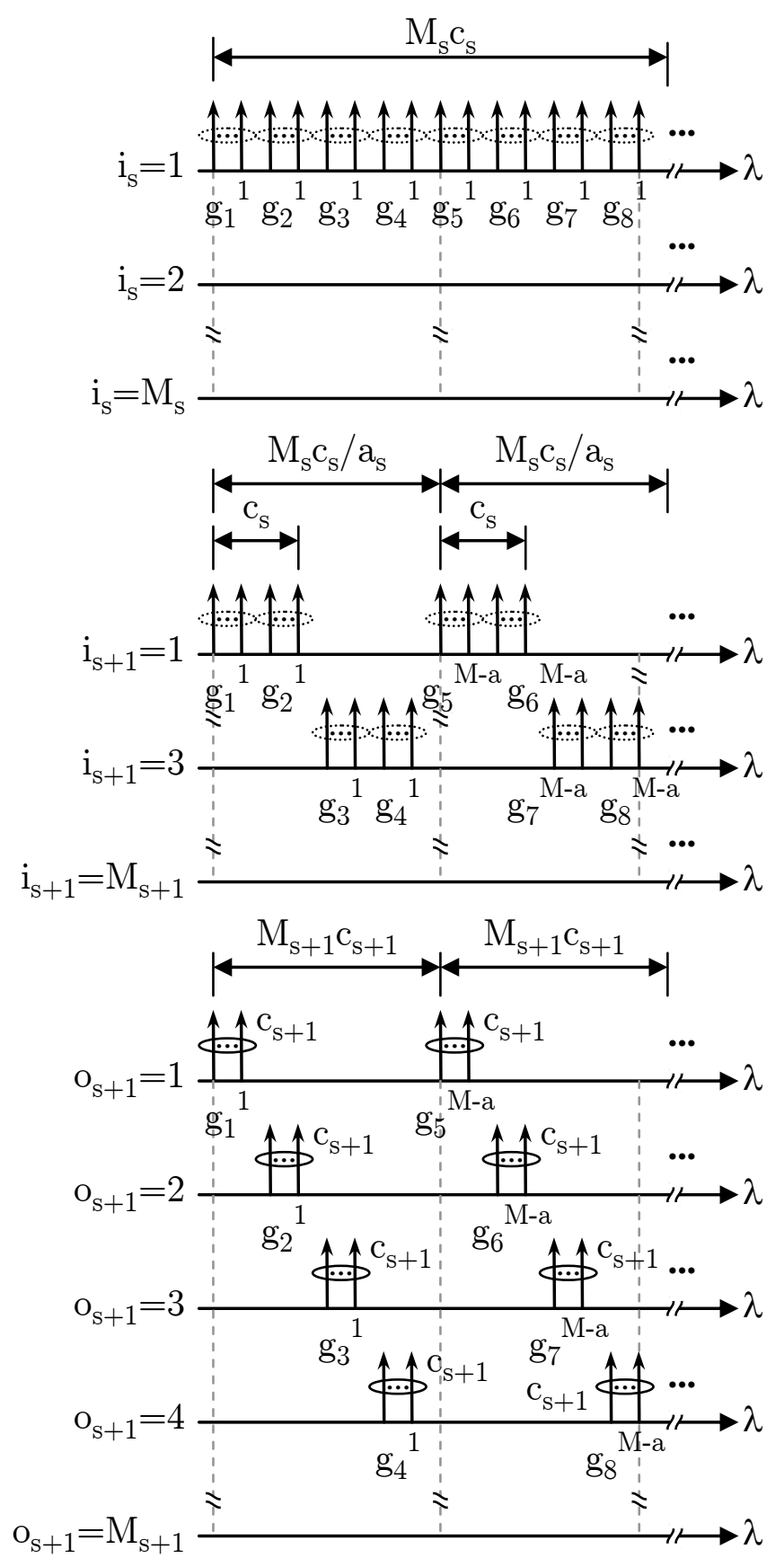

Figure 4.4: Space demultiplexing function performed by two cascaded WR in a multistage DC WDM PON with the coarseness $c_{\mathrm{s}}$ in stage $\mathrm{s}$ and $\mathrm{c}_{\mathrm{s}+1}$ in stage $s+1$ whereby $c_{s}>c_{s+1}$ :

(a)

(a) set of wavelengths at the input port in stage s;

(b) set of wavelengths at the input port of the stage $\mathrm{s}+1$;

(c) set of wavelengths at the output port of the stage $\mathrm{s}+1$. The coarseness $\mathrm{c}_{\mathrm{s}+1}$ is chosen equally to the relation $\mathrm{M}_{\mathrm{s}} \cdot \mathrm{c}_{\mathrm{s}} / \mathrm{a}_{\mathrm{s}}=\mathrm{M}_{\mathrm{s}+\mathrm{a}} \cdot \mathrm{c}_{\mathrm{s}+1}$. As a consequence, the space demultiplexing function is (b) performed in the stage $s+1$.

The term $\mathrm{a}_{\mathrm{s}}$ is equal to $\mathrm{M}_{\mathrm{s}} / \mathrm{I}_{\mathrm{s}}$ and can be substituted in equation (5.27), and results in

$$
\mathrm{c}_{\mathrm{S}}=\frac{\mathrm{M}_{\mathrm{S}+1} \cdot \mathrm{c}_{\mathrm{S}+1}}{\mathrm{I}_{\mathrm{S}}}
$$

Equation (5.28) allows us to recursively calculate the coarseness of the WRs in a multistage DC WDM PON. The calculation starts at the last stage and then goes upstream stage by stage. A drawback of equation (5.28) is that the calculation of the coarseness for a certain stage is dependent on the next stage. In the next paragraph we try to find an equation which allows us to calculate the coarseness independently from the coarseness of the other stages. 
Due to the design rule (XI) and in applying the condition of (5.28), the coarseness factor of the $\mathrm{S}-1^{\text {th }}$ and $\mathrm{S}-2^{\text {th }}$ stage is given by

$$
\begin{gathered}
\mathrm{c}_{\mathrm{S}-1}=\frac{\mathrm{M}_{\mathrm{S}} \cdot \mathrm{c}_{\mathrm{S}}}{\mathrm{I}_{\mathrm{S}-1}}=\frac{\mathrm{M}_{\mathrm{S}}}{\mathrm{I}_{\mathrm{S}-1}} \\
\mathrm{c}_{\mathrm{S}-2}=\frac{\mathrm{M}_{\mathrm{S}-1} \cdot \mathrm{c}_{\mathrm{S}-1}}{\mathrm{I}_{\mathrm{S}-2}}=\frac{\mathrm{M}_{\mathrm{S}} \cdot \mathrm{M}_{\mathrm{S}-1}}{\mathrm{I}_{\mathrm{S}-1} \cdot \mathrm{I}_{\mathrm{S}-2}} \\
\mathrm{c}_{1}=\mathrm{c}_{\mathrm{S}-\mathrm{S}+1}=\frac{\mathrm{M}_{\mathrm{S}} \cdot \mathrm{M}_{\mathrm{S}-1} \cdot \ldots \cdot \mathrm{M}_{\mathrm{S}-\mathrm{S}+2}}{\mathrm{I}_{\mathrm{S}-1} \cdot \mathrm{I}_{\mathrm{S}-2} \cdot \ldots \cdot \mathrm{I}_{\mathrm{S}-\mathrm{S}+1}}
\end{gathered}
$$

On the basis of the equations (5.29) to (5.31), a generalization can be obtained by recursion and leads to

$$
\mathrm{c}_{\mathrm{S}}=\frac{\prod_{\mathrm{x}=2}^{\mathrm{s}+1} \mathrm{M}_{\mathrm{x}}}{\prod_{\mathrm{x}=1}^{\mathrm{s}} \mathrm{I}_{\mathrm{x}}} \mathrm{s} \in[1, \mathrm{~S}-1]
$$

The indices of the product sign in equation (5.32) can be modified and we obtain

$$
\begin{gathered}
\mathrm{N}_{\mathrm{s}}=\frac{\prod_{\mathrm{x}=1}^{\mathrm{s}-1} \mathrm{M}_{\mathrm{x}}}{\prod_{\mathrm{x}=2}^{\mathrm{s}} \mathrm{I}_{\mathrm{x}}}=\frac{\mathrm{M}_{1} \cdot \mathrm{M}_{2} \cdot \ldots \cdot \mathrm{M}_{\mathrm{S}-1}}{\mathrm{I}_{2} \cdot \mathrm{I}_{3} \cdot \ldots \cdot \mathrm{I}_{\mathrm{s}-1} \cdot \mathrm{I}_{\mathrm{s}}}=\frac{\mathrm{I}_{1} \cdot \mathrm{M}_{1} \cdot \mathrm{M}_{2} \cdot \ldots \cdot \mathrm{M}_{\mathrm{S}-1} \cdot \mathrm{M}_{\mathrm{S}} \cdot \mathrm{M}_{\mathrm{S}+1}}{\mathrm{I}_{1} \cdot \mathrm{I}_{2} \cdot \mathrm{I}_{3} \cdot \ldots \cdot \mathrm{I}_{\mathrm{S}-1} \cdot \mathrm{I}_{\mathrm{s}} \cdot \mathrm{M}_{\mathrm{s}} \cdot \mathrm{M}_{\mathrm{S}+1}} \quad \mathrm{~s} \in[2, \mathrm{~S}](5) \\
\mathrm{N}_{\mathrm{s}}=\frac{\mathrm{I}_{1} \cdot \mathrm{M}_{1} \prod_{\mathrm{x}=2}^{\mathrm{s}+1} \mathrm{M}_{\mathrm{x}}}{\mathrm{M}_{\mathrm{S}} \cdot \mathrm{M}_{\mathrm{S}+1} \prod_{\mathrm{x}=1}^{\mathrm{s}} \mathrm{I}_{\mathrm{x}}} \mathrm{s} \in[2, \mathrm{~S}]
\end{gathered}
$$

Substituting equation (5.34) by (5.32) leads to

$$
\mathrm{c}_{\mathrm{s}}=\frac{\mathrm{M}_{\mathrm{S}} \cdot \mathrm{M}_{\mathrm{S}+1} \cdot \mathrm{N}_{\mathrm{S}}}{\mathrm{I}_{1} \cdot \mathrm{M}_{1}} .
$$

With the equation above, we are in the desirable position to calculate the coarseness factor independently from the coarseness of other stages. It must be stated that in a DC WDM PON, we begin with a coarseness factor of one in the last stage $\left(c_{S}=1\right)$ to realize technology feasible coarseness factors in the first stage. 


\subsubsection{Number of wavelengths per user}

An essential parameter during the design process is the number of wavelengths per user. In a single-stage WDM PON, we are able to calculate the number of wavelengths per user with equations (4.29) and (4.30). In a multistage WDM PON, equations (4.29) and (4.30) are only valid for one stage and can be used to calculate $\mathrm{U}_{\omega}$ stage by stage. It is clear that in the last stage the number of wavelengths per fiber is identical with the number of wavelengths per user. Due to the constraint in equation (5.10), the number of wavelengths per fiber decreases stage by stage.

It is important to know that equations (4.29) and (4.30) can reduce the number of wavelengths per fiber but they need not reduce it. Equation (4.29) and (4.30) reduce the number of wavelengths in stage s only if $\mathrm{U}_{\omega}<\mathrm{n}_{\omega}$, or seen from another point of view, I/M is less than 1.

It follows that the number of wavelengths per user is the minimum of all $U_{\omega}$ over all $S$ stages:

$$
\mathrm{U}_{\omega}=\min \left\{\mathrm{U}_{\omega 1}, \mathrm{U}_{\omega 2}, \ldots, \mathrm{U}_{\omega \mathrm{S}}\right\}
$$

It must be noticed that the calculation of $\mathrm{U}_{\omega}$ is independent from the coarseness factor. Thus, the presented method can be used in an IC and DC WDM PON.

\subsubsection{IC versus DC WDM PONs}

An essential property of a WDM PON is the ability to expand the network. This property can be further classified to expand the network in terms of channels per user or in terms of number of users.

To expand the network in terms of the number of wavelengths per user, it is possible to increase the demand without modifying the network. This can be done by adding new wavelengths at the $\mathrm{CO}$. This is possible thanks to the cyclic routing property of the WR, which was illustrated in detail in the previous subsections. The expansion can be done in an IC WDM PON as well as in a DC WDM PON network.

To expand the network in terms of number of users is not an easy task. In any case, the network must be changed. At the best, only one WR in the last stage must be exchanged by a WR of a larger size. It is possible to change other parameters of the larger WR to get the same functionality. Naturally, new suitable wavelengths at the CO must be added to reach the newly installed users. As a last resort each stage must be changed either in terms of larger WRs or in terms of new WRs to set up a new network tree.

Observing the expansion of the network in detail requires a differentiation between WDM PON with IC and DC characterization. 


\section{IC WDM PON}

The expansion of the network in terms of number of users requires only modifications in the last stage, which connects the new users. It is clear that at the CO the set of wavelengths must be extended with suitable wavelengths for the new users. The modification of an already installed IC WDM PON can be preferred to a DC WDM PON. This can be understood by observing equation (5.21) that characterizes an IC WDM PON. Accordingly, the size of the WR in the next stage $\left(\mathrm{M}_{\mathrm{s}+1}\right)$ does not appear. It seems that it is possible to change the size of a downstream WR without modifying other parameters. This is quite deceptive due to the fact that the number of wavelengths per user is a basic condition and can be realized by changing the parameters c and \#FSR (see section 3.4). But the number of FSRs used is unaccounted in equation (5.21) and therefore equation (5.21) is necessary but not sufficient.

The number of wavelengths per user is a basic condition and can be calculated with equation (5.36) and (4.30). Thus, it appears that the number of FSRs used and therefore the size of the WR in the last stage affect the number of used wavelengths if \#FSR>1. In other words, if the condition \#FSR>1 holds, the size of the WR can be changed but wavelengths at the CO must be changed. This is not an easy task in practice.

\section{WDM PON}

Similar to an IC WDM PON, the enlargement of the network in terms of number of users requires a modification of the last stage to connect the new users. It is easy to realize that at the $\mathrm{CO}$ the set of wavelengths must be expanded with convenient wavelengths for the new users.

The modification of an already installed DC WDM PON is not a simple task and can be understood by observing equation (5.28). A closer inspection of it shows the occurrence of the term $\mathrm{M}_{\mathrm{s}+1}$. This means that the modification of the size of the last stage influences all previous stages. As a consequence, the entire DC WDM PON must be changed if the number of users must be expanded.

Similar to an IC WDM PON, changing the size of the WR in the last stage changes the parameter \#FSR and possibly the important design parameter number of wavelengths per user.

In general, a network of the type IC WDM PON is more easily scalable in terms of number of users than a DC WDM PON. Changing the size of the WR in the last stage in a DC WDM PON leads to changing the whole network.

\section{Temperature fluctuations}

The influence of temperature fluctuations along the network could be a topic. In reality, the last stages are located far away from the CO. Therefore, the influence of temperature fluctuations has a larger impact in the last stages.

In the case of an IC WDM PON, the coarseness factor is large in the last stage and, therefore, it results in wide passing bands. A small shift of the transfer function implies that only channels on the edge of the passing bands are affected. Channels with a relatively small distance to the center of the passing band can fluctuate without disturbance due to the continuous flat transfer function. 
In a DC WDM PON, the coarseness is small in the last stage and results in narrow passing bands. A small shift of the transfer function due to temperature fluctuations could concern many channels. This means that channels can be easily suppressed or can be easily rerouted to an undesired user.

In summary, both IC and DC WDM PON networks are affected by temperature fluctuations. But networks with IC have a slight benefit compared with networks with a DC property due to the fewer channels affected. This benefit is relative because we did not permit any incorrectly routed channels.

\subsubsection{Summary}

The set of equations described in this section allows the evaluation of all the possible network parameters that describe an IC WDM PON and a DC WDM PON. The set of equations describes the design independent variables $U, U_{\omega}, \vec{M}, \vec{N}$ which are linked with the design dependent variables $\overrightarrow{\mathrm{I}}, \overrightarrow{\mathrm{c}}, \mathrm{n}_{\omega}, \mathrm{N}_{\omega}$.

The design dependent variable I can be calculated with equation (5.15) and (5.20) independent from the type of the considered WDM PON. The coarseness factor can be determined with equation (5.26) for an IC WDM PON and with equation (5.35) for a DC WDM PON. The number of wavelengths $\mathrm{U}_{\omega}$ can be calculated with equation (5.36) independently from the type of the WDM PON. The parameters $n_{\omega}$ and $N_{\omega}$ can be determined with equations (4.26) and (5.14) respectively.

Applying the methods described in this section, we are able to calculate any multistage WDM PON with respect to the defined design conventions. The objective to fulfill the new paradigm in equation (3.1) could be shown. 


\subsection{Connectivity Map}

In a multistage WDM PON, it is essential to understand which source (wavelength) in the OLT is connected to which ONU. The connectivity map can be built by inspection. But in practice, it is very cumbersome for a large amount of used wavelengths, ONUs and stages. Therefore, a planning tool to automatically determine each wavelength in each stage has been developed. This tool is based on a discrete model in the wavelength domain (see section 3.1).

For a large number of wavelengths in the WDM PON, it is important to know what wavelength is received by which ONU. This can be stated inversely to inquire which wavelength must be transmitted by the sources in the OLT and on what input port at the first stage to reach a designated ONU. Especially for network enhancements (in case of wavelengths), we must know which designated wavelengths must be put into which input port in the first stage.

In the presented model, the connectivity map of any WDM PON architecture with a tree topology is described by a wavelength transfer matrix (see chapter 3). The model needs only the input pattern of a discrete optical WDM signal and calculates the mapped output signals (wavelengths).

As mentioned previously in chapters 3 and 4, no wavelength conversion is used and therefore the dimension of the wavelength domain is constant throughout the whole network.

In our model, the PON is partitioned in stages and interstages as shown in Figure 4.5. Generally, there are two possibilities to calculate the entire network. The first one begins at the OLT and goes down stage by stage towards the ONUs (subsection 4.2.1). The second one begins at the ONUs and then goes up towards the OLT stage by stage (subsection 4.2.2). I think it is convenient to begin at the OLT and then go down in the direction of the ONUs.

In Figure 4.5, the modeled architecture to describe the multistage WDM PON is presented. The whole WDM PON is partitioned in stages and interstages. A stage contains optical devices located at the same level of the tree. An interstage consists of optical fibers that interconnect two consecutive stages. Stages are numbered from 0 to $\mathrm{S}$ whereas interstages are numbered from 1 to $\mathrm{S}+1$. Stage 0 represents the OLT, which is located at the central office. The last stage $\mathrm{S}$ connects all ONUs directly via the interstage $\mathrm{S}+1$. Stage 0 represents the signals transmitted by the lasers in the $\mathrm{CO}$ and can be modeled with a matrix. In interstage 1, the dimension of the output matrix $\left(\mathrm{OUT}_{1}\right)$ in stage 0 is $\mathrm{I}_{1} \times \mathrm{n}_{\omega}$ whereas $\mathrm{n}_{\omega}$ is the maximal number of wavelengths per fiber link and $I_{1}$ are the number of fibers in interstage 1 and can be determined with equation (5.15). 


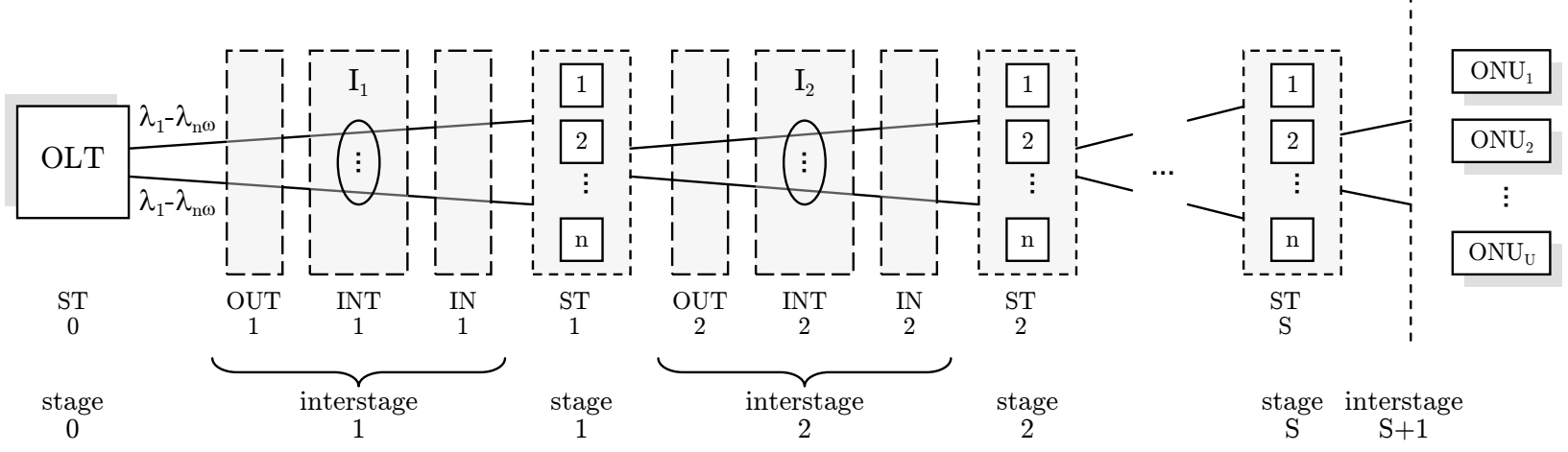

Figure 4.5: Architecture of the multistage WDM PON used by the calculation of the connectivity map.

Each element in all matrices uses integer numbers to represent a particular channel (wavelength) and the adequate fiber number. The notation of the integer number is illustrated in Figure 4.6. It has a minimum size of five digits. The lowest four digits represents the used channel (assuming the highest wavelength with 9999). The digits beginning with the fifth digit represents the used fiber number in the first interstage $\left(\mathrm{INT}_{1}\right)$.

\subsubsection{Top-Down Approach}

The top-down approach begins at the OLT and then goes down stage by stage towards the ONUs. First of all, the interstage was modelled in a matrix representation. In the case of an interstage $\mathrm{s}$, connecting stage $\mathrm{s}-1$ with stage $\mathrm{s}$, we define an interstage operator $\mathrm{INT}_{\mathrm{s}}$ which represents the interstage connectivity function. The dimension of the interstage operator $\mathrm{INT}_{\mathrm{s}}$ must be $\mathrm{I}_{\mathrm{s}} \mathrm{x} \mathrm{I}_{\mathrm{s}-1}$. The input matrix in stage $\mathrm{s}\left(\mathrm{IN}_{\mathrm{s}}\right)$ can be calculated by the following matrix product

$$
\mathrm{IN}_{\mathrm{S}}=\mathrm{INT}_{\mathrm{S}} \cdot \mathrm{OUT}_{\mathrm{s}}
$$

An example is illustrated in Figure 4.6 in which OUT $_{\text {s }}$ is working with 4 inputs (number of rows in $\mathrm{OUT}_{\mathrm{s}}$ ). The wavelength range for all inputs are from 1 to 4 (number of columns in $\mathrm{OUT}_{\mathrm{s}}$ ) and is the same for all inputs. The outcome of the matrix multiplication is the matrix $\mathrm{IN}_{\mathrm{s}}$, in which the spatial assignment can be realized. The dimension of the resulted matrix $\mathrm{IN}_{\mathrm{s}}$ must be $\mathrm{I}_{\mathrm{s}} \times \mathrm{n}_{\omega}$ as demonstrated in Figure 4.6.

For modelling the routing function of a WR, we use a matrix representation similar to the interstage matrix. In the case of stage s connecting interstage $\mathrm{s}$ and $\mathrm{s}+1$, we define a stage operator $\mathrm{ST}_{\mathrm{s}}$. The stage operator $\mathrm{ST}_{\mathrm{s}}$ represents the space switching function of the optical devices in stage s. The output matrix $\mathrm{OUT}_{\mathrm{s}}$ can be calculated by the product of the input matrix $\mathrm{IN}_{\mathrm{s}}$ and an operator $\mathrm{ST}_{\mathrm{s}}$ representing the $\mathrm{s}^{\text {th }}$ stage connectivity function

$$
\mathrm{OUT}_{\mathrm{S}+1}=\mathrm{ST}_{\mathrm{S}} \cdot \mathrm{IN}_{\mathrm{S}} \quad \mathrm{s} \in[1, \mathrm{~S}]
$$




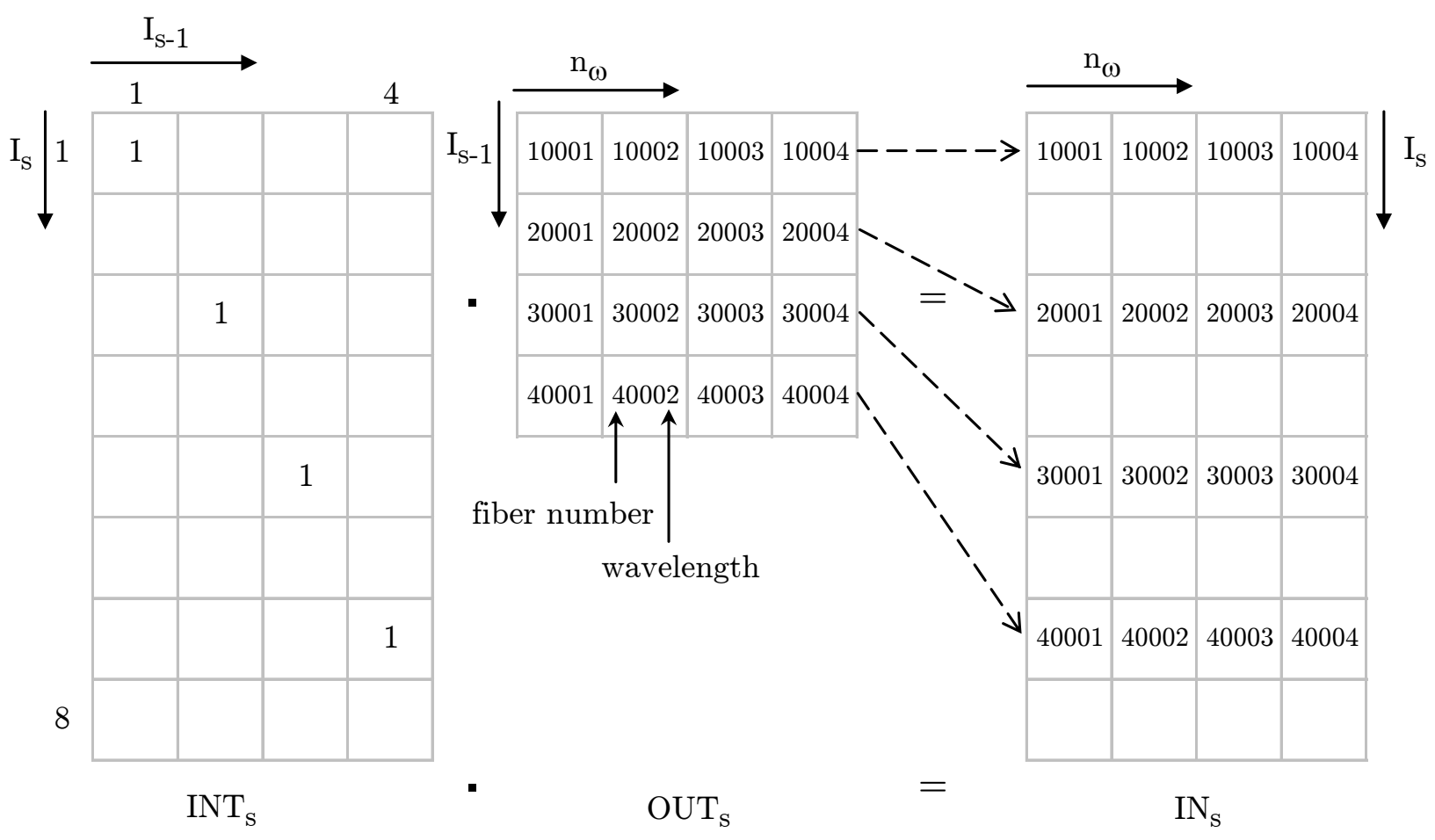

Figure 4.6: Two-dimensional interstage operator with its input and output matrices with $\mathrm{n}_{\omega}=4, \mathrm{I}_{\mathrm{s}-1}=4$ and $I_{s}=8$.

The stage operator must express the correct space switching function that depends on the number of wavelengths and the used coarseness factor in stage s. For that reason, the stage operator looks like a three-dimensional matrix (Figure 4.7). In mathematics, there is no multiplication defined for three-dimensional matrices. Therefore, we define the multiplication as follows: the $\mathrm{n}^{\text {th }}$ bidimensional matrix of the stage operator multiplies the $\mathrm{n}^{\text {th }}$ column of the input matrix $\mathrm{IN}_{\mathrm{s}}$. Other columns are not affected by the multiplication. The result is a column vector in the output matrix $\mathrm{OUT}_{\mathrm{s}+1}$ (see Figure 4.7). Due to the routing function performed by the optical devices in stage $\mathrm{s}$, the labels in the matrix $\mathrm{OUT}_{\mathrm{s}+1}$ are shifted. This multiplication must be done for all $\mathrm{n}_{\omega}$ wavelengths per link.

The dimension of the input signal matrix in stage $\mathrm{s}\left(\mathrm{IN}_{\mathrm{s}}\right)$ is $\mathrm{I}_{\mathrm{s}} \times \mathrm{n}_{\omega}$, of the stage operator $\left(\mathrm{ST}_{\mathrm{s}}\right) \mathrm{I}_{\mathrm{s}} \times \mathrm{I}_{\mathrm{s}} \times \mathrm{n}_{\omega}$ and the dimension of the produced output matrix $\left(\mathrm{OUT}_{\mathrm{s}}\right)$ is $\mathrm{I}_{\mathrm{s}} \times \mathrm{n}_{\omega}$. The routing function for the first wavelength (first column of the $\mathrm{IN}_{\mathrm{s}}$ matrix) are routed to the same output port number as the input port and can be expressed by the identity matrix. The routing property for the next wavelength can be described by the identity matrix which is moved one position in the $\mathrm{y}$ direction. The shifting of the identity matrix is expressed by an operator called Move $_{n}$ (see Figure 4.7). The subscripted index $\mathrm{n}$ describes the number of digits, the identity matrix is moved in the $\mathrm{y}$-direction.

As mentioned before and shown in Figure 4.7, the stage operator is a three-dimensional matrix. It is important to understand that the structure of the three-dimensional stage operator depends on two factors: the number of used wavelengths and the coarseness factor. 


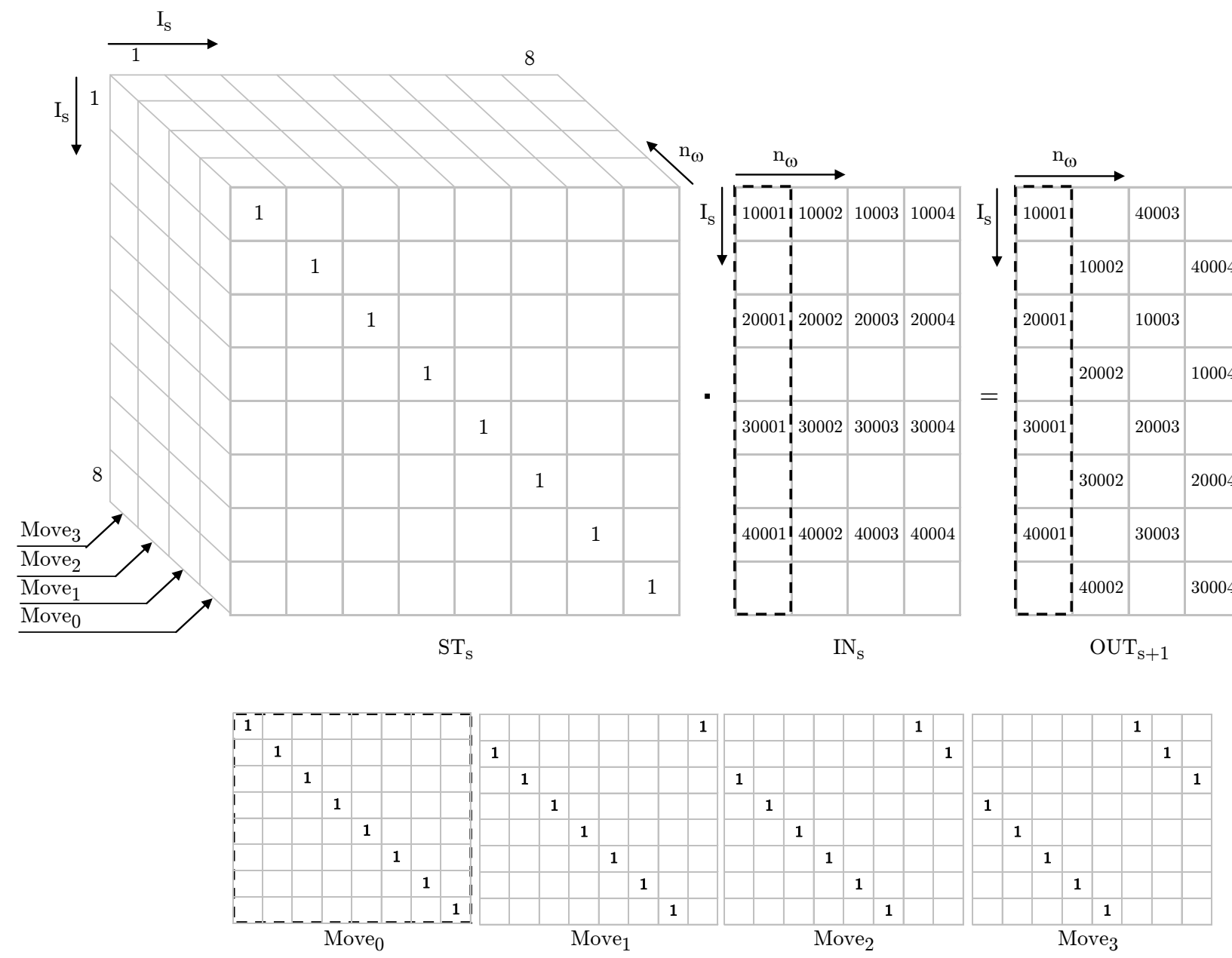

Figure 4.7: Three-dimensional stage operator with its input and output matrices for $\mathrm{n}_{\omega}=4, \mathrm{c}=1$ and $\mathrm{M}=8$.

Let us assume a simple example with a coarseness factor of $\mathrm{c}=1$ and a number of wavelengths of $\mathrm{n}_{\omega} \leq \mathrm{M}$. The structure of the stage operator can be determined by looking at equation (4.3). The position of the output port of a certain wavelength depends on the position of the input port, the coarseness factor and the size of the WR. A given wavelength $\mathrm{k}$ is circular shifted by $\mathrm{k}-1$ positions.

As a consequence, the three-dimensional stage operator consists of $\mathrm{n}_{\omega}$ bidimensional $\mathrm{M} \mathrm{x}$ $\mathrm{M}$ matrices. Each of the $\mathrm{n}_{\omega}$ bidimensional matrices must perform a circular shift of the corresponding column in the input matrix IN. As a consequence, a Move $\mathrm{k}_{\mathrm{k}}$ operator which is able to accomplish an order $\mathrm{k}$ shift must be defined. The wavelength $\mathrm{k}$ corresponds to the matrix Move $_{\mathrm{k}-1}$. The Move matrix which corresponds to the wavelength $\mathrm{k}=1$ is $\mathrm{Move}_{0}$ and represents the identity matrix. Figure 4.7 exhibits the complete three-dimensional stage operator and its corresponding Move matrices for an 8 $\mathrm{x} 8 \mathrm{WR}$ with $\mathrm{n}_{\omega}=4$ and $\mathrm{c}=1$.

If the coarseness factor $\mathrm{c}$ is greater than one, the three-dimensional stage operator can be built by copying the Move matrices c times. This procedure is independent from the number of used wavelengths. In Figure 4.8 the overall $3-\mathrm{D}$ stage operator associated with an $8 \times 8 \mathrm{WR}$ for $\mathrm{n}_{\omega}=4$ and $\mathrm{c}=2$ is demonstrated. 


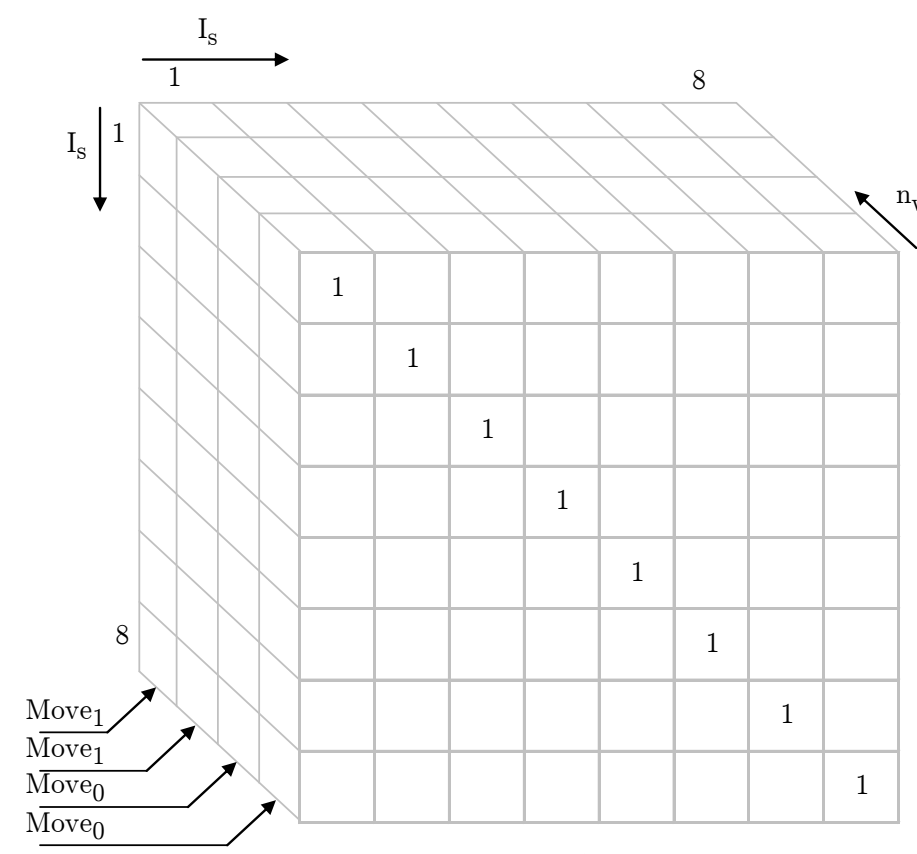

$\mathrm{ST}_{\mathrm{S}}$

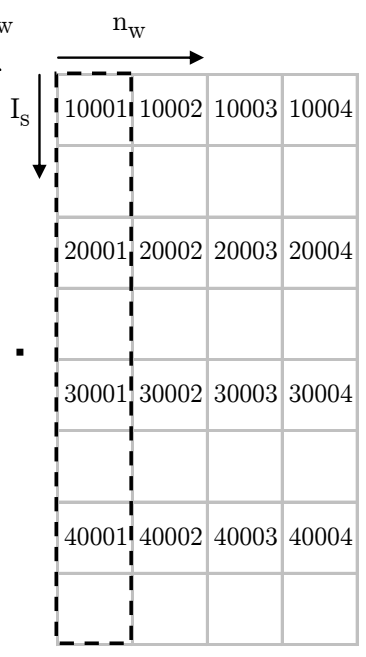

$\mathrm{IN}_{\mathrm{S}}$

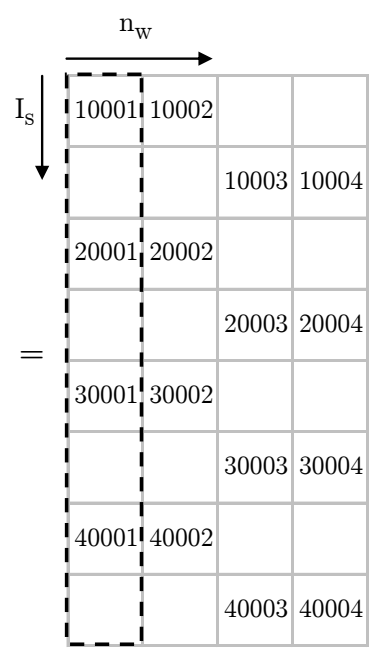

$\mathrm{OUT}_{\mathrm{S}+1}$

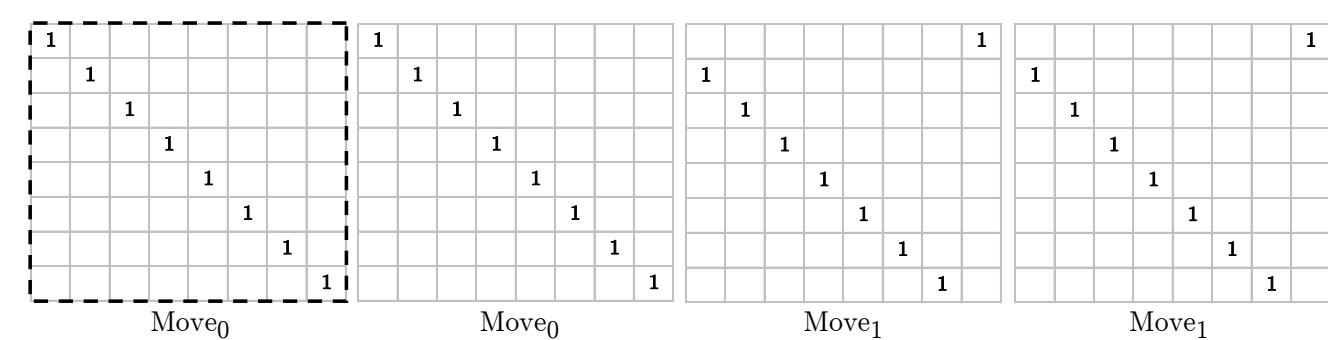

Figure 4.8: Three-dimensional stage operator with its input and output matrices for $\mathrm{n}_{\omega}=4, \mathrm{c}=2$ and $\mathrm{M}=8$. The Move matrices are copied two times by reason of $\mathrm{c}=2$.

In the case in which the number of wavelengths $n_{\omega}$ is greater than the size $M$ of the WR, the wavelengths exceeding $M$ must be cyclically routed, according to the cyclic routing property of the WR (see equation (4.3)). From there, the stage operator must be adapted. The first set of $\mathrm{M}$ bidimensional Move matrices are identically repeated along the $\mathrm{n}_{\omega}$-axis of the stage operator $\mathrm{ST}_{\mathrm{s}}$. The index of the Move operator for wavelength $\mathrm{k}$ can be obtained by

$$
\operatorname{Move}_{\left(\left\lfloor\frac{\mathrm{k}}{\mathrm{c}}\right\rfloor-1\right) \bmod \mathrm{M}}
$$

The index of the Move operator contains the term $\left\lfloor\frac{\mathrm{k}}{\mathrm{c}}\right\rfloor-1$ which take into account the coarseness factor and corresponds to wavelength $\mathrm{k}$. The operator modulo is accounted for the cyclic routing property of the WR.

In Figure 4.9, the entire 3-D stage operator and its corresponding Move matrices for a WR with $\mathrm{M}=4, \mathrm{n}_{\omega}=6$ and $\mathrm{c}=1$ is demonstrated. 


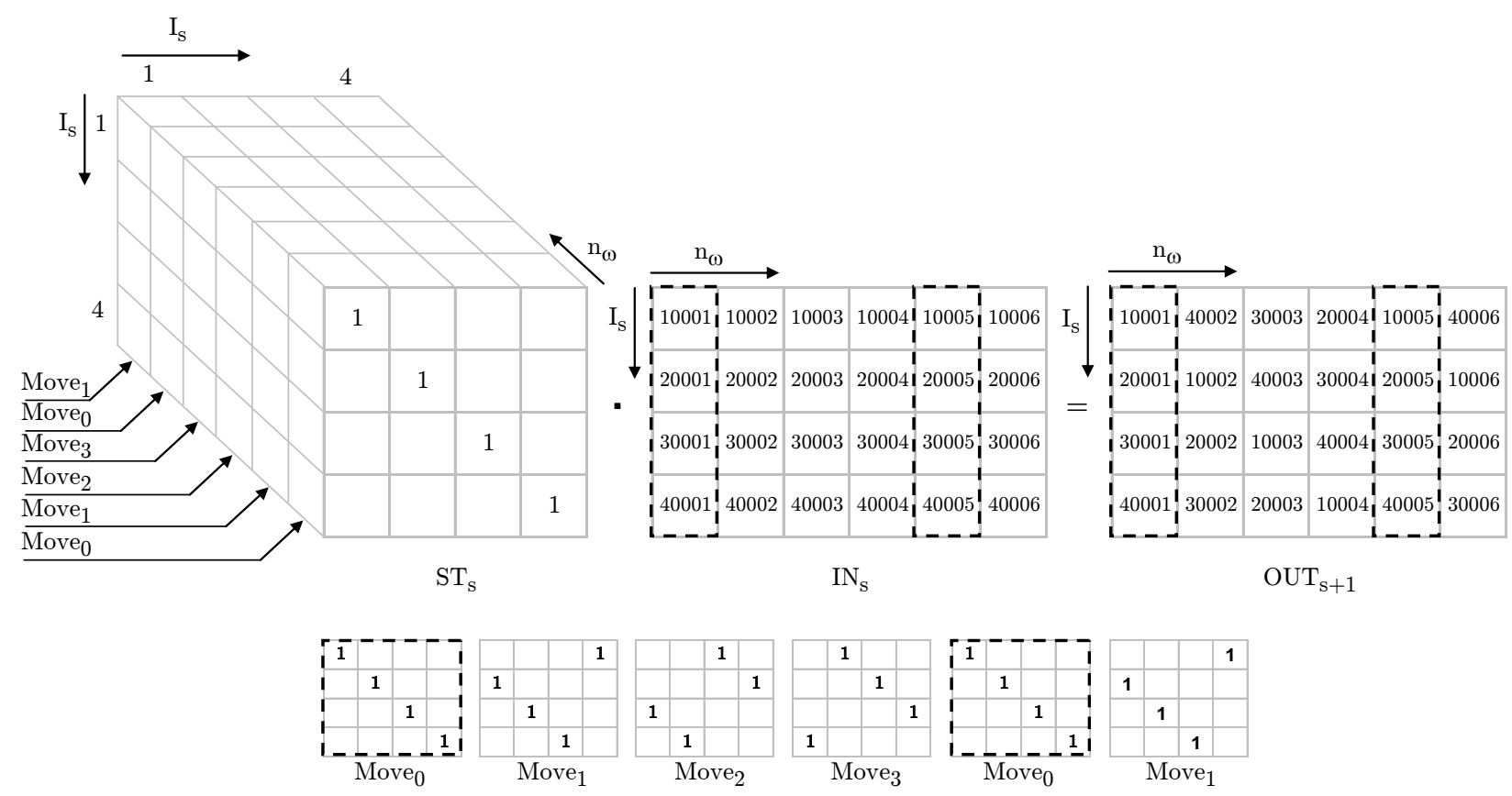

Figure 4.9: Three-dimensional stage operator with its input and output matrices for $\mathrm{n}_{\omega}=6, \mathrm{c}=1$ and $\mathrm{M}=4$. The number of wavelengths $n_{\omega}$ is greater than the dimension $M$ of the WR. As a consequence, the Move matrices are identically repeated along the $\mathrm{n}_{\omega}$-axis of the stage operator.

With both equations (5.37) and (5.38), the routing function of any stage or interstage in the network tree can be calculated. The signals transmitted by the sources in the CO $\left(\mathrm{OUT}_{1}\right)$ are routed through all stages and interstages to the ONUs. The signal flow through the whole WDM PON can be determined by the connection of the interstage and stage operators in series. Expressed by matrix multiplications leads to

$$
\begin{gathered}
\mathrm{IN}_{1}=\mathrm{INT}_{1} \cdot \mathrm{OUT}_{1} \\
\mathrm{OUT}_{2}=\mathrm{ST}_{1} \cdot \mathrm{IN}_{1}=\mathrm{ST}_{1} \cdot \mathrm{INT}_{1} \cdot \mathrm{OUT}_{1} \\
\mathrm{IN}_{2}=\mathrm{INT}_{2} \cdot \mathrm{OUT}_{2}=\mathrm{INT}_{2} \cdot \mathrm{ST}_{1} \cdot \mathrm{INT}_{1} \cdot \mathrm{OUT}_{1} \\
\mathrm{OUT}_{3}=\mathrm{ST}_{2} \cdot \mathrm{IN}_{2}=\mathrm{ST}_{2} \cdot \mathrm{INT}_{2} \cdot \mathrm{ST}_{1} \cdot \mathrm{INT}_{1} \cdot \mathrm{OUT}_{1} \\
\mathrm{IN}_{3}=\mathrm{INT}_{3} \cdot \mathrm{OUT}_{3}=\mathrm{INT}_{3} \cdot \mathrm{ST}_{2} \cdot \mathrm{INT}_{2} \cdot \mathrm{ST}_{1} \cdot \mathrm{INT}_{1} \cdot \mathrm{OUT}_{1} \\
\ldots \\
\mathrm{IN}_{\mathrm{ONU}}=\mathrm{INT}_{\mathrm{S}+1} \cdot \mathrm{OUT}_{\mathrm{S}+1}=\mathrm{INT}_{\mathrm{S}+1} \cdot \mathrm{ST}_{\mathrm{S}} \cdot \mathrm{INT}_{\mathrm{S}} \cdot \ldots \cdot \mathrm{INT}_{1} \cdot \mathrm{OUT}_{1} \cdot
\end{gathered}
$$

The resulted matrix $\mathrm{IN}_{\mathrm{ONU}}$ describes all signals reaching the ONUs and can be considered as the output (OUT) of the whole WDM PON. Rewriting equation (5.45) in a compact form results in

$$
\mathrm{OUT}=\mathrm{NET} \cdot \mathrm{OUT}_{1}
$$

The NET operator represents the discrete transfer function of the whole WDM PON. The NET operator is the matrix product of all stage and interstage operators and can be expressed as

$$
\mathrm{NET}=\mathrm{INT}_{\mathrm{S}+1} \cdot \mathrm{ST}_{\mathrm{S}} \cdot \mathrm{INT}_{\mathrm{S}} \cdot \mathrm{ST}_{\mathrm{S}-1} \cdot \mathrm{INT}_{\mathrm{S}-1} \cdot \mathrm{ST}_{\mathrm{S}-2} \cdot \ldots \cdot \mathrm{ST}_{1} \cdot \mathrm{INT}_{1}
$$


The interstage operator can also be written in a compact notation with the product symbol

$$
\mathrm{NET}=\mathrm{INT}_{\mathrm{S}+1} \prod_{\mathrm{x}=1}^{\mathrm{S}} \mathrm{ST}_{\mathrm{x}} \cdot \mathrm{INT}_{\mathrm{x}}
$$

The NET operator is automatically acquired in the connectivity mapping tool. It requires the information obtained from the previous network design process described in section 4.1 which includes the parameters $\overrightarrow{\mathrm{M}}, \overrightarrow{\mathrm{I}}, \overrightarrow{\mathrm{N}}, \overrightarrow{\mathrm{c}}, \mathrm{n}_{\omega}$ for all stages. In the connectivity mapping tool, all stage and interstage operations are traced back to normal $2 \mathrm{D}$ matrix multiplications.

The whole WDM PON consists of $\mathrm{S}$ stages and $\mathrm{S}+1$ interstages. Therefore, the number of $3 \mathrm{D}$ matrix multiplications is $2 \mathrm{~S}+1$. Each interstage requires only one $2 \mathrm{D}$ matrix multiplication whereas each stage requires $\mathrm{n}_{\omega} 2 \mathrm{D}$ matrix multiplications. As a result, the calculation of the connectivity map of the whole WDM PON needs

$$
\mathrm{S}+1+\mathrm{Sn}_{\omega}
$$

matrix multiplications. For a large number of $\mathrm{n}_{\omega}$, the required matrix multiplications can be aproximately calculated by $\mathrm{Sn}_{\omega}$ matrix multiplications.

Due to limitations in the power budget (see section 4.4), a maximum of five stages are technologically feasible. Thus, the number of matrix multiplications to calculate the whole WDM PON is $6+5 \mathrm{n}_{\omega}$. In summary, the used method results in a low complexity. Additionally, the required memory to save the stage and interstage matrices is minimized due to the resulted sparse matrix.

For many questions it is important to calculate the connectivity map in the opposite direction. The method of resolution is illustrated and discussed in detail in the next subsection. 


\subsubsection{Bottom-Up Approach}

The bottom-up approach begins at the ONUs and then goes up stage by stage towards the OLT. As a consequence, the stage and interstage operators from the top-down approach in subsection 4.2.1 cannot be used. Therefore, new stage and interstage operators alike must be defined by using the model in Figure 4.5, as in subsection 4.2.1. Similar to the interstage operator in equation (5.37), an inverse interstage operator $\mathrm{INT}_{\mathrm{s}}^{-1}$ for stage $\mathrm{s}$ must be defined. The inverse interstage operator represents the connectivity between stage $\mathrm{s}$ and stage $\mathrm{s}-1$. The output matrix $\mathrm{OUT}_{\mathrm{s}}$ can be expressed by the following matrix product

$$
\mathrm{OUT}_{\mathrm{S}}=\mathrm{INT}_{\mathrm{S}}^{-1} \cdot \mathrm{IN}_{\mathrm{S}} \quad \mathrm{s} \in[1, \mathrm{~S}+1]
$$

From the interstage operator (5.37) we know that the dimension of the matrix $\mathrm{OUT}_{\mathrm{s}}$ is $I_{s-1} \times n_{\omega}$, and the matrix $I N_{s}$ is $I_{s} x_{\omega}$. For that reason, the inverse interstage operator $\mathrm{INT}_{\mathrm{s}}^{-1}$ must have a dimension of $\mathrm{I}_{\mathrm{s}-1} \mathrm{x} \mathrm{I}_{\mathrm{s}}$. An example of the inverse interstage operator is given in Figure 4.10 for $\mathrm{n}_{\omega}=4, \mathrm{I}_{\mathrm{s}}=8$ and $\mathrm{I}_{\mathrm{s}-1}=4$.
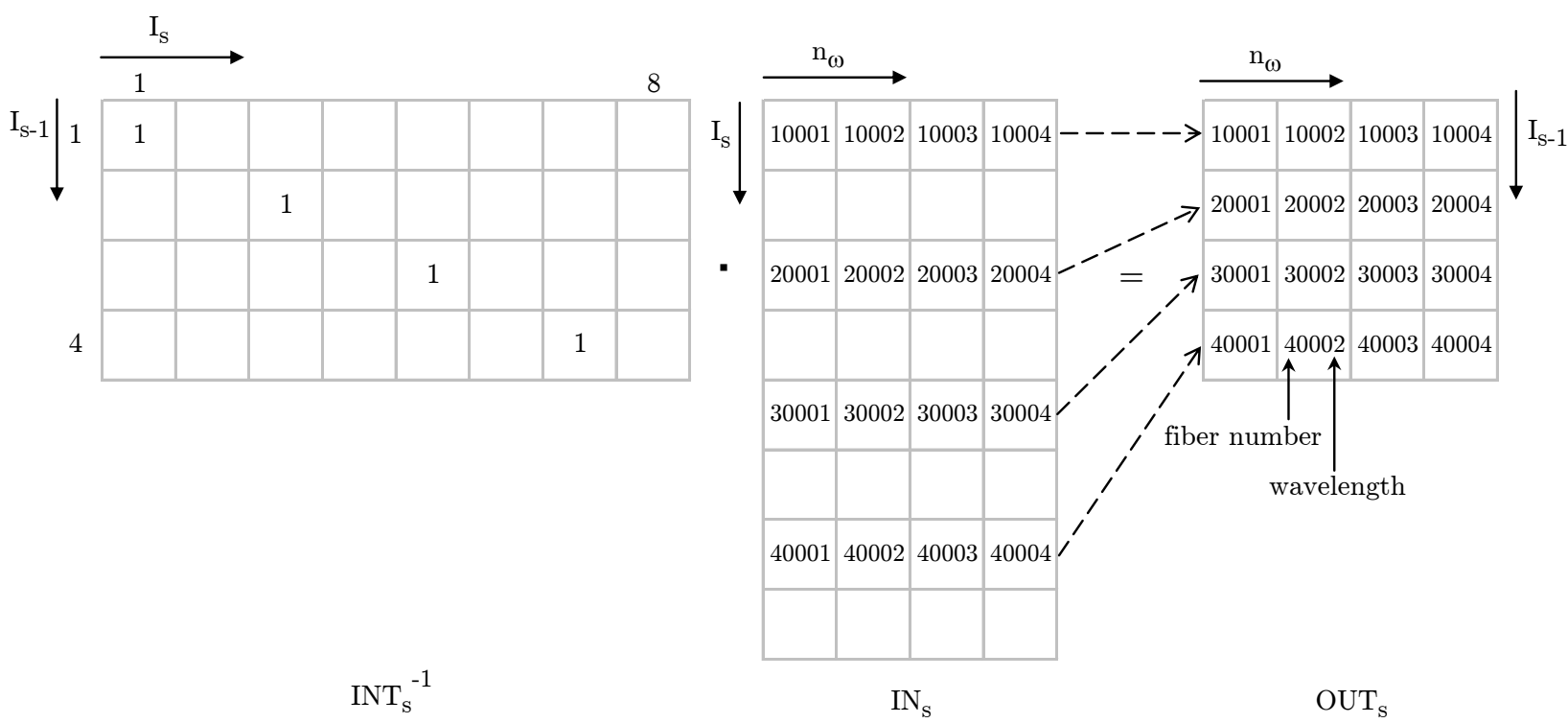

Figure 4.10: Two-dimensional inverse interstage operator with its input and output matrices for $\mathrm{n}_{\omega}=4$, $\mathrm{I}_{\mathrm{s}}=8$ and $\mathrm{I}_{\mathrm{s}-1}=4$.

After the matrix multiplication, the spatial assignment can be realized in $\mathrm{OUT}_{\mathrm{s}}$.

In the next step an inverse stage operator that represents the inverse space switching function must be defined. The inverse stage operator assigns the wavelengths in stage $\mathrm{s}+1$ to stage $\mathrm{s}$. The formal expression is given by

$$
\mathrm{IN}_{\mathrm{S}}=\mathrm{ST}_{\mathrm{S}}^{-1} \cdot \mathrm{OUT}_{\mathrm{S}+1} \quad \mathrm{~s} \in[1, \mathrm{~S}]
$$

Even with the stage operator, the inverse stage operator depends on the number of wavelengths used and the coarseness factor. Therefore, the inverse stage operator is similar to the stage operator as a three-dimensional matrix (Figure 4.11). The main 
difference is the Move Operator. Compared with the stage operator, the unitary matrix must be shifted in the negative y-direction as shown in Figure 4.11. This characteristic is marked with a negative sign in the index of the Move Operator.

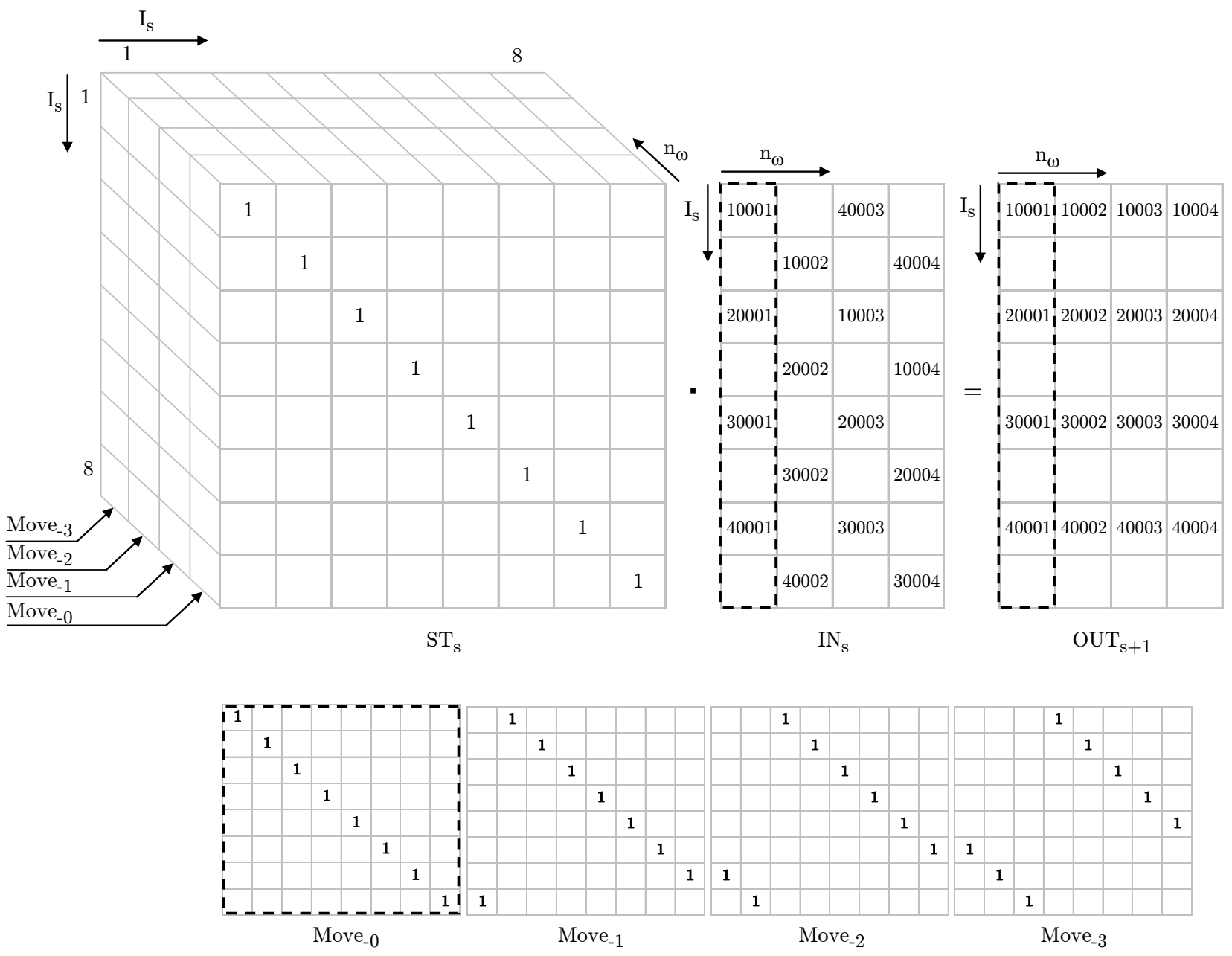

Figure 4.11: Inverse 3D stage operator with its input and output matrices with $\mathrm{n}_{\omega}=4, \mathrm{c}=1$ and $\mathrm{M}=8$.

As in the top-down approach, in the case in which the number of wavelengths $\mathrm{n}_{\omega}$ is greater than the size $M$ of the WR, the wavelengths exceeding $M$ must be cyclically routed according to the cyclic routing property of the WR. The difference is the value of the movement of the unity matrix. The first set of $\mathrm{M}$ bidimensional Move matrices are identically repeated along the $\mathrm{n}_{\omega}$-axis of the stage operator $\mathrm{ST}_{\mathrm{s}}$. The index of the Move operator for wavelength $\mathrm{k}$ can be obtained by

$$
{ }^{\text {Move }}-\left(\left\lfloor\frac{\mathrm{k}}{\mathrm{c}}\right\rfloor-1\right) \bmod \mathrm{M}
$$

With the equations (5.50) and (5.51), we are able to describe the signal flow through the whole WDM PON, starting from the ONUs and ending at the OLT by using the inverse stage and inverse interstage operator. The signals received from the ONUs in interstage $\mathrm{S}+1\left(\mathrm{IN}_{\mathrm{S}+1}\right)$ are routed through all interstages and stages. The signal flowing through all stages and interstages can be described by the following relationship 


$$
\begin{gathered}
\mathrm{OUT}_{\mathrm{S}+1}=\mathrm{INT}_{\mathrm{S}+1}^{-1} \cdot \mathrm{IN}_{\mathrm{S}+1} \\
\mathrm{IN}_{\mathrm{S}}=\mathrm{ST}_{\mathrm{S}}^{-1} \cdot \mathrm{OUT}_{\mathrm{S}+1}=\mathrm{ST}_{\mathrm{S}}^{-1} \cdot \mathrm{INT}_{\mathrm{S}+1}^{-1} \cdot \mathrm{IN}_{\mathrm{S}+1} \\
\mathrm{OUT}_{\mathrm{S}}=\mathrm{INT}_{\mathrm{S}}^{-1} \cdot \mathrm{IN}_{\mathrm{S}}=\mathrm{INT}_{\mathrm{S}}^{-1} \cdot \mathrm{ST}_{\mathrm{S}}^{-1} \cdot \mathrm{INT}_{\mathrm{S}+1}^{-1} \cdot \mathrm{IN}_{\mathrm{S}+1} \\
\mathrm{IN}_{\mathrm{S}-1}=\mathrm{ST}_{\mathrm{S}-1}^{-1} \cdot \mathrm{OUT}_{\mathrm{S}}=\mathrm{ST}_{\mathrm{S}-1}^{-1} \cdot \mathrm{INT}_{\mathrm{S}}^{-1} \cdot \mathrm{ST}_{\mathrm{S}}^{-1} \cdot \mathrm{INT}_{\mathrm{S}+1}^{-1} \cdot \mathrm{IN}_{\mathrm{S}+1} \\
\mathrm{OUT}_{\mathrm{S}-1}=\mathrm{INT}_{\mathrm{S}-1}^{-1} \cdot \mathrm{IN}_{\mathrm{S}-1}=\mathrm{INT}_{\mathrm{S}-1}^{-1} \cdot \mathrm{ST}_{\mathrm{S}-1}^{-1} \cdot \mathrm{INT}_{\mathrm{S}}^{-1} \cdot \mathrm{ST}_{\mathrm{S}}^{-1} \cdot \mathrm{INT}_{\mathrm{S}+1}^{-1} \cdot \mathrm{IN}_{\mathrm{S}+1} \\
\ldots \\
\mathrm{OUT}_{1}=\mathrm{INT}_{1}^{-1} \cdot \mathrm{IN}_{1}=\mathrm{INT}_{1}^{-1} \cdot \mathrm{ST}_{1}^{-1} \cdot \mathrm{INT}_{2}^{-1} \cdot \mathrm{ST}_{2}^{-1} \cdot \ldots \cdot \mathrm{INT}_{\mathrm{S}+1}^{-1} \cdot \mathrm{IN}_{\mathrm{S}+1} \cdot
\end{gathered}
$$

The resulted matrix $\mathrm{OUT}_{1}$ represents the transmitted signals from the OLT and can be considered as the input (IN) into the whole WDM PON. Thus, we can aggregate equation (5.58) as follows

$$
\mathrm{IN}=\mathrm{NET}^{-1} \cdot \mathrm{IN}_{\mathrm{S}+1}
$$

The $\mathrm{NET}^{-1}$ operator describes the inverse discrete transfer function of the multistage WDM PON architecture and can be expressed as the product of all inverse stage and all inverse interstage operators according to

$$
\mathrm{NET}^{-1}=\mathrm{INT}_{1}^{-1} \cdot \mathrm{ST}_{1}^{-1} \cdot \mathrm{INT}_{2}^{-1} \cdot \mathrm{ST}_{2}^{-1} \cdot \ldots \cdot \mathrm{ST}_{\mathrm{S}}^{-1} \cdot \mathrm{INT}_{\mathrm{S}+1}^{-1}
$$

or with the product symbol in a compressed notation

$$
\mathrm{NET}^{-1}=\mathrm{INT}_{1}^{-1} \prod_{\mathrm{x}=1}^{\mathrm{S}} \mathrm{ST}_{\mathrm{x}}^{-1} \cdot \mathrm{INT}_{\mathrm{x}+1}^{-1}
$$

With the equations (5.60) and (5.61), we are in the desirable position to be able to calculate the signal flow through the whole multistage WDM PON beginning at the ONUs and then moving up towards to the OLT.

The NET operator is automatically acquired in the connectivity mapping tool. It requires the information from the network design process described in section 4.1. Thus, it appears that the NET operator requires the parameters $\vec{M}, \vec{I}, \vec{N}, \vec{c}, n_{\omega}$ for all stages. All stage and interstage operations are traced back to normal 2D matrix multiplications.

Due to the same number of stages and interstages as in the top-down approach, the number of matrix multiplication is identical with $\mathrm{S}+1+\mathrm{Sn}_{\omega}$.

The main difference of both approaches is the input requirement concerning the wavelengths. In the top-down approach, the set of wavelengths transmitted at the CO can easily be specified. But in the bottom-up approach, the set of wavelengths for each user must be predetermined. As a result of the space multiplexing function, this is not an easy task and can be seen as a drawback of the bottom-up approach. 


\subsubsection{Examples}

In this subsection the correct functionality of the connectivity mapping tool will be demonstrated with two examples. The first one is based on two stages and the second one uses three stages. With respect to the paradigm shift in section 2.5 , we assume 4 wavelengths for each user for both examples. The maximum number of used wavelengths is limited to $16\left(\mathrm{n}_{\omega}=16\right.$, according to [ITU-T G.694.2]). In Figure 4.12, the two-stage architecture with $\mathrm{U}=16, \mathrm{U}_{\omega}=4, \mathrm{n}_{\omega}=16, \overrightarrow{\mathrm{N}}=[1,2], \overrightarrow{\mathrm{M}}=[8,8]$ and $\overrightarrow{\mathrm{I}}=[4,4]$ is shown. The connectivity of the architecture can be seen in Figure 4.14 through Figure 4.15. In order to show the effects, the connectivity map is calculated with different coarseness vectors.
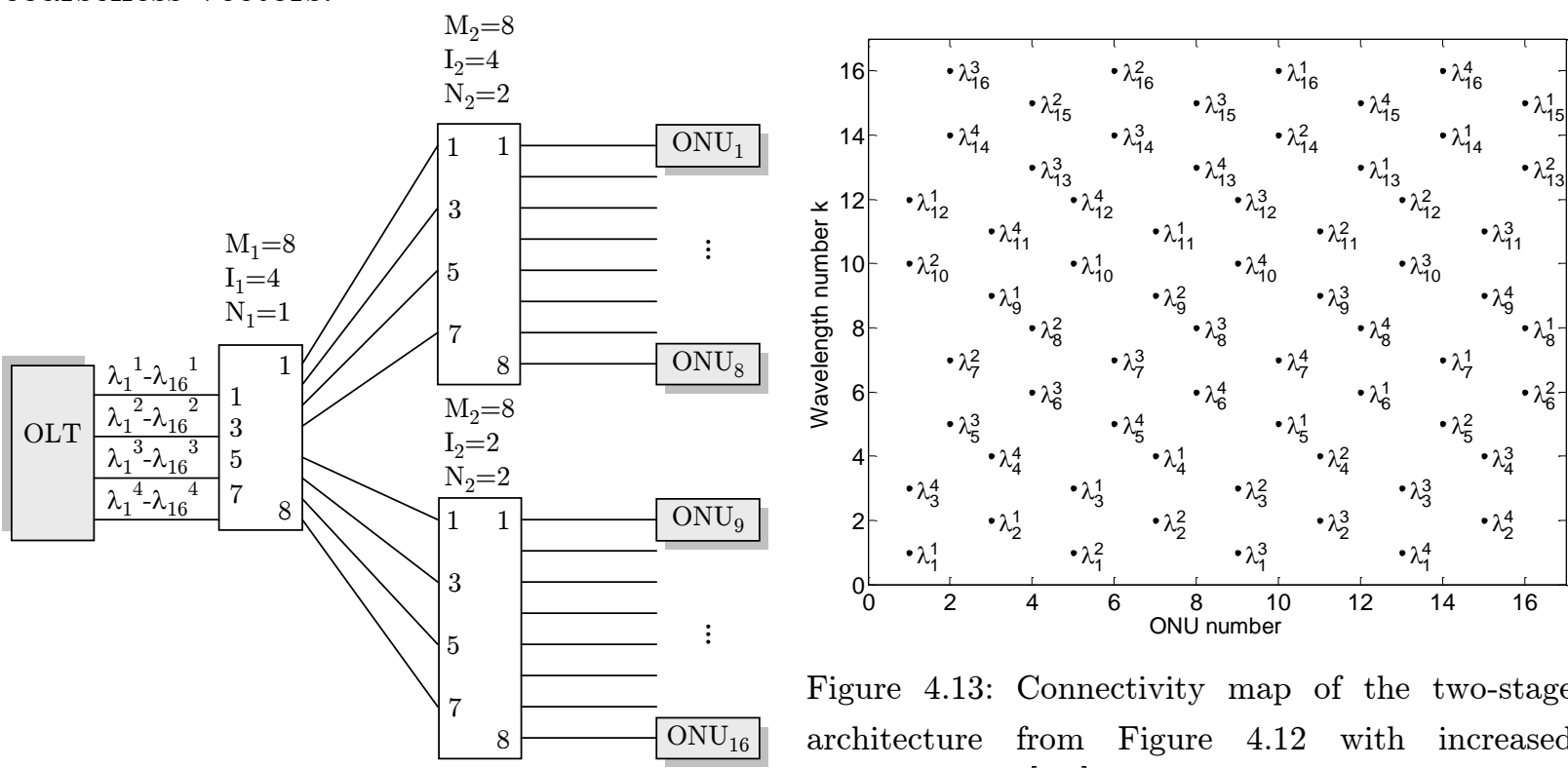

Figure 4.13: Connectivity map of the two-stage architecture from Figure 4.12 with increased coarseness $\overrightarrow{\mathrm{c}}=[1,4]$.

Figure 4.12: Example of a two-stage WDM PON with $\mathrm{U}=16, \mathrm{U}_{\omega}=4, \mathrm{n}_{\omega}=16, \overrightarrow{\mathrm{N}}=[1,2], \overrightarrow{\mathrm{M}}=[8,8]$ and $\overrightarrow{\mathrm{I}}=[4,4]$.

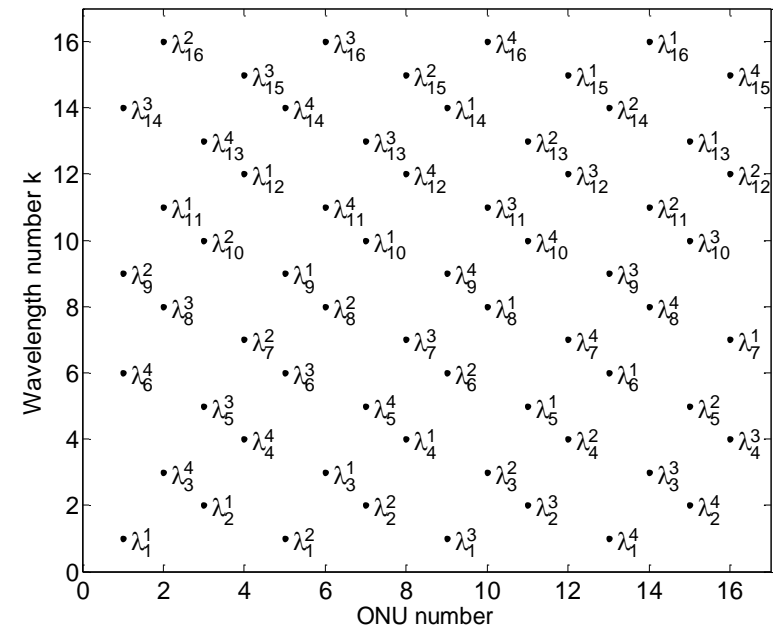

Figure 4.14: Connectivity map of the two-stage architecture from Figure 4.12 with increased coarseness $\overrightarrow{\mathrm{c}}=[1,2]$.

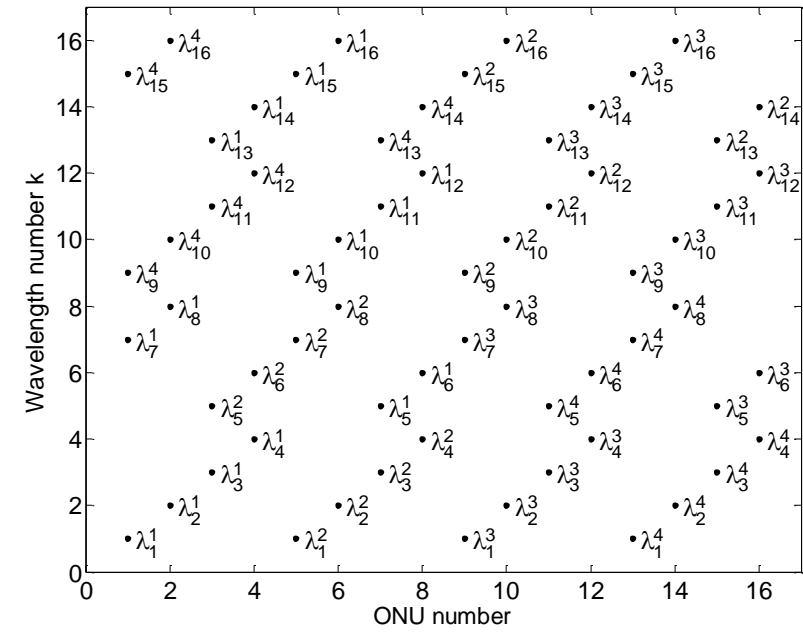

Figure 4.15: Connectivity map of the two-stage architecture from Figure 4.12 with decreased coarseness $\overrightarrow{\mathrm{c}}=[4,1]$. 
Figure 4.14 and Figure 4.13 represent the connectivity map for an IC WDM PON with the coarseness vectors $\overrightarrow{\mathrm{c}}=[1,2]$ and $\overrightarrow{\mathrm{c}}=[1,4]$. Various connectivity patterns can be identified due to the different coarseness vectors. In Figure 4.15, the connectivity map for a DC WDM PON with a coarseness vector of $\overrightarrow{\mathrm{c}}=[4,1]$ is shown. It can be seen that each ONU receives 4 wavelengths and that at the same time wavelength reuse is implemented.

The second example in shows us a three stage architecture with $\mathrm{U}=128, \mathrm{U}_{\omega}=2, \mathrm{n}_{\omega}=16$, $\overrightarrow{\mathrm{N}}=[1,2,2], \overrightarrow{\mathrm{M}}=[32,32,64]$ and $\overrightarrow{\mathrm{I}}=[16,16,32]$. The goal is to provide 2 wavelengths for each user.

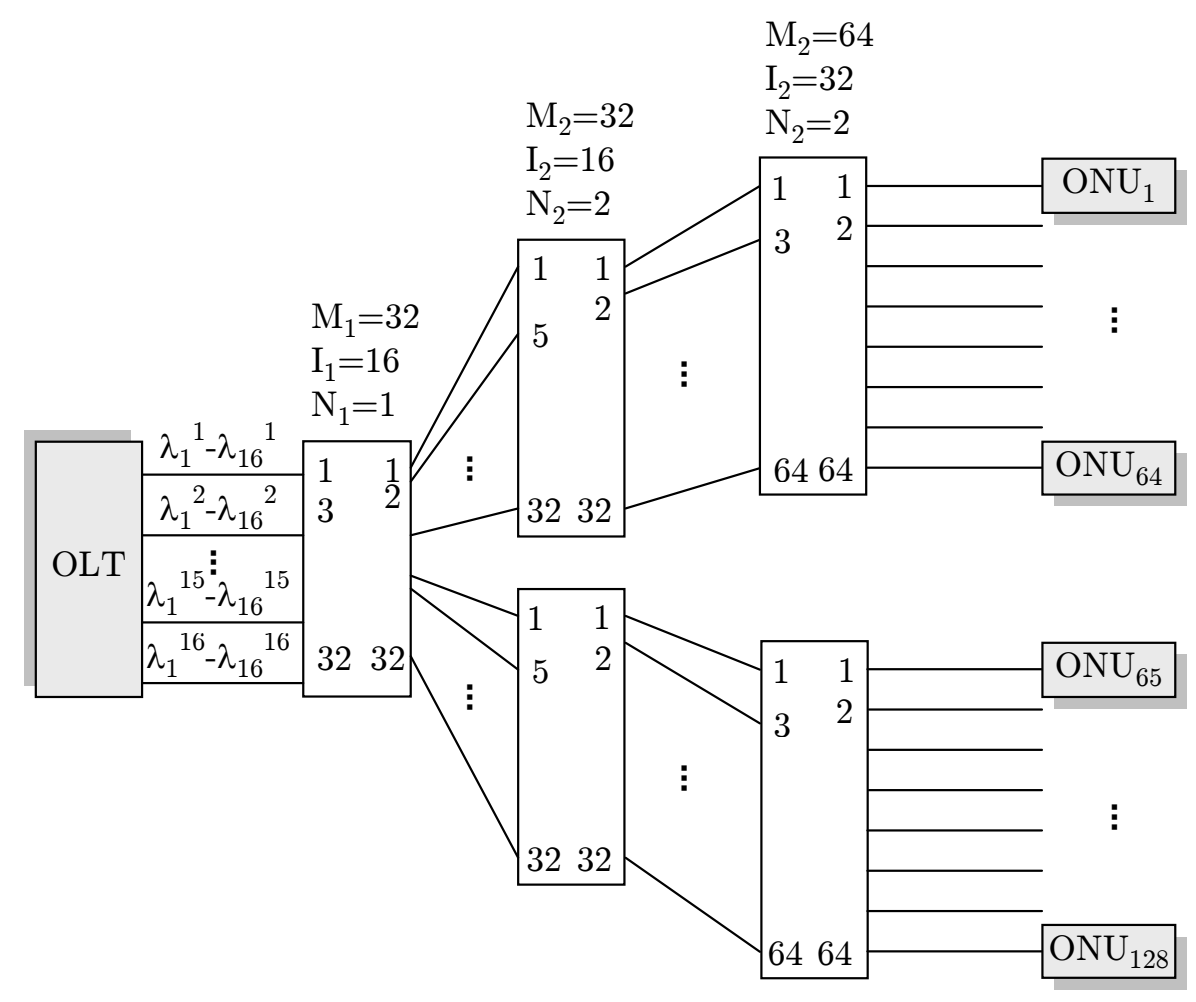

Figure 4.16: Example of a three-stage WDM PON with $\mathrm{U}=128, \mathrm{U}_{\omega}=2, \mathrm{n}_{\omega}=16, \overrightarrow{\mathrm{N}}=[1,2,2]$, $\overrightarrow{\mathrm{M}}=[32,32,64]$ and $\overrightarrow{\mathrm{I}}=[16,16,32]$.

The connectivity pattern for the case $\overrightarrow{\mathrm{c}}=[1,2,4]$ and $\overrightarrow{\mathrm{c}}=[2,4,8]$ is shown in Figure 4.17 and Figure 4.18 respectively. We happily recognize in both cases that each ONU receives exactly two particular wavelengths. A high wavelength reuse can also be seen in both cases.

Through both examples for a two- and three-stage WDM PON, we can demonstrate the same functionality with different coarseness vectors and show how our tool works. 


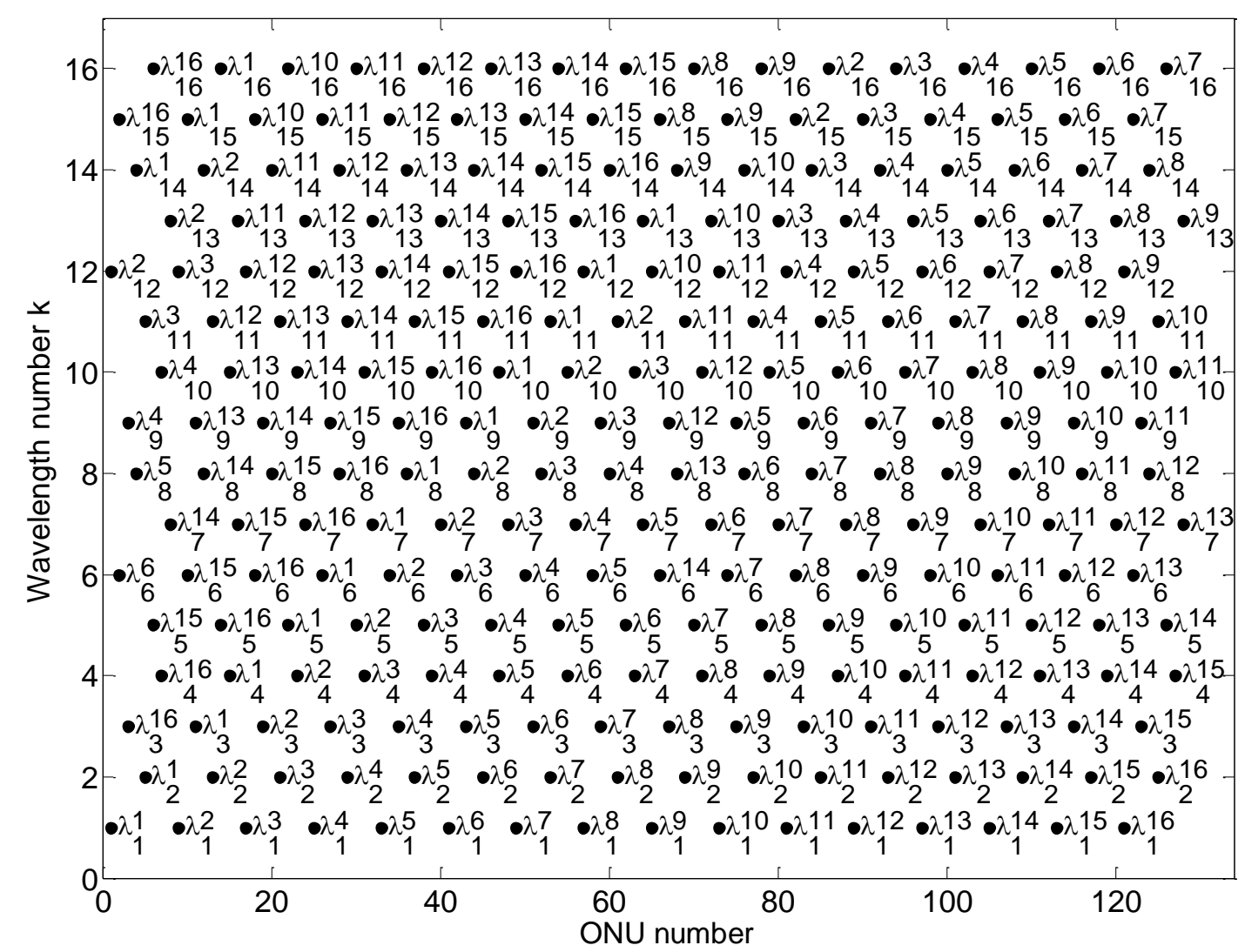

Figure 4.17: Connectivity map of the three-stage architecture from Figure 4.16 with increased coarseness $\overrightarrow{\mathrm{c}}=[1,2,4]$.

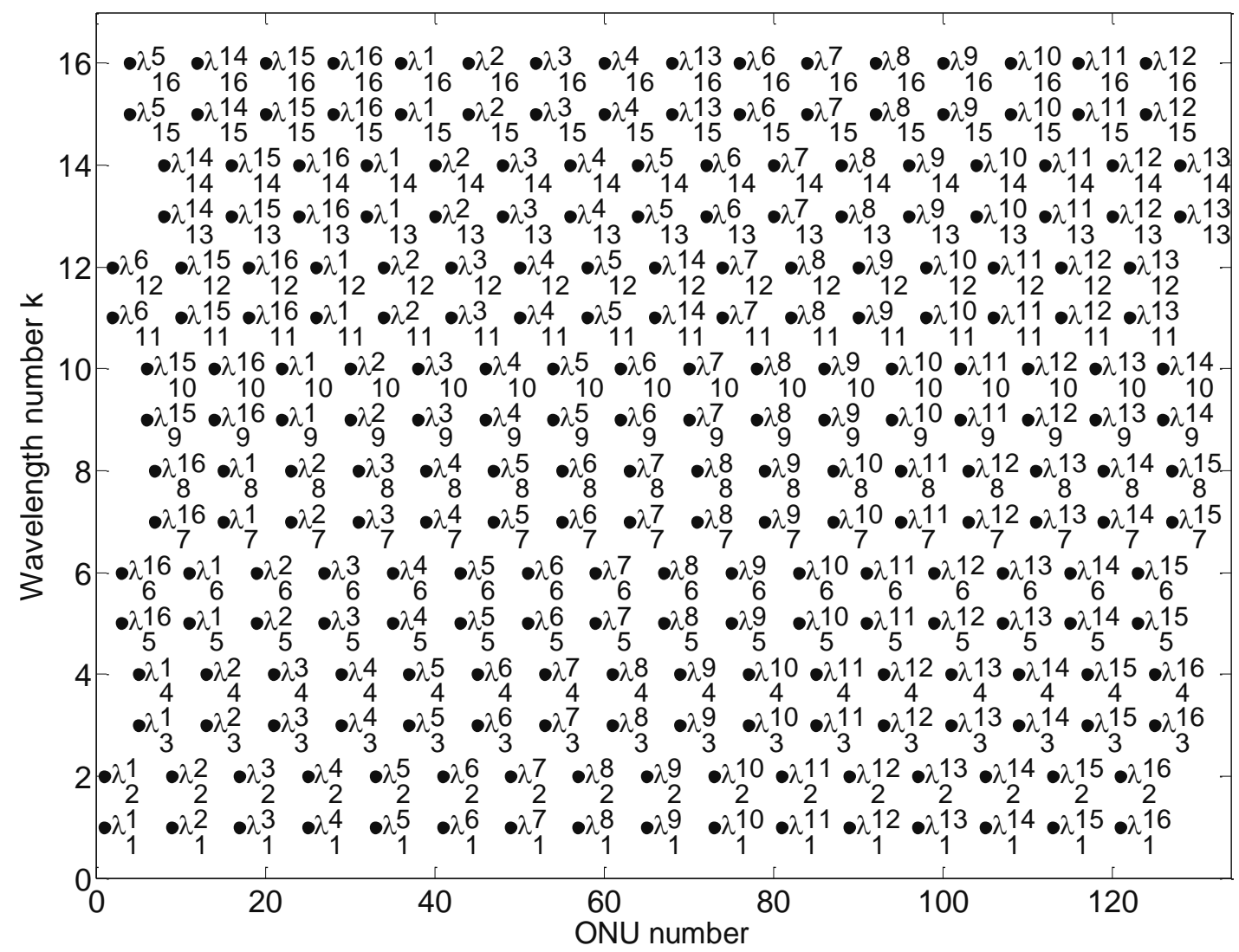

Figure 4.18: Connectivity map of the three-stage architecture from Figure 4.16 with increased coarseness $\overrightarrow{\mathrm{c}}=[2,4,8]$. 


\subsection{Capacity Limits}

The goal of this section is to elicit the greatest possible network architecture in terms of the capacity limit and the maximum number of users due to technology constraints.

In this work, we consider only networks with a tree topology (see design rule (V)). From this, the highest density of number of wavelengths per fiber is at the root of the tree at interstage 1 . The division of the accessible optical spectrum $\Delta \mathrm{F}$ by the channel spacing $\Delta \mathrm{f}$ leads to the number of wavelengths per fibern $\mathrm{n}_{\omega}$, which can be reused on each port of the WR. Therefore, the capacity limit for a multi-stage WDM PON is then given by

$$
\mathrm{B}_{\mathrm{N}}=\mathrm{B}_{0} \cdot \mathrm{N}_{\omega}=\mathrm{B}_{0} \cdot \mathrm{M}_{1} \cdot \frac{\Delta \mathrm{F}}{\Delta \mathrm{f}}
$$

The capacity limit depends on the channel bitrate $\mathrm{B}_{0}$, the size of the WR in the first stage, the accessible optical spectrum and the channel spacing. According to the highest density of wavelengths per fiber in the first stage, the capacity limit in (5.62) is the same as in single-stage WDM PONs and can be seen in Table 3.2. Compared with a single-stage WDM PON, the number of users in a multi-stage WDM PON is $\mathrm{M}_{\mathrm{S}} \cdot \mathrm{N}_{\mathrm{S}}$ and therefore $\mathrm{N}_{\mathrm{s}}$ times greater than in single-stage networks.

With an example, the limits are demonstrated. We consider a WDM PON with $\frac{\Delta \mathrm{F}}{\Delta \mathrm{f}}=32, \mathrm{M}_{\max }=64$ and 128 users, each one with a number of wavelengths of 16 . Thus, the maximum number of wavelengths in the multi-stage WDM PON is $32 \cdot 64=2048$. In other words, the multiplication of the users $U$ with the number of wavelengths per user $\mathrm{U}_{\omega}$ does not exceed the number 2048. It must be pointed out that the usage of smaller wavelength spacings allows us to increase the usage of umpteen thousand wavelengths. But smaller numbers of $\Delta \mathrm{f}$ result in higher costs for lasers and WR.

As we can see in Figure 4.19, the network is realizable with a two-stage WDM PON. Regarding section 4.1, the network parameters are determined with $\overrightarrow{\mathrm{N}}=[1,2]$, $\overrightarrow{\mathrm{M}}=[64,64], \overrightarrow{\mathrm{I}}=[64,32]$ and $\overrightarrow{\mathrm{c}}=[1,2]$. 


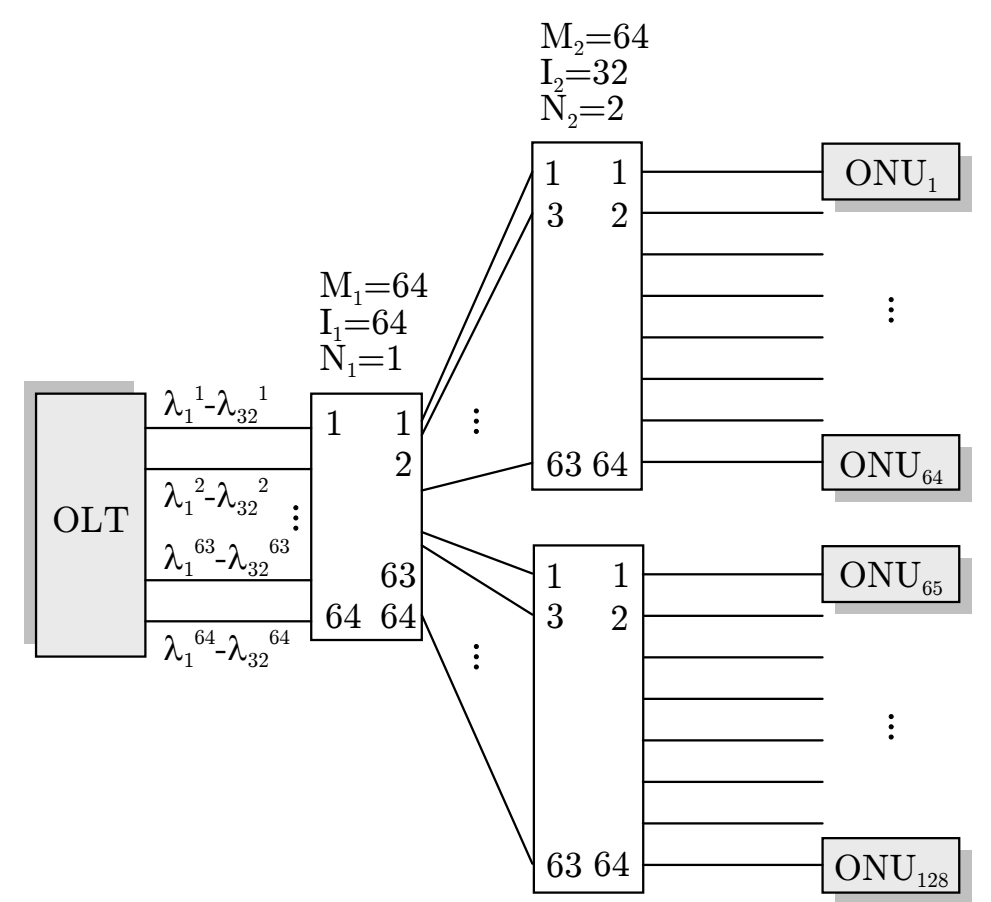

Figure 4.19: Example of a two-stage WDM PON architecture for $\mathrm{U}=128, \mathrm{U}_{\omega}=16, \mathrm{n}_{\omega}=32, \overrightarrow{\mathrm{c}}=[1,2]$, $\overrightarrow{\mathrm{N}}=[1,2], \overrightarrow{\mathrm{M}}=[64,64]$ and $\overrightarrow{\mathrm{I}}=[64,32]$.
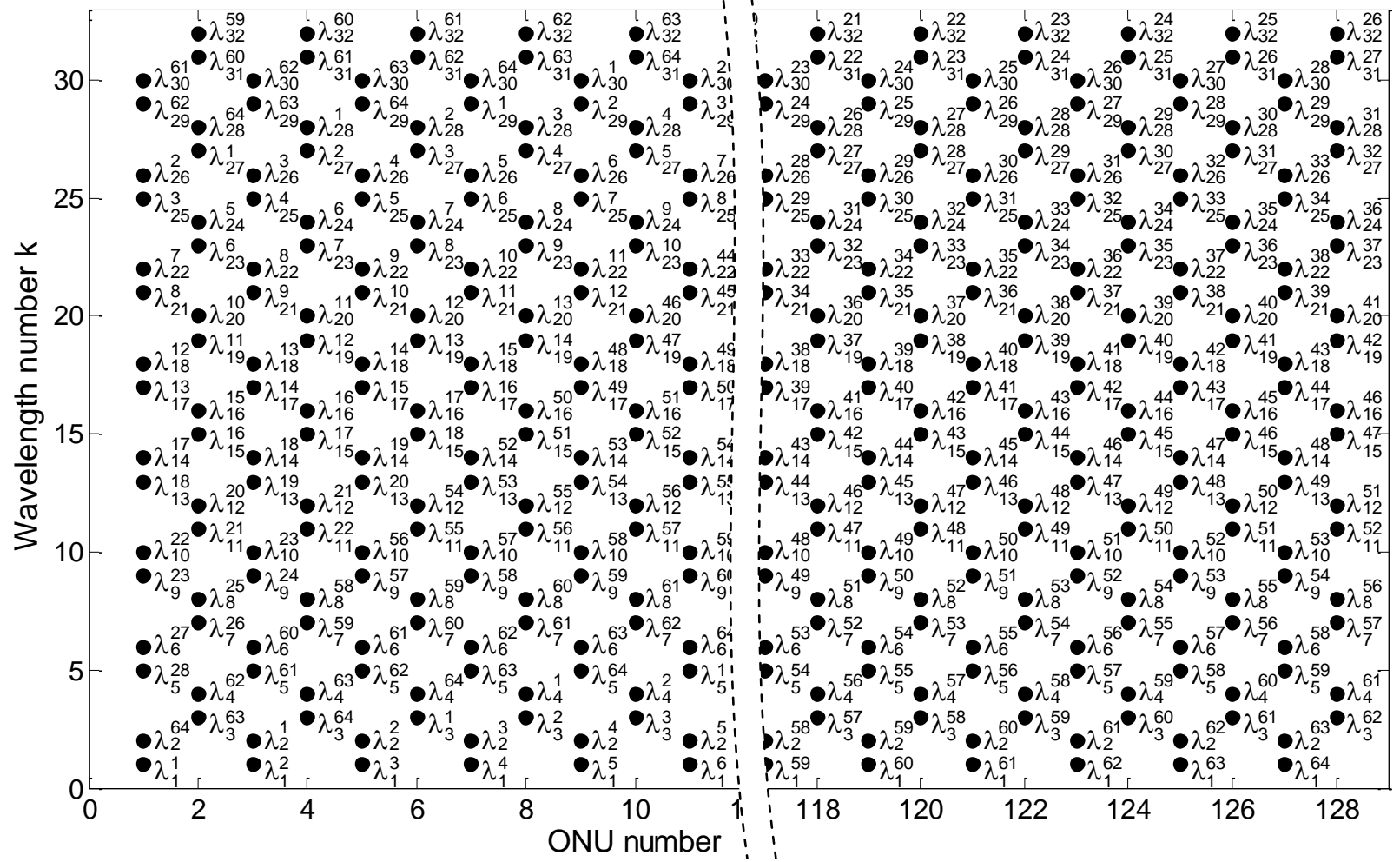

Figure 4.20: Connectivity map from the two-stage WDM PON in Figure 4.19. We realize that each ONU receives 16 wavelengths.

We recognize on the resulting connectivity map in Figure 4.20 that the objective 16 wavelengths for each of the 128 ONUs is fulfilled.

In the next section, the limitation on the basis of the power budget is shown. 


\subsection{Power budget}

A key factor in optical system design is the power budget. The power budget is essential for the correct performance of the optical network. The power budget was evaluated by performing an approximate calculation. For the calculation, the link length between the ONU and the OLT must be known. It is not an easy task to find this information, since, up to how, there have been no commercial installations of multistage WDM PONs.

[Mickelsson02] shows that with a link length of $1,500 \mathrm{~m}, 50 \%$ of all subscribers can be reached. In most countries, approximately $99 \%$ of all users are reachable inside a distance of $10 \mathrm{~km}$ and a link length of $20 \mathrm{~km}$ covers $>99.9 \%$ of all subscribers. Thus, we consider a maximal distance of $20 \mathrm{~km}$ for our power-budget calculations.

The following typical attenuation values are supposed:

- fiber attenuation: $0.2 \mathrm{~dB} / \mathrm{km}$

- max. fiber length: $20 \mathrm{~km}$

- connector loss: $0.2 \mathrm{~dB}$

- number of connectors: $8+2$ for each WR

- WR insertion loss: $4 \mathrm{~dB}$

- splice loss: 0.02dB

- number of splices: 6

On the basis of typical transceivers for Gigabit-Ethernet with a bitrate of $1.25 \mathrm{Gbit} / \mathrm{s}$, the following performance values are used [DeCusatis02, p. 159 et seq.]:

- power of the sender per wavelength: $0 \mathrm{dBm}$

- receiver sensitivity: $-30 \mathrm{dBm}$.

In Figure 4.21 the calculated values for the system margin for a multi-stage WDM PON is shown.

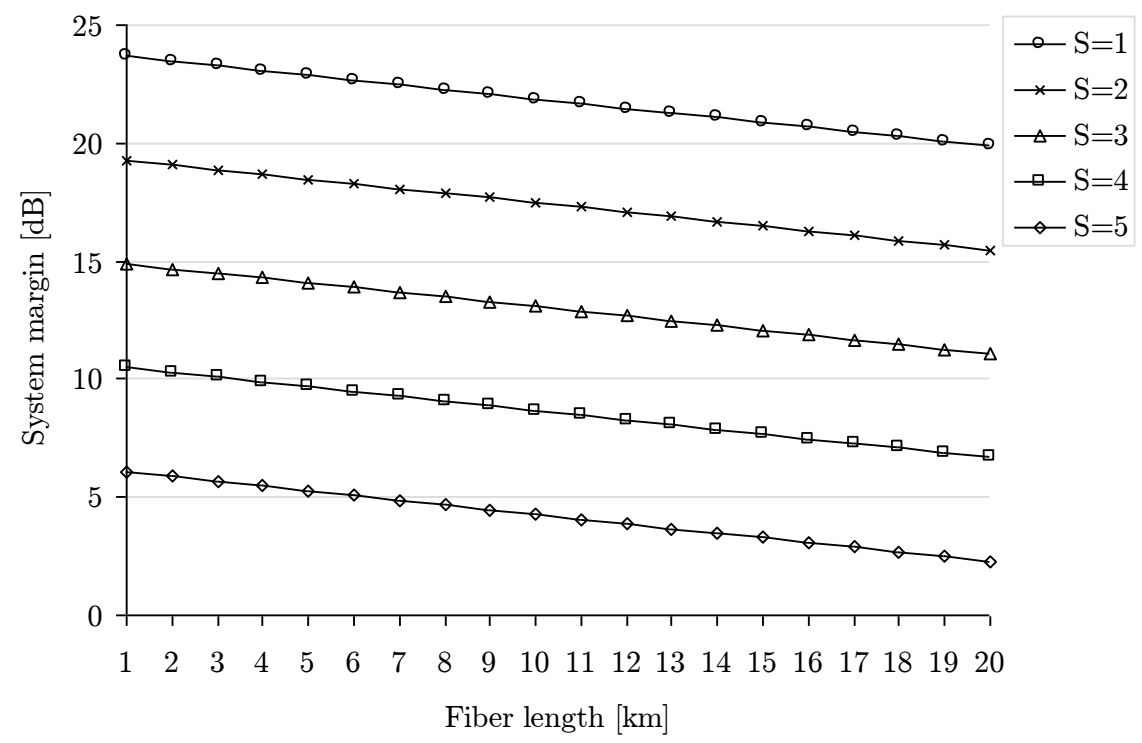

Figure 4.21: System margin of a multi-stage WDM PON in dependence of the fiber length as a function of the number of stages with an optical power at the sender of $0 \mathrm{dBm}$ and a receiver sensitivity of $-30 \mathrm{dBm}$. 
By means of the Figure 4.21 the typical system margin of $3 \mathrm{~dB}$ can be adhered to a 4 stage WDM PON independent of the fiber length. For a 5 stage WDM PON, the power budget must be optimized (optical power at the sender, receiver sensitivity, ...) for fiber lengths greater than $15 \mathrm{~km}$. It must be stated that an optimization of the optical power budget leads to lower costs.

It could be shown that for a multi-stage WDM PON of up to a number of 4 stages, the typical system margin of $3 \mathrm{~dB}$ can be satisfied.

On the basis of the methods described in chapters 3 and 4 , a techno-economic evaluation can be implemented. The goal of the techno-economic evaluation in the next chapter is to identify those architectures which minimize the cost. 


\section{Techno- Economic Evaluations}

7 his chapter discusses two important considerations: technological and economical. The goal is to identify those architectures that are technologically feasible while simultaneously minimizing costs. For a real deployment, the position of the wavelength router (WR) is a topic to further minimize costs and is researched in the last part of this chapter.

\subsection{Introduction}

In most cases of multistage WDM PONs, the technology has worked but the economics has not. Thus, it is important to examine the economical aspects. Thousands of different network architectures are technologically possible (see section 5.2). The objective of this techno- economic evaluation is to identify those architectures that minimize costs and are economically feasible [Schussmann06, Schussmann07a, Schussmann07c]. Generally in optical networks, the costs for digging, ducts, fibers is an integral part of the total costs. These costs can be minimized with a cleverly positioned WR. Our evaluations of the WRs position allow for a better understanding of this influencical parameter and can be used as a decision support system to minimize the costs.

In the past, many economic case studies have been published [Bell96, Bell98, Prat02, TERA, TONIC]. Each analysis uses a different model to identify the fiber length, ducts, etc. and different cost functions to determine those architectures that minimize the costs. In general, techno- economic evaluations can be performed at different levels as shown in Table 5.1.

All listed modeling levels have their own assets and drawbacks. The focus of our technoeconomic evaluations is to compare a various numbers of different architectures to find those architectures which minimize costs. In this case, the basic modeling level is the best choice despite the drawback of large margins of errors. A highly detailed modeling level has limited margins of errors but must manipulate a large amount of data which is dependent on the proper geographic data and is highly complex [Bachhiesl03]. 


\begin{tabular}{|c|c|c|c|}
\hline $\begin{array}{c}\text { Modeling } \\
\text { level }\end{array}$ & Method & Advantages & Disadvantages \\
\hline Basic & - Average length & $\begin{array}{l}\text { - Fast results } \\
\text { - Convenient to compare } \\
\text { different technologies }\end{array}$ & - Large margins of errors \\
\hline Detailed & $\begin{array}{l}\text { - Average length } \\
\text { and customer } \\
\text { densities }\end{array}$ & $\begin{array}{l}\text { - Good assessment for the } \\
\text { modeled area } \\
\text { - Convenient to compare } \\
\text { different technologies }\end{array}$ & - Medium margins of errors \\
\hline $\begin{array}{l}\text { Highly } \\
\text { detailed }\end{array}$ & $\begin{array}{l}\text { - Using geographic } \\
\text { positions and } \\
\text { existing ducts } \\
\text { routes and roads }\end{array}$ & $\begin{array}{l}\text { - Limited margins of } \\
\text { errors }\end{array}$ & $\begin{array}{l}\text { - Reliant on the availability } \\
\text { of proper data } \\
\text { - Highly complex } \\
\text { - Manipulation of large } \\
\text { amounts of data }\end{array}$ \\
\hline
\end{tabular}

Table 5.1: Different levels of modeling the techno- economic evaluation for networks.

Our evaluations use high level models that capture the essence of equipment and physical constraints to derive an economic sense in a proposed network. The architectural choices will be supported by techno- economical evaluations. An investment cost study is made which shows the cost structure of automatically generated WDM PON architectures. Previous works often focused on the investment cost and operational expenses for different first mile solutions. This work will focus more on the comparison of network architectural options and influencing factors, which implies setting the focus on a comparison of the investment cost. Costs that have no effect on the comparison were left out (such as central office (CO) building, electricity, lasers, etc.).

\subsection{Generation of Network Architectures}

On the basis of the design conventions and constraints in chapter 3 and 4 the design tool is able to generate all practicable architectures automatically. This assists an understanding of multistage WDM PONs and to introduce new architectures.

With our design tool we are able to identify all possible parameter identifications that correspond to an IC or DC WDM PON. Our purpose is to find out which combinations of the number of stages, size of the WR, number of used inputs and the coarseness factor for IC and DC WDM PONs are technologically feasible and minimize the costs.

To determine these combinations, many optimization methods like linear integer programming or other methods can be used. The number of possible network architectures are relatively small that meet the conditions in subsection 4.1.1 (design conventions). Thus, we use another approach to solve the problem. We generate all the possible network architectures for a given number of users and for all possible numbers of wavelengths per user. Then we compare the costs and identify the optimal parameter choice for a given number of users and wavelengths per user. 
Our design tool is composed of four parts (see Figure 5.1). The first module generates all combinations of $\overrightarrow{\mathrm{M}}$ and $\overrightarrow{\mathrm{N}}$ in dependence of $\mathrm{S}, \mathrm{M}_{\min }, \mathrm{M}_{\max }$ and $\mathrm{N}_{\max }$. In this work, the parameter $\mathrm{S}$ is chosen between 1 and 5 on the strength of the power budget consideration in section 4.4. Another required constraint is the size of the WR. At the moment, due to the technological constraints in the production process, a maximum size of the WR of $\mathrm{M}_{\max }=64$ is commercially available which is used to limit the number of architectures. We demarcate the number of architectures significantly when the minimum size of a WR is restricted to $\mathrm{M}_{\min }=4$. WR sizes smaller than 4 (e.g. $2 \times 2 \mathrm{WR}$ ) do not have much practical significance and lead to too small remote nodes. The number of WRs increases stage by stage due to the design convention (V). Thus, the highest number of WRs is in the last stage. The maximum number of WRs in one stage is limited to 64 . As a consequence, the maximum number of users in this work can be determined by $64 \times 64=4,096$ users.

The second module generates all vectors of $\overrightarrow{\mathrm{I}}$ and $\overrightarrow{\mathrm{c}}$ (for both IC- and DC WDM PON architectures) in dependence of $\mathrm{U}_{\omega}$ and $\mathrm{n}_{\omega}$ and then it moves through all the generated architectures from the first module. Those architectures that do not fulfill the design conventions will be excluded. Within the third module, the costs for all generated architectures are calculated. With the last module, architectures and costs can be analyzed. Two examples of architectures generated by the design tool are illustrated in Figure 5.3 for an IC WDM PON and for a DC WDM PON in Figure 5.4.

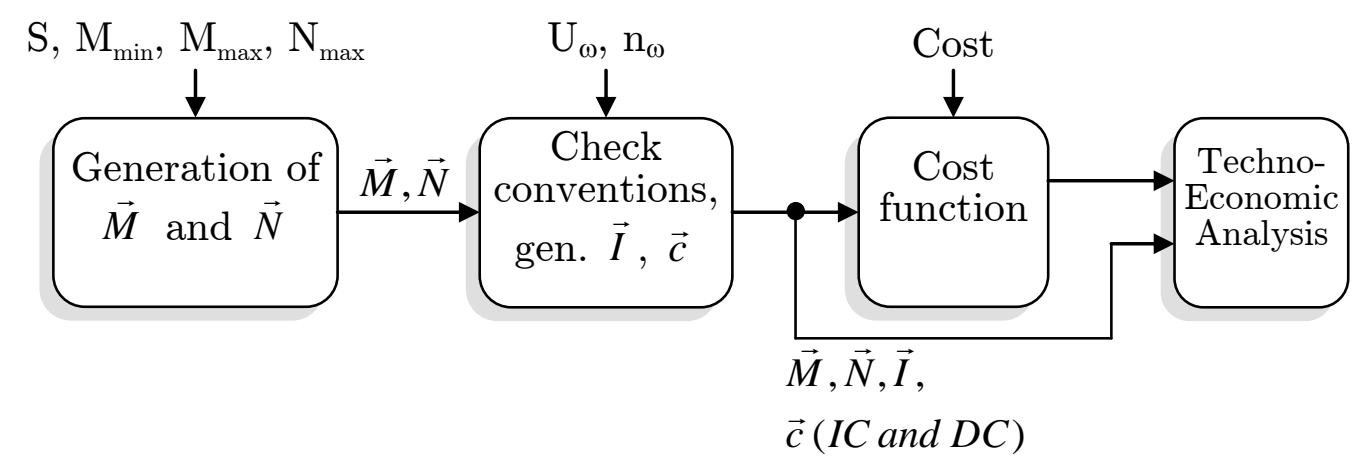

Figure 5.1: Flowchart of the functionality of the network design tool for single and multistage IC and DC WDM PONs.

In order to verify our tool and to estimate the number of possible architectures for a given parameter configuration, considerations to both questions are explained in the next paragraph.

Starting with the number of theoretically possible architectures, without including the design convention, the number of architectures can be calculated by the well known equation of variations with repetitions

$$
\mathrm{V}_{\mathrm{n}}^{(\mathrm{k})}=\mathrm{n}^{\mathrm{k}}
$$

where $\mathrm{k}$ is a selection from $\mathrm{n}$ different elements.

As a consequence, the number of variations for $\mathrm{N}$ is $\mathrm{n}^{\mathrm{S}-1}$ ( $\mathrm{S}-1$ due to the design convention (II) in which $\mathrm{N}_{1}=1$ ) and for $\mathrm{M}$ it is equal to $\mathrm{n}^{\mathrm{S}}$. The maximum number of $\mathrm{N}$ 
is chosen at 64 which results in an $\mathrm{n}$ of $7\left(\mathrm{~V}_{7}{ }^{(\mathrm{S}-1)}\right)$. The size of the WR is limited between 4 and 64 and will lead to an $\mathrm{n}$ of $5\left(\mathrm{~V}_{5}{ }^{(\mathrm{S})}\right)$. From this it follows that all theoretical variations can be calculated simply by multiplication of $\mathrm{V}_{7}^{(\mathrm{S}-1)}$ and $\mathrm{V}_{5}{ }^{(\mathrm{S})}$ (Table 5.2).

\begin{tabular}{rrrrrr}
\hline $\mathrm{S}$ & $\mathrm{V}_{7}{ }^{(\mathrm{S}-1)}$ & $\mathrm{V}_{5}{ }^{(\mathrm{S})}$ & $\mathrm{V}_{7}^{(\mathrm{S}-1)} \cdot \mathrm{V}_{5}{ }^{(\mathrm{S})}$ & $\mathrm{n}_{\omega} \rightarrow \infty \mathrm{IC}, \mathrm{U}_{\omega}=1$ & $\mathrm{n}_{\omega} \rightarrow \infty \mathrm{DC}, \mathrm{U}_{\omega}=1$ \\
\hline 1 & 1 & 5 & 5 & 5 & 5 \\
2 & 7 & 25 & 175 & 80 & 80 \\
3 & 49 & 125 & 6,125 & 782 & 755 \\
4 & 343 & 625 & 214,375 & 2,888 & 2,821 \\
5 & 2,401 & 3,125 & $7,503,125$ & 4,030 & 3,999 \\
\hline Sum & 2,801 & 3,905 & $7,723,805$ & 7,785 & 7,660 \\
\hline
\end{tabular}

Table 5.2: Number of theoretical and practical multistage WDM PON architectures to verify if our tool works properly. Theoretically 7,723,805 different architectures are possible. Due to the design conventions, only a fraction of the number of architectures is feasible. For an IC WDM PON, 7,785 possible architectures and 7,660 architectures are possible for a DC WDM PON.

Due to the restrictions of the design conventions, the maximum number of architectures (theoretical) cannot be reached. The number of architectures, in regards to the design conventions, additionally depends on the number of wavelength per user $\mathrm{U}_{\omega}$, the maximum number of wavelengths per fiber $n_{\omega}$ and on the number of stages $\mathrm{S}$. The maximum number of architectures realizable can be calculated with our design tool with the parameters $\mathrm{U}_{\omega}=1$ and $\mathrm{n}_{\omega} \rightarrow \infty$ (last two columns in Table 5.2). The maximum number of architectures in dependence of $\mathrm{S}$ generated by the design tool is shown in the last two columns in Table 5.2 for IC and DC WDM PONs.

The number of possible IC and DC architectures is different. Many simulations show us that the number of possible architectures of the type DC can be less than the architectures based on the IC type. On the basis of simulations of all architectures we found that in networks with the IC type, the coarseness factor can be less than 1 . Because of the design convention (IV), a coarseness factor less than 1 is not allowed in any stage of the multistage WDM PON. Therefore, the number of possible DC WDM PONs can be less than the number of possible IC WDM PONs (see Figure 5.2). 


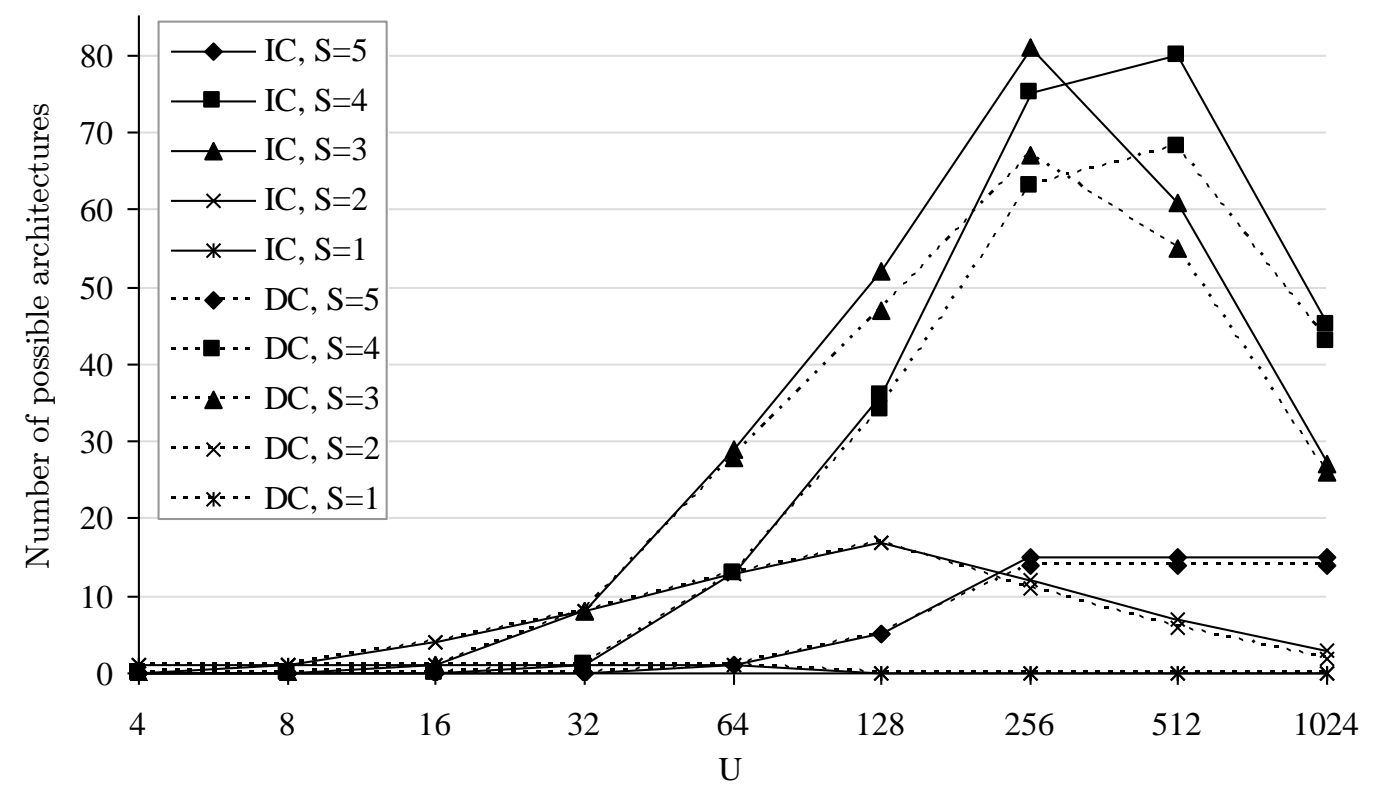

Figure 5.2: Example of the maximum number of multistage IC and DC WDM PON architectures in dependence from the number of users $\mathrm{U}$ with $\mathrm{S}$ between 1 and $5, \mathrm{n}_{\omega}=64, \mathrm{U}_{\omega}=2, \mathrm{M}_{\min }=4, \mathrm{M}_{\max }=64$, $\mathrm{N}_{\max }=64$. In summary, 1199 different number of architectures are possible.

The design tool automatically calculates the number of possible architectures. The number of possible architectures depends on $\mathrm{S}, \mathrm{n}_{\omega}, \mathrm{U}_{\omega}, \mathrm{M}_{\min }, \mathrm{M}_{\max }$ and $\mathrm{N}_{\max }$. As shown in the example in Figure 5.2, the number of possible architectures rises with the number of users. From this point in dependence of $\mathrm{S}$, the number of possible architectures remains constant or declines. This means that each curve in Figure 5.2 has its own maximum. This maximum is recommended because it allows a high degree of freedom. This degree of freedom results in a higher number of minimum cost architectures and allows the choice of those architectures that best match the conditions in the real world. In the case in Figure 5.2, the maximum possible architectures are of the IC type with $\mathrm{S}=3$ and 4 for an $\mathrm{U}$ with 256 and 512 respectively.

An example of architectures generated by the design tool is illustrated in Figure 5.3 for an IC WDM PON and in Figure 5.4 for a DC WDM PON. Both examples show the number of WRs in each stage, the size of the WR and the coarseness factor. For multiple asterisks in one point, they are summarized to a single asterisk sign. In Figure 5.3, each architecture has rising arrows which characterize the increasing coarseness factor in each stage such as an IC WDM PON. Declining arrows like in Figure 5.4 characterize DC WDM PON architectures. 


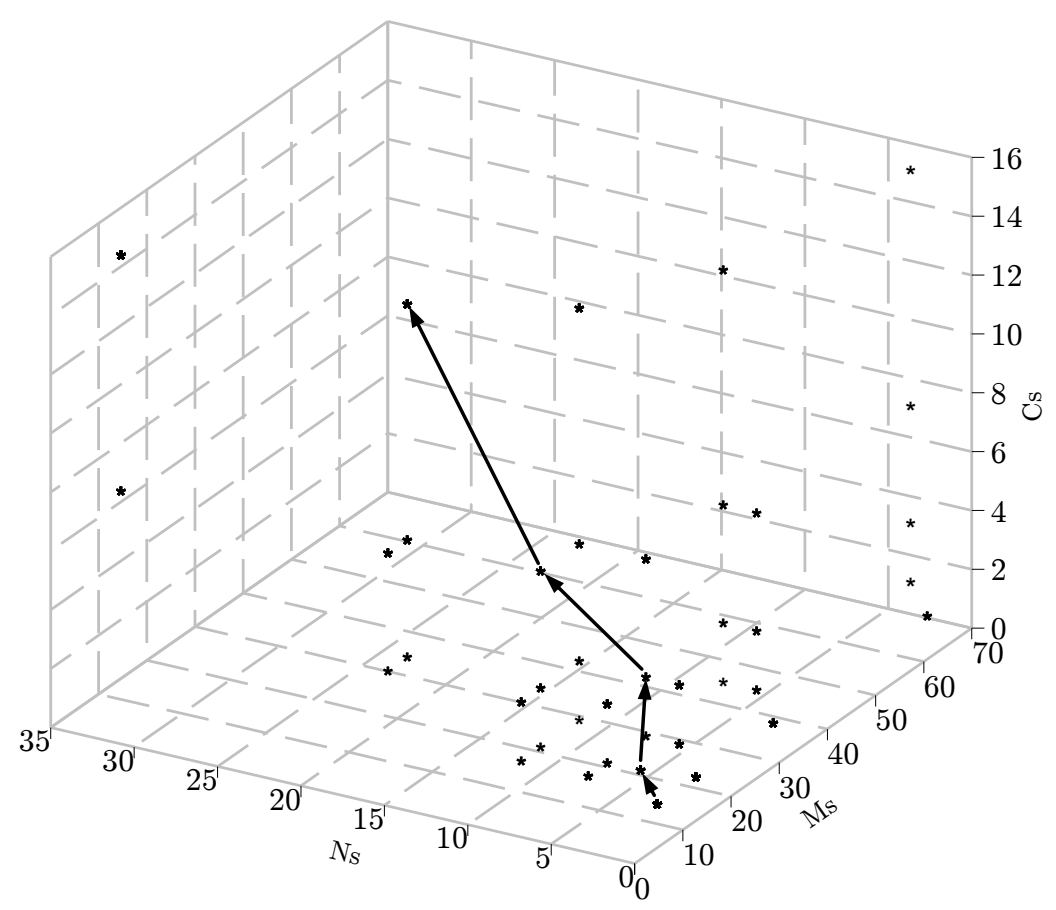

Figure 5.3: Generated architectures of the type IC WDM PON with $\mathrm{U}=128, \mathrm{U}_{\omega}=2, \mathrm{n}_{\omega}=64, \mathrm{M}_{\min }=4$ and $\mathrm{M}_{\max }=64$. The arrows identify the architecture: $\overrightarrow{\mathrm{c}}=[1,2,4,8,16], \overrightarrow{\mathrm{M}}=[8,8,8,8,8], \overrightarrow{\mathrm{N}}=[1,2,4,8,16]$ and $\overrightarrow{\mathrm{I}}=[4,4,4,4,4]$.

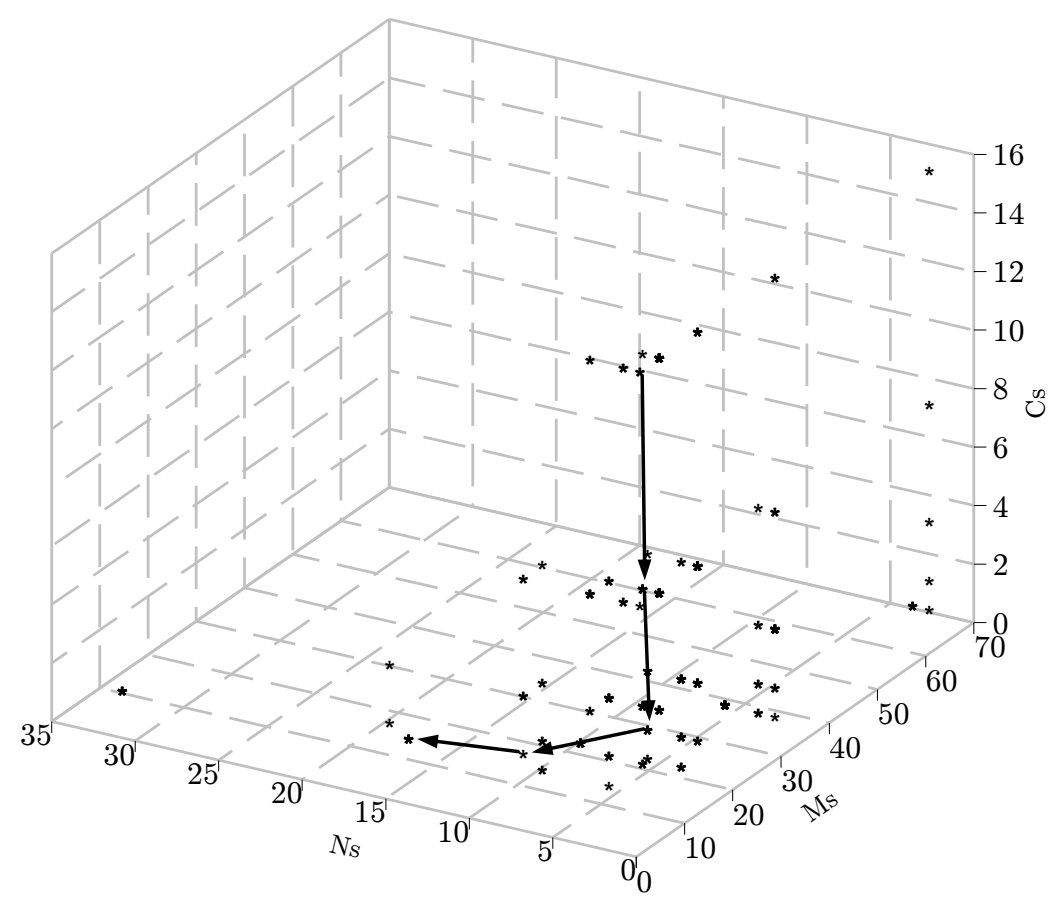

Figure 5.4: Generated architectures of the type DC WDM PON with $\mathrm{U}=128, \mathrm{U}_{\omega}=2, \mathrm{n}_{\omega}=64, \mathrm{M}_{\min }=4$ and $\mathrm{M}_{\max }=64$. The arrows identify the architecture: $\overrightarrow{\mathrm{c}}=[16,8,4,2,1], \overrightarrow{\mathrm{M}}=[8,8,8,8,8], \overrightarrow{\mathrm{N}}=[1,2,4,8,16]$ and $\overrightarrow{\mathrm{I}}=[4,4,4,4,4]$.

In both examples, a concentration in the direction of smaller numbers of $\mathrm{M}_{\mathrm{s}}$ and $\mathrm{N}_{\mathrm{s}}$ is obvious. Therefore, it is important to minimize the costs for smaller WR sizes due to their higher frequency relating to larger WR sizes. 


\subsection{Cost Performance}

This section deals with the cost functions (see also Figure 5.1) to determine the costs of the entire WDM PON. Accordingly, the performance analysis with its influencing parameters is discussed and the minimum cost architectures for realistic chosen network parameters are illustrated.

\subsubsection{Cost Function}

Our design tool calculates the realizable architectures in dependence from $\mathrm{U}_{\omega}, \mathrm{n}_{\omega}, \mathrm{M}_{\min }$, $\mathrm{M}_{\max }$, $\mathrm{S}$ and $\mathrm{N}_{\max }$. The objective is to identify architectures that minimize the costs. To compare all generated architectures by the design tool, three distinct cost functions are defined. Only those costs are considered which allow a comparison of different architectures. In the economical evaluations, we have considered the costs for commercially available devices at the end of 2006.

To compare the variety of architectures, the cost function consists of three distinct cost portions. The first one includes the cost of the WR. The WR cost depends basically on the number of ports and so far as possible is independent from the coarseness factor (Table 5.3). The coarseness factor of the WR is determined by the individual design of the star couplers and the integrated waveguides. The number of ports of the WR directly concerns its physical dimension. The reported costs in Table 5.3 have been provided by a manufacturer. It is appropriate to expect a drop in costs of WR manufacturing, thus the production volumes should increase. This scenario is likely to happen in the future as these devices will become more and more accepted as devices to set-up optical networks.

Cost evolutions of devices are defined by attaching them to certain learning curve classes and volume classes. Learning curves are based on the fact that every time you increase the number of units that you make, you will experience a predictable decrease in the time that it takes you to make the next unit. This production phenomenon in which each succeeding unit is easier to make has been proven. Mostly in the literature, the learning curve is a function of produced units [Miller02, pp. 47-48]. When we use cost prediction models in economic calculations, it is important to forecast the cost evolution as a function of time, not as a function of produced units. The volume class gives the manufactured volume as a function of time. In [Tele99, Olsen04, TERA] a number of volume classes are predefined based on data from the industry. The evaluation of the cost forecasts based on learning curve classes and volume classes can be made with the so called extended learning curve model [Olsen04]. However, the evaluation of cost forecasts is beyond the scope of this work. 
The second cost function take into acount the cable cost. The cable cost depends mainly on the number of fibers. The differences between them are significant. Thus, five different cable costs between 4 and 64 fibers are used in the economic analysis (Figure 5.4). This implies that each user is linked with four fibers. We suppose for simplicity that all the $I_{s}$ fibers in stage s are placed in one single cable.

The third cost function accounts for the installation cost (Table 5.4) [Tan97]. It contains the cost of the installation and of the trenching building operation. Partial cable reuse is not taken into account and therefore it would overestimate the cable and installation costs if networks with more PONs had to be constructed.

For calculating the cable and installation costs, link lengths of all the cable links in a multistage WDM PON must be known to calculate the total cost. This is not an easy task because there are no area-wide commercial multistage WDM PON installations today. Therefore, link lengths must be assumed based on more traditional networks. [Mickelsson02] shows that with a link length of 1,500m, $50 \%$ of all subscribers can be reached. Thus, the sum of the link lengths in a stage is always $1,500 \mathrm{~m}$ (Table 5.5). The length of the interstage links is also difficult to forecast. We assume a distribution as reported in Table 5.5. The impact of this assumption and the intention to find the optimal WR position is analyzed in section 5.4 .

It must be stated that our tool can be adopted for more comprehensive cost functions.

\begin{tabular}{c|cccccc}
\hline Number of & \multicolumn{7}{|c}{ Link number } \\
WR stages & 1 & 2 & 3 & 4 & 5 & $>5$ \\
\hline 1 & 900 & 600 & & & & \\
2 & 900 & 400 & 200 & & & \\
3 & 900 & 400 & 100 & 100 & & \\
4 & 900 & 400 & 100 & 50 & 50 & \\
$>4$ & 900 & 400 & 100 & 50 & 30 & 20 \\
\hline
\end{tabular}

Table 5.5: Link lengths in meters in multistage WDM PONs dependent on the number of WR stages. Links are numbered beginning at the $\mathrm{CO}$ and then going in the direction of the ONUs [Mickelsson02].

\subsubsection{Performance Analysis}

With our design tool, a detailed analysis of the cost evaluations for all architectures can be made. In a real deployment the multistage WDM PON needs to be adapted to the actual user density and adjusted to the WDM PON architecture if needed.

In this work we consider the architectures with $\mathrm{U}=128, \mathrm{U}_{\omega}=2, \mathrm{n}_{\omega}=64, \mathrm{M}_{\min }=4$ and $\mathrm{M}_{\max }=64$. These numbers derive from a technological perspective and are freely chosen. 
The small number of wavelengths per user is chosen to illustrate an applicable scenario. This is a likely scenario in a real deployment to start with a small number of wavelengths per user. Then it can be gradually increased with the user requirements. The values for $M_{\min }$ and $M_{\max }$ seem quite feasible for the present and the near-future WR technology.

The data obtained from the cost analysis function were first grouped by the number of stages $\mathrm{S}$, and then each group is ordered by increasing costs per user. To get a clearly arranged view, the resulting costs are numbered in ascending order beginning by zero and act as an index, called architecture index (Figure 5.5 - Figure 5.9). It should be noted that architectures with an architecture index of zero represents the WDM PON with the best cost performance.

In our cost evaluations, the costs per user are always reported and not the absolute cost values, to make the values comparable. But it must be stated that with a rising number of stages the number of potential users tends to increase. This fact tends to lower costs per user. This concept holds if all users are connected to the network and therefore share the costs for WR, fiber and so on.

The costs for WR per user are illustrated in Figure 5.5. This cost tends to increase with the number of stages. But this is not true because some architectures having two stages are more expensive than others with three stages. The increasing costs for a higher number of stages is the reason for the higher number of WRs needed. It must be noted that architectures with an architecture index of zero represent WDM PONs with the best cost performance.

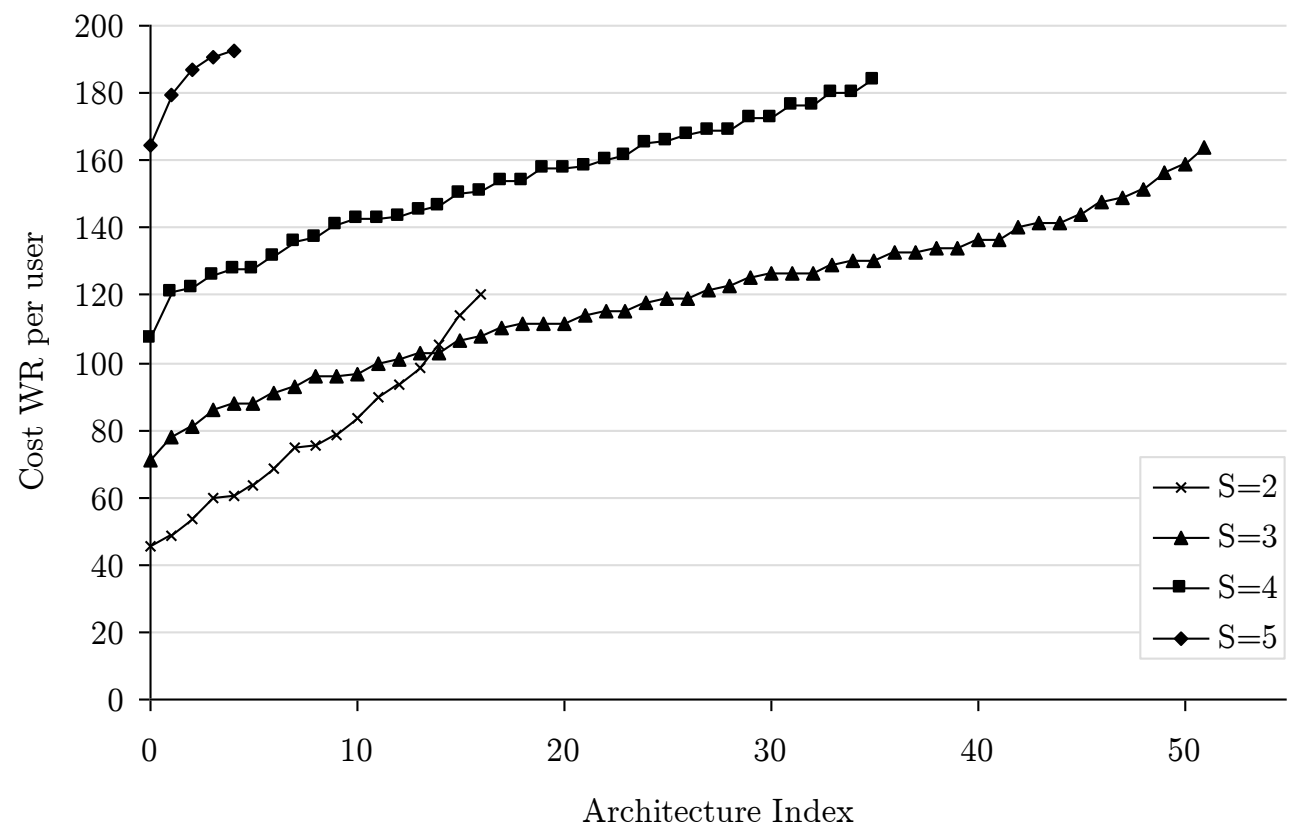

Figure 5.5: WR costs per user for the architectures received from the design tool with $\mathrm{U}=128, \mathrm{U}_{\omega}=2$, $\mathrm{n}_{\omega}=64, \mathrm{M}_{\min }=4$ and $\mathrm{M}_{\max }=64$. Architectures are ordered by increasing costs.

In Figure 5.6, the cost for cable per user tends to decrease with the number of stages. This behavior is due to the fact that by adding more stages, the link lengths at the lower stages are shorter, and therefore the costs are more shared in the lower stages. 
Comparing the costs for WR per user and cable per user, a cost trend in the opposite direction concerning the number of stages is obvious.

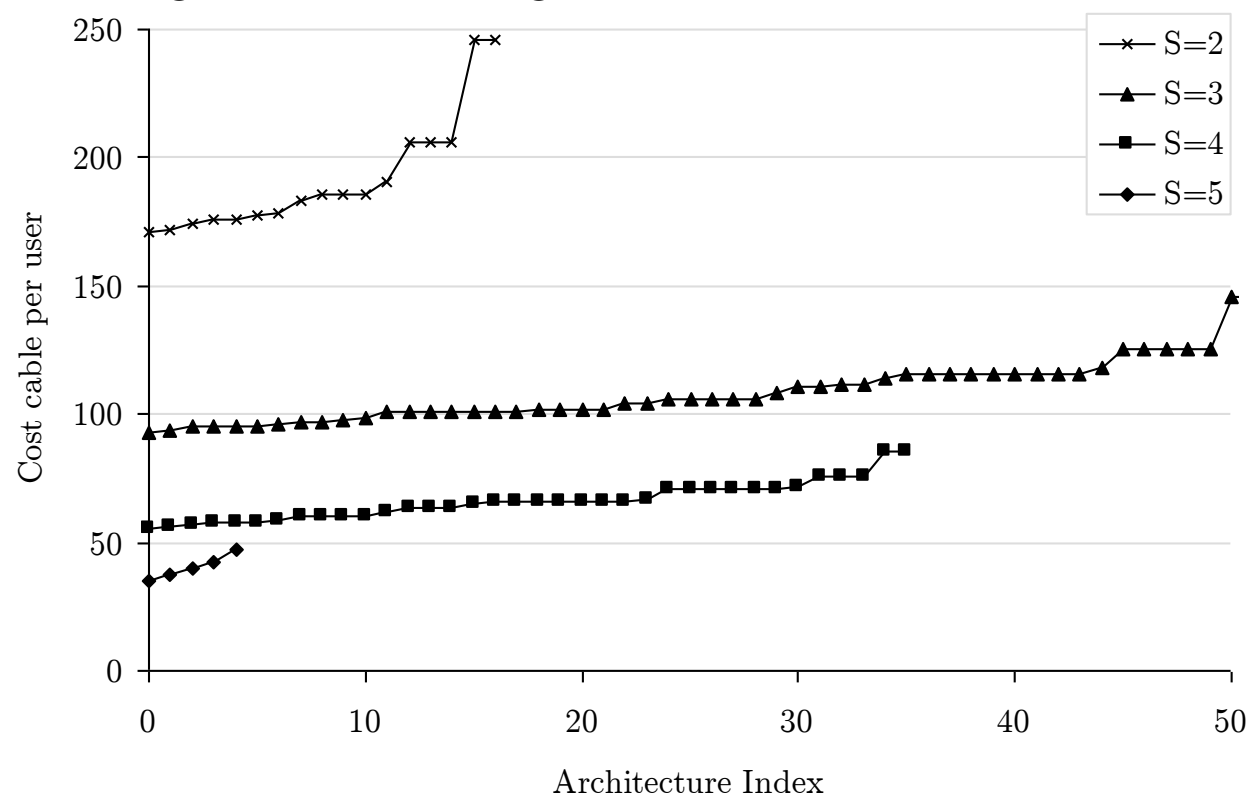

Figure 5.6: Cable costs per user for the architectures received from the design tool with $U=128, \mathrm{U}_{\omega}=2$, $\mathrm{n}_{\omega}=64, \mathrm{l}_{\max }=1,500 \mathrm{~m}, \mathrm{M}_{\min }=4$ and $\mathrm{M}_{\max }=64$. Architectures are ordered by increasing costs.

The installation costs per user is depicted in Figure 5.7. Analog to the cable costs, the installation costs tend to decrease with the higher number of stages although the installation costs are independent from the number of fibers in a cable.

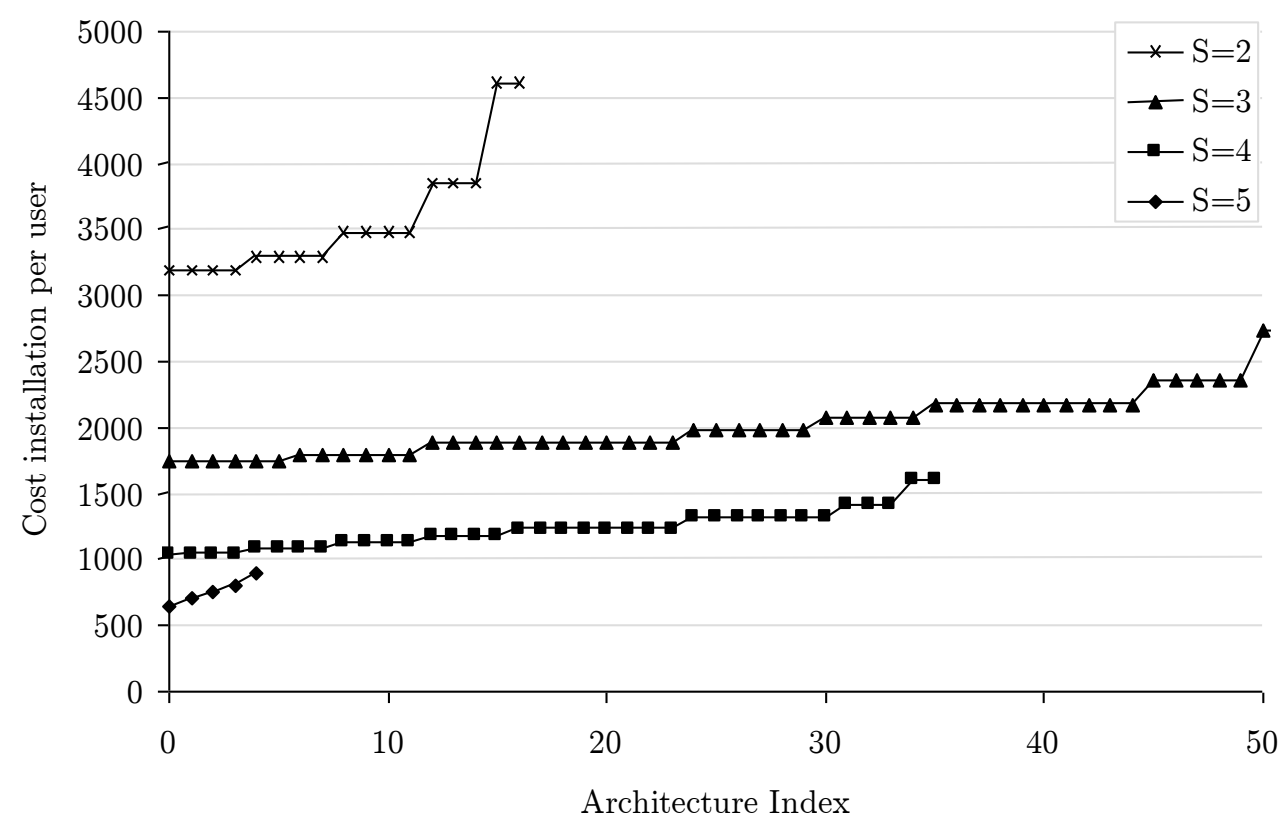

Figure 5.7: Installation costs per user for the architectures received from the design tool with $\mathrm{U}=128$, $\mathrm{U}_{\omega}=2, \mathrm{n}_{\omega}=64, \mathrm{l}_{\max }=1,500 \mathrm{~m}, \mathrm{M}_{\min }=4$ and $\mathrm{M}_{\max }=64$. Architectures are ordered by increasing costs.

The sum of the three cost components is shown in Figure 5.8. The best cost performance architectures are those with $\mathrm{S}=5$ and then closely followed by those with $\mathrm{S}=4$. This is the result of the relatively higher cost of cable and installation compared with the WR cost. 


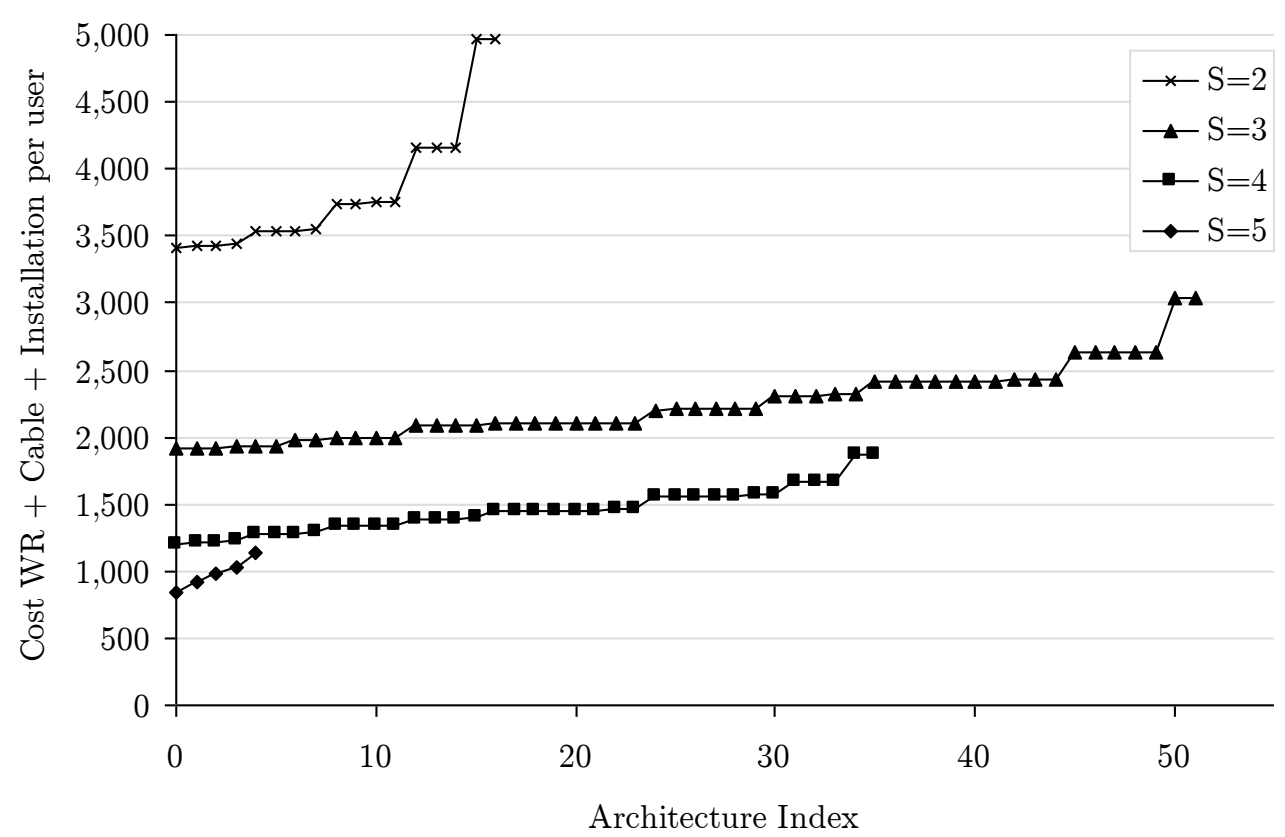

Figure 5.8: Total costs per user for the architectures received from the design tool with $\mathrm{U}=128, \mathrm{U}_{\omega}=2$, $\mathrm{n}_{\omega}=64, \mathrm{l}_{\max }=1,500 \mathrm{~m}, \mathrm{M}_{\min }=4$ and $\mathrm{M}_{\max }=64$. Architectures are ordered by increasing costs.

If the network operator can partially reuse an already installed plant by upgrading it to a WDM PON, only the WR and cable cost must be considered (see Figure 5.9). In this case, the best performance architectures are those with $\mathrm{S}=3$ closely followed by those with $\mathrm{S}=4$.

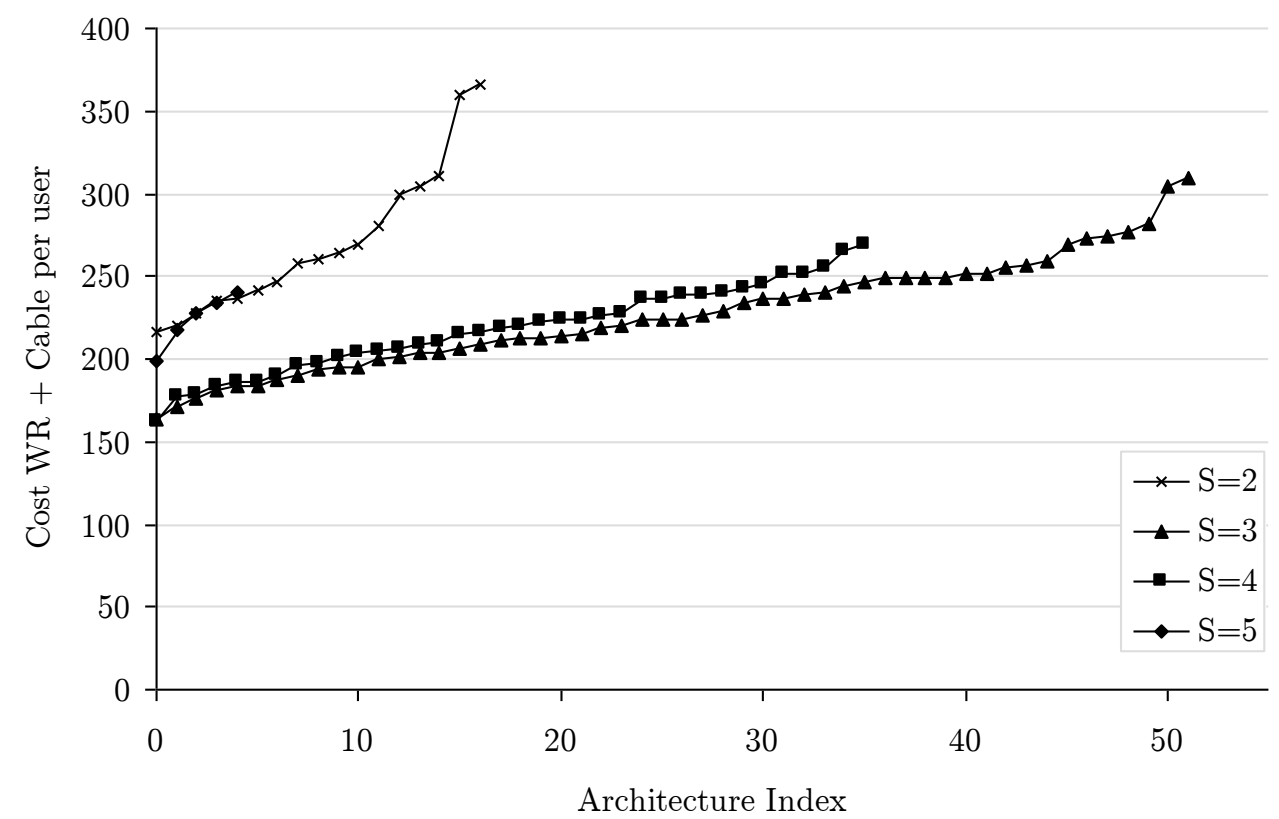

Figure 5.9: WR and cable costs per user for the architectures received from the design tool with $\mathrm{U}=128$, $\mathrm{U}_{\omega}=2, \mathrm{n}_{\omega}=64, \mathrm{l}_{\max }=1,500 \mathrm{~m}, \mathrm{M}_{\min }=4$ and $\mathrm{M}_{\max }=64$. Architectures are ordered by increasing costs. 
As stated before, the minimum cost architectures are those with the architecture index 0. For a given parameter configuration, only one minimum cost architecture exists. Those best cost performance architectures with the parameter configuration $U=(32,64$, 128, 256, 1024), $\mathrm{n}_{\omega}=64, \mathrm{U}_{\omega}=64, \mathrm{M}_{\min }=4$ and $\mathrm{M}_{\max }=64$ are depicted in Table 5.6. The data in Table 5.6 are first sorted in ascending order by the number of users and secondly sorted in ascending order by the costs per user.

\begin{tabular}{|c|c|c|c|c|c|c|c|}
\hline $\mathrm{U}$ & $\mathrm{S}$ & $\overrightarrow{\mathrm{M}}$ & $\overrightarrow{\mathrm{N}}$ & $\overrightarrow{\mathrm{I}}$ & $\overrightarrow{\mathrm{c}}(\mathrm{IC})$ & $\overrightarrow{\mathrm{c}}(\mathrm{DC})$ & Cost per user $[€]$ \\
\hline 32 & 4 & {$[4,4,4,4]$} & {$[1,2,4,8]$} & {$[1,2,2,2]$} & {$[1,4,8,16]$} & {$[16,4,2,1]$} & 2,212 \\
\hline 32 & 3 & {$[4,4,8]$} & {$[1,2,4]$} & {$[1,2,2]$} & {$[1,4,8]$} & {$[16,4,1]$} & 2,739 \\
\hline 32 & 2 & {$[4,16]$} & {$[1,2]$} & {$[1,2]$} & {$[1,4]$} & {$[16,1]$} & 4,082 \\
\hline 32 & 1 & {$[32]$} & {$[1]$} & {$[1]$} & {$[1]$} & {$[1]$} & 9,979 \\
\hline 64 & 5 & {$[4,4,4,4,4]$} & {$[1,2,4,8,16]$} & {$[2,2,2,2,2]$} & {$[1,2,4,8,16]$} & {$[16,8,4,2,1]$} & 1,245 \\
\hline 64 & 4 & {$[4,4,4,8]$} & {$[1,2,4,8]$} & {$[2,2,2,2]$} & {$[1,2,4,8]$} & {$[16,8,4,1]$} & 1,536 \\
\hline 64 & 3 & {$[4,4,16]$} & {$[1,2,4]$} & {$[2,2,2]$} & {$[1,2,4]$} & {$[16,8,1]$} & 2,187 \\
\hline 64 & 2 & {$[4,32]$} & {$[1,2]$} & {$[2,2]$} & {$[1,2]$} & {$[16,1]$} & 3,641 \\
\hline 64 & 1 & {$[64]$} & {$[1]$} & {$[2]$} & {$[1]$} & {$[1]$} & 9,742 \\
\hline 128 & 5 & {$[8,8,8,8,8]$} & {$[1,2,4,8,16]$} & {$[4,4,4,4,4]$} & {$[1,2,4,8,16]$} & {$[16,8,4,2,1]$} & 849 \\
\hline 128 & 4 & {$[8,8,8,16]$} & {$[1,2,4,8]$} & {$[4,4,4,4]$} & {$[1,2,4,8]$} & {$[16,8,4,1]$} & 1,206 \\
\hline 128 & 3 & {$[8,8,32]$} & {$[1,2,4]$} & {$[4,4,4]$} & {$[1,2,4]$} & {$[16,8,1]$} & 1,910 \\
\hline 128 & 2 & {$[8,64]$} & {$[1,2]$} & {$[4,4]$} & {$[1,2]$} & {$[16,1]$} & 3,415 \\
\hline 256 & 5 & {$[16,16,16,16,16]$} & {$[1,2,4,8,16]$} & {$[8,8,8,8,8]$} & {$[1,2,4,8,16]$} & {$[16,8,4,2,1]$} & 638 \\
\hline 256 & 4 & {$[16,16,16,32]$} & {$[1,2,4,8]$} & {$[8,8,8,8]$} & {$[1,2,4,8]$} & {$[16,8,4,1]$} & 1,032 \\
\hline 256 & 3 & {$[16,16,64]$} & {$[1,2,4]$} & {$[8,8,8]$} & {$[1,2,4]$} & {$[16,8,1]$} & 1,764 \\
\hline 256 & 2 & {$[16,64]$} & {$[1,4]$} & {$[8,4]$} & {$[1,2]$} & {$[8,1]$} & 3,359 \\
\hline 512 & 5 & {$[32,32,32,32,32]$} & {$[1,2,4,8,16]$} & {$[16,16,16,16,16]$} & {$[1,2,4,8,16]$} & {$[16,8,4,2,1]$} & 518 \\
\hline 512 & 4 & {$[32,32,32,64]$} & {$[1,2,4,8]$} & {$[16,16,16,16]$} & {$[1,2,4,8]$} & {$[16,8,4,1]$} & 934 \\
\hline 512 & 3 & {$[32,32,64]$} & {$[1,2,8]$} & {$[16,16,8]$} & {$[1,2,4]$} & {$[8,4,1]$} & 1,710 \\
\hline 512 & 2 & {$[32,64]$} & {$[1,8]$} & {$[16,4]$} & {$[1,2]$} & {$[4,1]$} & 3,331 \\
\hline 1024 & 5 & {$[64,64,64,64,64]$} & {$[1,2,4,8,16]$} & {$[32,32,32,32,32]$} & {$[1,2,4,8,16]$} & {$[16,8,4,2,1]$} & 443 \\
\hline 1024 & 4 & {$[64,64,64,64]$} & {$[1,2,4,16]$} & {$[32,32,32,16]$} & {$[1,2,4,8]$} & {$[8,4,2,1]$} & 895 \\
\hline 1024 & 3 & {$[64,64,64]$} & {$[1,2,16]$} & {$[32,32,8]$} & {$[1,2,4]$} & {$[4,2,1]$} & 1,681 \\
\hline 1024 & 2 & {$[64,64]$} & {$[1,16]$} & {$[32,4]$} & {$[1,2]$} & {$[2,1]$} & 3,316 \\
\hline
\end{tabular}

Table 5.6: Minimum cost architectures with $\mathrm{U}=(32,64,128,256,512,1024), \mathrm{n}_{\omega}=64, \mathrm{U}_{\omega}=64, \mathrm{M}_{\min }=4$ and $\mathrm{M}_{\max }=64$.

In the next section, the ideal WR position in a multistage WDM PON in terms of minimizing the costs is discussed. 


\subsection{WR Position}

A topic to be considered in planning a multistage WDM PON architecture is the position of the WR. One of the main cost issues in a multistage WDM PON is the costs for the installed fiber that increase as the WR position is kept close to the OLT due to the higher fiber count. In the past, simple models have been used to determine the splitter position in a PON [Caviglia98], but no evaluations exist for a multistage WDM PON. Economical and technical considerations concerning the WR positions are evaluated. The aim of this section is to identify those WR positions that best minimize costs. To provide a better understanding, the influence of certain parameters are identified and shown. It must be stated that our tool can be easily generalized to identify the optimal positions of other network components such as passive splitters and so on.

\subsubsection{Network Architecture}

The architecture considered as a reference is depicted in Figure 5.10. The distance between the OLT and the ONUs are the same and limited to $l_{\max }$. The distance between all WRs in stage $\mathrm{s}$ and stage $\mathrm{s}+1$ is $l_{\mathrm{s}+1}$. The basic principle of the operation method is a virtual movement of all WRs in a stage. This virtual movement is done for each stage and for each combination, so that all combinations of virtual movements have been carried out. After each movement, the costs were calculated and documented. The documented data can then be analyzed to determine the optimal WR position to minimize the cost.

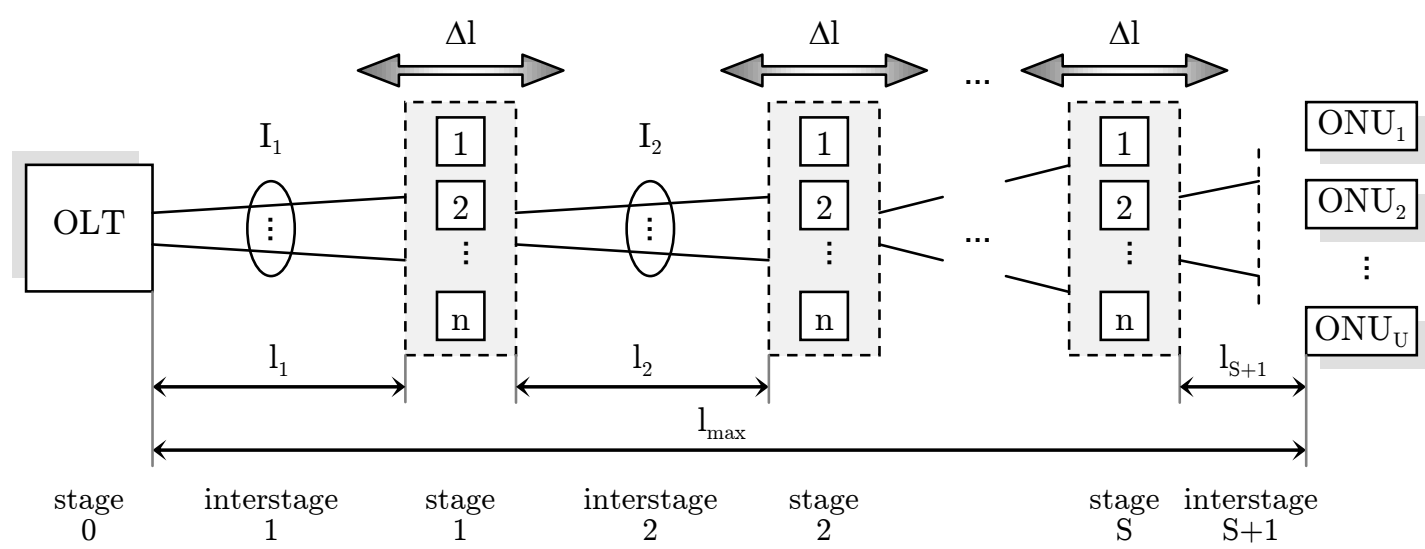

Figure 5.10: Architecture of the multistage WDM PON used by the calculation of the optimal WR position to minimize cable costs.

The simulation starts with the assumption that all WRs are located at the OLT in the central office. Thus, the length difference $l_{1}$ to $l_{\mathrm{S}+1}$ starts with 0 , whereas the length between the OLT and the ONU (in this case $l_{S+1}$ ) is $l_{\max }$. Next, all WRs in stage $\mathrm{S}$ are shifted by the length variation $\Delta \mathrm{l}$ towards the ONUs as long as they reach the ONUs. In each step, the costs are calculated. The shifting of the WR is made for all individual stages to identify all combinations possible within $1_{\max }$. 
The resolution of the length variation $\Delta \mathrm{l}$ and the length between the OLT and the ONU $1_{\max }$ can be freely chosen in the design tool. This is an important property to control the computational complexity and is done by choosing convenient $l_{\max } / \Delta \mathrm{l}$ ratios.

In Figure 5.11, the flowchart with its input and output parameters is shown. The first module generates all combinations of $l_{1} \ldots l_{S+1}$ and $U$ in dependence of $S, \Delta l, l_{\max }, \vec{N}, \vec{M}$ and $\overrightarrow{\mathrm{I}}$. The cost function determines the cost per user and can be expressed by the sum of all cost portions from interstage 1 up to interstage $\mathrm{S}+1$

$$
\text { Cost per user }=\frac{1}{U}\left[\left(\sum_{\mathrm{S}=1}^{\mathrm{S}} \mathrm{I}_{\mathrm{S}} \cdot \mathrm{N}_{\mathrm{S}} \cdot \mathrm{l}_{\mathrm{S}} \cdot \mathrm{C}_{\mathrm{f}}\left(\mathrm{I}_{\mathrm{S}}\right)\right)+\mathrm{U} \cdot \mathrm{l}_{\mathrm{S}+1} \cdot \mathrm{C}_{\mathrm{f}}\left(\mathrm{I}_{\mathrm{S}}\right)\right] \text {. }
$$

The term in the parenthesis represents the costs of all interstages except the interstage $\mathrm{S}+1$. The last term displays the costs between the stage $\mathrm{S}$ and the users. The expense factor $C_{f}\left(I_{s}\right)$ represents the fiber cost and is a function of $I_{s}$ regarding Table 5.4. The cable between the stage $\mathrm{S}$ and the users always contains 4 fibers as there is a minimal cost difference to a cable with 2 fibers.

The fiber cost per user is subject to the assumption that no digging and construction work are required. Therefore, the existence of ducts all along the path to install the cables has been assumed. This assumption does not impair the cost comparison significantly among different architectures, stages and WR positions because the digging costs are weakly dependant on the cable and fiber count and therefore on the WR position. For a complete cost comparison, all other costs (e.g. housing of the WR) must be taken into account.

The last module in Figure 5.11 represents the techno- economic analysis module in which all the generated data can be analyzed, e.g. to determine those WR positions that best minimize costs.

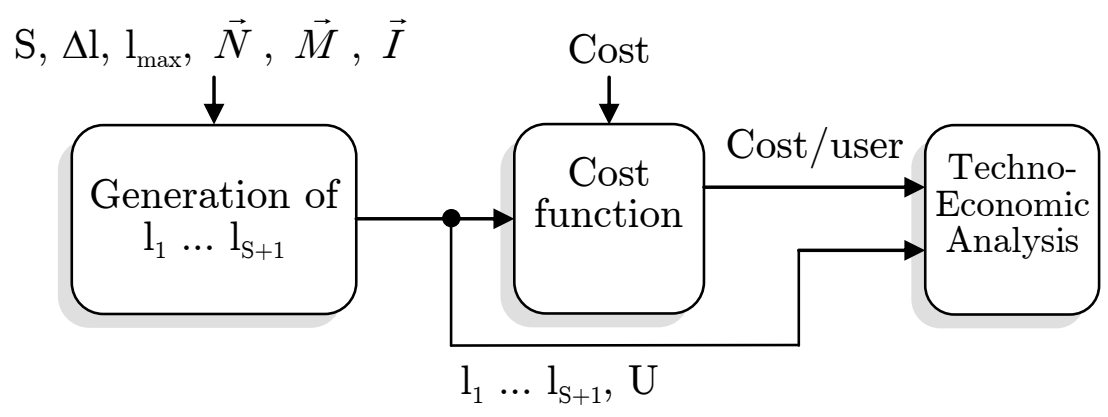

Figure 5.11: Flowchart to identify the optimal component position to minimize the cost.

The method used to find the optimal WR position is illustrated in the following section. The design tool automatically generates all possible combinations of $l_{\mathrm{s}}$ to $l_{\max }$ dependent on $\mathrm{S}, \Delta \mathrm{l}$ and $\mathrm{l}_{\max }$. To verify if our tool works correctly, the number of automatically generated $l_{1} \ldots l_{S+1}$ combinations are controlled. Thus, the number of possibilities acts as a control on the automatically generated distances of the design tool.

When we consider a single-stage WDM PON, the number of possibilities of both distances is $\left(l_{\max } / \Delta \mathrm{l}\right)+1$. An example with $\mathrm{S}=1, \mathrm{l}_{\max }=150$ and $\Delta \mathrm{l}=50$ is shown in Figure 
5.12 , in which the case $\mathrm{S}=1$ is marked. The column labels in Figure 5.12 is considered for the case $\mathrm{S}=4$ and must be adapted for other cases of $\mathrm{S}$. The number of possibilities is obvious with 4 (q.v. column 1 in Figure 5.13). For a two-stage WDM PON, the number of possibilities is $4+3+2+1=10$ and is illustrated in Figure 5.12 for the case $\mathrm{S}=2$. This case is shown in another construction in Figure 5.13 (column $\mathrm{S}=2$ ) in which the number of possibilities is the sum of the possibilities in stage 1 and can be expressed with (little Gauss)

$$
\mathrm{p}(\mathrm{n})=\sum_{\mathrm{i}=1}^{\mathrm{n}} \mathrm{i}=\frac{\mathrm{n}(\mathrm{n}+1)}{2}
$$

whereas $n=\left(l_{\max } / \Delta \mathrm{l}\right)+1$. It is obvious that for an arbitrary stage the number of possibilities is the sum of the number of possibilities of the previous stage and so on (see Figure 5.13).

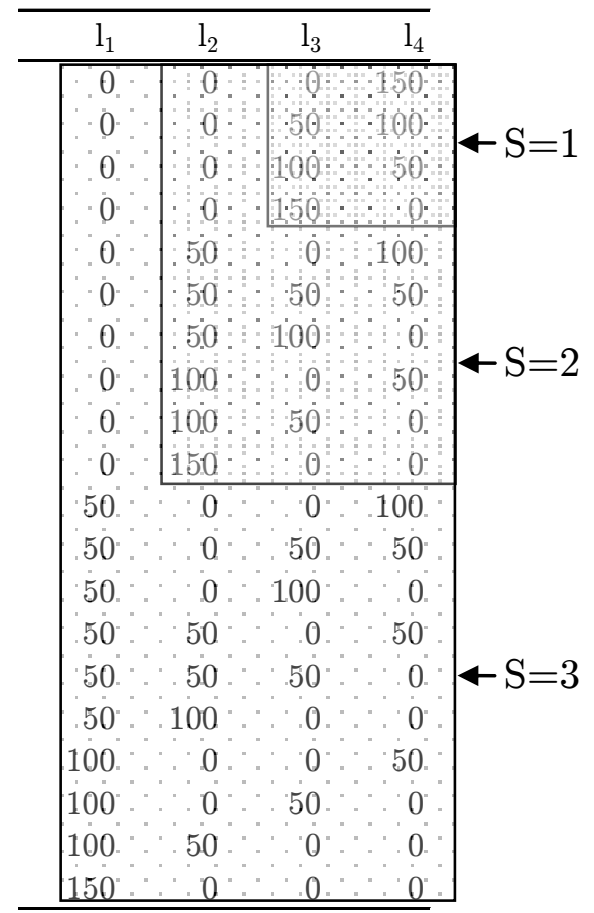

Figure 5.12: Generated link length

by the design tool with

$\mathrm{S}=3, \mathrm{l}_{\max }=150, \Delta \mathrm{l}=50$.

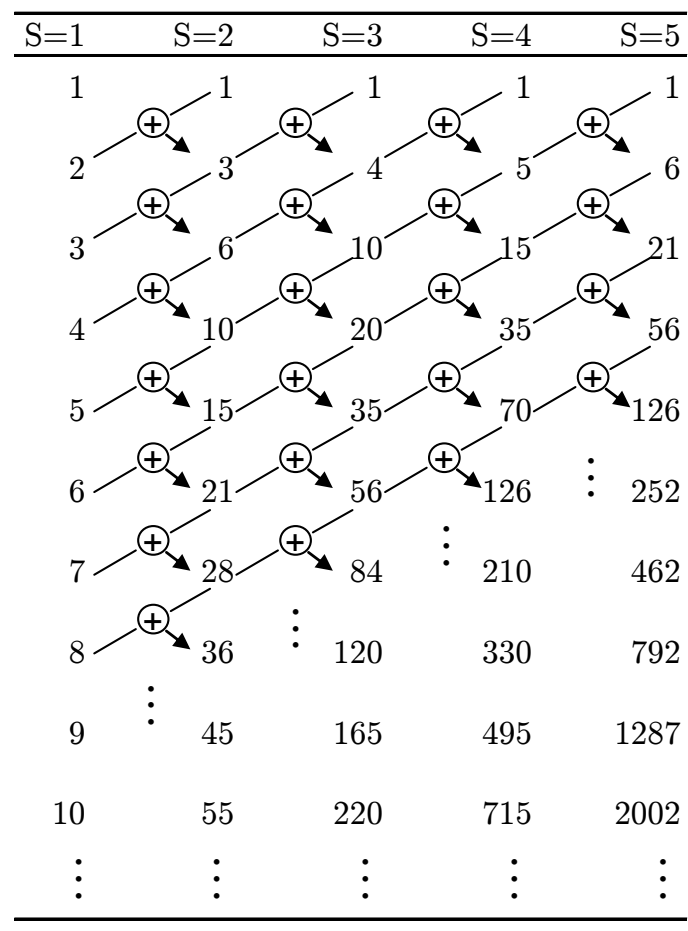

Figure 5.13: Number of possible $1_{\mathrm{s}}$ to $\mathrm{l}_{\max }$ combinations with linearly increased $\left(\mathrm{l}_{\max } / \Delta \mathrm{l}\right)+1$ numbers.

As a result, the number of possibilities in dependence of $n=\left(l_{\max } / \Delta \mathrm{l}\right)+1$ can be generally formulated as follows

$$
\begin{aligned}
& p(n)=\sum_{u=1}^{n} \sum_{v=1}^{n-u+1} \cdots \sum_{\mathrm{z}=1}^{n-y+1} \sum_{i=1}^{n-z+1} \frac{n(n+1)}{2} \\
& \downarrow \downarrow \downarrow \downarrow \downarrow
\end{aligned}
$$

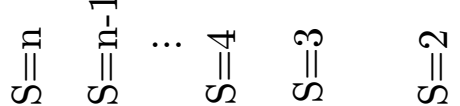


With equation (6.4), the number of generated WR positions helps to verify whether or not our tool works correctly. It can be used to determine or pre-estimate the number of generated WR positions for a given number of $\mathrm{S}, \mathrm{l}_{\max }$ and $\Delta \mathrm{l}$ to reduce the computational complexity. On the basis of the methodologies described before, two examples are calculated for a better understanding of the influencing factors and are explained in the next subsection.

\subsubsection{Performance Analysis}

The first evaluation of the WR position shows the influence of the number of stages on the cost per user. For an equitable comparison among the number of stages, an architecture is chosen in which the vector $\overrightarrow{\mathrm{I}}$ is always 4 . This results in always the same cable with 4 fibers independent of the number of stages. The number of stages varies between 1 and 5 with $\mathrm{U}=8,16,32,64$ and 128 respectively. Further influencing variables are chosen as follows: $l_{\max }=1500 \mathrm{~m}, \Delta \mathrm{l}=50 \mathrm{~m}, \overrightarrow{\mathrm{N}}=[1,2,4,8,16], \overrightarrow{\mathrm{M}}=[8,8,8,8,8]$ and $\overrightarrow{\mathrm{I}}=[4,4,4,4,4]$. In subject to $\mathrm{S}<5$, only the first part of the vectors $\overrightarrow{\mathrm{N}}, \overrightarrow{\mathrm{M}}$ and $\overrightarrow{\mathrm{I}}$ are used.

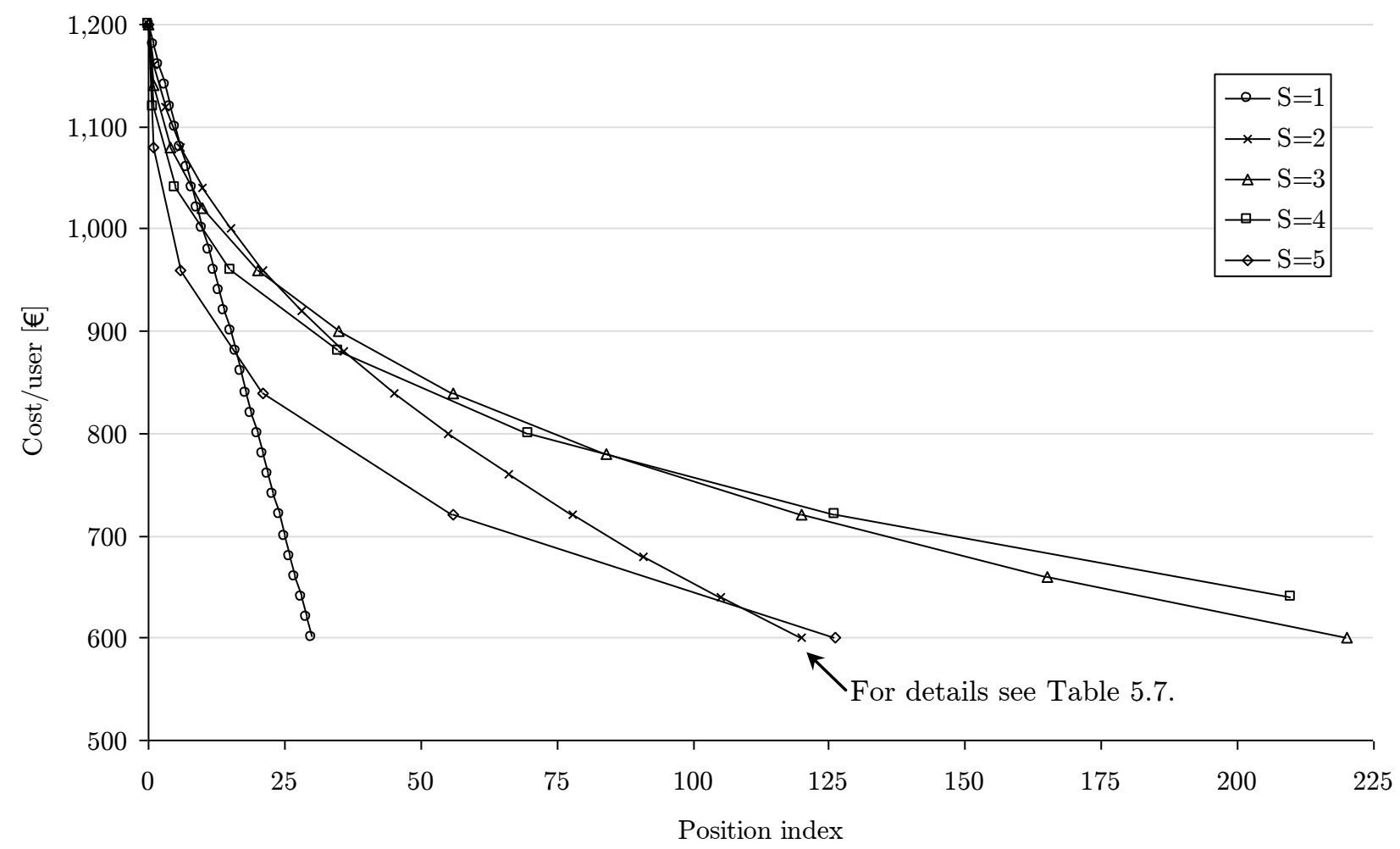

Figure 5.14: Cost per user as a function of the WR position in a multistage WDM PON in dependence of $\mathrm{S}$ with $\mathrm{U}=8,16,32,64,128$ for $\mathrm{S}=1-5$ respectively, $\mathrm{l}_{\max }=1500 \mathrm{~m}, \Delta \mathrm{l}=50 \mathrm{~m}, \quad \overrightarrow{\mathrm{N}}=[1,2,4,8,16]$, $\overrightarrow{\mathrm{M}}=[8,8,8,8,8]$ and $\overrightarrow{\mathrm{I}}=[4,4,4,4,4]$. The vectors $\overrightarrow{\mathrm{N}}, \overrightarrow{\mathrm{M}}$ and $\overrightarrow{\mathrm{I}}$ are shown for $\mathrm{S}=5$. For smaller numbers of S only the first required part of the vectors $\vec{N}, \overrightarrow{\mathrm{M}}$ and $\overrightarrow{\mathrm{I}}$ are used.

For a well arranged illustration, the cost per user are sorted in ascending order for each $\mathrm{S}$ and marked with a position index. WR positions with the same costs are not displayed for clarity. The costs start at its maximum independent of the number of stages at the position index 0 . At this point, all WRs are located at the OLT. This can 
be explained by the space demultiplexing function of a multistage WDM PON in which the fiber count increases stage by stage and is a maximum if all WRs are located at the OLT which results in the highest cost per user.

The minimum of the cost per user can be reached if all WRs are located at the ONU. This case is true independent of the number of stages and the architecture but is not realistic in practice. Our analysis shows us many other WR positions, which also results in a minimalization of costs. For example, the minimum cost for $\mathrm{S}=2$ in Figure 5.14 (arrow marked point) can be reached with many different WR positions. All the WR positions for this minimum cost points are depicted in Table 5.7. In this example, the WR of the second stage is always located at the $\mathrm{ONU}\left(\mathrm{l}_{3}=0\right)$ and the WR in the stage 1 can be moved from the OLT $\left(l_{1}=0, l_{2}=1500\right)$ to the ONUs $\left(l_{1}=1500\right)$ and always leads to a minimum of costs.

In practice, the number of users that must be reached by means of a WDM PON with a deliberate architecture is

\begin{tabular}{rccr}
\hline $\begin{array}{c}\mathrm{l}_{1} \\
{[\mathrm{~m}]}\end{array}$ & $\begin{array}{c}\mathrm{l}_{2} \\
{[\mathrm{~m}]}\end{array}$ & $\begin{array}{c}\mathrm{l}_{3} \\
{[\mathrm{~m}]}\end{array}$ & $\begin{array}{c}\text { Cost/ } \\
\text { user }[€]\end{array}$ \\
\hline 0 & 1500 & 0 & 600 \\
50 & 1450 & 0 & 600 \\
100 & 1400 & 0 & 600 \\
150 & 1350 & 0 & 600 \\
200 & 1300 & 0 & 600 \\
& $\vdots$ & & \\
& & & \\
1400 & 100 & 0 & 600 \\
1450 & 50 & 0 & 600 \\
1500 & 0 & 0 & 600 \\
\hline
\end{tabular}

Table 5.7: Example of minimum cost WR positions with $\mathrm{S}=2$ (q.v. Figure 5.14). often known. Thus, a lot of possible architectures for each number of $\mathrm{S}$ exist to implement a multistage WDM PON with a specific number of users. The question is which architecture and which WR positions must be chosen to implement the network with a minimum of fiber cost for a constant number of users. In this example, the number of users are freely chosen with 64 and with $\mathrm{n}_{\omega}=32, \mathrm{l}_{\max }=1500$ and $\Delta \mathrm{l}=50 \ldots 350$ in dependence of the number of stages to achieve well arranged WR positions. The computation with the design tool results in a lot of possible architectures. For each number of stages, an arbitrary architecture is chosen (see Table 5.8).

\begin{tabular}{llllll}
\hline $\mathrm{S}$ & \multicolumn{1}{c}{$\overrightarrow{\mathrm{M}}$} & \multicolumn{1}{c}{$\overrightarrow{\mathrm{N}}$} & $\overrightarrow{\mathrm{I}}$ & $\mathrm{U}$ & $\mathrm{n}_{\omega}$ \\
\hline 1 & {$[64]$} & {$[1]$} & {$[2]$} & 64 & 32 \\
2 & {$[8,32]$} & {$[1,2]$} & {$[2,4]$} & 64 & 32 \\
3 & {$[4,4,16]$} & {$[1,2,4]$} & {$[2,2,2]$} & 64 & 32 \\
4 & {$[4,4,8,8]$} & {$[1,2,4,8]$} & {$[2,2,2,4]$} & 64 & 32 \\
5 & {$[4,4,4,4,4]$} & {$[1,2,4,8,16]$} & {$[2,2,2,2,2]$} & 64 & 32 \\
\hline
\end{tabular}

Table 5.8: Compared architectures with $\mathrm{U}=64$ and $\mathrm{n}_{\omega}=32$ for each architecture.

The evaluation will show the influence on the cost per user in comparison to the number of stages. For clarity, the costs per user are sorted in ascending order for each $\mathrm{S}$ and marked with a position index, whereas WR positions with the same costs are not displayed. The results are displayed in Figure 5.15.

Similar to the previous example (Figure 5.14), the highest costs occur when each WR is located at the OLT, and costs decline as the distance between the WRs and the ONUs decreases. In each architecture, the minimum costs are reached when all WRs are located at the OLT. This is not realistic in practice, but likewise, as in the previous example, the minimum cost point can be reached with many other WR positions. A detailed examination of a calculated point (see arrow marked point in Figure 5.15) with $\mathrm{S}=5$ will be demonstrated. This point is displayed in detail in Table 5.9 where many minimum cost WR positions are shown. 


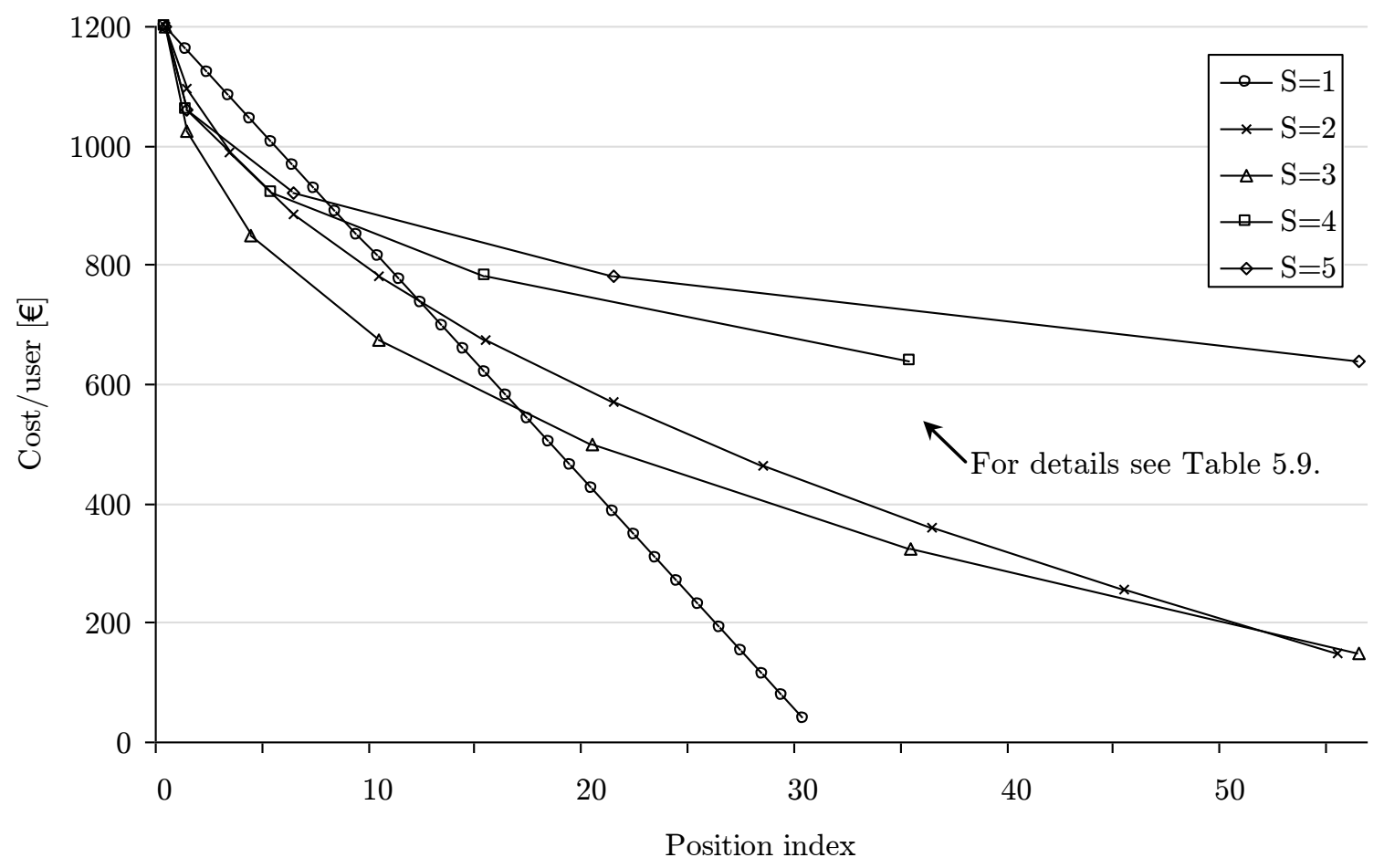

Figure 5.15: Cost per user as a function of the WR position in a multistage WDM PON in dependence of $\mathrm{S}$ with $\mathrm{U}=64, \mathrm{~S}=1-5, \mathrm{l}_{\max }=1500 \mathrm{~m}, \Delta \mathrm{l}=50 \mathrm{~m}-350 \mathrm{~m}$. The simulated vectors $\overrightarrow{\mathrm{N}}, \overrightarrow{\mathrm{M}}$ and $\overrightarrow{\mathrm{I}}$ are shown in Table 5.8.

Another important characteristic can be realized in Figure 5.14 and Figure 5.15. The point at the least possible fiber cost depends on the number of stages, $l_{\max }$ and the selected architecture once again depend on $\mathrm{U}, \mathrm{n}_{\omega}$ and $\mathrm{U}_{\omega}$.

Our evaluation of many architectures show that for increasing values of $\mathrm{S}$ the number of WR positions which results in a cost minimum is also increased. In other words, with a higher number of stages, the network is more flexibel concerning the WR positions to reach a minimum of fiber cost.

\begin{tabular}{rcrrcr}
$\begin{array}{c}\mathrm{l}_{1} \\
{[\mathrm{~m}]}\end{array}$ & $\begin{array}{c}\mathrm{l}_{2} \\
{[\mathrm{~m}]}\end{array}$ & $\begin{array}{c}\mathrm{l}_{3} \\
{[\mathrm{~m}]}\end{array}$ & $\begin{array}{c}\mathrm{l}_{4} \\
{[\mathrm{~m}]}\end{array}$ & $\begin{array}{c}\mathrm{l}_{5} \\
{[\mathrm{~m}]}\end{array}$ & $\begin{array}{c}\text { Cost } / \\
\text { user }[€]\end{array}$ \\
\hline 0 & 0 & 0 & 1400 & 100 & 640 \\
0 & 0 & 350 & 1050 & 100 & 640 \\
0 & 0 & 700 & 700 & 100 & 640 \\
0 & 0 & 1050 & 350 & 100 & 640 \\
0 & 0 & 1400 & 0 & 100 & 640 \\
0 & 350 & 0 & 1050 & 100 & 640 \\
0 & 350 & 350 & 700 & 100 & 640 \\
0 & 350 & 700 & 350 & 100 & 640 \\
0 & 350 & 1050 & 0 & 100 & 640 \\
0 & 700 & 0 & 700 & 100 & 640 \\
0 & 700 & 350 & 350 & 100 & 640 \\
0 & 700 & 700 & 0 & 100 & 640 \\
0 & 1050 & 0 & 350 & 100 & 640 \\
0 & 1050 & 350 & 0 & 100 & 640 \\
0 & 1400 & 0 & 0 & 100 & 640 \\
350 & 0 & 0 & 1050 & 100 & 640 \\
& & $\vdots$ & & & \\
1050 & 350 & 0 & 0 & 100 & 640 \\
1400 & 0 & 0 & 0 & 100 & 640
\end{tabular}

Table 5.9: Example of minimum cost WR positions with $\mathrm{S}=4$ (q. v. Figure $5.15)$. 


\subsubsection{Summary}

To summarize the results of the WR position analysis, we can remark that a centralized position of the WR results in the highest costs but is easier to access and thus leads to a less complex maintenance. Moreover, it improves the flexibility. In contrast, a WR position near the ONUs limits the costs of fiber links but makes them difficult to access which results in a more complex maintenance. It must be stated that in a multistage WDM PON, many WR positions exist to minimize the cost.

\begin{tabular}{llll}
\hline WR positions & Cost & Maintenance & Flexibility/Upgradeability \\
\hline OLT & High & Very easy & Maximize the number of used fibers. \\
In the middle & Middle & Complex & \\
ONU & Low & Complex & Reduce the number of used fibers. \\
\hline
\end{tabular}

Table 5.10: Overview of the techno- economic evaluation of different WR positions in a multistage WDM PON.

Therefore, the right solution to be adopted is different for each specific situation. With our design tool, the best solution can be identified considering the differential costs in maintenance, the foreseen necessity to upgrade the multistage WDM PON, the length of the fiber links and the already installed fibers and ducts. Our design tool can be easily adopted for additional required cost functions.

The size of the network in terms of number of users could be evolved in the course of time. Starting from a network solution with WRs located in the central office and with a small number of stages, the network can be easily maintenanced. Next, the network can be adopted to a higher number of stages and, if required, the WRs can be partially moved towards the ONUs which reduces the fiber costs. The goal is a final scenario with the WR in a peripheral position to minimize the fiber costs.

The next chapter presents the implementation of a multistage WDM PON performed at the Institute of Telematics/Network Engineering at the Carinthia University of Applied Sciences in Klagenfurt, Austria. Potential future applications (e.g. video conferencing in realtime over the IP protocol) have been developed and implemented at the multistage WDM PON to demonstrate future possibilities and the capabilities of those networks. 


\title{
6 Test Environment
}

\author{
A the Institute of Telematics/Network Engineering at the Carinthia University of \\ Applied Sciences in Austria, a PtP demonstration access network was realized. \\ 1 In general, the aim is to demonstrate the added value of optical high-speed \\ access networks by means of potential future applications [Schussmann06, \\ Schussmann08a].
}

The goal was to set-up a novel future-proof high-speed optical access network based on WDM with commercially available components. On the other hand, different highbitrate applications were developed in parallel to demonstrate the requirements of the applications and the correct functionality of the network. The maximum transferable bitrate was chosen with $10 \mathrm{Gbit} / \mathrm{s}$, which allows for the bidirectional transmission of a video conference at the highest HDTV quality uncompressed over IP in realtime and further applications such as ultra-high-speed-data download, telepathology and other applications. In principle, this network should also be able to run every imaginable killer application due to the possibilitity of an easy upgrade of the network in terms of the required bitrate.

We demonstrated state-of-the-art capabilities that serve as a basis for future applications. Each provided service is transmitted simultaneously with other services. Here, two THX-sound systems with a Holo-Pro system (transparent freestanding projection screen, extreme high image resolution) and a large projection screen were installed. We are now in the position to present the enormous amount of transmitted information in a unique quality. In the following subsections, the network and the implemented applications are discussed in detail.

\subsection{Implementation}

Contrary to other cutting-edge scientific papers [Maier00, An04, Bock04, Banerjee05, McGarry06], the focus of this test environment is to provide each ONU with at least one wavelength. In principle, many different architectures and implementation possibilities exist, as analyzed and discussed in chapter 3,4 and 5 in more detail. Due to the limited budget on the one hand, and the possibility to expand the network in terms of number of users and number of wavelengths (and thus to increase the bitrate) on the other hand, a three-stage architecture was chosen.

The architecture of the realized test environment is shown in Figure 6.1. Each of the three stages consists of a multiplexer/demultiplexer. Due to the limited budget, only multiplexer/demultiplexer instead of WR are used. The multiplexer/demultiplexer can 
be considered as a WR in which only one input in the case of a demultiplexer and one output in the case of a multiplexer is used.

Our optical access network is engineered for a limited distance of $50 \mathrm{~km}$. The $50 \mathrm{~km}$ link is marked with "WDM distribution" in Figure 6.1. The network consists of 8 ONUs. The $\mathrm{ONU}_{11}-\mathrm{ONU}_{14}$ and $\mathrm{ONU}_{21}-\mathrm{ONU}_{23}$ (all PCs or servers) are connected to one wavelength over a wavelength converter to provide each ONU with the same wavelength. Thus, each ONU can be connected to standard Gigabit Ethernet (GbE) Interfaces which facilitate the set-up of the test environment. $\mathrm{ONU}_{31}$ is a specially configured layer 3 switch and communicates over a maximum of three wavelengths, which is used for the video conferencing tool (see subsection 6.2.2).

The optical line termination OLT is located on the other side of the WDM distribution network. The network structure of the OLT is the same as in Figure 6.1 but structured in a mirror-inverted manner. Thus, the OLT is not depicted in Figure 6.1 for clarity.

In order to support such a pay-as-you-grow concept, the WDM system must be modular. Each WDM channel was realized by using a single Gigabit-Ethernet InterfaceCard or a small form-factor pluggable (SFP) module.

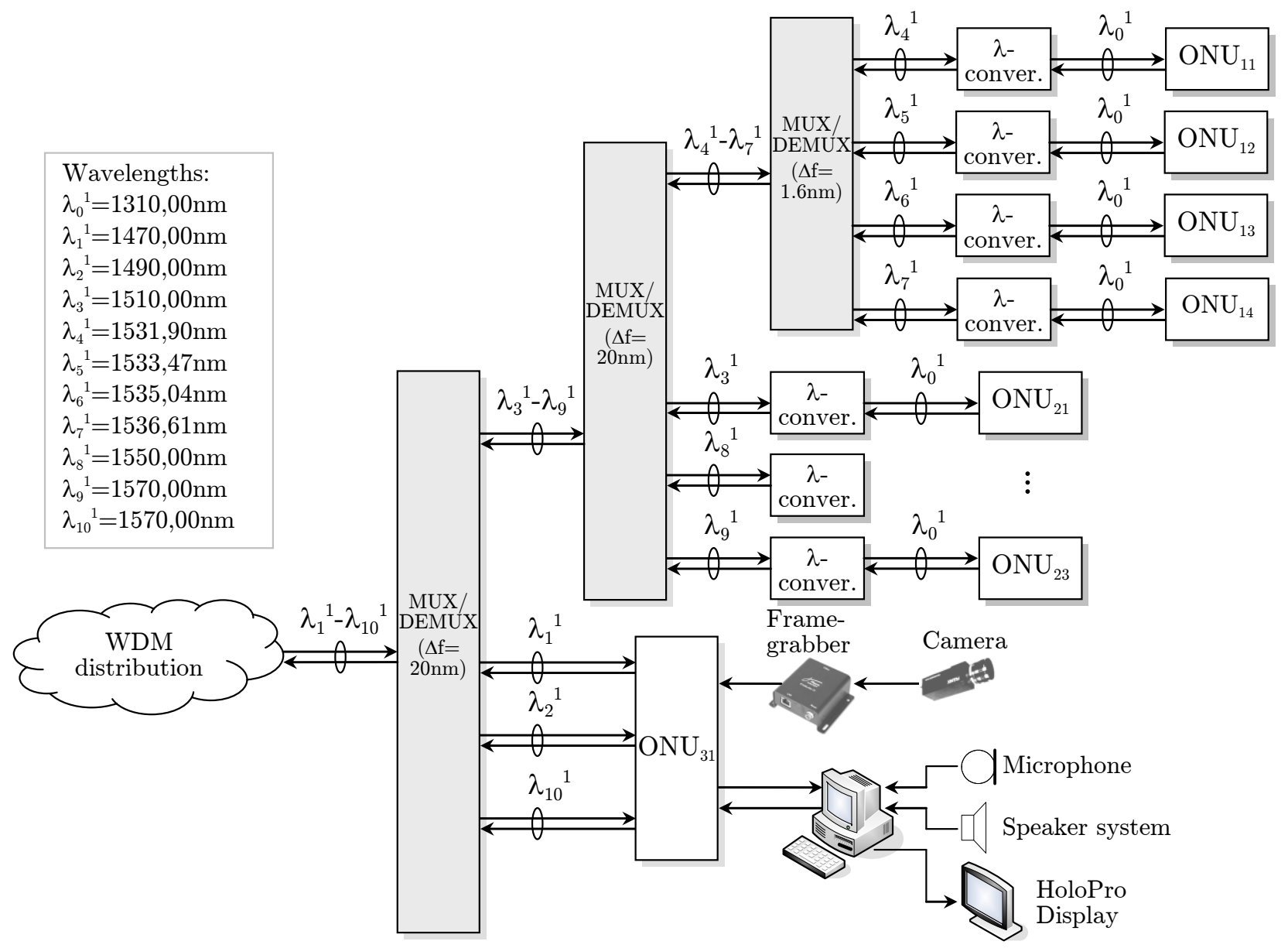

Figure 6.1: An overview of the test environment of the multistage WDM PON at the Institute of Telematics / Network Engineering at the Carinthia University of Applied Sciences in Klagenfurt, Austria. The test environment consists of a three-stage architecture that is linked via a $50 \mathrm{~km}$ monomode fiber (WDM distribution). $\mathrm{ONU}_{11}-\mathrm{ONU}_{14}$ and $\mathrm{ONU}_{21}-\mathrm{ONU}_{23}$ are standard $\mathrm{PCs}$ or speed optimized servers allowing sophisticated high-speed applications. $\mathrm{ONU}_{31}$ is explained in more detail in subsection 6.2.1. 
With our hardware equipment a maximum of 6 CWDM (20nm channel spacing) and a maximum of 25 DWDM (0.8nm channel spacing) channels can be used. At the moment, 6 CWDM and 4 DWDM channels are in use. The wavelength covers a wavelength range between $1470 \mathrm{~nm}$ and $1590 \mathrm{~nm}$ and is shown in a connectivity map in Figure 6.2 [Schussmann06].

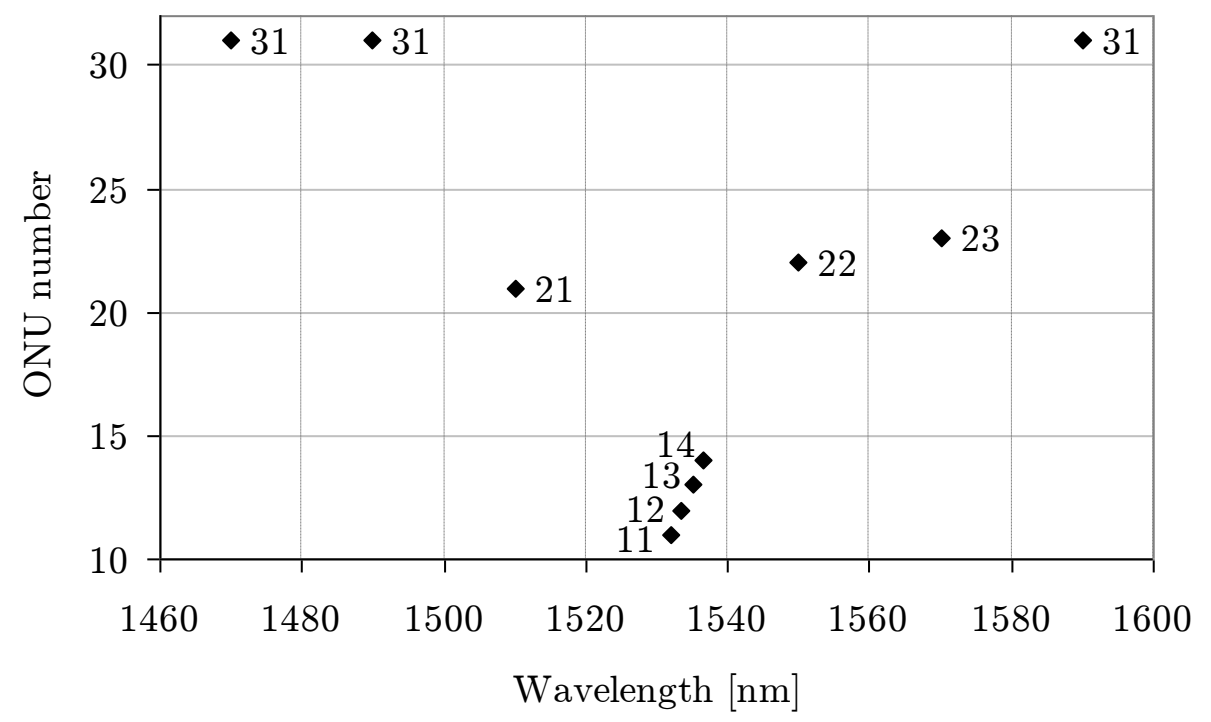

Figure 6.2: Connectivity map for the test environment. 6 CWDM and 4 DWDM channels are used to connect 8 ONUs.

To demonstrate the pay-as-you-grow strategy, the CWDM channel with the wavelength $1530 \mathrm{~nm}$ is replaced by the wavelengths $1531.9 \mathrm{~nm}, 1533.47 \mathrm{~nm}, 1535.04 \mathrm{~nm}$ and $1536.61 \mathrm{~nm}$ which correspond to $\mathrm{ONU}_{11}-\mathrm{ONU}_{14}$ in Figure 6.2. For each replaced CWDM channel a maximum of $20 \mathrm{~nm} / 0.8 \mathrm{~nm}=25$ DWDM channels can be transferred. As a result, if more than 10 optical channels were necessary, we can easily introduce further channels by replacing CWDM with DWDM channels without modifying the WDM PON.

Another interesting question is what the optimal parameter configuration for the test environment is? The aim is to find an optimal parameter configuration to reduce costs and guarantee the correct transmission. Essential physical parameters are simulated like the power budget, chromatic dispersion, waveguide dispersion, material dispersion and the resultant pulse broadening, which is responsible for the limited bitrate [Auner05].

The most important simulation result is shown in Figure 6.3 for three different parameter variations, which are illustrated in Table 6.1. All three systems correspond to commercially available transmitter and receiver components.

\begin{tabular}{lcccr}
\hline & $\tau_{\text {tr }}[\mathrm{ps}]$ & $\tau_{\text {rec }}[\mathrm{ps}]$ & $\Delta \lambda_{\text {tr }}[\mathrm{nm}]$ & Cost [€] \\
\hline System 1 & 50 & 150 & 0.1 & 1,700 \\
System 2 & 100 & 300 & 0.5 & 1,150 \\
\hline System 3 & 150 & 500 & 0.7 & 300 \\
\hline
\end{tabular}

Table 6.1: Parameter variations used in the simulation of the test environment for three different commercially available transceivers. $\tau_{\text {tr }}$ is the rise time of the transmitter, $\tau_{\text {rec }}$ the rise time of the receiver and $\Delta \lambda_{\text {tr }}$ is the spectral broadness of the transmitter. The reported costs were covered by a manufacturer in 2006 . 
Based on the simulation results, it can be deduced that the choice of the proper fiber optic cable is not essential for dimensioning an optical access network. In fact, standard monomode fiber optic cable can be used. However, well selected laser and receiver diodes or rather transmitters allow for transmission rates of up to several Gbit/s per channel over standard monomode fibers (Figure 6.3). In other words, the costs can be significantly reduced by well chosen transmitters.

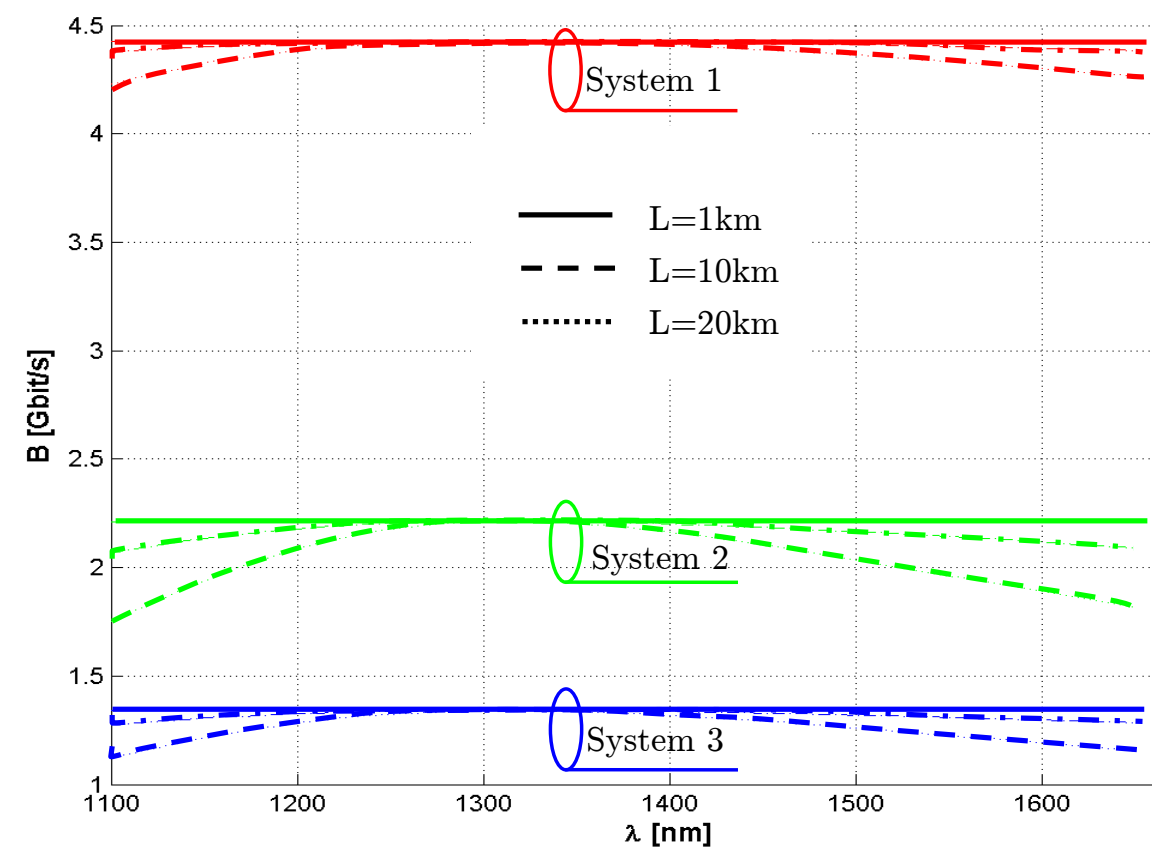

Figure 6.3: Maximum achievable channel bitrate $\mathrm{B}_{0}$ in dependence on the wavelength for three different systems [Auner05]. The used transmitter and receiver parameters are shown in Table 6.1.

In the next section, the developed applications to demonstrate the huge potential of optical access networks which are based on the WDM technique is shown.

\subsection{Applications}

Communication as a social form of interaction, as a way to exchange information, has been influencing life on earth for millions of years. However, over the years, the methods of how to communicate have changed totally.

As the connections get faster and faster, the question is what the benefits are and how the higher bitrates can be used to realize new areas of application. Ultra-high- speed download, video on demand and video conferencing are considered to be killer applications, even for optical networks. In most cases, the scenario of viewing a DVD movie stream from a network server is unthinkable for today's home users. However, a foresight lets us imagine the day when the dream of WDM PONs will come true, when every household will possibly be provided with its own fiber.

Assuming the availability of such a network, high quality video conferencing is of great importance for a modern means of communication as it virtually connects remote spaces as if they were a stone's throw away from each other.

On the basis of the state-of-the-art test environment, this section highlights possibilities for the virtual conferences of tomorrow. It must be mentioned that many other 
applications such as telepathology, video on demand (Vod) and ultra-high-speed download can be presented.

\subsubsection{Video Conferencing}

Video conferencing can be regarded as a real-time bidirectional video streaming process. In contrast, the term "video streaming" basically means a one-way end-to-end transmission of video data via a network. Received data is immediately sent to display. In case of using a buffering method, the data is slightly delayed. The source, for instance, could be a video file from a hard disk, a digital versatile disc (DVD) or a camera.

In a video conferencing environment, only video cameras are used as sources. In most cases it implicates that an audio connection is also established so that both sides can hear and see each other.

The phrase real-time is a sensitive expression und must be precisely defined. Real-time, according to [Klinger03], is defined as follows: "real-time simply means that every operation has to be completed within acceptable delay times, whenever the system ensures completion." Another definition is given in Figure 6.4.

In the case of a video conference, the sample time can be considered as the time between two sampled images. Thus, the video conferencing system works in real-time if a captured image can be transferred and displayed by the receiver within the sample time or, in other words, if the waiting time is greater than or equals zero.

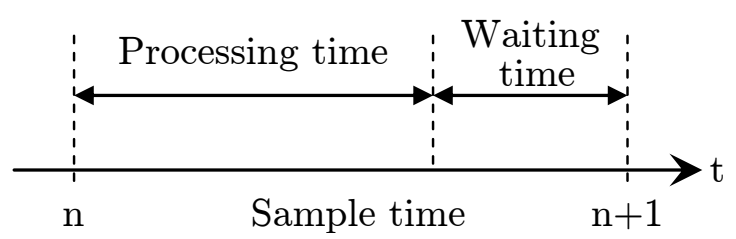

Figure 6.4: Definition of real-time: given a certain sample time, the application works in real-time when the waiting time is greater than or equals zero.

Therefore, we consider our system to run real-time when the processing time between two samples (is equal to two images) is shorter than the sampling period.

\subsubsection{HQHS Streaming}

The video conferencing test environment in its current state is shown in Figure 6.5 (part of the multistage WDM PON in Figure 6.1). Two CWDM multiplexer/demultiplexer systems are connected via an optical fiber with the length of 50 kilometers. On each side, a layer 3 switch is connected via small form-factor pluggable (SFP) devices to the multiplexer. Multimedia PCs and frame grabbers are connected via Gigabit Ethernet to the switches. Frame grabbers and cameras are connected via camera link cables. Concerning the PCs, the audio outputs are connected to a theatrical extensions (THX) sound system and the video outputs are connected to beamers. 


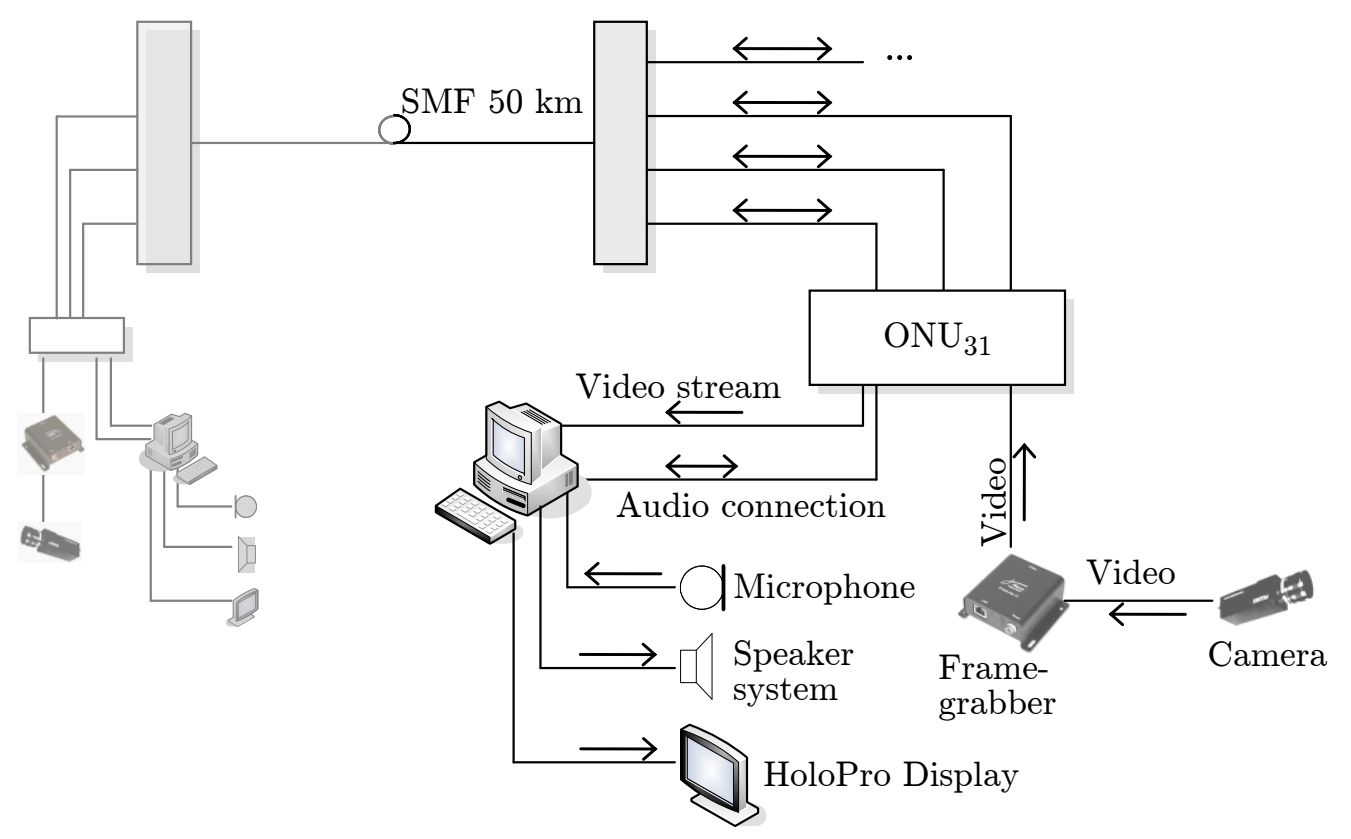

Figure 6.5: Video conferencing in the test environment. It illustrates the video conferencing connections in the current configuration. The frame-grabber captures video data from the camera link and transmits it through the $\mathrm{ONU}_{31}$ (layer 3 switch) on to the optical part of the network. A dedicated GbE connection for each direction is used to transmit the video stream. Another GbE connection is used for transmitting and receiving the audio stream.

In conjunction with video streaming, high-quality high-speed (HQHS) means a maximum of quality concerning the video itself at a minimum of latency. Latency stands for the time it takes to transmit an image from the sender to the receiver. As high video quality is mostly referred to as using an increased resolution, the goal in HQHS streaming is to maximize it. What is more, how a video stream impacts the viewer depends on the frame rate the video is displayed at. So it is necessary to display it at a minimum of 25 frames per second, 30 frames per second at best.

The idea of HQHS streaming indicates that a compression algorithm need not be used as it takes too much time and downgrades quality. Therefore, a fast method for transmitting huge amounts of raw data has to be found. We were urged to develop a fast method for transmitting huge amounts of raw data by using the Windows $\mathrm{XP}^{\circledR}$ operating system.

A video conference offers only half the fun without an audio connection. HQHS audio is a must-have whereas compression should not to be used. As a result of choosing the approach depicted in Figure 6.5, we are able to guarantee an acceptable quality level of Compact Disc Digital Audio (CDDA).

Many different video conferencing tools as well as video streaming applications are available. They were promoted under various licences and with many different features. But they are generally designed to have low bitrate consumptions, so it is possible to use them on slow connections, too. Today, most internet connections do not provide the user with a high speed link; the design of the video conferencing tool is thus comprehensible. In fact, there is no video conferencing tool available that fits the needs of HQHS. They all lack either high resolution or low latency due to the storage of video sequences in all tested video conferencing tools. During this work, a variety of tools have 
been tested (video conferencing tool [Vic07] from the University of California, Berkeley or the VideoLAN [Vid07]) [Angermann05].

The results of the evaluation process confirmed the inexistence of a tool that is capable of HQHS streaming. A modification of existing software seemed to be a far more complex task as no tool provided an architecture that could be extended easily to support uncompressed video data transmission. The advantages of developing a brand new application are the clean structure for the special purpose it can be designed with. Another benefit is provided by the fact that there are no unwanted features which could possibly decrease the overall performance.

In summary, HQHS streaming enables to transmit an uncompressed high quality video stream. In this project we minimized the latency, and chose a resolution of $1600 \times 1200$ pixel by using a bit depth of 10 bit per pixel and a frame rate of $30 \mathrm{fps}$. For an uncompressed high-quality audio stream, we sample with a rate of $44.1 \mathrm{kHz}$ and use a resolution of $16 \mathrm{bit}$. These quality parameters require data rates of approximately $600 \mathrm{Mbit} / \mathrm{s}$ payload to transmit video and audio in an unprecedented quality.

At the moment ultra-elaborate applications show uncompressed video conferencing with a data rate of $6 \mathrm{Gbit} / \mathrm{s}$ using a pixel matrix of 4096 x 2048 pixel [Doug Ramsey et al., http://ucsdnews.ucsd.edu/newsrel/science/iGrid4K.asp, University of California, 2005]. Although we rate this highbrow application not to be economically relevant for the near future, we can adapt our system to those requirements.

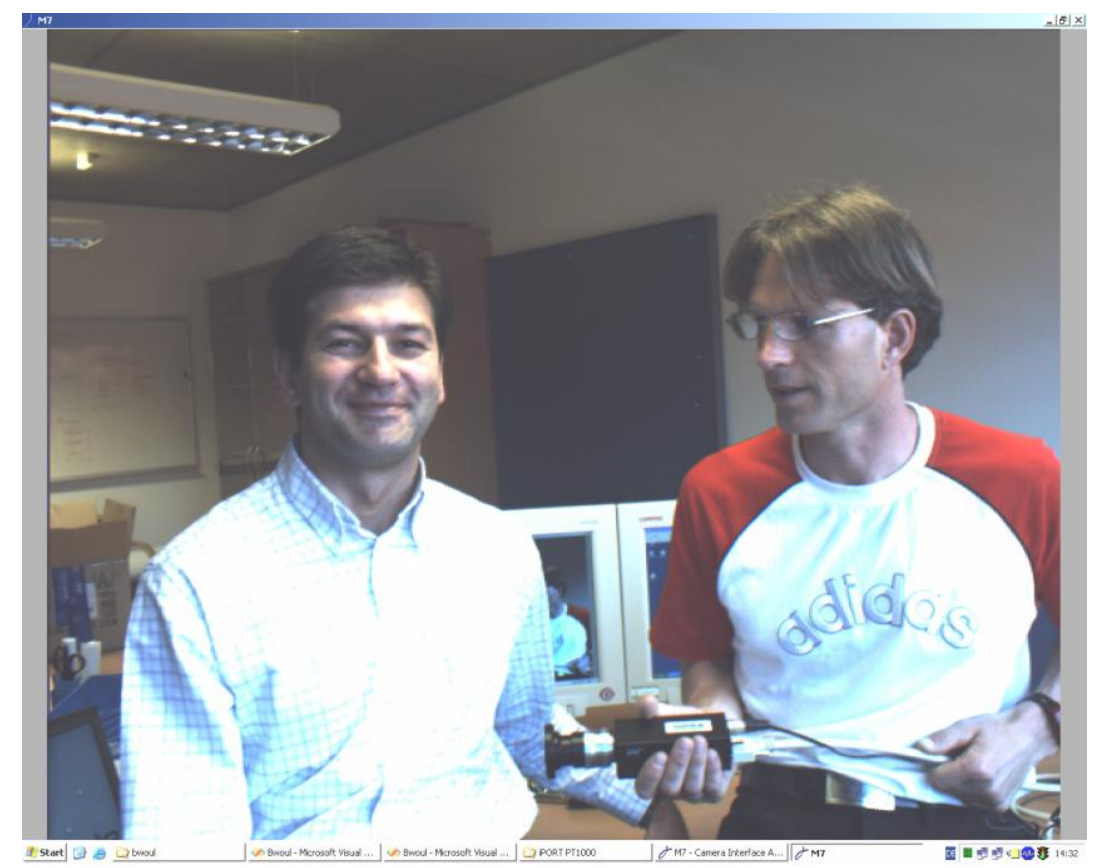

Figure 6.6: Snapshot from the developed video conferencing tool Bwoul (broadcast without usual limits).

Windows Multimedia SDK is used for both capturing audio data from the input device as well as for playing audio data on the output device. The buffer sizes are especially important. The more bytes it takes to fill a buffer, the more latency occurs when streaming. This can be applied to both the sending (capturing) side and the receiving (playing) side. The feat is to find the right value so that the minimum possible latency 
occurs but the audio stream is not interrupted because of too fast buffer switching from one to another.

During the creation of the video conferencing tool, audio streaming was only tested with CDDA quality settings. That is a sample rate of $44.1 \mathrm{kHz}$ with 16 bit samples in stereo. The reason is easily explained: The requirements of HQHS streaming guarantee high quality audio. Most of the sound cards in standard PCs have built-in support for audio input at CDDA quality. So the format was fixed to these settings to be able to do optimizations for it.

A calculation shows the actual payload transferred. As two different cameras are used in the current configuration, the payload is not equal for both sides. It can be calculated using the following formula:

$$
\text { Payload }_{\text {video }}=\mathrm{x}_{\text {res }} \cdot \mathrm{y}_{\text {res }} \cdot \text { bitdepth } \cdot \operatorname{colchan} \cdot \mathrm{fps} \text {. }
$$

The payload in bits per second is a multiplication of the horizontal resolution $\left(\mathrm{x}_{\mathrm{res}}\right)$, the vertical resolution $\left(\mathrm{y}_{\text {res }}\right)$, the bit depth, the number of color channels (colchan) and the frames per second (fps). Both sides are equipped with a Jai CV M8-CL camera, which has a resolution of $1600 \times 1200$ pixels at a bit depth of 10 bit per pixel. When streaming at the highest possible frame rate, which is 30fps, this results in the following payload

$$
\text { Payload }_{\text {video }}=1600 \cdot 1200 \cdot 10 \cdot 1 \cdot 30=576 \mathrm{Mbit} / \mathrm{s} \text {. }
$$

As far as audio is concerned, the payload can be calculated using the formula

$$
\text { Payload }_{\text {audio }}=\mathrm{sps} \cdot \mathrm{bps} \cdot \mathrm{chan}
$$

where sps is the number of samples per second, bps is the number of bits used per sample and chan is the number of channels used. The Compact Disc Digital Audio (CDDA) format, which is used for the video conferencing, has a sampling rate of $44.1 \mathrm{kHz}$ per second and a 16bit sampling. Two channels are used. The consumption in a transmission is

$$
\text { Payload }_{\text {audio }}=44100 \cdot 16 \cdot 2=1,4112 \mathrm{Mbit} / \mathrm{s} \text {. }
$$

As can be seen in the equation above, audio data is negligible compared to video data in terms of bitrate consumption. The accumulated payload consists of the video data from the Jai CV M8-CL camera and the CDDA quality audio data

$$
\text { Payload }_{\text {total }}=\text { Payload }_{\text {video }}+\text { Payload }_{\text {audio }}=577.4112 \mathrm{Mbit} / \mathrm{s} \text {. }
$$

It is important to state that the above calculation shows only the actual data that is then displayed (video) and played (audio), not the overhead consumed by the transmission protocols. 


\section{Problems and Issues}

A video/audio conference has to deal with physical influences, too. For example, two separated rooms where the conference is to take place, pose the problem of how audio data should be recorded and played simultaneously without getting feedback loops. Of course, every participant could use a headset for the communication, but that is not always convenient because it is a restriction.

So the intention is to play audio on a speaker system and set up one or more microphones in this room. Depending on the type of microphone, its characteristics and the volume of the sound played on the speaker system, it is basically possible to establish a conference without echo. This is the general approach that has been chosen for all the tests with the developed video conferencing tool. It is a compromise but it works well when using cardioid dynamic microphones.

However, the speaker has to be very close to the microphone (at least 40 centimeters). For testing purposes, this is an acceptable situation but could be too restrictive in a business meeting. 


\section{Conclusion and Perspective}

7 he already enormous and ever increasing bandwidth demand justifies the need for high-speed access networks. In particular, WDM technology is commonly viewed as the preferred choice in building such future high-speed access networks. In WDM networks, multiple wavelength channels operating at electronic transmission rates are supported in parallel to fully utilize the enormous potential bandwidth of a single optical fiber. Furthermore, WDM PONs appear to be the preferred architecture for the development of highly flexible future access networks.

To make sure that multistage WDM PONs can be considered as viable, promising alternatives to current access network developments such as WLAN and xDSL, several requirements have to be fulfilled. First, they have to be realizable and cost-effective. Specifically commercially available low cost WRs, lasers and receivers are of high interest in this context. Second, they should provide scalability in terms of the number of wavelengths per user and in terms of the number of users. Third, they have to support an independent multi-provider solution and an independent multi-service solution which results in an uncoupling between the network and the service provider. It must be stated that no real deployment currently exist for multistage WDM PONs.

The first part of this dissertation thesis provides an overview of optical access networks and especially of passive optical networks (PONs). The taxonomy is based on different multiplexing schemes like space, time and wavelengths. An introduction of necessary components to realize those networks was also delineated. In this context, state-of-theart standardizations were discussed.

The paradigm shift of at least one wavelength per user and service is the basis for further considerations. It is a long-term and flexible alternative to the TDM PON currently being deployed by operators to support the extraordinary data traffic growth in access networks.

This work has focused on the design and analysis of single- and multistage WDM PONs with increased or decreased coarseness based on a passive tree topology (chapter 3 and 4). The optical key component is the static wavelength router (WR). With the described methodology, single- and multistage WDM PONs can be designed and calculated with the objective to fulfill the introduced paradigm shift in section 2.5.

New methods based on a generalization of the WTM in combination with the introduction of a new matrix operator (based on a modified matrix multiplication) 
enable the calculation of a WR independent from the size, coarseness factor and the number of used FSRs in one step. As a consequence, the design of a single-stage WDM PON and the adherence of the paradigm shift in section 2.5 are feasible.

In multistage WDM PONs, new design methods allow the efficient calculation recursively for IC and DC WDM PONs. These new methods enable the calculation of the entire nework independent from the size of the network in terms of the number of users and the number of wavelengths used.

In the course of this work, a design tool which is based on the proposed design rules has been developed to design and calculate such multistage WDM PONs. This tool helps to better understand such networks and allows to study and to introduce new architectures. Due to technology constraints the capacity limits and the maximum number of users are shown for single- and multistage WDM PONs. In order to make sure that multistage WDM PONs work properly with respect to the power budget, it could be demonstrated that these kinds of networks work well up to 4 stages.

Furthermore, our design tool is able to calculate the connectivity map. It serves to comprehend which source (wavelength) at the OLT is connected to which ONU. The connectivity map can be calculated from the OLT in the direction of the ONUs or vice versa. Commonly the connectivity map is determined by starting to define the wavelengths at the OLT, and then the calculation is done in the direction of the ONUs. In this case, the calculation is based on 3D matrices with standard matrix multiplications. Furthermore, the calculation can be done in the other direction if the wavelengths are assigned at the ONUs. The calculation, starting at the ONUs in the direction of the OLT, can also be done with inverse 3D matrices and inverse matrix multiplication operators.

During this work, another novel method to calculate such a multistage WDM PON has been demonstrated. This was done with a generalization of the WTM combined with a modified matrix multiplication (section 3.3).

Further on, techno-economic evaluations of single and multistage WDM PONs are accomplished with our developed design tool. Architectures minimizing the costs are identified. The selection depends on crucial requirements such as the number of users and the number of wavelengths per user. Another important issue in minimizing the cost is the position of the WR. Those WR positions that minimize the costs can be identified for any architecture. I was able to show that for any architecture there are many WR positions that minimize the cost. Thus, the most applicable minimum cost architecture can be chosen.

At the institute of Telematics/Network Engineering at the Carinthia University of Applied Sciences in Austria, a multistage WDM PON test environment was realized. The test environment is performed with 10 optical channels, each one with a data rate of $1 \mathrm{Gbit} / \mathrm{s}$ over a distance of $50 \mathrm{~km}$. With our testbed we are able to demonstrate most highly sophisticated communication applications such as ultra fast data transfer, videoon-demand (VoD), video-conferencing in real-time over IP, telepathology and so on. The testbed can be easily upgraded in terms of the number of wavelengths and the number 
of users. Now we are one step closer to uniting all existing communication services that we denote as reconvergence of communication services (RoCS) [Schussmann08a].

The results obtained in this dissertation thesis provide inputs for further investigations. Further research activities should support the usage of other optical components to obtain a greater flexibility and thus more possibilities to design an optical network that is based on the WDM technique. To obtain further options, the switching of wavelengths could be an issue for further research activities. Thanks to the non-blocking behavior of the well-known optical cross connects (OXC); they allow for switching wavelengths from an arbitrary input port to any output port. Further research activities should examine optical multistage WDM PONs consisting of both, static WR and OXC.

This work is based on the equidistant wavelength spacings in the discrete wavelength domain. Further research should use a non-equidistant discrete wavelength grid. The outcome of the non-equidistant wavelength grid is the feasibility of using different wavelength spacings. It should moreover increase the expandibility in regard to the number of wavelengths and number of users. It also increases the flexibility and allows for a pay-as-you-grow strategy. Starting with broad wavelengths spacings and therefore generating lower costs, the network can be upgraded in terms of the number of wavelengths with smaller wavelengths spacings.

In a multistage WDM PON, different coarseness factors are unfavorable situations and do not help to avoid installation difficulties, maintenance and inventory. The question is: is it possible to design a multistage WDM PON with a constant coarseness factor for given requirements which fulfill the paradigm shift? Therefore, further research must be made to study multistage WDM PONs with a constant coarseness factor.

Currently, the researched architectures have been restricted to tree topologies only. However, bus topologies are more fail-safe than tree topologies (q.v. self-healing in SDH) and thus a topic in optical access networks.

A constraint in the design process is the same number of wavelengths per user for all users. In a real deployment, this assumption limits the employment of multistage WDM PONs. As a result, the design rules in chapter 4 must be adjusted. Another limited constraint is the same coarseness factor and the same size of the WR in stage s. Without these limitations, multistage WDM PONs can be more flexible. This means that the number of possible network architectures increases significantly.

In case of using more than one wavelength per user, standards are needed to define which application is assigned to which wavelength. This means that a protocol must be developed to offer the exchange of the control information needed. Moreover, an intelligent scheduler is needed in the OLT to fairly assign the limited number of wavelengths to users and applications. This is an important topic and has the potential for future research activities. 


\section{Bibliography}

[Agrawal02] P. Agrawal. Fiber-Optic Communication Systems. WileyIntersience, 2002, ISBN 0-471-21571-6.

[Ahn02] B. Ahn, Y. Park. A Symmetric-Structure CDMA-PON System and Its Implementation. IEEE Photonics Technology Letters vol.14, 9. September 2002, pp. 1381-1383.

[An04] F. An, K.S. Kim, D. Gutierrez, S. Yam, E. Hu, K. Shrikhande, L.G. Kazovsky. SUCCESS: A Next-Generation Hybrid WDM/TDM Optical Access Network Architecture. Journal of Ligthwave Technology, vol.22, no.11, November 2004, pp. 2557-2569.

[Andersen97] N.E. Andersen et al. Broadbandloop: A Full-Service Access Network for Residential and Small Business Users. IEEE Communications Magazine, December 1997, pp. 88-93.

[Angermann05] R.G.C. Angermann. Video Conferencing in High Bandwidth Access Networks. Diploma thesis, University of Applied Sciences, School of Telematics / Network Engineering, Klagenfurt, Austria, 24.06.2005.

[Aoyama04] T. Aoyama. Development of Broadband Applications based on Super-High Definition Image. ECOC 2004 Proceedings, vol. 5, pp. $18-21$.

[Asimov62] I. Asimov. Magazine of Fantasy and Science Fiction science column. August 1962.

[Atsushi04] M. Atsushi, H. Oomori. T. Takagi. Extremely Compact DFB Laser Modules for the DWDM and CWDM Systems in the Wide Temperature Range. ECOC 2004 Proceedings, Vol.4, pp. 866-867.

[Auner05] G. Auner. Anforderungsanalyse und Implementierung optischer xWDM-Zugangsnetze. Diplomarbeit, Fachhochschule Telematik / Netzwerktechnik, Klagenfurt, Austria, 24.06.2005. 
[Bachhiesl03] P. Bachhiesl, M. Prossegger, G. Paulus, J. Werner, H. Stögner. Simulation and Optimization of the Implementation Costs for the Last Mile of Fiber Optic Networks. Networks and Spatial Economics, 3, 2003, pp. 467-482.

[Bachhiesl04] P. Bachhiesl, M. Prossegger, H. Stögner, J. Werner, G. Paulus. Cost Optimal Implementation of Fiber Optic Networks in the Access Net Domain. Proceedings CCCT04 - Int. Conf. on Computing, Communications and Control Technologies, Austin, USA, Vol III, 2004, pp. 344-349.

[Banerjee05] A. Banerjee, Y. Park, F. Clarke, H. Song, S. Yang, G. Kramer, K. Kim, B. Mukherjee. Wavelength-division-multiplexed passive optical network (WDM-PON) technologies for broadband access: a review [Invited]. Journal of Optical Networking, vol.4, no.11, November 2005, pp. 737-758.

[Barry93] R.A. Barry, P.A.Humblet. Latin Routers, Design and Implementation. Journal of Lightwave Technology, vol.11, no.5/6, May/June 1993, pp. 891-899.

[Bell96] P. Bell, R. Walling, J. Peacok. Costing the access network - an overview. BT Technology, vol.14, no.2, April 1996, pp. 128-132.

[Bell98]

[Bengi02]

[Biraghi02]

P. Bell, P. Trigger. Access network life-cycle costs. BT Technolology, vol.16, no.4, October 1998, pp. 165-174.

K. Bengi. Optical Packet Access Protocols for WDM Networks. Kluwer Academic Publishers 2002, ISBN 140207042X.

A.M. Biraghi, K. Cobb, S.C. Cantone, L. Greborio, R. Mercinelli, R. Walling. Survey of future broadband services. Ist for Broadband Europe, October 9, 2002.

[Birolini97] A. Birolini. Reliability Engineering, Theory and Practice. Springer Berlin, $4^{\text {th }}$ edition, 1997, ISBN 3-540-66385-0.

[Bludau04]

[BOBAN00]

W. Bludau. Lichtwellenleiter in Sensorik und optischer Nachrichtentechnik. Springer Verlag, 2004, ISBN 3-540-63848-2.

Project from EURESCOM. Building and Operating Broadband Access Networks. DWDM Technologies for Access Networks. http://www.eurescom.de/ pub-deliverables/p900-

series/p917/d10Vol1/p917d10vol1.pdf, [last access 21.11.2006]. 
[Bock04]

[Borella98]

[Born05]

[Brackett90]

[Bradley99]

[Brealey06]

[Cahn98]

[Caviglia98]

[Chang03]

[Chen99]

[Coffman01]

[DeCusatis02]

[DeCusatis06]
C. Bock, J. Prat, J. Segarra, G. Junyent, A. Amrani. Scalable Twostage Multi-FSR WDM-PON Access Network Offering Centralized Dynamic Bandwidth Allocation. ECOC 2004 Proceedings, vol. 2, pp. 278-279.

A. Borella, G. Cancellieri, F. Chiaraluce. Wavelength Division Multiple Access Optical Networks. Artech House, 1998, ISBN 0890066574 .

M. Born, E. Wolf. Principles of Optics. Cambridge University Press, $7^{\text {th }}$ edition, 2005, ISBN 0-521-642221.

C.A. Brackett. Dense Wavelength Division Multiplexing Networks: Principles and Applications. IEEE Journal on Selected Areas in Communications, vol.8, no.6, Aug. 1990, pp. 948-964.

P.J. Bradley, N. Caponio, M. Calzavara, M. Cerisola, G. Destefanis, P. Pastorino. Applications of WDM technologies for PON access network upgrading. CSELT Technical Report, 1999, pp. 669-676.

R.A. Brealey, S.C. Myers, F. Allen. Corporate Finance. McGrawHill Education, $8^{\text {th }}$ edition, 2006, ISBN 0-07-111551-X.

R.S. Cahn. Wide Area Network Design. Academic Press, 1998, ISBN 1-55860-458-8.

F. Caviglia, A. Gnazzo, G. Baroncelli. Splitter position in a PON based architecture: a techno-economic analysis. SPIE vol.3408, May 1998, pp. 40-46.

K. Chang. Handbook of Optical Components and Engineering. John Wiley \& Sons, 2003, ISBN 0-471-39055-0.

W.Y. Chen. The Development and Standardization of Asymmetrical Digital Subscriber Line. IEEE Communications Magazine, May 1999, pp. 68-72.

K.G. Coffman, A.M. Odlyzko. Internet growth: Is there a "Moore's law" for data traffic?. AT\&T Labs - Research, Revised Version, June 4, 2001.

C. DeCusatis. Handbook of Fiber Optic Data Communication. Academic Press, 2002, ISBN 0-12-207891-8.

C.M. DeCusatis, J.S. DeCusatis. Fiber Optic Essentials. Academic Press, 2006, ISBN 0-12-208431-4. 
[Dixit03]

[Dostert01]

[DSLForum01]

[Dutta04]

$[\mathrm{EFM}]$

[ETSI97]

[Faulkner94]

[Faulkner04]

[Feldman97]

[Feldman98]

[Ferreira95]

[France04]
S.S. Dixit. IP over WDM, Building the Next Generation Optical Internet. John Wiley \& Sons, 2003, ISBN 0-471-21248-2.

K. Dostert. Powerline Communications, Prentice Hall, 2001, ISBN 0-13-029342-3.

Aspects of VDSL Evolution. DSL Forum Technical Report TR-040, June 2001. http://www.dslforum.org/aboutdsl/Technical'Reports/ TR-040.doc, [last access 25.12.2006].

A.K. Dutta, N.K. Dutta, M. Fujiwara. WDM Technologies, Optical Networks. Academic Press, 2004, ISBN 0-12-225263-2.

Ethernet in the First Mile Alliance. http://www.efmalliance.org/, [last access 28.07.2005].

ETS 300 681. Transmission and Multiplexing (TM); Optical Distribution Network (ODN) for Optical Access Network (OAN). ETSI Work Item DE/TM-01009, June 1997.

D.W. Faulkner, D. Gardan, L. Haagh, L. Larson, R. Margarito, M. Redstall, A. Socard, H. Steffensen, J. Stroetzel. Availability analysis of the fibre optic local loop. European Institute for Communications and Networks, 1994, pp. 28-32.

D.W. Faulkner, S. Fisher, P. Barker, P. Longhurst. Novel Fibre Infrastructure Solutions for Fibre-to-the-Premises in the UK. ECOC 2004 Proceedings, vol.5, pp. 22-25.

R.D. Feldman. Crosstalk and Loss in Wavelength Division Multiplexed Systems Employing Spectral Slicing. Journal of Ligthwave Technology, vol.15, no.11, November 1997, pp. 18231831.

R.D. Feldman, E.E. Harstead, S. Jiang, T.H. Wood, M. Zirngibl. An Evaluation of Architectures Incorporating Wavelength Division Multiplexing for Broad-Band Fiber Access. Journal of Lightwave Technology, vol.16, no.9, September 1998, pp. 1546-1558.

H.C. Ferreira, O. Hooijen. Power Line Communications: An Overview. The Transactions of the S.A. Institute of Electrical Engineers, September 1995, pp. 145-161.

P. France. Local Access Network Technologies. IEEE, 2004, ISBN 0-85296-176-6. 
[Frigo94]

[Frohberg01]

[FSAN]

[Gadonna94]

[Ghatak98]

[Gillespie01]

[Glance94]

[Gladisch04]

[Goff02]

[Golden08]

[Goodman05]

[Goralski01]

[Goure02]

[Green03]
N.J. Frigo, P.P Iannone, P.D. Magill, T.E. Darcie, M.M. Downs, B.N. Desai, U. Koren, T.L. Koch, C. Dragone, H.M. Presby, G.E. Bodeep. A Wavelength-Division Multiplexed Passive Optical Network with Cost-Shared Components. IEEE Photonics Technology Letters, vol.6, no.11, 1994, pp. 1365-1367.

W. Frohberg. Access-Technologien für den Zugang zu Telekommunikationsnetzen. Hüthig, 2001, ISBN 3-7785-3938-8.

Full Service Access Network. http://www.fsanweb.org/, [last access 21.12.2006].

M. Gadonna, M. Redstall, S. Gundersen. Reliability Evaluation for PON Power Splitters. Proceedings SPIE, The International Society for Optical Engineering, vol.2290, 1994, pp. 170-184.

A. Ghatak, K. Thyagarajan. Introduction to Fiber Optics. Cambridge University Press 1998, ISBN 0-521-57785-3.

A. Gillespie. Broadband Access Technology, Interfaces, and Management. Artech House, Inc., 2001, ISBN 0-89006-473-3.

B. Glance, I.P. Kamoniw, R.W. Wilson. Applications of the Integrated Waveguide Grating Router. Journal of Lightwave Technology, vol.12, No.6, June 1994, pp. 957-962.

A. Gladisch, S. Szuppa, R.P. Braun. 10Gb/s Ethernet In The Metro Area. ECOC 2004 Proceedings, Vol.3, pp. 390-392.

D.R. Goff. Fiber Optic Video Transmission: The Complete Guide. Focal Press, 2002, ISBN 0-240-80488-0.

P. Golden, H. Dedieu, K. S. Jacobsen. Implementation and Applications of DSL Technology. CRC Press, 2008, ISBN 0-84933423-3.

J.W. Goodman. Introduction to Fourier Optics. Roberts \& Company Publishers, 2005, ISBN 0-9747077-2-4.

W. Goralski. Optical Networking \& WDM. McGraw-Hill/Osborne 2001, ISBN 0-07-213078-4.

J.P. Goure, I. Verrier. Optical Fibre Devices. Institute of Physics Publishing, IOP, 2002, ISBN 0-7503-0811-7.

P.E. Green. Fiber-to-the-Home White Paper. Telecommunication Cosultant, Feb. 21, 2003. 
[Green06]

[Greenfield02]

[Gumaste02]

[Gumaste04]

[Haßlinger99]

[Hayashi04]

[Hecht02]

[Hecht04]

[Hecht05]

[Homeplug]

[Iannone96]

[Iannone98]

[Ims98]
P. E. Green. Fiber to the Home, The New Empowerment. WileyInterscience, 2005, ISBN 0-471-74247-3.

D. Greenfield. The Essential Guide to Optical Networks. Prentice Hall, 2002, ISBN 0-13-042956-2.

A. Gumaste, T. Antony. DWDM Network Designs and Engineering Solutions. Cisco Press, 2002, ISBN 1-58705-074-9.

A. Gumaste, T. Antony. First Mile Access Networks and Enabling Technologies. Cisco Press, 2004, ISBN 1-58705-129-x.

G. Haßlinger. Breitband-ISDN und ATM-Netze. Teubner Verlag 1999, ISBN 3-519-06251-8.

H. Hayashi, K. Ochiai, N. Sugimoto. $\mathrm{Bi}_{2} \mathrm{O}_{3}$-Based EDFAs with Cascaded Configurations for 4-Channel CWDM Systems. ECOC 2004 Proceedings, vol.3, pp. 512-513.

E. Hecht. Optics. $4^{\text {th }}$ edition, Addison Wesley, 2002, ISBN 0-32118878-0.

J. Hecht. City of Light, The Story of Fiber Optics. Oxford University Press, 2004, ISBN 0-19-516255-2.

J. Hecht. Understanding Fiber Optics. Prentice Hall, 2005, ISBN 013-117429-0.

Homeplug Powerline Industry Alliance. HomePlug 1.0 Specification. http://www.homeplug.com, [last access 27.12.2006].

P.P. Iannone, K.C. Reichmann, C.R. Doerr, M. Zirngibl, L. Iannone. Simultaneous WDM and Broadcast Transmission Using a Single Multiwavelength Waveguide-Grating-Router Laser. IEEE Photonics Technology Letters, vol.8, no.10, October 1996, pp. 13971399.

P.P. Iannone, K.C. Reicjmann, N.J. Frigo. High-Speed Point-toPoint and Multiple Broadcast Services Delivered over a WDM Passive Optical Network. IEEE Photonics Technology Letters, vol.10, no.9, September 1998, pp. 1328-1330.

L.A. IMS. Broadband Access Networks, Introduction strategies and techno-economic evaluation. Chapman \& Hall 1998, ISBN 0-41282820-0. 
[ITU-T G.7041] ITU-T Recommendation G.7041. Generic Framing Procedure (GFP), in Series G: Transmission Systems and Media, Digital Systems and Networks. Telecommunication Standardization Sector of ITU, December 2003.

[ITU-T G.982] ITU-T G.982. Optical access networks to support services up to the ISDN primary rate or equivalent bit rates. 11/1996.

[ITU-T G.983.1] ITU-T G.983.1. Broadband optical access systems based on Passive Optical Networks (PON). 11/1996.

[ITU-T G984.1] ITU-T G.984.1. General characteristics for Gigabit-capable Passive Optical Networks (GPON). 03/2003.

[ITU-T G.984.2] ITU-T G.984.2. Gigabit-capable passive optical networks (GPON): Physical media dependent (PMD) layer specification. 03/2003.

[ITU-T G.984.3] ITU-T G.984.3. Draft, Transmission Convergence Layer for Gigabit Passive Optical Networks. 03/2003.

[ITU-T G.992.1] ITU-T G.992.1. Asymmetric digital subscriber line (ADSL) transceivers. 03/2003.

[ITU-T G.992.2] ITU-T G.992.2. Splitterless asymmetric digital subscriber line (ADSL) transceivers. 03/2003.

[ITU-T G.992.3] ITU-T G.992.3. Asymmetric digital subscriber line transceivers 2 (ADSL2). 01/2005.

[ITU-T G.992.4] ITU-T G.992.4. Splitterless asymmetric digital subscriber line transceivers 2 (splitterless ADSL2). 07/2002.

[ITU-T G.992.5] ITU-T G.992.5. Asymmetric Digital Subscriber Line (ADSL) transceivers - Extended bandwidth ADSL2 (ADSL2+). 01/2005.

[James02] K.A. James, S.I. Fisher. Developments in optical access networks. BT Technology Journal, vol.20, no.4, October 2002, pp. 81-90.

[Jung01] D.K. Jung, H. Kim, K.H. Han, Y.C. Chung. Spectrum-sliced bidirectional passive optical network for simultaneous transmission of WDM and digital broadcast video signals. Electronics Letters, vol.37, no.5, 1st March 2001, pp. 308-309.

[Kaminow02a] I. Kaminov, T. Li. Optical Fiber Telecommunications IV A Components. Academic Press 2002, ISBN 0-12-395172-0. 
[Kaminow02b] I. Kaminov, T. Li. Optical Fiber Telecommunications IV B Systems and Impairments. Academic Press 2002, ISBN 0-12-395173-9.

[Kanbach99] A. Kanbach, A. Körber. ISDN - Die Technik, Schnittstellen Protokolle - Dienste - Endsysteme. Hüthig Verlag, 1999, ISBN 37785-2288-4.

[Kani03] J.I. Kani, M. Teshima, K. Akimoto, N. Takachio, H. Suzuki, K. Iwatsuki. A WDM-Based Optical Access Network for Wide-Area Gigabit Access Services. IEEE Optical Communications Magazine, vol.1, no.1, February 2003, pp. 43-48.

[Kartalopoulos00] S.V. Kartalopoulos. Introduction to DWDM Technology, Data in a Rainbow. IEEE Press, 2000, ISBN 0-7803-5399-4.

[Kashima93] N. Kashima. Optical Transmission for the Subscriber Loop. Artech House Publishers, Dezember 1993, ISBN 0-89006-679-5.

[Kashima95] N. Kashima. Passive Optical Components for Optical Fiber Transmission. Artech House Inc., 1995, ISBN 0-89006-775-9.

[Kauffels02] F.J. Kauffels. Optische Netze. Verlag mitp, 2002, ISBN 3-82664090-X.

[Keiser03] G. Keiser. Optical Communications Essentials. McGraw-Hill Professional, 2003, ISBN 0-07-141204-2.

[Kettler00] D. Kettler, H. Kafka, D. Spears. Driving Fiber to the Home. IEEE Communications Magazine, November 2000, pp. 106-110.

[Killat96] U. Killat. Access to B-ISDN via PONs, ATM Communication in Practice. John Wiley and Sons Ltd, 1996, ISBN 0-471-95877-8.

[Kitayama98] K. Kitayama. Code division multiplexing lightwave networks based upon optical code conversion. IEEE Journal on Selected Areas in Communications, vol.16, 1998, pp. 1309-1319.

[Klinger03] T. Klinger. Image Processing with LabVIEW and IMAQ Vision. Prentice Hall PTR, 2003, ISBN 978-0130474155.

[Kramer01] G. Kramer, B. Mukherjee, G. Pesavento. Ethernet PON (ePON): Design and Analysis of an Optical Access Network. Photonic Network Communications, vol.3, no.3, July 2001, pp. 307-319. 
[Kramer02] G. Kramer, B. Mukherjee, G. Pesavento. Interleaved Polling with Adaptive Cycle Time (IPACT): A Dynamic Bandwidth Distribution Scheme in an Optical Access Network. Photonic Network Communications, vol.4, no.1, 2002, pp. 89-107.

[Kramer03] G. Kramer, G. Pesavento. Ethernet Passive Optical Network (EPON): Building a Next-Generation Optical Access Network. IEEE Communications Magazine, February 2002, pp. 66-73.

[Kramer05] G. Kramer. Ethernet Passive Optical Networks. McGraw-Hill Professional Publishing, 2005, ISBN 0071445625.

[Krishna00] M.S. Krishna, S. Subramaniam. Optical WDM Networks Principles and Practice. Kluwer Academic Publishers, ISBN 0-7923-7825-3.

[Kyeong02] S.K. Kyeong. On the evolution of PON-based FTTH solutions. Information Sciences 149, 8 May 2002, pp. 21-30.

[Lin04] C.F. Lin. Optical Components for Communications. Kluwer Academic Publishers, 2004, ISBN 1402076363.

[Lin06] C. Lin. Broadband Optical Access Networks And Fiber-to-theHome, Systems Technologies and Development Strategies. John Wiley, 2006, ISBN 0-470-09478-8.

[Liu02]

K.H. Liu. IP over WDM. Wiley, 2002, ISBN 0-470-84417-5.

[Lund01]

B. Lund. Fiber-to-the-Home Network Architecture: A Comparison of PON and Point-to-Point Optical Access Networks. Proceedings of the National Fiber Optic Engineers Conference, 2001, pp. 15501557 .

[Maeda04] Y. Maeda. Overview of Optical Broadband in Japan. ECOC 2004 Proceedings, vol. 1, pp. 4-5.

[Mahlke01] G. Mahlke, P. Gössing. Fiber Optic Cables. Wiley Verlag, $4^{\text {th }}$ edition, 2001, ISBN 3-89578-162-2.

[Maier00] G. Maier, M. Martinelli, A.Pattavina, E.Salvadori. Design and Cost Performance of the Multistage WDM-PON Access Networks. Journal of Lightwave Technology, vol.18, 2. February 2000, pp. 125-143.

[Mauron01] P. Mauron. Reliability and lifetime of optical fibres and fibre Bragg gratings for metrology and telecommunications. Dissertation EPF no.2339, EMPA, 2001, pp. 77-78, ISBN 3-905594-24-2. 
[Mayhew02]

[McGarry06]

[Mestdagh95]

[Mickelsson02]

[Mickelsson03]

[Mickelsson04]

[Miller02]

[Milorad04]

[Mohammad03]

[Monroy02]

[Moon01]

[Mukherjee97]

[Nakanishi02]
A.J. Mayhew, S.J. Page, A.M. Walker, S.I. Fisher. Fibre to the home - infrastructure deployment issues. BT Technology Journal, vol.20, no.4, October 2002, pp. 91-103.

M.P. McGarry, M. Reisslein, M. Maier. WDM Ethernet Passive Optical Networks. IEEE Optical Communications, vol.44, no.2, February 2006, pp. S18-S25.

D.J.G. Mestdagh. Fundamentals of Multiaccess Optical Fiber Networks. Artech House Optoelectronics Library, 1995, ISBN 089006-666-3.

H. Mickelsson, E. Sundberg, P. Strömgren, Y. Fujimoto. IEEE Contribution on loop lengths. http://grouper.ieee.org/groups/802/3/efm/public/jan02/mickelsson '10102.pdf, IEEE EFM, January $14-16^{\text {th }}$, 2002, [last access $22.12 .2006]$.

H. Mickelsson, et al. Access Solution for a very Compact Point-toPoint FTTH Central Office Node. NFOEC Proceedings, 2003.

H. Mickelsson. Ethernet Based Point-to-Point Fibre Access Systems. ECOC 2004 Proceedings, vol.5, pp. 26-29.

J.L. Miller, E. Friedman. Optical Communications Rules of Thumb. McGraw-Hill, 2002, ISBN 0-07-138778-1.

C. Milorad. Optical Transmission Systems Engineering. Artech House, 2004, ISBN 1-58053-636-0.

I. Mohammad, T.M. Hussein. The Handbook of Optical Communication Networks. CRC Press, 2003, ISBN 0849313333.

I.T. Monroy, E. Tangdiongga. Crosstalk in WDM Communication Networks. Kluwer Academic Publishers, 2002, ISBN 1-4020-7026-8.

Y. Moon, C. Oh, K. Kim. MAC Protocols Supporting ITU-T Recommendation G.983.1 for Multimedia Services over ATM-Based PON. IEICE TRANS.COMMUN., vol.E84-B, no.2, February 2001, pp. 163-171.

B. Mukherjee. Optical Communication Networks. McGraw-Hill, 1997, ISBN 0-07-044435-8.

K. Nakanashi, Y. Maeda. Standardization Activities of FSAN: International Standardization Trends Concerning the Broadband PON (B-PON). NTT Review, vol.14, part2, 2002, pp. 108-110. 
[Oguchi96]

[Oksanen02]

[Olsen04]

[OPERA]

[Othonos99]

[Palais04]

[Parker98]

[Pavlidou03]

[Pedrotti07]

[Photeon05]

[Pike03]

[Pospischil95]
K. Oguchi. New Notations Based on the Wavelength Transfer Matrix for Functional Analysis of Wavelength Circuits and New WDM Networks Using AWG-Based Star Coupler with Asymmetric Characteristics. Journal of Lightwave Technology, vol.14. no.6, June 1996, pp. 1255-1263.

M. Oksanen, O.P. Hiironen, A. Tervonen, A. Pietiläinen, E. Gotsonoga, H. Järvinen, H. Kaaja, J. Aarnio, A. Gröhn, M. Karhiniemi, V. Moltchanov, M. Oikkonen, M. Tahkokorpi. Spectral Slicing Passive Optical Access Network Trial. Proceedings OFC 2002, Anaheim, pp. 439-440.

B.T. Olsen, K. Stordahl. Models for forecasting cost evolution of components and technologies. Telektronikk 4, 2004, pp. 138-145.

OPERA. Open PLC European Research Alliance. http://www.istopera.org, [last access 27.12.2006].

A. Othonos, K. Kalli. Fiber Bragg Gratings, Fundamentals and Applications in Telecommunications and Sensing. Artech House Publishers, 1999, ISBN 0-89006-344-3.

J.C. Palais. Fiber Optic Communications. Prentice Hall, Fifth Edition, 2004, ISBN 0-13-008510-3.

M.C. Parker, F. Farjady, S.D. Walker. Wavelength-Tolerant Optical Access Architectures Featuring N-Dimensional Addressing and Cascaded Arrayed Waveguide Gratings. Journal of Lightwave Technology, vol.16, no.12, December 1998, pp. 2296-2302.

N. Pavlidou, A.J. Han Vinck, J. Yazdani, B. Honary. Power Line Communications: State of the Art and Future Trends. IEEE Communications Magazine, April 2003, pp. 34-40.

F.L. Pedrotti, L.S. Pedrotti, L.M. Pedrotti. Introduction to Optics. Third Edition, Pearson, 2007, ISBN 0-13-149933-5.

Photeon technologies. Photeon Adds To Extensive Array of AWGs. Press Release, http://www.photeon.com/html/Press/2005 02 28.pdf, [last access 22.12.2006].

R. Pike, B. Neale. Corporate Finance and Investment, Decisions and Strategies. Prentice Hall, $4^{\text {th }}$ edition, 2003, ISBN 0-273-65138-2.

R. Pospischil. A bypass for the local loop: Deutsche Telekom's strategy for fiber to the home. Telecommunications Policy, vol.19, no.7, 1995, pp. 579-585. 
[Prat02]

[Que02]

[Ramamurthy01]

[Ramaswami02]

[Reed03]

[Sala01]

[Salvador99]

[Schiller03]

[Schussmann06]

[Schussmann07a]

[Schussmann07b]
J. Prat. Fiber-to-the-Home Technologies. Kluwer Academic Publishers. October 2002, ISBN 1-4020-7136-1.

Que. Special Edition Using Optical Networks. NIIT, 2002, ISBN 07897-2581-9.

B. Ramamurthy. Design of Optical WDM Networks, LAN, MAN and WAN Architectures. Kluwer Academic Publisher, 2001, ISBN 0-7923-7281-6.

R. Ramaswami, K.N. Kumar. Optical Networks, A Practical Perspective. Academic Press, 2002, ISBN 1-55860-655-6.

D. Reed. Copper Evolution. Federal Communications Commission, Technological Advisory Council III, Washington, DC, April 2003, http://www.fcc.gov/oet/tac/TAC ${ }^{\prime} I^{*} 04^{*} 17^{*} 03 /$ Copper Evolution.ppt, [last access 22.12.2006].

D. Sala, A. Gummalla. PON Functional Requirements: Services and Performance. Presented at IEEE802.3ah meeting in Portland, July 2001,http://grouper.ieee.org/groups/802/3/efm/public/jul01/presen tations/sala $1^{\circ} 0701 . p d f$ [last access 22.12 .2006$]$.

M.R. Salvador, S. Heemstra de Grot, I. Niemegeers. Protocol and networking design issues for local access WDM networks. Communication Protocols (COM) Group / Telematics Systems and Services (TSS) Centre for Telematics and Information Technology (CTIT) / University of Twente, February 1999.

J. Schiller. Mobilkommunikation. Pearson Studium, 2003, 2. Auflage, ISBN 3-8273-7060-4.

J. Schussmann, T. Schirl. Zugangsnetze der Zukunft werden Realität. Zeitschrift des Telematik Ingenieur Verbandes, TU Graz, 1/06, pp. 10-17.

J. Schussmann, T. Schirl. Design and Cost Performance of the WDM PON for Multi-Wavelength User. AccessNets 2007, August 22-24, Ottawa, Ontario, Canada.

J. Schussmann, T. Schirl. The Matrix Teaching Culture Quantifying Learning Output. Redesigning Pedagogy 2007, May 2830, Singapore. 
[Schussmann07c] J. Schussmann, T. Schirl. Design and Cost Performance of the WDM PON for Multi-Wavelength User. International Journal of Communication Networks and Distributed Systems (IJCNDS), ISSN 1754-3924, 2007.

[Schussmann08a] J. Schussmann, T. Schirl. Optische Zugangsnetze werden Realität. 2. Forschungsforum der österreichischen Fachhochschulen 2008, March 26-27, 2008.

[Schussmann08b] J. Schussmann, T. Schirl. Optical Access Networks and Advanced Photonics: Technologies and Deployment Strategies. Chapter: WDM PONs for Multi-Wavelength Users. A book edited by I.P. Chochliouros and A.G. Heliotis, IGI Global, 2008, submitted.

[Serrat03] J. Serrat, A. Galis. Deploying and Managing IP Over WDM Networks. Artech House Inc, 2003, ISBN 1-58053-501-1.

[Shannon48] C. E. Shannon. A Mathematical Theory of Communication. Bell System Technical Journal, vol. 27, 1948, pp. 379-423, 623-656.

[Shepard01]

[Siegmund97]

S. Shepard. Optical Networking Crash Course. McGraw-Hill Professional Publishing, 2001, ISBN 0-07-137208-3.

G. Siegmund. ATM - Die Technik, Grundlagen, Netze, Schnittstellen, Protokolle. Hüthig, 1997, 3. Auflage, ISBN 3-77852541-7.

[Simcoe02]

M. Simcoe. A Multiservice Voice, Data, and Video Network Enabled by Optical Ethernet. Proceedings of the National Fiber Optic Engineers Conference, 2002, pp. 119-129.

[Sivalingam05] K.M. Sivalingam, S. Subramaniam. Emerging Optical Networks Technologies. Springer, 2005, ISBN 0-387-22582-X.

[Smit96]

M.K. Smit, Cor van Dam. PHASAR-Based WDM-Devices: Principles, Design and Applications. IEEE Journal of Selected Topics in Quantum Electronics, vol.2, no.2, June 1996, pp. 236-250.

[Söderlund04] M. Söderlund, M. Kapulainen, J. Koponen. Techno-economic Comparison of Four Alternative FTTH Approaches in a Greenfield Building Site. ECOC 2004 Proceedings, vol.3, pp. 394-395.

[Son03]

E.S. Son, K.H. Han, J.K. Kim, Y.C. Chung. Bidirectional WDM Passive Optical Network for Simultaneous Transmission of Data and Digital Broadcast Video Service. Journal of Lightwave Technology, vol.21, No.8, August2003, pp. 1723-1727. 
[Spohn02]

[Stern88]

[Stern02]

[Stögner00]

[Suzuki04]

[Takahashi95]

$[\operatorname{Tan} 97]$

[Tanenbaum03]

[Tachikawa96]

[Tanis02]

[Tele99]
D.L. Spohn. Data Network Design. McGraw-Hill/Osborne, Third Edition, 2002, ISBN 0-07-219312-3.

J.R. Stern, C.E. Hoppitt, D.B. Payne, M.H. Reeve, K.A. Oakley. TPON - A passive optical network for telephony. Fourteenth European Conference on Optical Communication (ECOC88), vol.1, Brighton UK, September 1988, pp. 203-206.

T.E. Stern, K. Bala. Multiwavelength Optical Networks, A Layered Approach. Prentice Hall, 2002, ISBN 0-13-014711-7.

H. Stögner. Machbarkeitsstudie "Kärntner IntraNet" im Auftrag des Kärntner Wirtschaftsförderungsfonds (KWF). Interner Bericht, 2000 .

H. Suzuki, H. Nakamura, J. Kani, K. Iwatsuki. A wide area WDMbased passive optical network (WDM-PON) accommodating 10 Gigabit Ethernet-based VPN services. ECOC 2004 Proceedings, vol.2, pp. 272-273.

H. Takahashi, K. Oda, H. Toba, Y. Inoue. Transmission Characteristics of Arrayed Waveguide $\mathrm{NxN}$ Wavelength Multiplexer. Journal of Lightwave Technology, vol.13, no.3, March 1995, pp. 447-455.

A.H.H. Tan. SUPER PON - A Fiber to the Home Cable Network for CATV and POTS/ISDN/VOD as Economical as a Coaxial Cable Network. Journal of Lightwave Technology, vol.15, no.2, February 1997, pp. 213-218.

A.S. Tanenbaum. Computernetzwerke. 4. Auflage, Pearson Studium, 2003, ISBN 3-8273-7046-9.

Y. Tachikawa, K. Okamoto. Arrayed-waveguide grating lasers and their applications to tuning-free wavelength routing. IEE Proc.Optoelectronic, vol.143, no.5, October 1996, pp. 322-328.

D. Tanis, B.R. Eichenbaum. Cost of Coarse WDM Compared with Dense WDM for Wavelength-Addressable Enhanced PON Access. IEE Seminar on Photonic Access Technologies, December 2002.

O. Espvik. Broadband Access Networks. Telektronikk 2/3, vol.95, 1999, ISSN 0085-7130. 
[TERA]

[TONIC]

[UCSD05]

[Vetter04]

[Vic07]

[Vid07]

[Voges02]

[Volotinen99]

[Wagner89]

[Wan00]

[Wang04]
TERA. Techno Economic Results from ACTS. Project within the ACTS Programme from the European Commission, AC364, http://www.telenor.no/fou/prosjekter/tera/index.htm, 1994-2000, [last access 27.12.2006].

TONIC. Techno Economics of IP Optimised Networks and Services, Project within the IST Programme (Information Society Technologies) of the European Union, http://wwwnrc.nokia.com/tonic/, [last access 27.12.2006].

D. Ramsey. World's First International Real-time Streaming Of 4K Digital Cinema Over Gigabit IP Optical Fiber Networks. http://ucsdnews.ucsd.edu/newsrel/science/iGrid4K.asp, University of California, San Diego, 2005.

P.Vetter, J. Jaegher, M. Beck, K. Gustafsson, J. Wellen, M. Borgne, L. Humphrey, A. Foglar, R. Brink. Fibre Access in MUSE: an End-to-End Approach to Achieve Broadband for All. ECOC 2004 Proceedings, vol. 5, pp. 2-5.

Network Research Group, Lawrence Berkeley National Laboratory Accessible through the web at http://www.lbl.gov/LBL.html Network Research Group, "Vic - Video Conferencing Tool" Accessible through the web at http://www-nrg.ee.lbl.gov/vic/ [last access 30.03.2007].

VideoLAN, VideoLAN streaming solution, Accessible through the web at http://www.videolan.org/streaming, [last access 25.04.2007].

E. Voges, K. Petermann. Optische Kommunikationstechnik, Handbuch für Wissenschaft und Industrie. Springer-Verlag, Berlin, 2002, ISBN 3-540-67213-3.

T. Volotinen, W. Griffioen, M. Gadonna, H. Limberger. Reliability of Optical Fibres and Components, Final Report of COST 246. Springer, 1999, ISBN 185233147X.

S.S. Wagner, H.L. Lemberg. Technology and System Issues for a WDM-Based Fiber Loop Architecture. Journal of Lightwave Technology, vol.7, no.11, November 1989, pp. 1759-1768.

P.J. Wan. Multichannel Optical Networks. Kluwer Academic Publishers, 2000, ISBN0-7923-5776-0.

Z. Wang, X. Sun, C. Lin, C. Chan, L. Chen. A Novel Centrally Controlled Protection Architecture for Bidirectional WDM Passive Optical Network. ECOC 2004 Proceedings, vol. 2, pp. 168-169. 
[Webb00]

[Weldon03]

[Wellen04]

[Whitman04]

[Woesner98]

[Wolf00]

[Wosinska01]

[Yaojun99]

[Zheng01]

[Zheng03]

[Zirngibl95]
W. Webb. Introduction to Wireless Local Loop. Artech House Publishers, 2000, ISBN 1-58053-071-0.

M.K. Weldon, F. Zane. The Economics of Fiber to the Home Revisited. Bell Labs Technical Journal 8(1), 2003, pp. 181-206.

J.S. Wellen. Outlook: Next Generation FttX Access Networks. ECOC 2004 Proceedings, vol.5, pp. 30-33.

B. Whitman, M. Kunigonis. Fibre Access Deployment Worldwide Market Drivers. Politics and Technology Choices, ECOC 2004 Proceedings, vol.5, pp. 6-9.

H. Woesner. PrimeNet network design based on arrayed waveguide grating multiplexers. SPIE vol.3234, February 1998, pp. 22-28.

J. Wolf, N. Zee. The Last Mile. Broadband and the Next Internet Revolution. McGraw Hill, 2000, ISBN 0-07-136349-1.

L. Wosinska, L. Pedersen. Scalability Limitations of Optical Networks Due to Reliability Constraints. Proceedings of the National Fiber Optic Engineers Conference, 2001, pp. 1529-1540.

Q. Yaojun, Q.J. Pu Hongtu, C. Shuqiang, G. Kejian. A new scheme for WDM-Based passive optical access network. Beijing University of Posts and Telecommunications, 1999.

W. Zheng. Internet QoS Architectures and Mechanisms for Quality of Service. Morgan Kaufmann Publishers, 2001, ISBN 1558606084.

R. Zheng, D. Habibi. Emerging Architectures for Optical Broadband Access Networks. 2003.

M. Zirngibl, C.H. Joyner, L.W. Stulz, C. Dragone, H.M. Presby, I.P. Kaminow. LARnet, a Local Access Router Network. IEEE Photonics Technology Letters, vol.7, no.2, February 1995, pp. 215217. 


\section{Appendix A List of Abbreviations}

A

AAL

ADSL

AES

AND

AON

APON

ASON

ATM

AWG

AWGM

B

BBL

BER

B-ISDN

BPON

BWOUL

C

$\mathrm{CC}$

CD

CDM

CDMA

$\mathrm{CM}$

$\mathrm{CO}$

CPE

CPON

CSMA/CD

CWDM
ATM Adaptation Layer

Asymmetric Digital Subscriber Line

Advanced Encryption Standard

Access Network Designer

Active Optical Networks

ATM Passive Optical Network

Asymmetric Optical Access Network

Asynchronous Transfer Mode

Arrayed Waveguide Grating

Arrayed Waveguide Grating Multiplexer

Broadbandloop

Bit Error Rate

Broadband Integrated Services Data Network

Broadband Passive Optical Network

Broadcast Without Usual Limits

Constant Coarseness

Compact Disc

Code Division Multiplexing

Code Division Multiple Access

Cable Modem

Central Office

Customer Premise Equipment

Composite Passive Optical Network

Carrier Sense Multiple Access with Collision Detection

Coarse Wavelength Division Multiplexing 


\section{$\mathrm{D}$}

DBA

DC

DECT

DEMUX

DFB

DOCSIS

DSL

DVB-RCC

DWDM

\section{$\mathrm{E}$}

E1

EDGE

$\mathrm{EFM}$

$\mathrm{E} / \mathrm{O}$

EPON

ETSI

\section{F}

FBG

FCS

FDMA

FIT

FPI

FSAN

FSR

FTTB

FTTC

FTTcab

FTTD

FTTH

FTTO

FTTU

\section{G}

GB

$\mathrm{GbE}$

GBIC

GFP

GPON

GPRS
Dynamic Bandwidth Allocation

Decreased Coarseness

Digital Enhanced Cordless Telecommunications

Demultiplexer

Distributed Feedback

Data over Cable Service Interface Specification

Digital Subscriber Line

Digital Video Broadcasting Return Channel for Cable

Dense Wavelength Division Multiplexing

Europe 1, first multiplex level in Europes telephone systems

Enhanced Data Rates for Global Evolution

Ethernet in the First Mile

Electric/Optic

Ethernet Passive Optical Network

European Telecommunications Standards Institute

Fiber Bragg Grating

Frame Check Sum

Frequency Division Multiple Access

Failure in Time

Fabry-Perot Interferometer

Full Service Access Network

Free Spectral Range

Fiber to the Building

Fiber to the Curb

Fiber to the Cabinet

Fiber to the Desk

Fiber to the Home

Fiber to the Office

Fiber to the User

Grating Based

Gigabit Ethernet

Gigabit Interface Converter

Generic Framing Procedure

Gigabit Passive Optical Network

General Packet Radio Service 


\section{$\mathrm{H}$}

HDSL

High Data Rate Digital Subscriber Line

HDTV

High Definition Television

HFC

Hybrid Fiber Coaxial

HPON

Hybrid Passive Optical Network

HSCSD

High Speed Circuit Switched Data

HQHS

High Quality High Speed

\section{I}

IC

IFG

INT

IP

IPTV

IRR

ISDN

ISO

ISP

ITU

J

JPEG

Joint Photographic Experts Group

\section{K}

$\mathrm{KT}$

\section{L}

LAN Local Area Network

LARNet

LASER

LED

Local Access Router Network

Light Amplification by Stimulated Emission of Radiation

Light Emitting Diode

$\mathrm{M}$

MAC

MAN

MBS

MBWA

MMF

MON

Medium Access Control

Metropolitan Area Network

Multi Burst Slot

Mobile Broadband Wireless Access

Multi Mode Fiber

Multilevel Optical Network

MPCP 
MTBF

MTTR

MUSE

MUX

MZI

$\mathrm{N}$

NNI

NPV

NT

$\mathrm{O}$

OADM

OAM

OAMP

OAN

OCDM

ODN

$\mathrm{O} / \mathrm{E}$

OFDM

$\mathrm{OH}$

OLT

ONT

ONU

OPAL

OSI

OSNR

OTDM

$\mathrm{OXC}$

$\mathbf{P}$

P2MP

$\mathrm{P} 2 \mathrm{P}$

$\mathrm{P} 2 \mathrm{PE}$

PAL

$\mathrm{PC}$

PHASAR

PHY

PLC

PLL

PLOAM

PMD
Mean Time Before Failure

Mean Time To Repair

Multi-Service Access Everywhere

Multiplexer

Mach-Zehnder Interferometer

Network Node Interface

Net Present Value

Network Terminal/Termination

Optical Add Drop Multiplexer

Operation, Administration and Maintenance

Operation, Administration, Maintenance and Provisioning

Optical Access Network

Optical Code Division Multiplexing

Optical Distribution Network

Optic/Electric

Orthogonal Frequency Division Multiplex

Overhead

Optical Line Termination

Optical Network Termination

Optical Network Unit

Optisches Anschlussleitungssystem

Open System Interconnection

Optical Signal to Noise Ratio

Optical Time Division Multiplexing

Optical Cross Connect

Point to Multipoint

Point to Point

Point to Point Emulation

Phase Alternating Line

Personal Computer

Phased Array

Physical

Power Line Communication

Phase Locked Loop

Physical Layer Operation and Maintenance

Physical Medium Dependent 


$\begin{array}{ll}\text { PON } & \text { Passive Optical Network } \\ \text { POTS } & \text { Plain Old Telephone Service } \\ \text { PPP } & \text { Point-to-Point Protocol } \\ \text { PPS } & \text { Photonic Packet Switching } \\ \text { PSC } & \begin{array}{l}\text { Passive Star Coupler } \\ \text { PSPON }\end{array} \\ \text { PtP } & \text { Power-splitting PON to Point } \\ \text { Q } & \\ \text { QoS } & \text { Quality of Service } \\ \text { R } & \\ \text { RF } & \text { Radio Frequency } \\ \text { RITENET } & \text { Remote Interrogation of Terminal Network } \\ \text { RoCS } & \text { Reconvergence of Communication Services }\end{array}$

S

\begin{tabular}{ll} 
SAN & Storage Area Network \\
SDH & Synchronous Digital Hierarchy \\
SDK & Software Development Kit \\
SDM & Space Division Multiplexing \\
SDSL & Symmetric Digital Subscriber Line \\
SEC & Section \\
SHD & Super High Definition \\
SLA & Service Level Agreement \\
SME & Shared Medium Emulation \\
SMF & Single Mode Fiber \\
SNR & Signal to Noise Ratio \\
SONET & Synchronous Optical Network \\
SP & Service Provider \\
ST & Stage \\
& \\
T & \\
T1 & Trunk 1, first multiplex level in US American telephone systems \\
TC & Transmission Convergence Layer \\
TDM & Time Division Multiplexing \\
TDMA & Time Division Multiple Access \\
TERA & Techno Economic Results from ACTS \\
TETRA & Terrestrial Trunked Radio System \\
TFF & Thin Film Filter \\
THX & Thanks \\
TV & Television \\
\hline
\end{tabular}




\section{$\mathrm{U}$}

UMTS

UNE

UNI

USB

UTOPIA

\section{V}

V.x

VBR

$\mathrm{VC}$

VDSL

VoD

VoIP

VPN

\section{W}

WAN

WDM

WGR

$\mathrm{WiFi}$

WiMAX

WLAN

WMAN

WPAN

WR

WTM

WWDM

WWW

\section{$\mathrm{X}$}

$\mathrm{xDSL}$
Universal Mobile Telecommunications System

User Network Equipment

User Network Interface

Universal Serial Bus

Utah Telecommunications Open Infrastructure Agency

Standardized transmission methods for telephone modems from ITU Variable Bit Rate

Virtual Channel

Very High Data Rate Digital Subscriber Line

Video on Demand

Voice over Internet Protocol

Virtual Private Network

Wide Area Network

Wavelength Division Multiplexing

Wavelength Grating Router

Wireless Fidelity

Worldwide Interoperability for Microwave Access

Wireless Local Area Network

Wireless Metropolitan Area Network

Wireless Personal Area Network

Wavelength Router

Wavelength Transfer Matrix

Wide Wavelength Division Multiplexing

World-Wide Web

Different types of DSL 


\section{Appendix B List of used Symbols}

\subsection{List of General Symbols}

$\begin{array}{ll}\text { e.g. } & \text { for example (Lat. exempli gratia) } \\ \text { et al. } & \text { and others (Lat. et alii) } \\ \text { etc. } & \text { et cetera } \\ \text { et seq. } & \text { following pages (Lat. et sequitur) } \\ \text { i.e. } & \text { that is (Lat. id est) } \\ \text { no. } & \text { number } \\ \text { p. } & \text { page } \\ \text { pp. } & \text { pages } \\ \text { para. } & \text { paragraph } \\ \text { pt. } & \text { part } \\ \text { q.v. } & \text { which see (Lat. quod vide) } \\ \text { sec. } & \text { section } \\ \text { vol. } & \text { volume }\end{array}$

Physical units are written in squared brackets. References to further literature or citations are also written in squared brackets. Footnotes are denoted by superscripted numbers, for example $\mathrm{WDM}^{2}$.

Equations, cross references, tables, figures and page numbers are listed in parentheses. Equations are numbered in ascending order (e.g. (3.24)). Cross references are performed by the number of the chapter, section, subsection, paragraph or by the page number. Tables and figures are listed by the term "Table:" and "Figure:" respectively, followed by the corresponding number.

Other references and additional information which refer to the appendix are marked with a capital letter, followed by the adequate number (e.g. C1).

For references to further literature, the abbreviation "p." relates to the listed page, however, the abbreviation "pp." refers to the specified pages. For example, a reference ([Gumaste04], p. 30 et seq.), means a reference to the literature [Gumaste04], to the page 30 and the following pages. References to literature with a team of more than three authors are abbreviated with the abbreviation et al. 


\subsection{List of Mathematical Symbols}

\section{Greek Symbols}

$\alpha_{c}$

$\alpha_{\mathrm{f}}$

$\alpha_{i}$

$\alpha_{\text {imax }}$

$\alpha_{\mathrm{m}}$

$\alpha_{\text {round-trip }}$

$\alpha_{\mathrm{s}}$

$\alpha_{\text {smax }}$

$\alpha_{\text {tmax }}$

$\alpha_{\text {tmin }}$

$\beta$

$\delta$

$\varepsilon$

$\varepsilon_{0}$

$\varepsilon_{\mathrm{r}}$

$\Delta \Phi$

$\Delta \mathrm{l}$

$\phi^{\mathrm{ik}}$

$\eta$

$\eta_{\text {var }}$

$\eta_{\text {const }}$

$\eta_{\text {phys }}$

$\kappa$

$\Lambda^{\mathrm{k}}$

$\Delta \lambda$

$\Delta \lambda_{\text {tr }}$

$\lambda$

$\lambda_{0}$

$\lambda_{\mathrm{a}}^{\mathrm{b}}$

$\mu$

$\mu_{0}$

$\mu_{\mathrm{r}}$

$\sigma_{\mathrm{i}}$

$\tau_{\text {tr }}$

$\tau_{\text {rec }}$

$\omega$
Connector loss $[\mathrm{dB}]$

Fiber loss $[\mathrm{dB} / \mathrm{km}]$

Insertion loss $[\mathrm{dB}]$

Maximum insertion loss $[\mathrm{dB}]$

System margin $[\mathrm{dB}]$

Round-trip loss $[\mathrm{dB}]$

Splitting loss $[\mathrm{dB}]$

Maximum splitting loss $[\mathrm{dB}]$

Maximum total loss $[\mathrm{dB}]$

Minimum total loss [dB]

Propagation constant

Kronecker delta

Dielectricity

Dielectric constant in vacuum: $8.854187817 \cdot 10^{-12} \mathrm{Fm}^{-1}$

Dielectric coefficient

Phase difference

Length difference

Relative phases between $\mathrm{i}$ and $\mathrm{k}$

Efficiency

Efficiency for variable frame or cell size

Efficiency for constant frame or cell size

Efficiency of the physical bit rate

Coupler transmission factor

Elements of a WTM

Channel spacing

Spectral broadness of the transmitter

Wavelength

Base wavelength

Wavelength; subscripted character represents the discrete wavelength number; superscripted character represents the port number

Permeability

Permeability constant in vacuum: $4 \pi \cdot 10^{-7} \mathrm{Vs} / \mathrm{Am}$

Permeability coefficient

Standard deviation loss of component i

Rise time of the transmitter

Rise time of the receiver

Angular frequency $\left[\mathrm{s}^{-1}\right]$ 


\section{Latin Symbols}

a

B

$\mathrm{B}_{0}$

$\mathrm{B}_{\mathrm{N}}$

$\mathrm{C}$

$\mathrm{C}_{\mathrm{s}}$

$\overrightarrow{\mathrm{c}}$

C

$\mathrm{C}_{\mathrm{f}}$

$\mathrm{C}_{\mathrm{c}}$

D

$\mathrm{D}_{\max }$

d

$\mathrm{d}^{\mathrm{ij}}$

$\Delta \mathrm{F}$

$\Delta \mathrm{f}$

$\mathrm{f}$

fps

FSR

\#FSR

$\mathrm{g}$

$\mathrm{h}$

$\mathrm{IN}_{\mathrm{s}}$

$\mathrm{INT}_{\mathrm{s}}$

$\mathrm{INT}_{\mathrm{s}}^{-1}$

i

$\tilde{\mathrm{i}}$

I

$\overrightarrow{\mathrm{I}}$

$\mathrm{I}_{\mathrm{m}}$

$\mathrm{k}$

$\Delta \mathrm{l}$

L

$\mathrm{L}_{\mathrm{i}}$

1

M

$\overrightarrow{\mathrm{M}}$

Move $_{n}$

$\mathrm{m}$

N

$\overrightarrow{\mathrm{N}}$

$\mathrm{N}_{\omega}$

NET

$\mathrm{NET}^{-1}$

$\mathrm{n}$

$\mathrm{n}_{\omega}$
Interval between terminated input ports of a WR

Bandwidth

Channel bitrate [bit/s]

Network capacity limit [bit/s]

Coarseness factor

Coarseness factor in stage s

Vector containing the coarseness factor

Cost $[€]$

Fiber cost $[€ / \mathrm{m}]$

Channel capacity [bit/s]

Distance between OLT and ONU $[\mathrm{m}]$

Maximum distance between OLT and ONU [m]

Distance $[\mathrm{m}]$

Difference in distance between $i$ and $j$

Accessible optical bandwidth

Channel spacing

Frequency $\left[\mathrm{s}^{-1}\right]$

Frames per seconds

Free spectral range

Number of free spectral range

Group of adjacent wavelengths

Planck's constant: $6.6260693 \cdot 10^{-34} \mathrm{Js}$

Input matrix in stage $\mathrm{s}$

Interstage operator in stage $\mathrm{s}$

Inverse interstage operator in stage $s$

Input port number

Generic terminated input port

Number of input ports

Vector containing the number of input ports used

Input matrix of the size $\mathrm{m}$

Number of the discrete wavelength

Length difference

Frame or cell size

Mean loss of component i

Length

Size of the WR

Vector containing the size of the WR

Move operator for $\mathrm{n}$ times shifting

Number of arrayed waveguides

Size of the passive star coupler or WR

Vector containing the number of WR

Total number of wavelengths in the whole network

Network operator

Inverse Network operator

Number of inputs and outputs

Number of wavelengths per fiber 


$\begin{array}{ll}\overrightarrow{\mathrm{n}}_{\omega} & \text { Vector containing the number of wavelengths per fiber } \\ \mathrm{n}_{\mathrm{p}} & \text { Receiver sensitivity [photon/bit] } \\ \mathrm{n}_{\mathrm{x}} & \text { Refractive index } \\ \mathrm{O} & \text { Output port number } \\ \mathrm{O} & \text { Number of output ports } \\ \mathrm{OH} & \text { Overhead [Byte] } \\ \mathrm{O}_{\mathrm{m}} & \text { Output matrix of the size } \mathrm{m} \\ \mathrm{OUT}_{\mathrm{s}} & \text { Output matrix in stage s } \\ \mathrm{p} & \text { Number of possibilities } \\ \mathrm{P}_{\mathrm{in}} & \text { Received optical power } \\ \mathrm{P}_{\text {out }} & \text { Optical power at the sender } \\ \mathrm{P}_{\mathrm{R}} & \text { Receiver sensitivity } \\ \mathrm{P}_{\mathrm{S}} & \text { Optical power at the sender } \\ \mathrm{R} & \text { Radius of a circle } \\ \mathrm{S} & \text { Number of stages } \\ \mathrm{S} / \mathrm{N} & \text { Signal to noise ratio } \\ \mathrm{ST}_{\mathrm{s}} & \text { Stage operator in stage s } \\ \mathrm{ST}_{\mathrm{s}}^{-1} & \text { Inverse stage operator in stage s } \\ \mathrm{s} & \text { Stage number } \\ \mathrm{t} & \text { Time } \\ \mathrm{T} & \text { Transfer function } \\ \mathrm{U} & \text { Number of users } \\ \mathrm{U}_{\omega} & \text { Number of wavelengths per user } \\ \mathrm{v} & \text { Propagation speed } \\ \left.\mathrm{V}_{\mathrm{n}}{ }_{\mathrm{n}} \mathrm{k}\right) & \text { Number of variations with repetition } \\ \mathrm{WTM} & \text { Wavelength transfer matrix } \\ \mathrm{x}_{\text {res }} & \text { Horizontal resolution } \\ \mathrm{X} & \text { Payload [Byte] } \\ \mathrm{y}_{\text {res }} & \text { Vertical resolution } \\ \mathrm{z} & \text { Index inside a consecutive wavelength comb } \\ & \end{array}$

\section{Other Symbols}

$*$

${ }^{\circ} \mathrm{C}$

$\#$

L」
Modified matrix multiplication

Degree celsius

Number of

Integer part 


\section{Appendix C Standards}

This chapter itemize the most important standards related to optical access networks based on TDM and WDM.

ITU-T G.691 Optical interfaces for single channel STM-64, STM-256 systems and other SDH systems with optical amplifiers. This document was approved in 2000 and specifies the optical physical layer of the SDH system.

ITU-T G.692

Optical interfaces for multichannel systems with optical amplifiers. It was approved in 1998.

ITU-T G.693

ITU-T G.694.1

ITU-T G.694.2

Optical interfaces for intra-office applications. Adopted in 2001, this recommendation enables transverse (multi-vendor) compatibility.

Spectral grids for WDM applications: DWDM frequency grid. It was approved in 2002 .

Spectral grids for WDM applications: CWDM frequency grid. It was adopted in 2002 .

ITU-T G.957

Optical interfaces for equipments and systems relating to the synchronous digital hierarchy. This document was approved in 1999.

ITU-T G.959.1 Optical transport network physical layer interfaces. This recommendation was ratified in 2001.

ITU-T G.982

Optical access networks to support services up to the ISDN primary rate or equivalent bit rates. This document was approved in 1996.

ITU-T G.983.1 Broadband optical access systems based on Passive Optical Networks (PON). This document was approved in 1998 and specifies the optical physical layer of the APON/BPON system. 
ITU-T G.983.2

ITU-T G.983.3

ITU-T G.983.4

ITU-T G.983.5

ITU-T G.983.6

ITU-T G.983.7

ITU-T G.983.8

ITU-T G.984.1

ITU-T G.984.2

ITU-T G.984.3
ONT management and control interface specification for B-PON. Adopted in 2002, this recommendation defines a common management control interface.

A broadband optical access system with increased service capability by wavelength allocation. Specification for wavelength overlay to support additional services such as analog video. This recommendation was ratified in 2001.

A broadband optical access system with increased service capability using dynamic bandwidth assignment. Describes necessary mechanism to support dynamic bandwidth allocation among multiple ONT's in the same PON.

A broadband optical access system with enhanced survivability. Adopted in 2002, this document specify the access line protection for BPON.

ONT management and control interface specifications for B-PON system with protection features. Adopted in 2002, this document defines the extensions for control interface for management of protection switching functions at the ONT.

ONT management and control interface specification for dynamic bandwidth assignment (DBA) B-PON system. This document defines the extensions for control interface necessary for management of DBA functions at the ONT. It was approved in 2001.

B-PON OMCI support for IP, ISDN, Video, VLAN Tagging, VC Cross-Connections and other select functions. Adopted in 2003, this recommendation specifies the extensions for control interface necessary for management of various extended services at the ONT.

General characteristics for Gigabit-capable Passive Optical Networks (GPON). This document was approved in 2003 and specifies the optical physical layer of the GPON system.

Gigabit-capable passive optical networks (GPON): Physical media dependent (PMD) layer specification. This document was approved in 2003 and specifies the optical physical layer of the GPON system.

Transmission Convergence Layer for Gigabit Passive Optical Networks. It was approved in 2003. 
ITU-T G.985

IEEE802.3ah
$100 \mathrm{Mbit} / \mathrm{s}$ point-to-point Ethernet based optical access system. This document was approved in 2003 and specifies the optical physical layer of $100 \mathrm{Mbit} / \mathrm{s}$ point-to-point Ethernet system.

Ethernet in the First Mile [EFM]. 


\section{Index}

3

3-D Stage Operator 94

3-dB Coupler 18, 22, 23, 29

\section{A}

Active Architectures 16

APON 31

Architecture Index 116

Architectures Possible 122

Arrayed Waveguide Grating 37

ATM 32, 34

AWGM 37,39

\section{B}

Base Wavelength 55

Bidirectional Ring 61

Bitrate Limitation 8

Bitrate Requirements 6

Bottom-Up Approach 98

BPON 31

Broadcast-and-Select Networks 17

Burst Switching 19

Bus 18, 29

\section{C}

Cable Cost 115, 117

Capacity Limit 25, 72, 104

Coarse Wavelength Division Multiplexing 18

Coarseness 56, 63, 65, 70, 76, 84, 85, 93, 111

Code Division Multiple Access 19, 20
Combiner 22

Common Design Parameter 79

Computation Complexity 97, 100

Connectivity Map 70, 91, 101, 102

Constraints 78

Coraseness 65

Cost Evaluation 115

Cost Evolution 114

Cost Function 114

Cost per User 121

Cost Performance 114

Coupler 22

Coupler Losses 23

Coupler Transmission Factor 23

CWDM 47

Cyclic Routing Property 67, 81, 99

Cycling Routing Property 56

\section{D}

DC WDM PON 77, 85, 88, 102, 109, 110

Design Conventions 75, 79

Digital Subscriber Line 11

Discrete Bidimensional Domain 54

Downstream Transmission 42

DWDM 42,47

\section{$\mathrm{E}$}

EPON 31, 33

Ethernet 21, 33

Ethernet Passive Optical Network 21 


\section{F}

free Spectral Range 54

Free Spectral Range 41, 63, 65, 66, 67, 70,85

Full Service Access Network 31

G

Generation of Architectures 110

GPON 31, 32

Group of Adjacent Channels 81

\section{$\mathrm{H}$}

Hybrid Fiber Coaxial 11

\section{I}

IC WDM PON 77, 81, 88, 89, 102, 109, 110

Identity Matrix 93

Input Matrix 92, 98

Installation Cost 115, 117

Interstage Operator 92

Inverse 3-D Stage Operator 98

Inverse Discrete Transfer Function 100

Inverse Interstage Operator 98

Inverse NET Operator 100

Inverse Space Switching Function 98

Inverse Stage Operator 98

\section{$\mathrm{K}$}

Kronecker Delta 41

\section{$\mathrm{L}$}

LARNet 45

Link Length 106

Link Lengths 115

Little Gauss 122

\section{M}

Maximum Number of Architectures 112

Metropolitan Area Networks 18

Minimum Cost Architectures 114, 119

Modified Matrix Multiplication 64, 67

Move Operator 95, 99

Multi-hop Networks 17

Multistage WDM PON 74, 78, 106, 120,136
MUSE 52

\section{$\mathrm{N}$}

NET Operator 96, 97

Network Architecture 120

Network Capacity 79

Network Design 67, 75

Network Model 78

Number of Architectures 111

Number of Required WR 80

Number of Wavelengths per User 88

\section{O}

OADM 39

Optical Access Networks 15

Optical Loopback 44

Optical Spectrum Access 25

Optical Time Division Multiplexing 19

Optisches Anschlussleitungssystem 16

Output Matrix 92, 98

Overhead Impact 34

$\mathbf{P}$

Paradigm Shift 49

Passive Architectures 16

Passive Star Coupler 18

Performance Analysis 115, 123

Phase Difference 40

Photonic 3

Photonic Packet Switching 19

Point-to-Point 21

Position Index 123, 124

Power Budget 106

Power Budget Limit 25

Power-line Communication 12

Propagation Constant 40

R

Receiver Sensitivity 27, 106

Required Number of Input Ports 80

Ring 18

RITENET 44

Routing Function 56

Rowland Circle 41 


\section{$\mathrm{S}$}

Single-hop Networks 17

Single-Stage WDM PON 54, 59

Space Demultiplexing 54, 57, 82

Space Division Multiplexing 19, 21

Spectral Grid 47

Spectral Slicing 45

Splitter 22

Stage Operator 92

Standardization 47

Standards 165

Star 18

Star Coupler 24

Symmetry 7

\section{$\mathrm{T}$}

\section{TDM PON 22, 31}

Techno- Economic Evaluation 108

Temperature Fluctuations 89

Test Environment 127, 137

Three Dimensional Matrix 93

Time Division Multiplexing 19

Top-Down Approach 92

Total Number of Used Wavelengths 79

Transmission time 8, 10

Tunable Source 43

\section{$\mathrm{U}$}

Upstream Transmission 43

UTOPIA 52

\section{$\mathrm{V}$}

Variations with Repetitions 110

\section{W}

Wavelength Division Multiplexing 19

Wavelength Interval 55, 82

Wavelength Reuse 54, 82

Wavelength Transfer Matrix 59, 65

Wavelength-Routing 18

Wavelength-Routing Networks 17

Wavelength-Switching Networks 17

WDM 3

WDM PON 37, 54, 57, 67, 74, 88, 91, 99

Wide Area Networks 18

WR 37, 67, 78, 81, 110

WR Cost 114, 116

WR Position 120, 123, 124, 126

WTM Generalization 63

WWDM 47 